\title{
Graphical Passwords and Practical Password Management
}

By

\author{
Elizabeth Ann Stobert
}

A thesis submitted to

the Faculty of Graduate and Postdoctoral Affairs

in partial fulfilment of

the requirements for the degree of

\section{DOCTOR OF PHILOSOPHY}

in

Computer Science

Carleton University

Ottawa, Ontario, Canada

(c)2015, Elizabeth Ann Stobert 


\section{Abstract}

Text passwords pose a number of difficulties for end users, who must create, remember, and manage large numbers of passwords. Users are often regarded as the weak link in security systems, but they are a crucial component of the system, and need to be better considered in the design of security products. Many password alternatives have been proposed, but none have successfully replaced ordinary text passwords, and the potential consequences of password problems grow as more information relating to work and life is stored online.

This thesis explores practical approaches to helping users select, securely reuse, and manage passwords, and investigates questions about password alternatives. The attention is on the end user, and how authentication affects these users in their daily lives. Our focus is on practical, actionable results to assist end users in their daily tasks.

The thesis begins by investigating issues of memorability with graphical passwords, and proposes the design of PassTiles, a new graphical password system that allows secure random memorable passwords to be easily assigned. This graphical password system is used to explore what type of memory retrieval best supports the memorability of graphical passwords, and the results show that cued-recall graphical passwords give an advantageous combination of memorability and usability.

Password coping strategies are next explored through interviews with end users, and investigation into the techniques that users rely on to handle current password demands. Interviews with expert users were conducted to understand how their additional expertise helps them manage the same problems faced by end users. Grounded Theory analysis led to the emergence of a password life cycle model. A survey study suggested that the coping strategies discussed in the interviews are widespread.

Finally, the thesis proposes the design of a password manager to support users' existing coping strategies by protecting password reuse, and to securely protect users' accounts with memorable assigned random graphical passwords. 


\section{Acknowledgements}

I have had a lot of fun working on this PhD. While not without moments of challenge and frustration, this has been a hugely enjoyable and fulfilling experience, and I owe much to the people who have supported me throughout it.

I can't begin to list what I've learned from my supervisor, Robert Biddle. Robert's enthusiasm and broad knowledge are an inspiration and made working with him a pleasure. Thank you for showing me that I could combine all of my areas of interest, and believing that I could be good at this. I would also like to thank my mentor Sonia Chiasson, for her advice, friendship, career inspiration, and not least for answering all of my questions.

My friends and family have provided me with so much love and support throughout my time as a student. I'd like to thank my parents for their unwavering enthusiasm, and especially thank my mum for sharing her statistics expertise and being a fantastic role model. I'd also like to thank my friend Emily Miller-Cushon for her discussion, commiseration, and amazing advice. And I am incredibly grateful to my husband David for all of his love, patience, proof-reading, and hand-holding.

I would like to give many thanks to my Carleton University colleagues in the HotSoft, CHORUS, and CCSL research groups. Their feedback on paper drafts and practice presentations, and their willingness to lend their help and expertise to my studies has improved my research. Particularly, I'd like to thank Alain Forget for his help in running studies. I would also like to thank Srđan Čapkun and the Systems Security research group at ETH Zürich for having welcomed me as a visiting researcher. They provided me with the support and academic environment that I needed to complete this thesis when I was a long way from my own research group.

Finally, I must thank all of the participants who took part in my studies. This research would have been impossible without their generous contribution of time and effort. 


\section{Contents}

$\begin{array}{ll}\text { Abstract } & \text { ii }\end{array}$

Acknowledgements

Table of Contents $\quad$ iv

List of Tables $\quad$ ix

List of Figures $\quad$ xi

1 Introduction 1

1.1 Research Problem . . . . . . . . . . . . . . . . . . . . 1

1.2 Contributions . . . . . . . . . . . . . . . . 3

1.3 Organization of Thesis . . . . . . . . . . . . . . . 4

1.4 Related Publications . . . . . . . . . . . . . . . . 5

2 Background and Related Work $\quad 8$

2.1 Authentication . . . . . . . . . . . . . . . . 8

2.1.1 Password Security . . . . . . . . . . . . . . . . . . . . . 9

2.2 The Password Problem . . . . . . . . . . . . . . . . . . . . . . . . . . 11

2.3 Users and Passwords . . . . . . . . . . . . . . . . . . . . . . 13

2.3.1 Number of Passwords . . . . . . . . . . . . . . . . . . . . 13

2.3 .2 Password Choice . . . . . . . . . . . . . . . . . . 15

2.3 .3 Password Reuse . . . . . . . . . . . . . . . . . . . . . . . 18

2.3 .4 Password Recording . . . . . . . . . . . . . . . . 20

2.3.5 Password Sharing . . . . . . . . . . . . . . . . . . . . 21

2.4 Addressing the Password Problem . . . . . . . . . . . . . . . 22

2.4.1 Alternative Text Password Schemes . . . . . . . . . . . . 23

2.4.2 Graphical Passwords . . . . . . . . . . . . . . . . . . 26

2.4 .3 Password Managers . . . . . . . . . . . . . . . . . . . 31 
2.4.4 Single Sign-on . . . . . . . . . . . . . . . . . . . . . 34

2.4.5 Challenge Questions . . . . . . . . . . . . . . . . 34

2.5 Memory Theory for Passwords . . . . . . . . . . . . . . . 35

2.5.1 Picture Superiority Effect . . . . . . . . . . . . 35

2.5.2 Memory Retrieval . . . . . . . . . . . . . . . . . 36

2.5.3 Memory Retrieval and Graphical Passwords . . . . . . . . . 38

2.6 Summary . . . . . . . . . . . . . . . . . . . . . . . . 39

3 PassTiles 41

3.1 Introduction . . . . . . . . . . . . . . . . . . 41

3.2 Original PassTiles . . . . . . . . . . . . . . . . . . . . . . . . . 43

3.3 Further Modifications . . . . . . . . . . . . . . . . . . . . . . 44

3.4 Usability . . . . . . . . . . . . . . . . . . . . . . . . . . . . . . . 48

3.5 Security . . . . . . . . . . . . . . . . . . . . 49

3.5 .1 Guessing Attacks . . . . . . . . . . . . . . 50

3.5 .2 Capture Attacks . . . . . . . . . . . . . . . . 52

3.6 Conclusion . . . . . . . . . . . . . . . . . . . . . . . . 53

4 Memory Retrieval and Graphical Passwords $\quad 54$

4.1 Introduction . . . . . . . . . . . . . . . . . . . . . 54

4.2 Study Design . . . . . . . . . . . . . . . . . . . 55

4.3 Password Results . . . . . . . . . . . . . . . . 60

4.3 .1 Studies 1 and $2 \ldots \ldots \ldots \ldots \ldots \ldots$

$4.3 .2 \quad$ Follow-up Studies . . . . . . . . . . . . . . . . . . . 63

$4.3 .3 \quad$ Study $3 \ldots \ldots \ldots \ldots \ldots$

$4.3 .4 \quad$ Study $4 \ldots \ldots \ldots \ldots$

$4.3 .5 \quad$ Study $5 \ldots \ldots \ldots \ldots \ldots \ldots \ldots \ldots$

4.4 Discussion . . . . . . . . . . . . . . . . . . . . . . 76

4.5 Conclusion . . . . . . . . . . . . . . . . . . . . 79

5 The Password Life Cycle $\quad 81$

5.1 Introduction . . . . . . . . . . . . . . . . . 81

$5.2 \quad$ Study . . . . . . . . . . . . . . . . . . . . . . . . . . . . . . 82

5.3 Results Overview . . . . . . . . . . . . . . . . . . . . . . . 84

5.4 Qualitative Analysis . . . . . . . . . . . . . . . . . . 87

5.4 .1 Open Coding . . . . . . . . . . . . . . . . . . . 87

$5.4 .2 \quad$ Axial Coding . . . . . . . . . . . . . . . . . . . . . . . 89

5.4 .3 Selective Coding . . . . . . . . . . . . . . . . . 103

5.4.4 The Password Life Cycle . . . . . . . . . . . . . . . . . 107

$5.5 \quad$ Life Cycle Example . . . . . . . . . . . . . . . . . . . . . . . . . . 109

5.6 Discussion . . . . . . . . . . . . . . . . . . . . . . . . . . . . . 111

5.6 .1 Writing Passwords Down . . . . . . . . . . . . . 111

5.6 .2 Password Cues . . . . . . . . . . . . . . . . . . . . 112 
5.6 .3 Password Managers . . . . . . . . . . . . . . . . . . . . 112

5.6 .4 Single Sign-On . . . . . . . . . . . . . . . . . . . . 113

5.6 .5 Extra Information . . . . . . . . . . . . . . . . . 114

5.6 .6 Threat models . . . . . . . . . . . . . . . . . . . . 115

5.7 Conclusion . . . . . . . . . . . . . . . . . . . 116

6 Expert Password Management 117

6.1 Introduction . . . . . . . . . . . . . . . . . . . . 117

6.2 Study . . . . . . . . . . . . . . . . . . . . . . . . . . . . . . . 118

6.3 Results . . . . . . . . . . . . . . . . . . . . . . . . . . . . 119

6.3 .1 Results Overview . . . . . . . . . . . . . . . . . . . . . . . 119

6.3.2 Qualitative Observations . . . . . . . . . . . . . . 120

6.4 Discussion . . . . . . . . . . . . . . . . . . . . . . . . . . 124

6.4.1 Comparing to Non-Experts _ . . . . . . . . . . . . 125

6.4.2 What Do Experts Do Right? . . . . . . . . . . . . . 126

6.4.3 Remaining Problems with Password Management . . . . . . 127

6.5 Conclusion . . . . . . . . . . . . . . . . . . . . 128

7 Surveying Users about Password Management 130

7.1 Introduction . . . . . . . . . . . . . . . . . . . . . . . . . 130

$7.2 \quad$ Study . . . . . . . . . . . . . . . . . . . . . . . . . 131

7.2 .1 Pilot Testing . . . . . . . . . . . . . . . . . . . . . 132

$7.2 .2 \quad$ Survey Participants . . . . . . . . . . . . . . . . . . 133

7.3 Results . . . . . . . . . . . . . . . . . . . . . . . . 133

7.3 .1 Password Use . . . . . . . . . . . . . . . . . . . . 133

7.3.2 Choosing Passwords . . . . . . . . . . . . . . . . . . 134

7.3 .3 Password Reuse . . . . . . . . . . . . . . . . . . . . 138

7.3 .4 Saving Passwords . . . . . . . . . . . . . . . . . . . . . 140

7.3.5 Changing Passwords . . . . . . . . . . . . . . . . 143

7.3.6 Security Perception . . . . . . . . . . . . . . . . . . 146

7.3.7 Demographic Trends . . . . . . . . . . . . . . . . . . . . . . 149

7.3 .8 Age . . . . . . . . . . . . . . . . . . . 150

7.3 .9 Security Expertise . . . . . . . . . . . . . . . 150

7.4 Discussion . . . . . . . . . . . . . . . . . . . . . . . . . . . 153

7.4 .1 Rationing . . . . . . . . . . . . . . . . . . . 154

7.4 .2 Password Management Tools . . . . . . . . . . . . . . 155

7.4 .3 Limitations . . . . . . . . . . . . . . . . . . 155

7.5 Conclusion . . . . . . . . . . . . . . . . . 156

8 A Password Manager that Doesn't Remember Passwords $\quad 157$

8.1 Introduction . . . . . . . . . . . . . . . . . 157

8.2 Password Coping Techniques . . . . . . . . . . . . . . . . . . 158

8.3 A New Model for Passwords . . . . . . . . . . . . . . . . . . 159 
8.3.1 The Cue Model . . . . . . . . . . . . . . . . . . . . . . . . . 159

8.3 .2 The Category Model . . . . . . . . . . . . . . . . . . . . 160

8.4 Versipass Prototype . . . . . . . . . . . . . . . . . . . . . . . 161

8.4.1 Image PassTiles . . . . . . . . . . . . . . . . . . . . . . 162

8.4.2 The Versipass Password Manager . . . . . . . . . . . . . 163

8.5 Versipass Security . . . . . . . . . . . . . . . . . . . . . . . 167

8.6 Usability Evaluation . . . . . . . . . . . . . . . . . . . . . . . . . 169

8.6 .1 Method . . . . . . . . . . . . . . . . . 170

8.6 .2 Results . . . . . . . . . . . . . . . . . . . . 171

8.7 Discussion . . . . . . . . . . . . . . . . . . . . . . . . . . . 175

8.7.1 Mental Models of Versipass _. . . . . . . . . . . . 175

8.7.2 Memory and Passwords . . . . . . . . . . . . . . . . . 177

8.7.3 What is a Password? . . . . . . . . . . . . . . . 178

8.8 Conclusion . . . . . . . . . . . . . . . . . . . . . . . . . . . . 179

9 Conclusions 181

9.1 Thesis Summary . . . . . . . . . . . . . . . . . . . . . . . . . . . 181

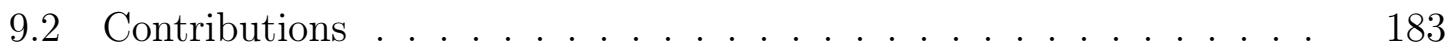

9.3 Limitations . . . . . . . . . . . . . . . . . . . . . . . . . 184

9.4 Future Research Directions . . . . . . . . . . . . . . . . . 185

$\begin{array}{ll}\text { References } & 187\end{array}$

$\begin{array}{ll}\text { Appendices } & 201\end{array}$

A Results of MA Memory Studies $\quad 202$

A.1 Introduction . . . . . . . . . . . . . . . . . . . 202

A.2 Results . . . . . . . . . . . . . . . . . . . . . . . . . 202

A.2.1 Study $1 \ldots \ldots \ldots \ldots \ldots \ldots$

A.2.2 Study $2 \ldots \ldots \ldots \ldots \ldots \ldots$

B Password Life Cycle Questionnaire Script 220

B.1 Demographics . . . . . . . . . . . . . . . . . . . . . 220

B.2 Passwords . . . . . . . . . . . . . . . . . . . . . . 221

B.2.1 Existing Passwords . . . . . . . . . . . . . . . . . 221

B.2.2 Setting up a New Account . . . . . . . . . . . . . . . . . 223

B.2.3 Changing a Password . . . . . . . . . . . . . . . . . . 223

C Life Cycle Survey Questionnaire $\quad 224$

C.1 Password Use . . . . . . . . . . . . . . . . . . . . . . . . . . . . . . . . . . . 224

C.2 Choosing Passwords . . . . . . . . . . . . . . . 226

C.3 Password Reuse . . . . . . . . . . . . . . . . . . . . . . . . 228

C.4 Saving Passwords . . . . . . . . . . . . . . . . . . . . . . 230

C.5 Password Changes . . . . . . . . . . . . . . . . . 232 
C.6 Password Security . . . . . . . . . . . . . . . . . . . . . . 233

C.7 Demographics . . . . . . . . . . . . . . . . . . . . . 234 


\section{List of Tables}

2.1 A summary of findings from different studies of password use. . . . 13

3.1 Customizable features of PassTiles. . . . . . . . . . . . . . 45

3.2 Theoretical password spaces for various configurations of the PassTiles password system. . . . . . . . . . . . . . 50

4.1 Password spaces for the configurations of the password systems used in the study. . . . . . . . . . . . . . . . . . . 57

4.2 Memory time statistics (hours) (Study 3). . . . . . . . . . . 64

$4.3 t$-tests of memory time (Study 3). . . . . . . . . . . . 64

4.4 Password reset statistics (Study 3) . . . . . . . . . . . 67

4.5 Wilcoxon tests of password resets comparing the assigned text condition with each of the graphical password conditions (Study 3). . . . 67

4.6 Login time statistics (seconds) (Study 3) . . . . . . . . . . . 67

4.7 Pairwise post-hoc Wilcoxon tests of login times for assigned password conditions using Bonferroni adjustment. . . . . . . . . . . . 69

4.8 Memory time statistics (hours) (Study 4). . . . . . . . . . . . 71

4.9 Password reset statistics (Study 4) . . . . . . . . . . . 71

4.10 Pairwise Wilcoxon tests of memory time using Bonferroni adjustment (Study 4). . . . . . . . . . . . . . . . . . . . . . 73

4.11 Login time statistics (seconds) (Study 4) . . . . . . . . . . 73

4.12 Pairwise Wilcoxon tests of login times using Bonferroni adjustment (Study 4). . . . . . . . . . . . . . . . . . . 74

4.13 Login time statistics (seconds) (Study 5) . . . . . . . . . . 76

4.14 Summarized results of our five studies of memory retrieval. . . . . . 78

5.1 Codes used in the open coding process. . . . . . . . . . . . . . 89

5.2 Life cycle example. . . . . . . . . . . . . . . . . . 110

7.1 Password use statistics for all respondents. . . . . . . . . . . . . . . 134 


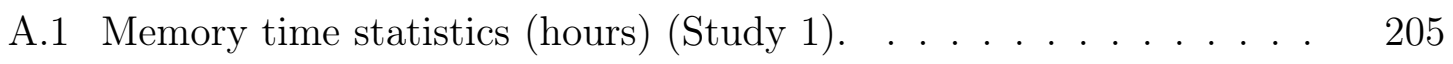

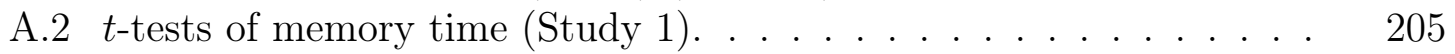

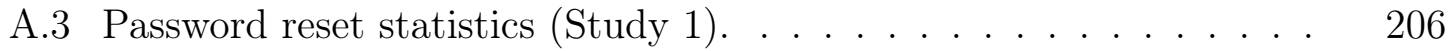

A.4 Login time statistics (seconds) (Study 1) . . . . . . . . . 208

A.5 Pairwise Wilcoxon tests of login times using Bonferroni adjustment (Study 1) . . . . . . . . . . . . . . . . . 210

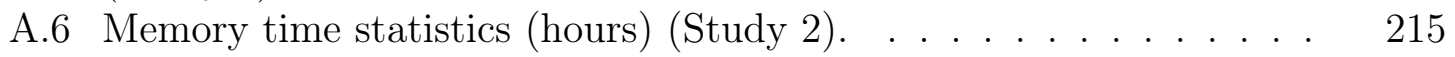

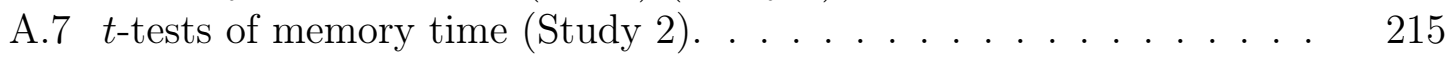

A.8 Password reset statistics (Study 2) . . . . . . . . . . 215

A.9 Wilcoxon tests of password resets comparing the assigned text condition with each of the graphical password conditions (Study 2) . . . 217

A.10 Login time statistics (seconds) (Study 2) . . . . . . . . . . . 217

A.11 Pairwise Wilcoxon tests of login times using Bonferroni adjustment (Study 2) . . . . . . . . . . . . . . . . . . . . . 219 


\section{List of Figures}

2.1 The Draw-A-Secret password grid, showing a sample password. . . . 28

2.2 The PassPoints login screen. . . . . . . . . . . . . . . . . 28

2.3 The password creation screen for PCCP. . . . . . . . . . . . . 29

2.4 The Passfaces login screen. . . . . . . . . . . . . . . . . . 30

3.1 The Blank PassTiles password creation screen. . . . . . . . . . . . . 42

3.2 Password creation interface for Object PassTiles. . . . . . . . . . . 44

3.3 Password creation interfaces for two different grid and password length configurations of Image PassTiles. . . . . . . . . . . . . . . . . . 46

3.4 Password creation interfaces for two variations of Image PassTiles. . 47

3.5 Password creation for a user-selected, digit login PassTiles password. 48

4.1 One of the three websites used in the studies, showing the PassTiles password creation screen. . . . . . . . . . . . . 56

4.2 Distributions of memory time (in hours) by number of participants for each study condition (Study 3). . . . . . . . . . . . . 65

4.3 Distributions of password resets by number of participants for each study condition (Study 3). . . . . . . . . . . . 66

4.4 Distributions of login times by number of participants for each study condition (Study 3). . . . . . . . . . . . . . . 68

4.5 Stacked bar plots showing frequency of reported password recording. $\quad 70$

4.6 Distributions of memory time (in hours) by number of participants for each study condition (Study 4). . . . . . . . . . . . . . . 72

4.7 Distributions of password resets by number of participants for each study condition (Study 4). . . . . . . . . . . . . . . . 72

4.8 Distributions of login time by number of participants for each study condition (Study 4). . . . . . . . . . . . . . . 73

4.9 Heatmaps showing the spatial positioning of clickpoint entries in PassTiles. $\quad 75$

4.10 Distributions of login time (in seconds) by number of participants for each study condition (Study 5). . . . . . . . . . . 77 
5.1 Website screenshot cards. . . . . . . . . . . . . . . 83

5.2 Rearranging the codes to look for patterns in the axial coding process. 94

5.3 A detail of one of the websites used as a prop in our interviews. . . 100

5.4 The password life cycle. . . . . . . . . . . . . . . . . 108

7.1 Responses to rating scale questions about how frequently respondents include various types of personal information in their passwords. . .

7.2 Responses to rating scale questions about how frequently respondents include various types of personal information in their passwords. . .

7.3 Density grid showing the frequency of pairs of co-incident elements that respondents include in their passwords. . . . . . . . . . 137

7.4 The reasons that respondents reuse passwords. . . . . . . . . . 138

7.5 Responses to rating scale questions asking about how respondents reuse passwords. . . . . . . . . . . . . . . . . . . . 139

7.6 Frequency with which respondents reported writing passwords down. 140

7.7 Density grid showing the frequency of pairs of co-incident types of passwords that respondents write down. Darker colours indicate higher frequency, and the diagonal boxes show the total frequency of selections for that element. For example, respondents most often write down infrequently-used passwords, and the most frequently written down combination is infrequently-used and high-security passwords.

7.8 Types of passwords that users do not save in the web browser. . . .

7.9 Responses to rating scale questions about how frequently users use password managers. . . . . . . . . . . . . . . . . . . 142

7.10 Types of events that motivate users to change their passwords. . . . 144

7.11 Rating scales showing users' strategies for forced password changes. 144

7.12 Rating scales showing users' coping strategies for handling forgotten passwords. . . . . . . . . . . . . . . . . . . . 145

7.13 Rating scales showing users' behaviour when resetting forgotten password changes. . . . . . . . . . . . . . . . . . . . 146

7.14 Rating scales showing perceptions of difficulty of security tasks. . . 147

7.15 Rating scales showing perceptions of behaviour across time. . . . . 148

7.16 Rating scales showing perceptions about attention paid to security. 149

7.17 Rating scales showing how older and younger users reuse passwords. 151

7.18 Rating scales showing how security experts use password managers. 152

7.19 Barplots showing why experts and non-experts do not use password managers. . . . . . . . . . . . . . . . . . 153

8.1 The cue model. . . . . . . . . . . . . . . . . . . . . . . . . . . . . . 159

8.2 The category model. . . . . . . . . . . . . . . . . 160

8.3 The password creation interface for Image PassTiles. . . . . . . . . 162

8.4 A sequence diagram of the login process using Versipass. . . . . . . 163 
8.5 Adding a new account and password category to Versipass (continued on next page). . . . . . . . . . . . . . . . . . . . . . . . . . 164

8.6 Logging in using Versipass. . . . . . . . . . . . . . . 166

A.1 Distributions of memory time (in hours) by number of participants for each study condition (Study 1). . . . . . . . . . . . .

A.2 Distributions of resets by number of participants for each study condition $($ Study 1). . . . . . . . . . . . . . . . 207

A.3 Distributions of login time (in seconds) by number of participants for each study condition (Study 1). . . . . . . . . . . . . 209

A.4 Stacked bar plots showing frequency of reported password recording. 212

A.5 Distributions of memory time (in hours) by number of participants for each study condition (Study 2). . . . . . . . . . . . .

A.6 Distributions of password resets by number of participants for each study condition (Study 2). . . . . . . . . . . . . . . .

A.7 Distributions of login times by number of participants for each study condition (Study 2). . . . . . . . . . . . . . . 


\section{Chapter 1}

\section{Introduction}

\subsection{Research Problem}

Passwords present multiple difficulties for users. Good passwords are difficult for users to create, difficult to remember, and users must keep track of large numbers of passwords. Often, the passwords that are easy for users to remember are also easy for attackers to guess. This is known as the password problem [147]. As more activities take place online and require user accounts with credentials, the scale of the password problem increases. Users have more passwords to remember and track, and the potential consequences of a breach become higher as more information is stored online.

Users are often regarded as the weak link in security systems because they do not follow advice or comply with security rules. However, Adams and Sasse [1] point out that users are not "inherently insecure"(p. 43). Rather, many security systems are poorly designed for users, and these poor decisions lead users to avoid or misuse the systems [1]. Security systems must be designed so that legitimate users find them straightforward and easy to use, but malicious users (attackers) find them impenetrable. The presence of the adversary deeply complicates the creation of usable security systems.

Security has a number of properties that effectively prevent users from succeeding by simply trying harder [146]. Rather than being the reason that users sit down at the 
computer, security is typically a secondary task that prevents the user from completing their primary task. This means that users are typically not strongly motivated to pay attention to security. In addition, security is an abstract and complex subject that involves many hidden variables, making it hard for many end users to understand. The requirements of secure systems also impede usability: security systems cannot provide feedback to users because of attackers, and since systems are only as secure as their weakest link, users must be vigilant to every aspect of security. If a system has been left unprotected for any period of time, the security of the system cannot be guaranteed.

Many ideas to address the password problem have been proposed, including biometrics [35], single sign-on [130], graphical passwords [11], and password managers [120]. Some ideas are aimed primarily at improving passwords (e.g., pass-phrases [117] and pronounceable passwords [56]). However, none of the current approaches have supplanted traditional passwords as the most prevalent form of credential. Herley and van Oorschot [65] point out that passwords are cheap, and familiar to most users. They are easily revoked, and password systems can be designed so that password changes require no administrator intervention. Although claims have been made for at least the last decade that passwords are dead or outdated (including Bill Gates' 2004 statement that "the password is dead" [81]), passwords are widely used today.

In this thesis, we explore practical solutions to the password problem, and investigate questions about password alternatives. We are specifically interested in end users, and how authentication affects them and their daily lives. Passwords affect users in the most personal of ways - protecting financial information, personal communication, social interactions, and many other activities. They are woven into everyday life, and form a major management challenge for many users.

Although much research on passwords exists and is ongoing, there remain open questions about how we can best support users and their passwords. These include questions about passwords and memory: how can passwords be best designed to take advantage of the strengths of human memory? How can we use what we know about memory and human cognition to improve the design of passwords and help make randomly assigned passwords more memorable?

Since most people simply cannot remember very much randomness, the demands of passwords on users are practically impossible to fulfill [53], and most users have 
adopted limited strategies to cope with passwords. What kind of trade-offs do users make? Where do they compromise, and where do they invest their limited energies? What motivates users' coping strategies? Can we leverage what users are already doing to help them be more secure without having to significantly change their behaviour or invest in new strategies?

Finally, how can we take research on passwords and practically apply it to the design of password management tools?

\subsection{Contributions}

The contributions of this thesis investigate practical aspects of addressing the password problem. We use a variety of methodological approaches to directly study users, and better understand their abilities, limitations, knowledge, and coping strategies.

These include:

- An exploration of how assigned random passwords can be made more memorable. Random assigned passwords are more secure than user-chosen passwords, but users typically have trouble remembering random passwords. We use knowledge from the psychological literature about memory in the design of a graphical password scheme that supports usable random password assignment. The scheme, PassTiles, has a variety of flexible features that can be used to customize the system to the particular usability and security requirements of different situations.

- An empirical investigation into how graphical passwords should be designed to best take advantage of the capabilities of human memory. We used the PassTiles graphical password system to explore what kind of memory retrieval should be leveraged to best support random assigned passwords. We conducted three user studies with 178 participants to investigate how memory retrieval affects the usability and memorability of assigned random graphical passwords. We find that recognition memory does best support the memorability of passwords, but that there are associated usability issues that must be carefully handled in the design of password systems. 
- A qualitative study of users' coping strategies for password management. We analyzed interview data using Grounded Theory, and found that users' password behaviour over time develops their passwords into a life cycle as users adapt to changing circumstances and demands. We find that users' coping strategies are rational in spite of their limitations, and we use the life cycle to suggest design recommendations for password technologies.

- A qualitative exploration of experts' coping strategies for password management. We interviewed computer security experts about how they cope with the demands of password management, and found that they combine multiple strategies to strongly protect certain accounts while not over-investing in less important accounts. The expert strategies can be used to recommend password management techniques for all users.

- A survey-based study that examines how the life cycle model holds up in the larger population. The survey showed that most users use complex coping strategies to manage passwords, and that although they do ration their efforts differently for different accounts, they do not perceive this task as a hardship.

- The design of a new approach to password management. We designed Versipass, a login manager that takes advantage of users' existing coping strategies and integrates them with graphical passwords and password managers.

\subsection{Organization of Thesis}

The first person plural voice $(w e)$ is used in this thesis to reflect the fact that the work was conducted by me under the supervision of Robert Biddle. The rest of the thesis is organized as follows:

- Chapter 2 presents background and related work for the topics covered in the thesis. These include memory, graphical passwords, and password managers.

- Chapter 3 introduces the PassTiles graphical password system. We designed PassTiles so that memorable passwords could be easily assigned, and PassTiles has a variety of customizable features that are discussed in the chapter. 
- Chapter 4 presents the results of a series of studies investigating how leveraging different types of memory retrieval in the design of graphical passwords affects their memorability.

- Chapter 5 presents a qualitative study of users' coping strategies for password management. Grounded Theory is used to analyze interviews about password management, and a theory of password management emerged from the data.

- Chapter 6 extends the work in Chapter 5 by exploring the password management practices of computer security experts to see how they relate to the coping strategies of non-experts.

- Chapter 7 presents the results of a survey exploring how the life cycle model applies to a broader population.

- Chapter 8 introduces Versipass, a prototype password manager that allows users to use secure and memorable graphical passwords on any account, securely reuse passwords, and better manage their passwords without becoming a single point of failure.

- Chapter 9 describes our conclusions and future research directions.

\subsection{Related Publications}

Much of the text of this thesis is adapted or taken from publications containing this work. These include (organized by their relevance to work in this thesis):

- Stobert, E. \& Biddle, R. (2013). Memory Retrieval and Graphical Passwords. In Proceedings of the 9th Symposium on Usable Privacy and Security (SOUPS). Newcastle, UK: ACM.

This paper (incorporated here in Chapter 4 and Appendix A) includes five studies. The first two studies were done as part of my MA (Psychology, 2011), and were inconclusive; they are included here to give context and clarity. The later studies were conducted as part of my PhD. These explore the topic in more depth, and show the link between the password design strategies compared and the consequent memorability. 
- Stobert, E. \& Biddle, R. (2013). Authentication in the Home. In Workshop on Home Usable Privacy and Security, held as part of SOUPS '13. Newcastle, UK.

This workshop paper investigated how relationships in the home affect authentication, specifically password sharing and security organization. Work on password sharing in the background of this thesis (Chapter 2) was taken from this paper.

- Stobert, E. \& Biddle, R. (2014). The Password Life Cycle: User Behaviour in Managing Passwords. In Proceedings of the 10th Symposium on Usable Privacy and Security (SOUPS). Menlo Park, USA: USENIX Association.

This paper investigated how users cope with password requirements and presented a Grounded Theory analysis of a series of interviews asking questions about password management issues. This work is included here in Chapter 5.

- Stobert, E. (2014). The Agony of Passwords: Can We Learn From User Coping Strategies? (Extended Abstract) In Proceedings of the Conference on Human Factors in Computing Systems (CHI), Toronto, Canada: ACM.

This extended abstract was a semi-finalist in the 2014 CHI student research competition. It presented a thematic analysis of early interview data about password management that was substantially extended and published at SOUPS 2014 (and appears here in Chapter 5).

- Stobert, E., \& Biddle, R. (2014). A Password Manager that Doesn't Remember Passwords. In Proceedings of the 2014 Workshop on New Security Paradigms $(N S P W)$. Victoria, Canada: ACM.

This work presented an in-depth analysis of Versipass, including discussion of the system, security analysis, and usability evaluation. Chapter 8 covers this work.

- Stobert, E. \& Biddle, R. (2012). Visual End-User Security. Presented as part of the graduate consortium at the Symposium on Visual Languages and Human-Centric Computing (VL/HCC). Innsbruck, Austria: IEEE.

This short paper gave an early overview of Versipass (then called "I Can't Believe it's a Password Manager") at the VL/HCC graduate consortium. 
In addition, I have been an author on a number of other publications which have indirectly contributed to this thesis. These include:

- Chiasson, S., Deschamps, C., Stobert, E., Hlywa, M., Freitas Machado, B., Forget, A., Wright, N., Chan, G., \& Biddle, R. (2012). The MVP Web-based Authentication Framework. In Financial Cryptography and Data Security (FC). Bonaire: Springer.

This short paper presented the MVP framework, developed by members of our lab to make it easier to conduct more ecologically valid password studies. I contributed to the framework by developing administrative tools for MVP, and helped test the framework in several initial studies. I also collaborated on paperwriting, and provided data and analysis to the user studies highlighted in the paper.

- Chiasson, S., Stobert, E., Forget, A., Biddle, R., \& van Oorschot, P.C. (2012). Persuasive Cued Click-Points: Design, Implementation, and Evaluation of a Knowledge-Based Authentication Mechanism. IEEE Transactions on Dependable and Secure Computing (TDSC), 9(2):222-235.

This journal paper brought together several studies on Persuasive Cued ClickPoints (PCCP). A study of PCCP conducted for my undergraduate honours project was included in the paper, and I also assisted in the editing. My work on PCCP led to my work on PassTiles (Chapter 3), which attempts to address some of the remaining usability and security issues present in PCCP. 


\section{Chapter 2}

\section{Background and Related Work}

\subsection{Authentication}

Authentication is the act of verifying that a user is who they claim to be [90], and is a key topic in computer security. Authentication is different than authorization, which gives a certain user permission to access materials or carry out actions. A variety of authentication methods exist, including text passwords, smartcards, and fingerprint readers. These different methods of authentication may be distinguished into three categories based on the characteristic that is being verified [90]. The authentication event may be based on something the user knows (e.g., passwords, PINs), something the user has (e.g., smartcards, password-generation tokens), or something the user is (either physical or behavioural biometrics, e.g., fingerprints, retinal scans, typing patterns).

While the purpose of authentication is to provide security, its usability cannot be ignored. If systems are not usable, users will misuse or avoid the system, leading to security failures [1]. Thus, if an authentication system is not usable, it cannot be secure. Exploring the usability of security systems is a relatively new area of research, combining research from human-computer interaction with computer security to understand how the human element affects the security of the system. 
Security systems have a number of properties that can create usability problems. Whitten and Tygar [146] identified five properties of security that complicate designing for usability:

- Security is a secondary task for most users - it is not the purpose for which they sit down at the computer, and as such, they are unmotivated to pay it more attention.

- Security is an abstract topic, and security tasks can be difficult for non-expert users to understand.

- Security software is unable to give useful feedback, because it might be helpful to attackers as well as legitimate users.

- Security suffers from the "barn door property" - once a system has been left unprotected for any amount of time, we cannot be sure that the system has not been attacked. It is thus important that users master security very quickly to avoid creating vulnerabilities while they are learning.

- A system can only be as secure as its weakest link, so users must always attend to all aspects of security.

Although biometrics and token-based authentication exist and show promise, we focus here on knowledge-based authentication. In this chapter, we review the literature concerning passwords and usable authentication. We begin by outlining information on how password security is assessed. In the later sections, we provide background on the problems with passwords, the realities of life with passwords for users, and work that has been done to address the password problem. Finally, we provide some results from the psychological literature on memory that may be helpful to understand the usability issues for users with passwords.

\subsubsection{Password Security}

Attacks on authentication systems can be divided into two classes: guessing attacks,

and capture attacks [11]. Guessing attacks take place when the attacker attempts to gain access to an account by guessing the password. They include brute force 
attacks, where the attacker systematically guesses every possible password, and dictionary attacks, where the attacker begins with a dictionary of probable passwords and prioritizes their guesses based on the likelihood of success. Guessing attacks may take place either online (where the lockout policy on entry attempts protects accounts) or offline with a stolen password file (where best practice recommends that passwords be salted and hashed for secure storage). In contrast, capture attacks are those where an attacker attempts to learn a complete password by recording it or tricking the user into disclosing it. Capture attacks include malware attacks, where the password is recorded through the installation of malicious software (e.g., a keylogger); recording attacks, where the attacker spies on password entry (e.g., recording PINs with a camera); phishing attacks, where the attacker tricks the user into sharing their password on another website; and network attacks, where an attacker learns passwords by spying on network communications.

When evaluating the security of knowledge-based authentication schemes against guessing attacks, security is often measured by the size of the password space, or the number of passwords that can be created in the system. The theoretical password space, which is the set of all possible passwords, is further distinguished from the effective password space, which is the set of passwords that users actually select. The effective password space is often much smaller than the theoretical password space [16], and is usually distributed differently, with hotspots over popular passwords and gaps around unpopular passwords. Since all users' passwords are generally not known, it can be difficult to understand exactly how the effective password space is distributed.

Knowledge of the effective password space can be leveraged by attackers for more efficient attacks (e.g., by beginning a guessing attack with a password that is a known or suspected hotspot), and as such, one of the design goals for knowledge-based authentication is to evenly distribute users' password selections over the theoretical password space. A straightforward way to do this is to assign random passwords. However, randomly assigned passwords are often difficult to memorize and are disliked by users.

Threat models are created to evaluate the risks that are expected to be relevant to an authentication system. When creating a threat model for knowledge-based authentication, a variety of issues relating to what the system will protect, and the 
context in which it will be used must be considered. The threats must also be modeled in relation to the design decisions, such as whether users will be allowed to select their own passwords.

\subsection{The Password Problem}

Passwords pose a considerable usability challenge for end users, who are asked to create secure, unique passwords for every account, remember each of those passwords for a long time, and remember which password goes with which account for multiple accounts. These security requirements place demands beyond human capability on users' memory, time, and attention, and lead users to create passwords which are memorable, but easily guessed by attackers. This is known as the password problem [147]: passwords that are easy to remember are also easy to guess.

The password problem has existed for most of the history of computing. Morris and Thompson [92] describe the problem in a 1979 article about passwords in the UNIX operating system. However, with the introduction of personal computing and the web, the problem has scaled enormously for most end users. Current research continues to find users creating weak passwords [16], and instances of attackers obtaining passwords and leading to major losses are increasingly common [46].

The password problem results from a mismatch between security expectations and users' abilities [147], and these disconnects can lead to the misuse or avoidance of security mechanisms [1]. Users may avoid password expectations by writing passwords down, or by reusing the same passwords across multiple accounts.

Conventional wisdom concludes that users are lazy and unwilling to comply with security advice [64]. Correspondingly, the conventional suggestion is that users should be motivated to try harder to follow security advice, and that we should better educate users about the dangers of poor security practices. However, the quantity of information that users are expected to remember is arguably impossible to memorize. Florencio, Herley and van Oorschot [53] observe that if a user has $N$ distinct accounts, and creates secure and distinct passwords for each with bit size $\log (S)$, the user will have to remember not only $N \times \log (S)$ bits, but also which of the $N$ ! mappings of password to account is correct. This gives a total expected bit size to be remembered 
of $E(N)=N \times \log (S)+\log (N !)$. The factorial term quickly grows very large as $N$ increases.

Herley also argues for the rationality of users' decisions to ignore security advice [64]. Not only are password expectations impossible for users to meet, but a cost-benefit analysis of following security advice suggests that users should not even try. System administrators are at least somewhat aware of this: websites that rely on popularity for their users typically have less restrictive password rules, regardless of the sensitivity of content [51]. This suggests that websites with more restrictive password policies do not necessarily have higher security needs, it is simply that they are less affected by the loss in usability that accompanies the restrictive policies.

Another problem for passwords is the divide between those who make security policies (including password policies), those who enforce security policies, and those who follow security policies. Studies of information security in organizations have revealed a "digital divide" between policy makers, who typically do not bear the cost of security vs. users, who handle the downsides of security, including lost productivity and opportunities [3]. Beautement, Sasse and Wonham [9] suggest that organizations must factor in and budget for the cost of employee compliance with security policies. They suggest that organizations need to consider costs and benefits to the organization when setting security policy.

Although numerous other authentication strategies have been proposed, including biometrics, smart cards or authentication tokens, passwords continue to be the most common form of authentication. However, passwords have a number of useful properties that contribute to their persistence [65]. Passwords are simple to implement, do not involve any extra hardware, and scale at no additional cost to websites. Passwords are easily revoked or reset in case of loss, and do not breach users' privacy. For the user, they are portable and convenient, as well as being familiar and easily understood. In contrast, biometrics have inherent privacy concerns, problems with revocation, and they usually require the expense and deployment of new hardware. Smart cards and authentication tokens are also expensive to deploy, and they require that the user remember to carry the token around. 
Table 2.1: A summary of findings from different studies of password use.

\begin{tabular}{|l|l|l|l|l|l|}
\hline Researchers & Year & Type of Study & $\begin{array}{l}\text { Average } \\
\text { Number of } \\
\text { Accounts }\end{array}$ & $\begin{array}{l}\text { Average } \\
\text { Number of } \\
\text { Passwords }\end{array}$ & $\begin{array}{l}\text { Presence } \\
\text { of Reuse }\end{array}$ \\
\hline Brown et al. & 2004 & Survey & 8.2 & 4.5 & Yes \\
\hline Gaw and Felten & 2006 & Survey & 12.0 & 3.3 & Yes \\
\hline $\begin{array}{l}\text { Florencio and } \\
\text { Herley }\end{array}$ & 2007 & $\begin{array}{l}\text { Instrumented } \\
\text { toolbar deploy- } \\
\text { ment }\end{array}$ & 25.0 & 6.5 & Yes \\
\hline Notoatmodjo & 2007 & Survey & 12.9 & 8.1 & Yes \\
\hline $\begin{array}{l}\text { Hayashi and } \\
\text { Hong }\end{array}$ & 2011 & Diary study & 11.0 & - & - \\
\hline $\begin{array}{l}\text { Grawemeyer and } \\
\text { Johnson }\end{array}$ & 2011 & Diary study & - & 7.9 & Yes \\
\hline Steves et al. & 2014 & Diary study & - & - & Yes \\
\hline
\end{tabular}

\subsection{Users and Passwords}

There has been extensive research conducted on users and passwords, investigating topics such as the number of passwords that users possess, password reuse, password recording, how users choose passwords, and the influence of password policies on how users select passwords. Understanding how users experience passwords may help us to better solve the problems that users have with passwords.

\subsubsection{Number of Passwords}

Numerous studies have investigated how many passwords and accounts that users possess in their everyday lives. Table 2.1 shows a summary of statistics from several different studies of passwords. Although different studies show different results, the studies agree that while users often have many accounts, they typically have far fewer unique passwords.

In 2004, Brown, Bracken, Zoccoli and Douglas [23] surveyed undergraduates and found that they had an average of 8.18 accounts and 4.45 passwords. A 2006 study by Gaw and Felten [57] found that undergraduates had an average of about 12 accounts, but they had fewer unique passwords and password reuse was rampant. They 
considered passwords that were the same except for appended characters to be part of password "families", and found that most participants only had about three password families. The study showed that most participants cited easier memorability as their reason for password reuse, and that participants classified their accounts by the desired level of privacy and security.

Florencio and Herley [50] conducted a large scale study of password use through the six-month deployment of a Microsoft toolbar. They collected data from more than 250,000 users, and found that the average user had 6.5 passwords, each of which was shared across 3.9 websites. They found that the average user accessed 25 accounts over the six month period, and logged into eight accounts per day.

Notoatmodjo [98] conducted detailed survey-based research on users' passwords. He found that participants reported an average of 12.9 accounts and 8.1 passwords, and found that number of accounts was correlated to the user's number of years of internet experience. He had users classify their accounts into high- and low-importance account groups and found that this categorization was related to the length of passwords, and how they were reused. Passwords for high-importance accounts were reused much less frequently (only $45 \%$ of passwords for high-importance accounts were reused vs $96 \%$ for low-importance accounts).

A 2011 diary study of password use by Hayashi and Hong [63] collected detailed records of password entries over a two-week period. They found that users accessed a mean of 8.6 accounts over two weeks, and estimated that most participants had about 11 accounts in total. Although they did not study password reuse directly, all of their participants reported reusing passwords across multiple accounts.

Grawemeyer and Johnson [59] conducted a seven-day diary study of password use in the UK, and found that the average user had 7.95 passwords. Their study collected a total of 175 passwords, of which only 69 were unique and not reused across multiple accounts. In their study, most accounts were categorized as either work-related or email.

Steves et al. [123] conducted a large-scale diary study of authentication events in an organizational setting. They found that on average users authenticated 23 times a day, and were frustrated by the frequent disruptions to their primary tasks. They examined when users authenticated, and found that authentications peaked around 
the beginning of the day, but were otherwise evenly distributed. Reflecting their study setting, they found that the majority of reported authentication events were work-related.

We observe that many of these studies are now out-of-date, and we speculate that more modern users may have more passwords and accounts than are reflected here.

\subsubsection{Password Choice}

Another topic of interest for research on the security of passwords is the kind of passwords that users actually choose. Users are often advised to choose long passwords that will be easy for them to remember, but hard for others to guess. However, these kinds of passwords can be difficult for users to choose, and users may not have a good understanding of what would be hard for another to guess.

A 1979 study by Morris and Thompson [92] gathered passwords from users of the UNIX operating system. They collected 3289 passwords, and found that $86 \%$ of them could be easily guessed by attackers (i.e., the passwords were either very short, or were dictionary words). In 1989, Riddle, Miron and Semo [109] assessed passwords from their university's timesharing environment. They gathered 7014 passwords and analyzed their length, character composition, and semantic categorization. They found that the majority of passwords had five or fewer characters, and contained only lower-case letters and no digits. Semantic information found in passwords included first names, license plate numbers, room numbers, telephone numbers, and building room numbers. Zviran and Haga [152] surveyed employees of the US Department of Defence in 1999, and found that the average length of passwords was six characters, and that $80 \%$ of respondents reported a preference for alphabetic characters.

More recent studies have confirmed these findings, and there is general evidence that users frequently include personal information in passwords [44, 152, 118]. This can lead to security vulnerabilities because personal information can often be easily guessed. Brown et al. [23] surveyed undergraduate's passwords and found that $66 \%$ of passwords involved personal information, and almost half of all sampled passwords involved either a birthday or a proper name. Grawemeyer and Johnson [59] found that users cope with the demands for secure passwords by integrating personal or account-specific information into passwords, and using patterns in passwords. Shay 
et al. [118] found that users frequently based their passwords on words, names, or public information (e.g., addresses, phone numbers).

\section{Password Datasets}

Finding or collecting password datasets has traditionally been a hurdle for studying users' password choices. However, a number of password datasets have been leaked in recent years, providing researchers with an ecologically valid set of passwords to study. One of the most well-known datasets is the RockYou dataset, which includes 32 million passwords stolen from rockyou.com in an SQL injection attack in December 2009 [141, 39]. Other leaked datasets have emerged from attacks on Twitter [131], Yahoo [7], and LinkedIn [145]. Although the ethics of using leaked datasets for research are debatable [47], the RockYou dataset (and others) have provided insight into users' real life password choices.

Analysis of the RockYou dataset [141] showed the average length of passwords was 7.88 characters, and that "1" was by far the most likely digit to be included in passwords. The distribution of special characters was more even, with exclamation points, underscores, and full stops accounting for $43.87 \%$ of symbols. Digits and special characters were most likely to be appended at the end of passwords, and digits were more likely to follow symbols than vice versa.

Another analysis of the RockYou dataset [18] looked at 4-digit PINs in the set. These passwords were more likely to occur in year or data ranges (i.e., ranging from $\approx 1945$ to 2012), and that hotspots in the set corresponded to popular passwords such as "1234". The study also analyzed a set of iPhone unlock codes (collected from the Big Brother Camera Security app [5]) and found that those codes were likely to contain geometric patterns stemming from the arrangement of the digits on the unlock screen.

Another focus of the analyses of large datasets has been evaluating the strength of passwords against guessing attacks, and optimizing those attacks for better guessing.

Weir et al. [141] analyzed the RockYou dataset and found that the NIST guidelines for estimating password entropy [24] did not provide a realistic estimate of the guessability of the passwords. They showed that factors such as password length, placement of digits and symbols, and likely digit choices can be exploited by attackers for more effective guessing. 
An alternative to studying leaked databases is to study anonymized password sets. Bonneau [16] developed a methodology for hashing passwords at the time of collection with a secret key that is subsequently destroyed. This preserves users' privacy, while maintaining the password distribution for study. Bonneau used this method to study a dataset of nearly 70 million Yahoo! users. Bonneau's analysis [16] studied a range of sub-populations of Yahoo! users, and investigated how attacks can be optimized based on a range of factors, including the purpose of the website, selected language, age, and gender. He found that dictionaries can be optimized to better guess passwords for different demographic groups, and that dictionary specification can lead to $25 \%$ improvement in guessing. He also found "global passwords" which occurred frequently in all groups, and that the most common global password occurred with $P \geq 0.14$ in all groups.

Mazurek et al. [89] collaborated with the information technology division at Carnegie Mellon University to obtain a set of more than 25,000 passwords from users of the university infrastructure. They evaluated the security of the password set by evaluating its resistance to offline guessing attacks. Their analysis confirmed previous assumptions that adding digits, symbols, and upper case characters to passwords increases resistance to guessing attacks. They also drew conclusions about how artificiallygenerated datasets can reflect real password datasets, and suggest that passwords created in controlled studies can be a valid substitute for real world data.

\section{Factors Affecting Password Composition}

A number of contextual factors affect how users create and choose passwords.

Yan et al. [148] experimented with how different types of password advice affected the security of users' passwords. They gave one group standard password advice (at least seven characters, with one non-letter character), one group a method for choosing a random eight character password, and another group was told to choose a password based on a mnemonic phrase (a passphrase). In their attacks, $32 \%$ of passwords from the control group were cracked, while only $6 \%$ of the passphrases and $8 \%$ of the random passwords were guessed. However, participants reported having more trouble remembering their random passwords, leading the authors to conclude that users should be advised to create passphrases. 
Password composition policies also influence how users select passwords. A study of a change in password policy at Carnegie Mellon University found that the shift to a more complex policy annoyed and frustrated users, causing them to rely on new coping strategies, but also made them believe that they were more secure [118]. The new policy caused users to take more attempts to create a compliant password, and users reported that they were more likely to have forgotten the new passwords.

Another study of 5000 users of Amazon's Mechanical Turk found that password policies influence how users choose passwords [80]. It showed that manipulating the features of password creation policies, including length, required character sets, and dictionary checks, did affect the entropy of created passwords. The study also found that the policies that generated the most entropy required either a variety of special characters or a long minimum length, as well as a dictionary check at password creation. However, participants in this study found these passwords harder to create and remember.

Password strength meters are a tool sometimes provided to help users create more secure passwords. The meter assesses the security of the password at create time, and gives the user immediate feedback about the security of their password. Often, these meters use a stoplight metaphor with red for poor security, yellow for mediocre security, and green for good security. They may or may not give feedback about how users can improve their passwords. Ur et al. [135] experimented with a variety of strength meters and found that the presence of any strength meter caused participants to create longer passwords, and to include more digits. They did not find that all password meters positively affected the strength of created passwords against guessing attacks. Another study of password meters [49] found that the presence of any meter was more important than the design of the meter itself, but that the effect of the meter could be heavily context-dependent, and that meters did not discourage users from reusing weak passwords.

\subsubsection{Password Reuse}

One coping strategy for having multiple passwords is to reuse passwords across multiple accounts. This strategy is widely employed by users, but has security risks. If a reused password is discovered (e.g., through a leaked password set), an attacker may be able to gain access to several accounts [68]. 
Almost all studies of password use have uncovered password reuse among users [152, $57,50,63,118,98]$. Notoatmodjo [98] found that reuse increased with the number of users' accounts, and that most users cited increased memorability as the reason for reusing passwords. Reuse is a simple and intuitive coping technique that scales well to handling password meters [47], and coping with password policies [118].

Empirically tracing the extent of password reuse can be difficult. Das et al. [40] examined leaked datasets from 10 websites, and found that $43 \%$ of all passwords in their data set were reused across multiple accounts. They showed that knowledge of password reuse (via cross-referenced userIDs) can be leveraged for more efficient password attacks.

Even when users do not completely reuse passwords, they often reuse pieces of passwords, or make minor modifications when using a password on another website. Studies $[40,141]$ have found that most transformations take place at the beginning or end of a password, and that the most common transformations are to add a number, symbol, or capitalization to comply with a new password policy.

von Zezschwitz, De Luca and Hussman [138] interviewed users to trace reuse over the lifetime of passwords. They found that users are likely to retain fragments of existing habits and passwords across the creation of new accounts and changes in policy, leading to long-term reuse.

While "strict" security advice tells users not to reuse passwords, anecdotal evidence suggests that password reuse is the technique used by many security experts to manage passwords. Norman describes his experience asking security professionals about their personal password strategies:

"What do security professionals do? I asked attendees at the security conferences. Many of the security experts said they do "what everybody does: have two passwords.' " (p. 63, [97])

There has been little work investigating how to make password reuse safer, but Blocki, Blum, Datta and Vempala [15] propose a human-computable password scheme that remains secure even if an attacker is able to observe multiple logins using the scheme. 


\subsubsection{Password Recording}

Another coping strategy that users adopt for remembering passwords is to write passwords down. Writing passwords down can allow users to select and remember more complex passwords, as well as a higher number of passwords, but can have security risks if an attacker were to discover the list of recorded passwords.

Studies have found that many users write down some or all of their passwords. Zviran and Haga [152] asked users about their password recording practices, and found that $35 \%$ of their participants wrote down their passwords, and the most common storage locations for recorded passwords were wallets, notebooks, and calendars. They found no relationship between password characteristics (length, composition) and likelihood of password recording, but did find that participants were significantly more likely to write down passwords that were difficult to remember, or that they used infrequently. Grawemeyer and Johnson [59] found that writing passwords down was a coping strategy used to complement password reuse. They found that the probability of a user writing a password down was almost 18 times higher if the password was unique.

The conflict between "difficult to guess" and "easy to remember" can lead to the worst case in maintaining security - the user writing it down. Riddle, Miron \& Semo, 1989 [109]

Writing passwords down (or recording them in a similar format) is a common coping strategy for memorizing passwords. Although writing passwords down was once held as a worst case scenario (as in the quote from Riddle et al.), experts have more recently advocated that users write their passwords down. A well-known security expert advocates for writing passwords down on the basis that most users are better able to protect a list of (secure) written passwords than remember secure passwords for each site [114].

An important issue for recorded passwords is how they are stored: if securely stored, writing passwords down can be a perfectly acceptable technique for aiding users with passwords. Shay et al. [118] asked users how they protected their recorded passwords and found that about $30 \%$ of people did not protect them at all. Of the remaining $70 \%$, strategies were varied, but included hiding it, or storing it on another computer or device with a password. 


\subsubsection{Password Sharing}

Passwords are usually only intended for one user, but often end up shared between users. Although password sharing is viewed as a security breach and discouraged by security experts and security policies [88], passwords are shared by users as an expression of trust, as a necessity of having multiple users use a system, or because of other realities of daily life (such as disability).

In the context of the home, different trust relationships affect the ways that people choose to share their lives, and their passwords. These relationships may include romantic partners (either long or short-term). In 2012, the New York Times reported on a password-sharing trend among teenagers [108]. Teenagers sometimes choose to share the passwords for their Facebook or email accounts with their boyfriends and girlfriends as an expression of trust and love [108]. Boyd [21] speculates that this behaviour is learned from parents who insist on knowing their childrens' passwords. Adult partners also choose to share passwords. Singh et al. [121] examined how people manage money and banking, particularly in the context of relationships, and found that many couples share banking passwords. This sharing had to do with convenience, shared circumstances and trust. Although the practices broke terms-of-service requirements, the personal and social imperatives were seen as more important.

Another relationship that can affect password-sharing habits is the relationship between guardians and their dependents, whether parents and underage children, or between adult children and aging parents. These relationships are sometimes casual and based on trust and convenience, but they may also have legal significance, such as a power of attorney. Kaye [73] shares evidence that some parents insist on knowing their children's passwords, and Boyd [21] describes techniques that families use to moderate the trust relationship, such as having the child put their passwords in a piggy bank that the parent may break in the case of an emergency. Families may also share passwords for services that are used by all family members. Egelman, Brush and Inkpen [48] studied how families share computer user accounts, and found that some families used individual accounts while others shared a single account.

Changing contexts affect password sharing - there are situations in which a user may wish to begin sharing their passwords (e.g., in the case of personal injury, or end of life) or stop sharing their passwords (e.g., in the case of divorce). Locasto, Massimi, 
and dePasquale [87] describe issues that may affect the management of information at the end of life, including the sharing of passwords and account information. Google recently introduced their Inactive Account Manager which lets users set up an emergency contact for accounts that have been inactive for a given period of time. Other changing contexts that occur in the home are the decreases in parental responsibility for the information of children and teens. Parents and children may initially share passwords and account information, but eventually, the child will assume full responsibility for their own accounts. Joint custody of children after a divorce also brings changes to the technological landscape and may affect existing arrangements [99].

Culture may also affect the way that people choose to share their passwords. Hofstede [66] identified four major dimensions of cultural differences, which affect peoples' choices and interactions. Other more recent studies differ in their identification of dimensions, but the most consistent through all studies is the dimension that describes differences from individualism to collectivism [58]. The individualism/collectivism dimension describes the extent to which people place importance on belonging to groups (notably, family groups). Individualist societies place high importance on individual rights, while collectivist societies place larger emphasis on the interests of the group. Most computer infrastructure is designed in the United States, which has a highly individualistic culture [66], and the assumptions made regarding computer security likely reflect the values of the American individual. These values may differ from those of other users, particularly in the context of the home and family group (rather than the workplace). Most password studies have also focused on users in individualistic western cultures. Singh et al. [121] studied banking practices among aboriginal Australian users and reported that PIN-sharing within family groups was the norm. This was clearly tied to culture in statements such as "I know that key cards and the members should be confidential, but that's not [our] ... way" (cited by Singh et al. [121]).

\subsection{Addressing the Password Problem}

After acknowledging and better understanding the problems with passwords, it still remains to address those problems. Passwords have useful properties, and it seems 
possible that we may be able to fix the problems with passwords while still enjoying these benefits. The work highlighted in this section attempts to solve aspects of the password problem, whether by improving existing text password schemes, or through the development of novel authentication approaches.

\subsubsection{Alternative Text Password Schemes}

One method of addressing the password problem is to update the design of text passwords to promote memorability or security. A variety of modifications to traditional text password schemes have been proposed and studied.

\section{Passphrases}

Passphrases are a variation of passwords where the password consists of a string of words (a phrase) concatenated to form a password. Passphrases are often held up as a strategy that is both secure and usable [75]. Secure because the length of the passwords protects against brute force attacks, and the concatenation of words protects against dictionary attack; and usable because natural language words are used and common phrases are supposedly easily memorized.

Keith, Shao and Steinbart [74] conducted a twelve-week usability study of userselected passphrases. Their results showed that participants had many failed login attempts, which led to dissatisfaction with passphrases. They experimented with the design of passphrases, and concluded that passphrases could be better designed to support usability, including modifications for the user's typing speed and the semantic meaning of words in the phrase. However, they did not investigate how these design choices affected the security of the passphrase.

Shay et al. [117] conducted an extensive study of passphrases, experimenting with both the usability and security of several variations of passphrases. They studied eight types of system-assigned passphrases, and compared them to traditional passwords. Their results showed that most participants did not find the assigned passphrases at all memorable, and that they may even be less usable than ordinary randomly assigned text passwords because of the login times and increased numbers of error associated with the passphrases. 
While passphrases are often held up as more secure, they are also susceptible to dictionary attack. Kuo, Romanosky and Cranor [82] investigated the usability and security of mnemonic phrase-based passwords. These passwords were a variation of passphrases where users were instructed to create a password by thinking of a memorable sentence or phrase; selecting a letter, number, or special character from each word in the phrase; then collating the selected letters to form a password. The study collected instances of these kinds of passwords in a survey, and found that many of the passwords were derived from movies or books, suggesting that an attack dictionary could be easily compiled. Bonneau and Shutova [20] created a dictionary of phrases using proper nouns (such as band names and book titles), idioms, slang phrases and sports phrases and showed that users are more likely to select predictable and easily attacked passphrases. Their study indicated that even a four word passphrase may have less than 30 bits of entropy, which does not support the hypothesis that longer passphrases are particularly secure.

\section{Pronounceable Passwords}

Pronounceable passwords are random passwords that are designed to be easily pronounced. The intention is that these passwords will be easily remembered, but difficult to guess because they do not correspond to dictionary words. The first algorithms for generating such passwords were developed by Gasser [56], and pronounceable password generators were most prominently included in the Unix operating system. This work has been built upon by others $[55,22,144]$ and even leveraged for use in other areas (e.g., the generation of website domain names [37]).

Although the presumed advantage of pronounceable passwords over regular text passwords is increased memorability, little work on the memorability of such passwords exists. Shay et al. [117] compared pronounceable passwords with other assigned passwords and passphrases in a large scale study, and found that pronounceable passwords compared favourably with passphrases on login times and accuracy. Their study showed that when participants did not write their passwords down, pronounceable passwords had memorability advantages over passphrases. Leonhard and Venkatakrishnan [85] conducted a paper-based study comparing the memorability of pronounceable passwords with random text passwords and passphrases, but did not find conclusive results. Lau et al. [84] compared the memorability of pronounceable passwords with that of random passwords, and found no significant differences. 


\section{Persuasive Passwords}

Several projects have explored how persuasive techniques can be integrated into the design of text password systems to help users create and remember better passwords.

Forget, Chiasson, van Oorschot and Biddle developed Persuasive Text Passwords [54], a text password system that inserted a small number of random characters into userchosen passwords in order to increase security while maintaining the memorability of user-chosen passwords. However, their user study showed that participants would decrease the security of their user-chosen strings to compensate for the additional difficulty of remembering the inserted characters, meaning that no increase in security resulted from the persuasive mechanism.

Another technique for persuading users to choose good passwords is not to allow them to choose bad passwords. Proactive password checkers assess passwords at create time for a variety of characteristics, including the presence of various character sets, password structure, or password length [13]. Spafford [122] suggests checking passwords against a dictionary at create time to prevent users from choosing passwords easily guessed by dictionary attack. Schechter, Herley and Mitzenmacher [112] proposed a proactive password checker that assessed the popularity of passwords in an existing set, and prohibited users from choosing passwords that already existed in high frequency in the set. The aim of this work was to flatten the distribution of the effective password space, and protect passwords from statistical guessing attacks.

Bonneau and Schechter [19] were interested in increasing the memorability of assigned random passwords. They used spaced repetition, a memorization technique from the psychological literature that tests learning material at increasing time intervals, to encourage users to memorize random 56-bit text passwords. They found that $94 \%$ of their participants were able to remember their passwords, but a long and arduous training phase was required, with 90 logins over 15 days needed to teach users their passwords.

Komanduri, Shay, Cranor, Herley and Schechter [79] developed Telepathwords, a persuasive system that influenced text password choices by showing users predicted subsequent letters as they entered their passwords. The system predicted characters based on autocompletion algorithms, repeated strings, keyboard patterns, and interleaved strings, and discouraged users by making these predictions visible to users. A 
large scale evaluation showed that Telepathwords passwords were significantly more secure, while remaining as memorable as passwords chosen under other password policies. However, users expressed significant frustration with the password creation process for Telepathwords.

\subsubsection{Graphical Passwords}

Another approach to solving the password problem is to develop alternative authentication schemes that preserve the properties of passwords, but do not follow the traditional text format. One of these alternative approaches is graphical passwords, defined by Biddle, Chiasson, and van Oorschot [11] as "knowledge-based authentication mechanisms where users enter a shared secret as evidence of their identity, ... with the shared secret being related to or composed of images or sketches." (p.1) Much research has been conducted on graphical passwords, and they are beginning to see limited deployment, notably in the Android pattern unlock and the Windows 8 picture password.

Graphical passwords leverage the psychological finding that humans are able to better remember images than text [101]. They were developed in response to the password problem in the hope that image-based passwords could be both memorable and secure. A variety of graphical password schemes have been proposed, many of these with good usability and security properties. We mention notable examples of graphical password systems in Section 2.4.2, but do not attempt to survey the entire field.

Graphical passwords leverage the picture superiority effect [101], a psychological result that says that humans have better memory for images than words. The picture superiority effect is thought to be due to dual coding. Dual coding theory [100] speculates that the human brain encodes visual information in two forms: once in a visual form corresponding to the image, and once in a verbal descriptive form. This dual encoding makes the information more available upon retrieval, and thus leads to better remembering. More information about the picture superiority effect and the memory processes for passwords can be found below in Section 2.5.

An important feature of some graphical password schemes is that they support cueing. A cue is a signal to the user that reminds them of the password they are trying 
to remember, without disclosing any information about the password itself. The images in graphical passwords help users to better remember their passwords, and to distinguish their passwords from each other. They can also help minimize multiple password interference [30], where a user confuses two passwords, or matches a correct password to an incorrect account. Encoding specificity theory [133] finds that cues are only useful if they are present at the time that the memory is encoded. Thus, for passwords, the image cue must be present at password creation for it to be most useful at password entry. The cues provided in graphical passwords are visual, but cues may have other modalities, and are not limited to graphical passwords. Cued graphical password schemes include PassPoints [147], Persuasive Cued Click-points [32], and Image PassTiles [126].

\section{Example Graphical Password Systems}

A wide variety of graphical password systems have been proposed and studied, and a comprehensive survey of graphical passwords is available from from Biddle, Chiasson, and van Oorschot [11]. In this section, we highlight and explain example schemes that are relevant to this thesis.

Draw-A-Secret: Draw-A-Secret (DAS) [70] is a proposed graphical password system where a user creates a password by drawing an image on a grid (Figure 2.1). To log in, the user has to recreate the drawing, with pen strokes crossing grid boundaries in the same order, and pen-up and pen-down events happening in the same squares as in the original drawing. DAS was originally intended for use on Palm Pilot-type devices, but was not prototyped or tested by the original authors. Background Draw-A-Secret (BDAS) [45] is a variation of DAS that inserts a background image behind the grid. User studies showed that this modification encouraged users to choose more complex passwords.

PassPoints: PassPoints [147] is a graphical password system where the user is shown an image, and asked to choose five distinct click-points on that image. To log in, the user must click on those same five points in the same order. Since it would be difficult for users to click the exact pixel at every login, a tolerance region is built around each point, and any click within the tolerance region is accepted. Figure 2.2 shows the login screen for PassPoints. User studies of PassPoints [26] found that PassPoints 


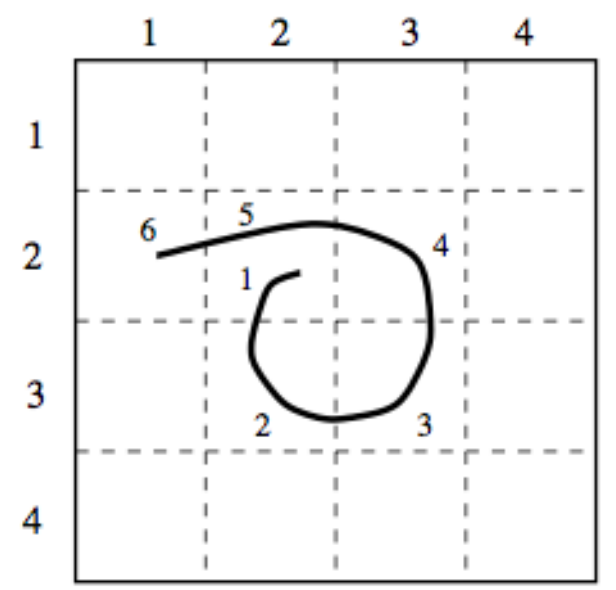

Figure 2.1: The Draw-A-Secret password grid, showing a sample password.

was very susceptible to hotspots, or areas of the image with an increased likelihood of password clicks, because different users often chose click-points in the same places.

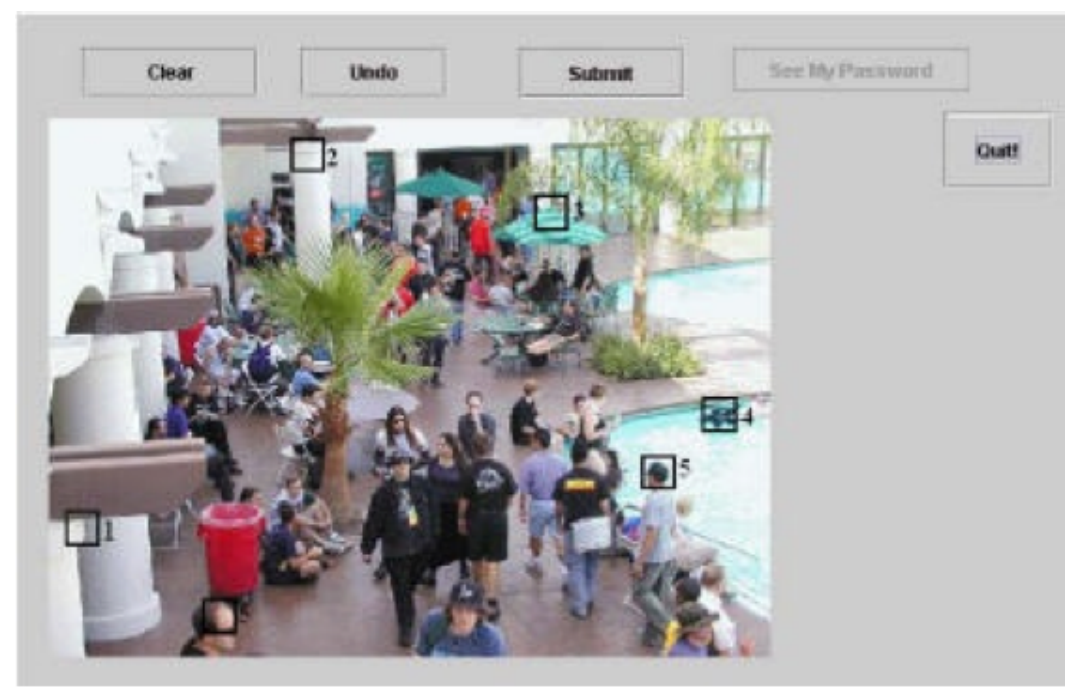

Figure 2.2: The PassPoints login screen. The numbered boxes show the tolerance regions around the user's ordered click-points.

Cued Click-Points \& Persuasive Cued Click-Points: Cued Click-Points (CCP) [34] works similarly to PassPoints, but instead of choosing five click-points on one image, the user is asked to choose one click-point on each of five images. When logging in, the user must click again on the correct point on each image. The images are presented in sequence, and feedback is built into the system - if the user clicks on an incorrect point, they see an incorrect subsequent image, immediately alerting the user to their error. Persuasive Cued Click-Points (PCCP) [32] is a close variation of 


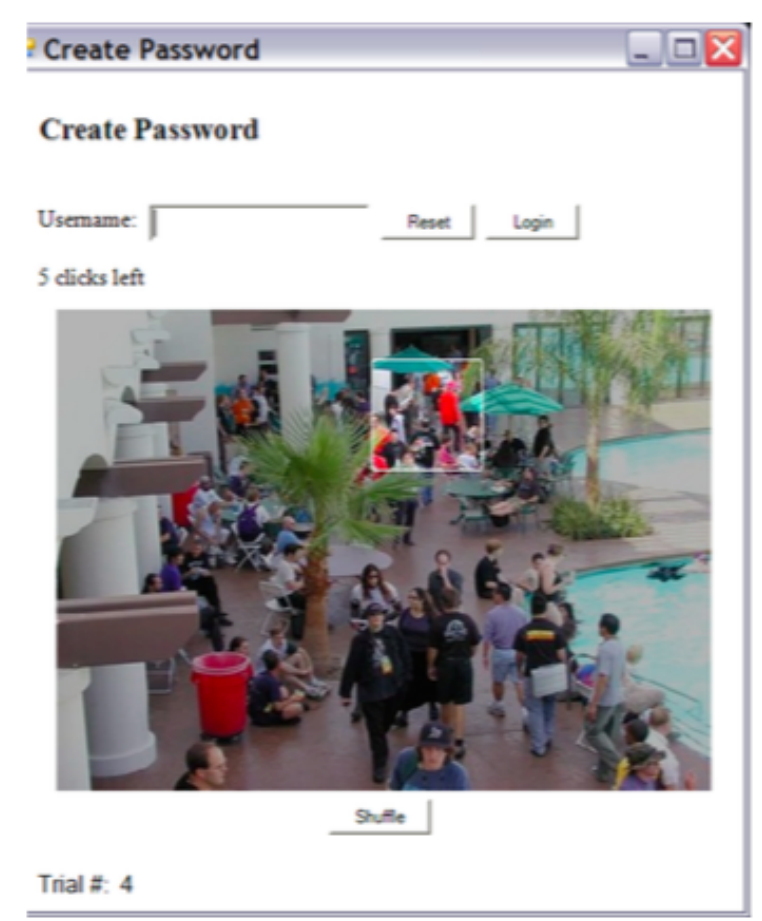

Figure 2.3: The password creation screen for PCCP. The user must pick their clickpoint within the highlighted box. The box is arbitrarily placed on the image, and its location can be randomly moved by clicking the "shuffle" button.

$\mathrm{CCP}$ that uses a persuasive mechanism during password creation to discourage users from choosing their click-points on hotspots. Figure 2.3 shows the password creation screen for PCCP.

PassFaces: Passfaces [105] is a commercial graphical password system that assigns each user a set of three faces. To log in, users are shown an array of nine faces (shown in Figure 2.4), and must choose the face that is part of their assigned password. For added security, users must choose the correct face from three arrays, and the distractor faces are always the same.

\section{Types of Graphical Passwords}

Graphical passwords can be categorized by distinguishing the type of input requested by the scheme [42]:

Drawmetric: These systems ask the user to reproduce a drawing on a grid. Examples include Draw-a-Secret (DAS) [70], and Background Draw-a-Secret (BDAS) [45]. 


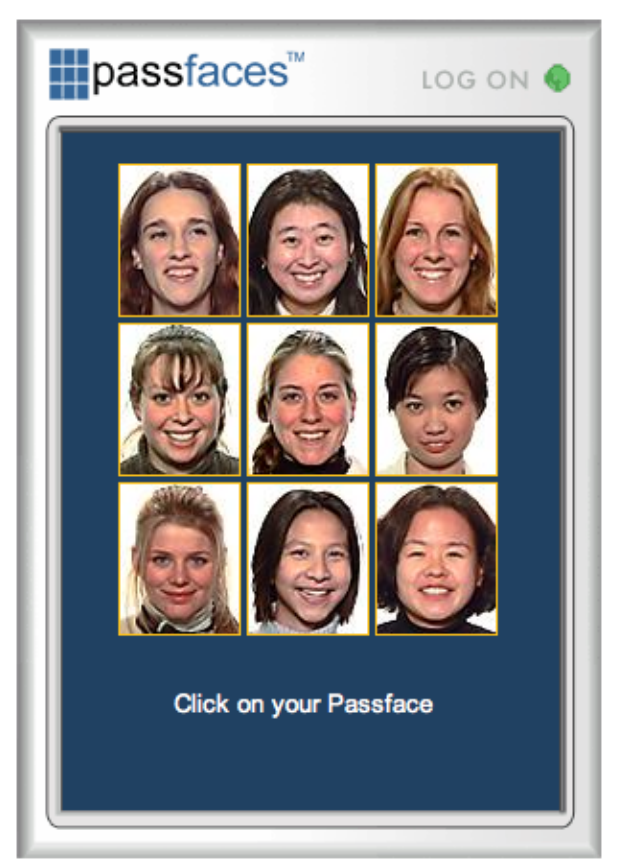

Figure 2.4: The Passfaces login screen, showing an array of nine faces from which the user must select the one that is part of their password.

Locimetric: In these passwords, the user is asked to accurately click on points on an image. Example schemes include PassPoints [147], and Persuasive Cued ClickPoints [28].

Cognometric: These schemes ask the user to recognize and identify images belonging to their set of password images from a set of distractor images. An example scheme is PassFaces [105].

Most drawmetric graphical password systems leverage the picture superiority effect by using a grid-drawing exercise, but there is no reason that the type of login task will affect the type of memory retrieval that is leveraged. When no cue is given in a graphical password system, the password system will leverage free recall. Different locimetric graphical password systems use different methods of password entry, but all locimetric graphical password systems provide users with visual cues to help them more easily recall and distinguish their passwords, thus leveraging cued recall. Cognometric graphical passwords work by presenting a grid of images, where one image belongs to a known set of "password" images, and the other images are distractors, and the user must correctly choose the password image to authenticate. They leverage recognition memory by explicitly displaying all possible choices to the user, and 
expecting the user to recognize the correct option. It is also important to note that the mapping of input type to retrieval type may not always align this way: BDAS is categorized as drawmetric because it asks the user to draw a figure to log in, but it leverages cued recall memory by providing the user with a distinct background image.

Most graphical password systems allow users to select their own passwords. However, work on user choice in graphical passwords $[41,29,136,137]$ has shown that users tend to choose predictable passwords that can be exploited in a dictionary attack. Assigning random passwords protects against this kind of attack. In some cases, the predictability of user-chosen passwords can significantly affect the security of the system. Davis, Monrose, and Reiter [41] showed that users tended to choose Passfaces passwords with strong gender and attractiveness biases, and these biases opened the passwords to attack. Following these results, Passfaces stopped allowing users to select their own passwords and implemented assigned random passwords.

Assigning passwords presents different challenges for the different types of graphical password. The complexity and granularity of existing drawmetric password systems makes assigning passwords difficult. Clearly conveying the appropriate details is problematic in systems where there are many subtle nuances to password entry that can be difficult to convey to users. Existing locimetric schemes such as PassPoints [147] require precise input that can create difficulties in communicating assigned passwords to users. However, there is no reason that a visual cue could not be used in a password system that required less precise input. Cognometric passwords are easily assigned by showing the user their set of password images at password creation.

\subsubsection{Password Managers}

Another approach to solving the password problem is not to change passwords themselves, but to design strategies to handle the problems with passwords for users. Password managers are programs that store and enter users' passwords, thus saving the user from remembering their passwords or which passwords are associated with which accounts. Password managers are one of the few solutions to the password problem that have seen extensive deployment. A variety of password managers are commercially available, but it is not clear how widely they are used.

There are two models for password managers [33]: they either generate a password 
at login by hashing the user's master password together with information from the website, or they store the user's passwords in a password "wallet" which is protected by a master password (which may be required at every website login). In practice, most modern password managers adopt a variation of the wallet model, where a list of passwords is securely stored.

Examples of dedicated password managers include LastPass [83], KeePass [106], Roboform [119], myllogin [93], Password Box [103], and 1Password [2]. Some of these password managers (such as LastPass) are available in multiple formats, including browser extensions, mobile applications and online; others (such as PasswordBox) are entirely online. Most password managers autofill passwords on websites, either through a browser extension that the user must install, or via a bookmarklet saved in the browser's bookmarks. A bookmarklet is a short piece of JavaScript that is saved as a bookmark and can be executed when the bookmark is opened.

Password managers save existing account passwords, but dedicated password managers usually have the ability to suggest passwords for the user, aiding them in the creation of secure, different passwords for each account. Some password managers include additional features to help users understand their web security. LastPass contains an audit feature that identifies weak and reused passwords, and suggests ways for users to improve their security [83].

Password managers are also included in most web browsers, including Mozilla Firefox, Chrome, Internet Explorer, and Safari. These password managers are not usually as fully-featured as dedicated password managers, and only save and fill existing passwords. Browser-based password managers save passwords when they are typed into the appropriate fields, and then automatically input them when the page is visited again. They may be configured with a master password, or may simply fill saved passwords when a site is visited.

Although password managers seem to be one of the best existing technologies for helping users address the password problem, research on the security of password managers has exposed a number of vulnerabilities.

The fundamental vulnerability of password managers is that they provide a single point of failure where an attacker may potentially discover all of a user's passwords. If the master password protecting the password manager is predictable or weak, a 
user may lose all of their passwords in a single attack. Some password managers (e.g., most browser-based managers) even allow the master password to be turned off, allowing any user to gain access to any account stored by the password manager.

Web-based password managers allow users to access passwords from multiple computers or devices, giving obvious usability benefits. However, a security analysis of web-based password managers [86] showed that these managers need to be carefully implemented to avoid security vulnerabilities, including bookmarklet vulnerabilities, web vulnerabilities (cross-site scripting and cross-site request forgery attacks), authorization vulnerabilities, and user interface vulnerabilities.

The autofill features of password managers can be exploited in a variety of ways to expose passwords. Silver et al. [120] examined ten password managers and found that the managers varied widely in how autofill was implemented and managed. They discuss a number of corner cases where policy decisions can affect where passwords are autofilled. These corner cases include changes of protocol (e.g., HTTPS to HTTP), modified form actions (e.g., post vs. get), modified password field names, and changes to the domain and path of login pages. They also showed that passwords can be remotely extracted from password managers using sweep attacks implemented via malicious routers and iFrames or HTTP redirects.

Less research has investigated the usability of password managers. Chiasson, Biddle and van Oorschot [33] studied the usability of two password managers (one walletbased manager and one hashing manager) and found that both managers had significant usability issues. Worse, participants had poor mental models for how the software worked, and these poor mental models led users to make dangerous and unrecoverable security errors. The usability issues identified in the study affected the ability of password managers to securely protect users' passwords.

Another significant usability issue for password managers is related to trust. It can be unclear to users who has access to passwords stored in password managers, and many users are concerned about entrusting passwords to a an online password manager. A usability study of three password managers showed that users prefer to manage their passwords in a location controlled by them, such as a mobile phone, rather than having them managed by a third-party in an unknown storage location [72]. Although not well-studied, it is possible that the low adoption of password managers is because of trust-related issues. 


\subsubsection{Single Sign-on}

Single sign-on (SSO) systems are services that try to solve the password problem by having a single entity provide authentication for multiple websites. Users authenticate to an SSO provider, who checks their credentials and relays the result back to the website. A variety of architectures for SSO exist [102]. Two SSO standards are OAuth and OpenID, and well-known SSO providers include Facebook and Google.

SSO has not seen wide adoption, either by users or websites [129, 130]. Sun, Boshmaf, Hawkey and Beznosov identified this lack of adoption as due to misaligned incentives between users, identity providers, and relying parties [129]. A study of OpenID [130] showed that users had a variety of concerns with SSO, including privacy and trust. The study also found that users misunderstood how SSO worked, and that it did not fit their existing habits.

\subsubsection{Challenge Questions}

Another password alternative is to allow access to accounts through the use of challenge questions (also referred to as "personal validation questions" or "secret questions"). Most familiarly seen as part of the password reset process, challenge questions are asked by the system and the user must provide the correct response [71]. The idea is that a user will be able to remember the correct answers without rehearsal, but that another person would not be able to easily guess a user's responses.

Zviran and Haga [151] introduced the idea of passwords cued by personal information. They tested the memorability of these "cognitive passwords", and investigated how easily guessed they were by the user's significant other. They asked participants to answer 20 cognitive password challenge questions, and found that average participant remembered $78 \%$ of their items after three months. However, when they asked someone close to the user to answer the same questions, the significant other was able to correctly answer $40 \%$ of the questions.

Challenge questions have been shown to have a number of problems: they typically have small password spaces, making them susceptible to statistical guessing attacks [17], and they have been shown to be vulnerable to personal attacks, where 
an acquaintance has enough knowledge to guess the responses [111]. Users also sometimes find it difficult to remember their responses to these questions. Questions such as "What is your favourite colour?" have a limited answer space, and users may not always have a strong and continuous preference.

Challenge questions are similar to cued passwords because the question provides a cue to the user to help them remember a response. This cue is usually text-based, but Renaud and Just [107] proposed using random image cues for challenge questions. They found that the image-based challenges were more memorable, while maintaining equivalent security.

\subsection{Memory Theory for Passwords}

In this section we highlight memory research from the psychological literature that may relate to various kinds of knowledge-based authentication.

\subsubsection{Picture Superiority Effect}

Graphical passwords leverage the picture superiority effect [101], which is the finding that people have better memory for images than words. The picture superiority effect is seen in tests of both recall and recognition.

Paivio's dual coding theory [100] theorizes that the brain has separate mechanisms for remembering image-based information (such as objects, images and events) and for remembering verbal information (both spoken and written). The explanation for the picture superiority effect is that dual coding occurs when people remember images. The images are encoded visually and are also encoded in a verbal form (as in a description) and remembered semantically.

The distinctiveness account [91] provides an alternate explanation for the picture superiority effect, and the theories in this account hypothesize that images have implicitly memorable properties. The sensory-semantic model [95] argues that the picture superiority effect occurs because images have distinct sensory codes allowing them to be more easily accessed in memory (although words and images have identical 
semantic encoding). This theory is supported by evidence that visual similarity in images leads to decreases in the picture superiority effect [96].

Another theory is the levels-of-processing theory [36]. This theory proposes that the quantity and quality of processing affects the endurance of information in memory. Nelson and Reed [94] applied the levels-of-processing theory and found evidence that the picture superiority effect stems from the different processing that takes place for images and words.

The picture superiority effect is not particularly robust, and can be disrupted or eliminated by a variety of factors. The use of pictures with similar schematic appearances can minimize the effect [96], and a rapid presentation of images can also disrupt the effect [95]. The nature of the retrieval task can also minimize or even reverse the effect [142].

\subsubsection{Memory Retrieval}

Different password schemes leverage different types of memory retrieval through their design. The differing kinds of retrieval affect not only memorability, but other factors, such as the time to login, or the ease of use.

Recall and recognition are processes of retrieving information from memory. Framed in early work as opposite memory tasks, recall is the process of remembering a specific focus when the context is provided, whereas recognition is the process of remembering the contextual information when the focus is provided [67]. Recall can be divided into cued recall, where a cue provides assistance in retrieval of the correct memory, and free recall, where no support is given. Recognition is almost always found to be superior to recall [62], and there are several theories that attempt to explain the differences.

The generate-recognize theory [6] frames retrieval as a two-step process, consisting of both a generation and a recognition phase. If, for example, a password is retrieved from memory, long term memory is searched for a list of candidate passwords in the generation phase. Then each of the words in the list is evaluated to see if it can be recognized as the looked-for password in the recognition phase. 
Some of the differences between recall and recognition can be explained by the generate-recognize theory [140]. Recognition memory uses only the recognition phase (and does not need the generation phase), so it is faster and easier to perform. The benefits of cueing on memory retrieval are also explained by the theory. Cues are used to help generate relevant candidate list for recognition, and also help the person recognize the appropriate word from that list. There are also findings that contradict or are not explained by the generate-recognize model. Consistent cues are most effective, and when cues are changed (e.g., "sail" vs. "gravy" for boat), participants have more difficulty recalling the appropriate word [140]. However, the generate-recognize theory would predict that all cues work equally well.

Encoding specificity theory [133] was developed to explain the effects of unlearned cues on recall memory. This theory builds on levels-of-processing theory, and states that only information processed at the time of storage can later be used as retrieval cues. If semantic information about a word is processed at the time of learning, then that information can successfully be used to cue memory. Thus, the word "table" can only be used to cue memory of the word "chair" if the subject encodes the semantic information linking the two objects at the time of encoding. According to the encoding specificity theory, if the word "orange" is encoded in the context of a fruit name, it will not be successfully cued with the suggestion of a colour name.

The underlying question in the literature about memory retrieval is about the nature of the retrieval process. The continuity hypothesis sees recall and recognition as extremities on a continuum involving a single retrieval process. This counters the discontinuity hypothesis, which poses recall and recognition as separate retrieval processes. Different theories support different hypotheses: generate-recognize theory assumes that memory involves two processes, and that retrieval is therefore a fundamentally more complex process than recognition because it uses both processes. Encoding specificity theory assumes that retrieval is a straightforward process, and that the complexity happens at the time of encoding. Studies of different cues find support for the continuity hypothesis [134]. Although we refer to recall and recognition as if they are distinct processes, it is important to remember that they are end points on a wide spectrum of retrieval scenarios that integrate aspects of both.

The literature shows that recognition memory is nearly always better than recall memory [62], but some studies [132, 139] have shown that recall can sometimes be 
superior to recognition. Modifying contextual factors for how participants are asked to remember paired words affected the memorability of the word pairs. Pairs that formed compound words, such as "air / port" or "home / stead", were more memorable via recall than recognition.

\subsubsection{Memory Retrieval and Graphical Passwords}

In our earlier work [124], we conducted preliminary studies investigating how the different kinds of memory retrieval affect the memorability of randomly assigned graphical passwords. The results of these first studies suggested numerous follow-up studies, which are presented as part of this thesis. For clarity and context, we present a brief overview of the work here, but we emphasize that the results of this study are not intended to be taken as contributions to this thesis. We present a summary of the work here, and a more detailed write-up is found in Appendix A.

To study how the different types of memory retrieval affect the memorability and usability of assigned random graphical passwords, we conducted a week-long user study. The study had a between-subjects design, meaning that participants were assigned to a study condition where they used only one type of password system. There were five conditions: a free recall graphical password condition, a cued recall graphical password condition, a recognition graphical password condition, and we compared them to assigned text passwords and user-chosen text passwords. We used the PassTiles graphical password system to be able to easily compare users' performance. The text conditions were designed to have equal password space to the graphical password conditions and used a standard text password system.

In the study, participants created three passwords in the lab, and were asked to log in from home over the following week. We had two measures of memorability: the total time that users were able to remember their passwords, and the number of password resets. We assessed usability by measuring the length of time that it took users to log in. Our hypotheses were that recognition-based graphical passwords would be more memorable than both other kinds of graphical passwords and text passwords. We

also hypothesized that there would be differences in login time among the five study conditions. 
We found clear differences in usability between the study conditions. Participants took significantly longer to log in using the recognition-based passwords than any of the other password systems. Although participants were able to easily remember recognition-based passwords, it took them almost 30 seconds on average to log in.

The results for memorability were somewhat inconclusive. We found that participants in all conditions were able to remember their passwords for the duration of the study, and we did not see any significant differences between conditions. Similarly, very few participants (in any condition) reset their passwords, leading to a lack of significant differences. We were surprised by this ceiling effect: we had expected that it would be difficult for users to remember three passwords over one week and had expected to see more memory failures. We thought that perhaps we had made the study task too easy, and that conducting the test with stronger passwords might show more differences. The post-test questionnaire also revealed that many participants had written their passwords down, and post-hoc, we concluded that this artificial aid had obscured differences in memorability between the study conditions.

In the follow-up studies presented in Chapter 4, we conducted three additional studies to investigate how memory retrieval affects the memorability and usability of graphical passwords.

\subsection{Summary}

Designing secure and memorable authentication is a difficult task, and the fundamental problem with text passwords is that passwords that are easy for users to remember are typically also for attackers easy to guess. Although problems have been identified with text passwords, there is yet to be a widely adopted solution.

Research on users and their passwords have shown that users have many accounts [50], and have difficulty choosing secure passwords for those accounts. Many users turn to strategies such as reusing passwords or writing them down to help with remembering. Circumstantial factors, such as a need to share passwords, may also affect users' password habits. Although these are usability problems, they have a direct and negative impact on the security of accounts. 
There has been much work addressing the password problem. Approaches include alternative text password schemes, graphical passwords, password management software, single sign-on systems, and challenge questions. Some of these are available commercially, or implemented for use by end users. However, none have seen widespread adoption, and passwords continue to be a problem for many users.

In this thesis, we take a practical approach to solving the password problem. One solution to the password problem would be the creation of strong, memorable passwords. Existing passwords have privacy and revocation advantages, as well as substantial existing infrastructure, and it would be advantageous to retain these characteristics. Graphical passwords offer possibilities for drop-in replacement: they combine many of the advantages of text passwords with increased memorability, and we explore this possibility in Chapters 3 and 4.

However, users have many passwords to manage, and keeping track of and remembering large numbers of passwords presents additional difficulties. In Chapters 5, 6, and 7 , we explore users' experiences with multiple passwords and the coping strategies that they use to manage these large sets of passwords and accounts. To combine our findings about password memorability and users' coping strategies, we propose and present a working prototype of a new password manager in Chapter 8. This password manage supports password reuse and allows graphical passwords to be used for login to any website. 


\section{Chapter 3}

\section{PassTiles}

\subsection{Introduction}

The best way to ensure protection against guessing attacks is to assign randomly chosen passwords. Since randomly assigned passwords are all equally probable, attackers are left to systematically guess each password, rather than being able to prioritize probable passwords. However, randomly assigned passwords can be difficult for users to remember, and users often strongly dislike such passwords because they are difficult to learn and recall.

Most graphical password systems have relied on user-chosen passwords, but research has shown that users are still likely to choose predictable passwords [137]. Studies of the commercial system Passfaces showed that users were far more likely to choose attractive female faces in their passwords, and that this resulted in vulnerability to dictionary attacks [41]. In response to this research, the designers of the Passfaces implemented system-assigned passwords. Other attempts to mitigate the effects of user-chosen hotspots have included persuasive approaches where the system gently persuades the user to select a more random password [54, 32, 19].

Little work has addressed the issue of making randomly assigned passwords more usable. Bonneau and Schechter [19] used spaced repetition to persuade users to memorize 56-bit random text passwords, but their approach had usability disadvantages: the memorization process took 15 days and 90 rehearsals. 


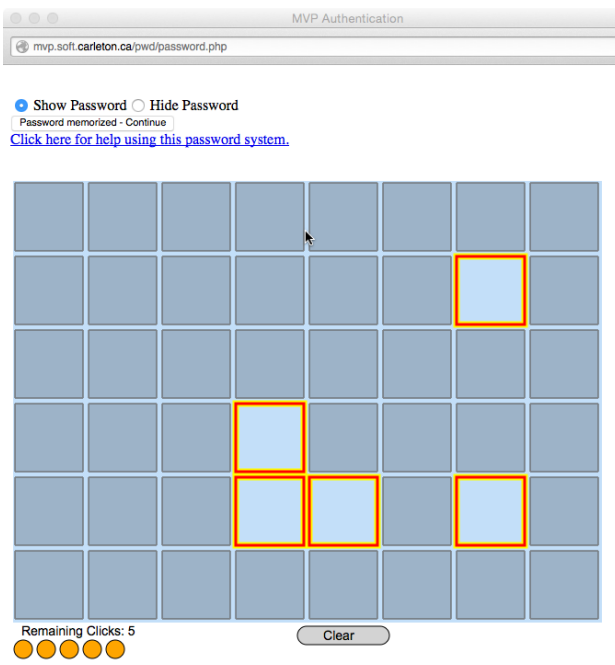

Figure 3.1: The Blank PassTiles password creation screen, showing a $6 \times 8$ grid and an assigned five tile password.

We speculate that the usability advantages of graphical passwords should be equally applicable to randomly assigned graphical passwords. Many studies on the picture superiority effect $[101,96,94]$ and memory retrieval $[132,6]$ were done with random stimuli, indicating that the effect should hold for randomly assigned passwords.

Many existing graphical passwords systems are designed in a way that makes assigning passwords difficult. To assign passwords, they must be easy to clearly communicate to the user, and there cannot be hidden steps or password components. Systems such as Draw-a-Secret [70] involve hidden components in the places that pen-up and pendown motions happen, as well as in the exact orderings of pen strokes. The granularity of click-based systems such as PassPoints [147] presents difficulties for password assignment because it can be difficult to communicate the exact click-point to the user (and especially difficult for them to remember that point if it is indistinguishable from its neighbours).

PassTiles (Figure 3.1) is a graphical password system that we originally designed for use in the memory retrieval studies described in Chapter 4. Our initial goal was to design a system to be able to compare different types of memory retrieval within the same password system, and to be able to easily assign passwords. Since we wanted to compare the effects of different types of memory retrieval on the memorability of graphical passwords, it was important to us that other features of the system stayed constant. 
The original variations on PassTiles were designed for work completed in my MA, and are not intended to be claimed as part of this PhD. However, we have since realized that PassTiles has a number of usability and security advantages in its own right, and deserves acknowledgement as a usable graphical password system. For the $\mathrm{PhD}$, we have expanded the PassTiles system to include more functionality, and examined its advantages apart from its original purpose. PassTiles is easy to learn, allows simple password assignment, and is free of the discretization issues affecting other cued recall graphical password schemes [12, 31, 149] (discussed below in Section 3.5.1). PassTiles is also highly configurable, allowing a variety of options (including security level) to be customized.

\subsection{Original PassTiles}

In our original studies, we created three versions of PassTiles. In all three, the user is presented with a grid of password tiles and their password consists of a subset of those password tiles. To log in, the user must click on the correct password tiles. In the standard configuration, an image is presented with a $6 \times 8$ grid, and passwords consist of 5 tiles. The order of tile entry is not significant. This gives a theoretical password space of

$$
S_{T}=\log _{2}\left(\begin{array}{c}
\text { width } \times \text { height } \\
\text { password length }
\end{array}\right)=\log _{2}\left(\begin{array}{c}
6 \times 8 \\
5
\end{array}\right)=21 \text { bits }
$$

Since we were interested in distinguishing the three different types of memory retrieval (recall, cued recall, and recognition), the three variations differed in the kind of image cue that they offered the user. If the system is configured with a plain or blank background to the grid, we refer to the system as Blank PassTiles (Figure 3.1). The blank background makes password retrieval a free recall task for the user. The patterns formed by the password tiles are similar to a mosaic and leverage visual memory similarly.

The grid background image may also be a single image, with the tile grid superimposed over the picture. We refer to this system as Image PassTiles (Figure 3.4). It leverages cued recall memory, and allows the user to remember their password in relation to the background image. Additionally, the background image serves as a cue to the password, helping users remember and distinguish multiple passwords. 


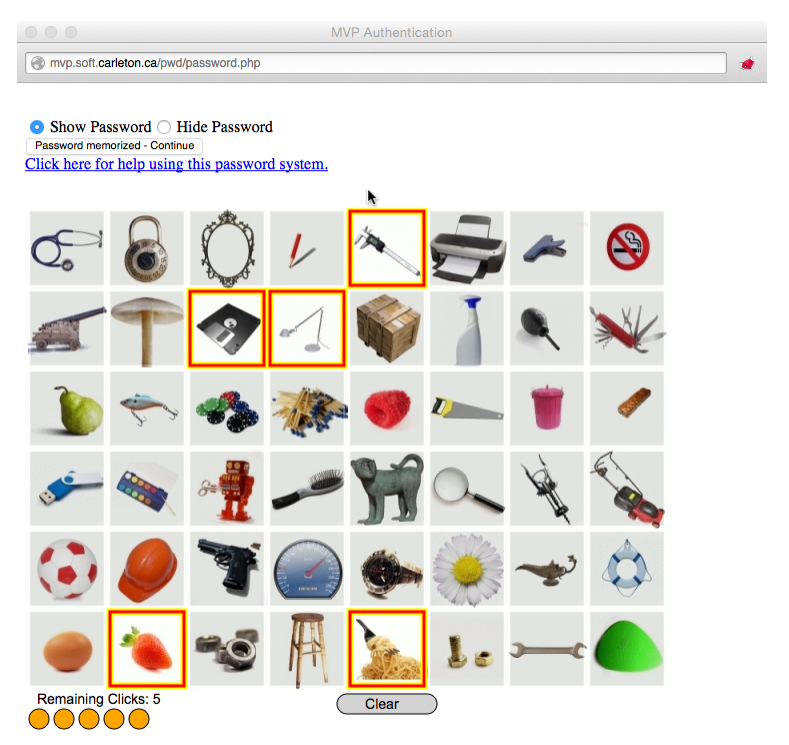

Figure 3.2: Password creation interface for Object PassTiles.

Instead of having a single background image, Object PassTiles (Figure 3.2) shows a unique object image inside each password tile, creating a grid of smaller object images. The password consists of a set of objects. The user must click on the correct set of objects (rather than grid squares) to log in. The grid of objects is shuffled at every login, so that the user's objects appear in different password tiles at every login. This forces users of Object PassTiles to rely on recognition memory, because the password objects are always shown on the screen, but must be recognized from the shuffled grid of items.

\subsection{Further Modifications}

In its original form, the three versions of PassTiles changed the background image(s) to affect the type of memory retrieval. However, there are a number of other ways in which PassTiles can be modified to improve its usability. In later studies conducted for my $\mathrm{PhD}$, we explored the usability of some of these modifications. We also developed a number of other modifications that can be used to customize PassTiles to more use cases, and we believe that this feature set gives PassTiles viability as a real scheme. Table 3.1 gives a list of the available features of PassTiles, and they are also discussed below. Note that most features can be combined with other features, with exceptions noted in the table. 
Table 3.1: Customizable features of PassTiles.

\begin{tabular}{|c|c|c|}
\hline Feature & Description & Notes \\
\hline Image Size & $\begin{array}{l}\text { Images may be any dimensions } \\
\text { demanded by screen space. }\end{array}$ & $\begin{array}{l}\text { Has no effect on pass- } \\
\text { word space. }\end{array}$ \\
\hline Grid Dimensions & $\begin{array}{l}x \text { and } y \text {-dimensions are indepen- } \\
\text { dently adjustable. }\end{array}$ & \\
\hline Password Length & $\begin{array}{l}\text { Can be set to any length up to the } \\
\text { number of grid tiles. }\end{array}$ & \\
\hline Background Image & $\begin{array}{l}\text { Allows the selection of a back- } \\
\text { ground image (default is blank). }\end{array}$ & \\
\hline Object Recognition & $\begin{array}{l}\text { Fills each grid tile with an indi- } \\
\text { vidual object image. }\end{array}$ & \\
\hline Ordered & $\begin{array}{l}\text { Boolean option determining } \\
\text { whether or not password must } \\
\text { always be entered in the correct } \\
\text { order. }\end{array}$ & \\
\hline Shuffle & $\begin{array}{l}\text { Boolean option determining } \\
\text { whether the object grid is } \\
\text { shuffled at every login. }\end{array}$ & $\begin{array}{l}\text { Only usable with Ob- } \\
\text { ject PassTiles. }\end{array}$ \\
\hline Shuffle Button & Optional shuffle button. & $\begin{array}{l}\text { Only usable with Ob- } \\
\text { ject PassTiles }\end{array}$ \\
\hline Digit Login & $\begin{array}{l}\text { Places a random digit in each } \\
\text { password tile so that the user } \\
\text { can enter their password via key- } \\
\text { board. }\end{array}$ & $\begin{array}{l}\text { Limits the theoreti- } \\
\text { cal password space if } \\
\text { there are more than } 9 \\
\text { password tiles. }\end{array}$ \\
\hline $\begin{array}{l}\text { User-chosen Back- } \\
\text { ground Image }\end{array}$ & $\begin{array}{l}\text { Lets the user choose their own } \\
\text { background image (from a prede- } \\
\text { fined set). }\end{array}$ & \\
\hline $\begin{array}{l}\text { User-selected Pass- } \\
\text { words }\end{array}$ & $\begin{array}{l}\text { Lets users choose their own pass- } \\
\text { words by selecting the tiles. }\end{array}$ & \\
\hline
\end{tabular}




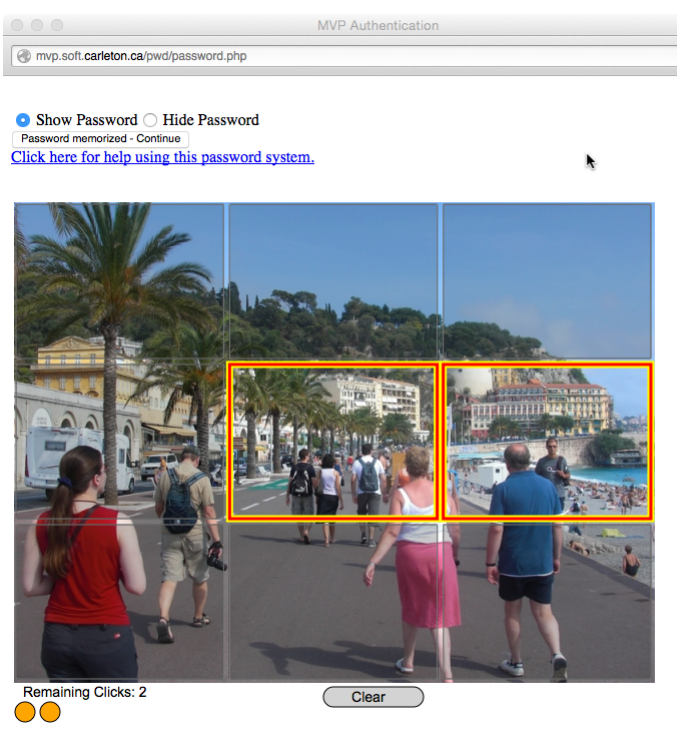

(a) $2 \times 3$ grid, with a two tile password.
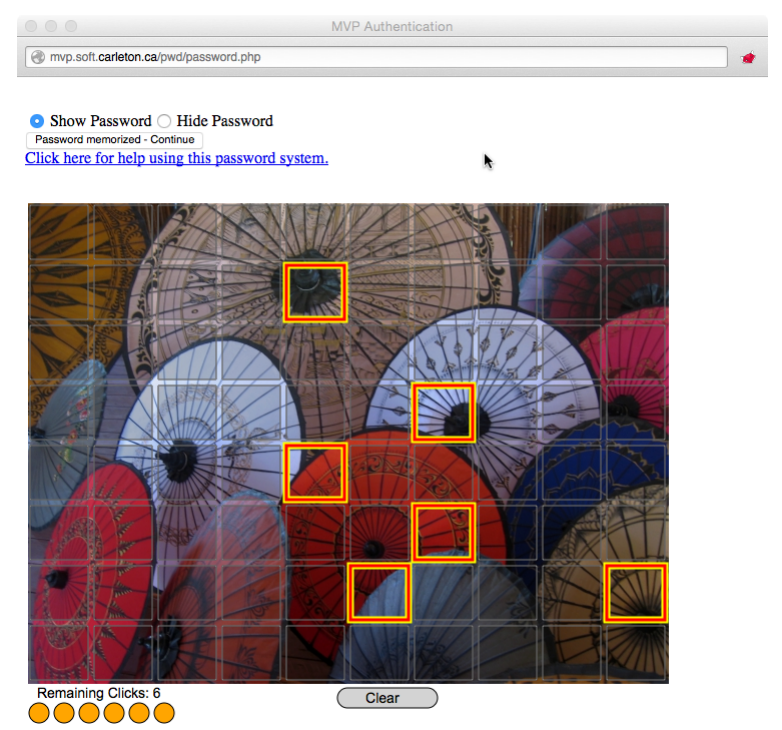

(b) $8 \times 10$ grid, with a six tile password.

Figure 3.3: Password creation interfaces for two different grid and password length configurations of Image PassTiles.

For the initial studies, we set the security of the schemes to 21 bits because of Florencio and Herley's suggestion [51] that 20 bit passwords should be strong enough to defend against online attack. However, the parameters that determine PassTiles' security are now all configurable. The grid dimensions and password length (number of tiles in the password) can be independently adjusted (Figure 3.3) to affect the size of theoretical password space. Password entry can be either ordered or unordered, which also affects the size of the password space (ordered password entry increases it).

Object PassTiles shuffled the images at every presentation to force users to depend on recognition memory, but the argument for shuffling as a design feature (as in PassFaces [105]) is that it defends against the threat of shoulder-surfing attacks and makes it harder for an attacker to simply memorize the positions of the grid squares. However, shuffling increases the difficulty of logging in even for legitimate users, and may not defend against more sophisticated recording attacks [77]. In our later modifications, we designed an optional shuffle feature. A shuffle button is always present, but the image tiles do not automatically shuffle at every login. When the user perceives a shoulder surfing threat to be present, they can press the button to discourage an attacker. When shuffling is disabled, users are able to rely on recall to remember the positions of the password tiles, which is an advantage to usability. 

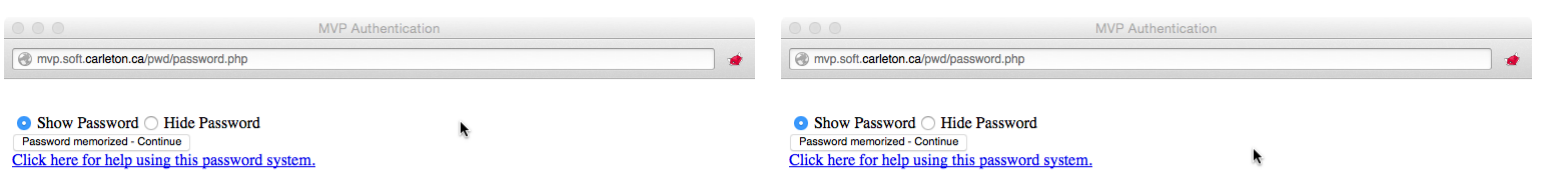

Click here for help using this password system.
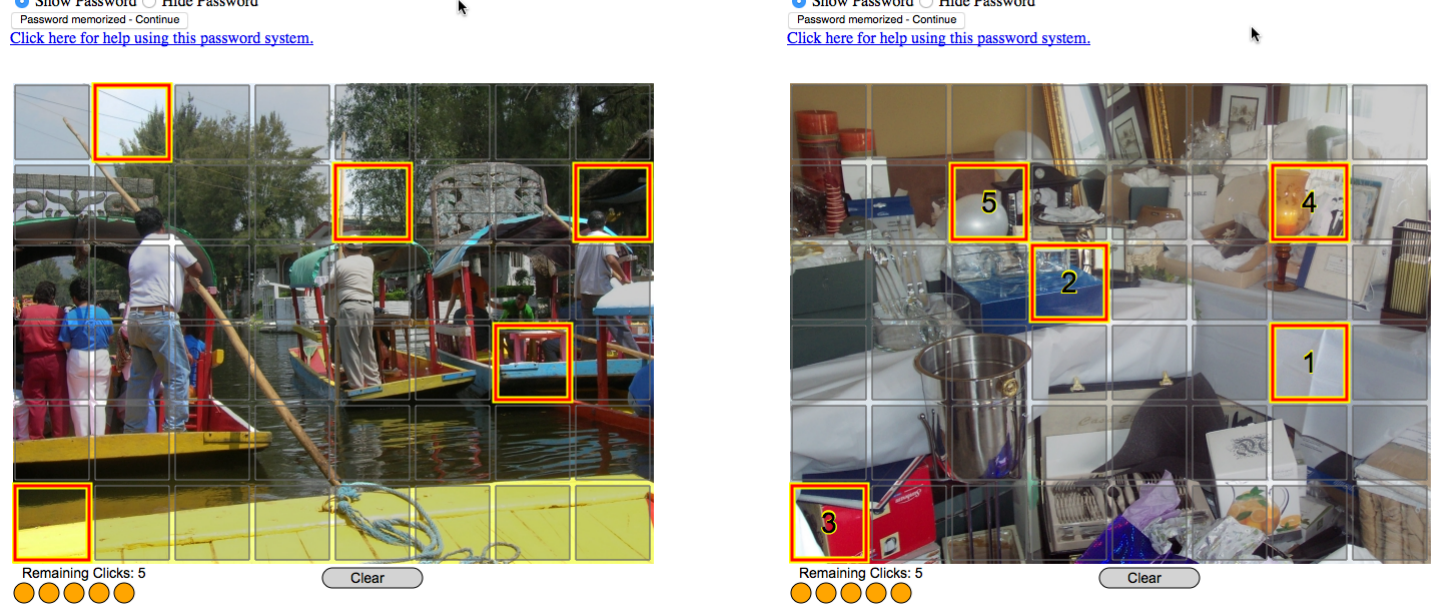

(a) Image PassTiles with an assigned random password.

(b) Image PassTiles with an assigned random password and ordered login.

Figure 3.4: Password creation interfaces for two variations of Image PassTiles.

Although we designed the system to facilitate assigned passwords, PassTiles may be configured to allow users to select their own passwords, and users may also be allowed to select their own background image (from a pre-defined set). The different PassTiles configuration options can also be combined to suit specific use cases. For example, one configuration option is to let the user select the background image for a randomly assigned password. If this option is chosen, the user chooses their image before seeing the password. This process avoids the formation of hotspots.

Another feature of PassTiles is the "digit login" option. In this configuration, the user does not click on the square to enter the password, but instead types a sequence of digits corresponding to their password. At every login, a randomly generated digit is shown in each grid square, and the user has to type the digits from their password squares. This PassTiles variation makes it similar to GrIDsure [60], a now-defunct commercial graphical password system that used grid-based passwords and digit login. Figure 3.5 shows the creation process with digit login enabled. 


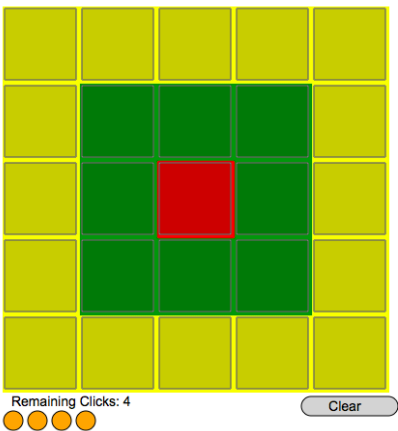

(a) The user first chooses their password by clicking on their chosen grid squares.

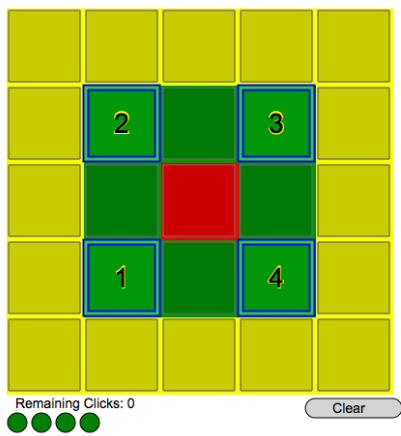

(b) The users' four clicks are recorded on the grid.

Figure 3.5: Password creation for a user-selected, digit login PassTiles password.

\subsection{Usability}

When designing PassTiles, one of our primary goals was to facilitate the memorability of assigned random passwords. Although it is tempting to view assigning passwords as analogous to users selecting passwords, assigning passwords actually has different usability requirements that require specific design. We were interested in multiple aspects of usability, including learnability and memorability. Learnability was important for our initial studies, because we wanted users to understand the system without much training, but it is also very important to the scheme's viability in real world use. The memorability of any password scheme is important because the secret must be memorable in order to be useful.

In a typical text password creation, the user creates and confirms their password by typing it twice: once to establish the password, and once to confirm that they typed it correctly the first time. However, this process makes less sense for assigned passwords, where the purpose of the confirmation step is to check that the user has memorized their assigned password, not just that they have typed it correctly. To help users memorize their passwords, we designed and included a practice phase for the password creation process in PassTiles. In this phase, the user can practice entering their assigned PassTiles password as many times as they need to memorize it. This takes advantage of memory rehearsal, the process by which information is transferred from short-term memory to long-term memory by repeated exposure [69]. 
In the PassTiles practice mode, the password is displayed on screen, and the user can enter their password with the password either hidden or shown. They can hide or show the real password at any time during their entry attempt (for example, if they forgot one of the password tiles, they might "show" the password after entering the known tiles to help them remember the forgotten tile), and the system provides feedback about the success of each entry attempt. The expectation is that the user will create their passwords in a physically secure location where onlookers are not present. When the user feels confident that they have memorized their password, they confirm their password by completing a full, unaided entry.

An additional benefit of the practice mode is that it gives users a chance to explore how PassTiles works. They can try entering their password in multiple orders, and experiment with how the "clear password" button works. For users of Object PassTiles, the practice phase helps them understand how the shuffling works.

Another usability advantage of PassTiles is its customizable nature, that means it can be adapted to multiple use cases. For example, PassTiles seems ideal for use on a mobile device. The image size and grid dimensions can be adapted to suit the dimensions of the screen, and since no keyboard would be available on such a device, digit login would be turned off. If the system needed additional security, the system administrators might consider making the passwords ordered, to increase the security without having to make the password longer.

\subsection{Security}

To discuss the security of PassTiles passwords, let us consider a standard configuration of 48 tiles $(6 \times 8)$, with a password length of 5 randomly assigned tiles unless otherwise stated. We divide security threats into two categories: guessing attacks, where an attacker attempts to gain access to the account by making guesses at the password; and capture attacks, where an attacker attempts to learn the complete password by other means. 
Table 3.2: Theoretical password spaces for various configurations of the PassTiles password system. Note that neither the type of background image nor the login mode have any effect on the theoretical password space.

\begin{tabular}{|c|c|c|c|c|}
\hline Rows & Columns & Password Length & Ordered & Password Space \\
\hline 2 & 3 & 2 & No & $2^{4}$ \\
\hline 2 & 3 & 2 & Yes & $2^{5}$ \\
\hline 6 & 8 & 5 & No & $2^{21}$ \\
\hline 6 & 8 & 5 & Yes & $2^{28}$ \\
\hline 8 & 10 & 6 & No & $2^{28}$ \\
\hline 8 & 10 & 6 & Yes & $2^{38}$ \\
\hline
\end{tabular}

\subsubsection{Guessing Attacks}

Although the theoretical security of Image PassTiles is 21 bits in the configuration described here, various elements of these passwords are configurable. The total number of tiles may be increased by changing the grid dimensions, or passwords may be made to consist of more than five tiles. Table 3.2 shows the theoretical security of various configurations of the grid size and password length of PassTiles. Of course, as the security is increased, passwords also become correspondingly more difficult for users to remember. In configuring PassTiles, we have followed Florencio, Herley, and van Oorschot's suggestion [53] that 20 bits of entropy should be sufficient to protect against online attack.

Although we designed PassTiles to be able to easily assign passwords, it is possible to allow users to select their own password tiles. When we conducted a small study of user-chosen PassTiles passwords [127], we found that users were more likely to choose passwords with predictable patterns and hotspots. These patterns limit the effective password space of the scheme and create a vulnerability to dictionary attack.

The strength of PassTiles passwords against offline attack is dependent on how the passwords are stored. If properly salted and hashed, PassTiles passwords are not any more vulnerable to offline attack than random 21-bit text passwords.

Although digit login is meant to discourage shoulder-surfing attacks and replay attacks, it also has the effect of minimizing the password space. Because there are only 10 digits available to print on the tiles, if there are more than 10 tiles in the password grid, the theoretical password space collapses to that of an $n$-digit PIN (where $n$ is the number of tiles in the password). However, since the password changes at every 
login, an attacker must correctly guess the numeric password on the first attempt. Intersection attacks are attacks where an observer records multiple password entries from the same user, and uses the additional information to infer the password. The numeric overlap in digit login prevents intersection attacks: since multiple tile combinations may lead to the same entered PIN, an attacker cannot immediately infer the password from the entered digit string.

\section{Discretization}

One security issue for click-based graphical passwords is how to store the click points. For systems such PassPoints, Cued Click-Points (CCP), and Persuasive Cued ClickPoints (PCCP), the user chooses a particular click-point, but the system considers a login attempt successful if the user clicks within $r$ pixels of the point (forming a tolerance region around the square). However, this creates storage difficulties. Passwords should not be stored in the clear, but if the click-points are hashed, then the input click-points cannot be compared for correctness because the hashed value of nearby points will be completely different. A solution to this problem is to discretize the image into grid squares where every point inside the square is included in the tolerance region. Instead of hashing the precise click-point, the grid square is hashed, and the login click can also be hashed and compared for correctness.

There are multiple discretization schemes, including robust discretization [12] and centered discretization [31], but they all involve storing the grid offsets in the clear. While this was not initially thought to be a security vulnerability, Zhu, Wei, Yang and Yan $[149,150]$ showed that the stored grid information can be exploited to attack PCCP passwords. Using the username, initial image, and discretization grid, they created a personalized attack dictionary for each user and were able to guess $69.2 \%$ of passwords using an attack dictionary with approximately $2^{35}$ entries. They found that centered discretization better withstood the attack than robust discretization, but that both were vulnerable. They also note that the attack can be extended to CCP and PassPoints.

PassTiles avoids the discretization problems of PassPoints and PCCP. Since the grid is not secret and there are no hotspots (because of random assignment), PassTiles avoids vulnerabilities resulting from attacks on discretization schemes. Interestingly, 
the explicit grid also gives usability advantages: users can see the grid and benefit from understanding where its boundaries lie. They can choose to remember a memorable feature within each grid square (similar to PCCP's persuasive mechanism), without becoming vulnerable to hotspots. If no distinguishable feature of the square is visible, users can also choose to remember the position of the squares in relation to each other, or to the grid.

\subsubsection{Capture Attacks}

While strong enough against guessing attacks, PassTiles passwords are not as welldefended against shoulder-surfing attacks, where the attacker records the input and later enters it from the recording. PassTiles passwords are defended against casual shoulder-surfing by not displaying the user's selected tiles on the screen at login, but a more sophisticated attack with a camera could record these clicks. Because of this, we suggest that Image PassTiles is probably best suited to physically secure entry locations, rather than publicly visible places.

PassTiles passwords are the same at every login, and as such, are vulnerable to replay attacks. However, like other graphical password systems, PassTiles is moderately resistant to these accounts because the attacker must devise a methodology for recording the position of the image onscreen as well as recording the location of the clicks. This presents more difficulties than simply installing a keylogger to record text password entries. If PassTiles is configured for digit login, this further complicates replay attacks for an attacker, because the entered digits change at every login.

Phishing attacks on PassTiles are complicated slightly because the attacker needs to present the user with the correct image cue in order to get their password. However, if the phisher is able to learn the username, they may be able to fetch the user's cue in real time. Once the attacker has the cue, they can present it to the user, and record the user's clicks to reveal their password.

Like text passwords, PassTiles passwords are vulnerable to man-in-the-middle attacks unless securely transmitted using SSL. SSL encrypts the password during transmission, preventing an attacker from intercepting the transmission, reading the password (or modifying it), and passing it along to the intended end point. Of course, SSL cannot protect against compromised end points, such as in a successful phishing attack. 


\subsection{Conclusion}

PassTiles is a versatile graphical password system designed to address the usability of assigned random passwords. PassTiles takes advantage of the increased memorability of images, as well as individual image cues to increase the memorability of assigned passwords, and integrates novel features to promote the usability of these passwords. PassTiles is highly configurable, and can be customized for different security needs as well as to different practical considerations such as screen size. 


\section{Chapter 4}

\section{Memory Retrieval and Graphical Passwords}

\subsection{Introduction}

Graphical passwords are a proposed alternative to text passwords that research has shown to have good usability and security properties [11]. They can be classified into three categories, distinguished by the type of memory retrieval leveraged in the password scheme. These categories are: recall-based, cued recall-based, and recognitionbased graphical passwords. To date, there is little evidence as to which approach is best. The work in this chapter addresses this open question by investigating how different kinds of memory retrieval affect the usability and memorability of random assigned graphical passwords.

The different categories of graphical passwords [42] use different methods of information retrieval. Recall-based graphical passwords ask users to recreate a pre-set drawing to log in. Cued recall passwords show an image, and the user must click correct points on the image to log in. Recognition-based graphical passwords present users with an array of images, and the user must choose the correct images to log in.

Although psychological research has shown that recognition memory is superior to recall [62], the question of whether recognition-based graphical passwords are more memorable than other types of graphical password remains unresolved. We compare 
the three categories of graphical password in a study based in real-world usage.

Existing graphical password systems are difficult to compare, since they use different types of input and have differing levels of security. We used PassTiles (Chapter 3) to allow easy comparison of the different types of memory retrieval, and conducted a set of five studies with a total of 336 participants to investigate how leveraging different kinds of memory retrieval can affect the usability and memorability of assigned graphical passwords. We found that users were better able to remember recognitionbased passwords, but the associated login times were too slow for real-life use. In our final study, we modified the password system to allow users to take advantage of both recognition and recall memory, and these passwords were faster to enter and memorable for users. We conclude that a combination of factors need to be considered in the design of graphical passwords.

The rest of the chapter proceeds as follows: we first describe our study methodology and hypotheses. We give a summary of the results of the initial studies (more detail is available in Appendix A), and then present complete results of our followup studies. We then discuss the implications of our results, and our conclusions.

\subsection{Study Design}

The goal of our studies was to explore how recall, recognition, and cued recall information retrieval affect the memorability of assigned graphical passwords. Which of the retrieval methods is best? To investigate this question, we conducted a series of five studies. Each study was a between-subjects study, where the password scheme varied by condition and participants were randomly assigned to one condition. Participants created and used passwords on different websites over the course of one week. Although the study duration was limited, we felt it was appropriate to explore differences in this time period before more extensive testing was justified. Our studies were approved by the Carleton University Research Ethics Board.

An ongoing concern in password studies is ecological validity, or the realism of the study situation. When studying passwords, it can be difficult to know whether people exhibit the same behaviour in the study that they would in real life. In an effort to elicit realistic behaviour, we chose to study passwords in the context of website use. 


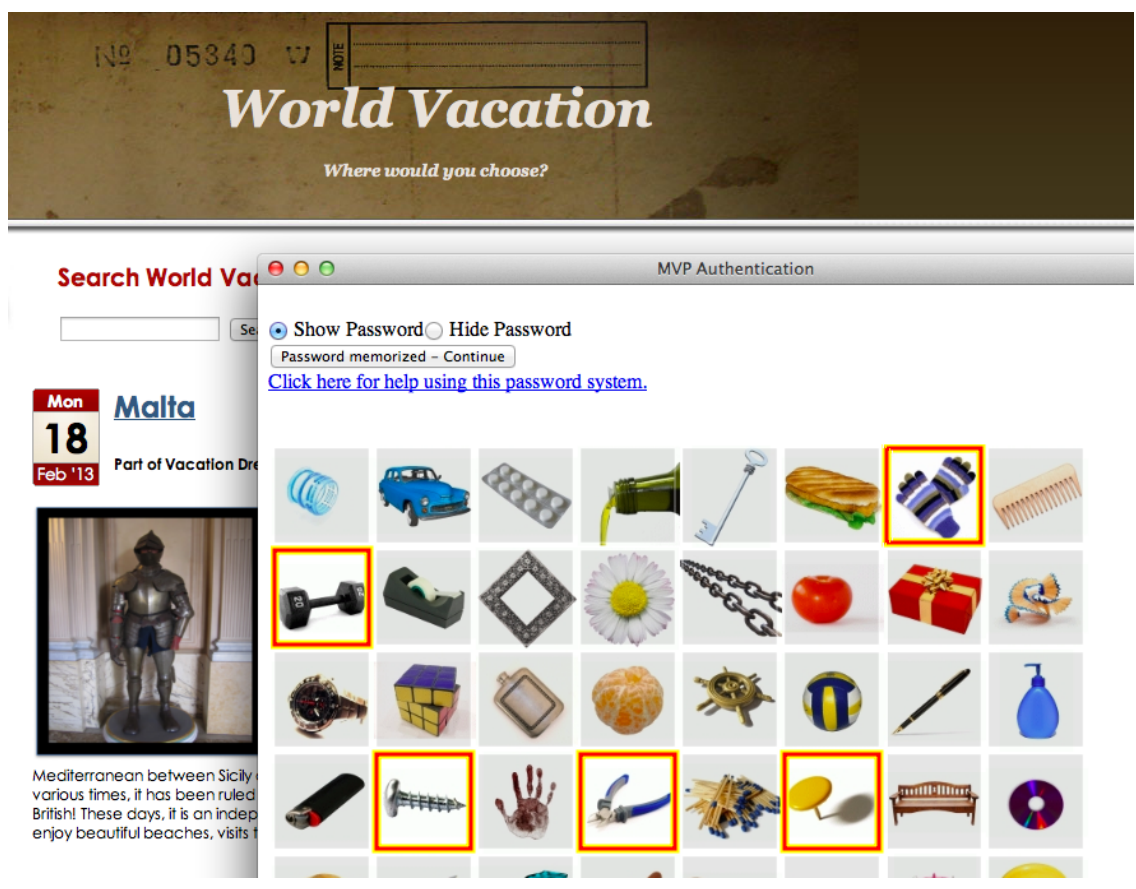

Figure 4.1: One of the three websites used in the studies, showing the PassTiles password creation screen.

In our study, we used our MVP framework [27] to implement the password systems on real websites and collect detailed usage data. Of course, novel password systems may signal the participant that the passwords are being studied, but this is unavoidable. We hope that by situating password tasks in realistic scenarios and usage contexts, the data collected can be more representative of real life use.

MVP allows different password systems to be implemented on the same websites and thus compared under identical conditions. To address privacy concerns, we created, hosted, and maintained the websites used in the study, and configured them to have dramatically different appearances. Figure 4.1 shows an example of one of these websites. We framed our study around the usability of the websites and did not put emphasis on the password systems. Participants had to log in to complete simple tasks on the websites, making the login a secondary task. We found anecdotal evidence in earlier studies [27] that users believed the scenarios: one participant asked us if she could refer her daughter to one of our websites that had advice for new university students, and another participant expressed disappointment that he had not known about one of our websites (a message board for sharing campus tips). 
Table 4.1: Password spaces for the configurations of the password systems used in the study.

\begin{tabular}{|l|c|c|}
\hline $\begin{array}{l}\text { Password } \\
\text { system }\end{array}$ & Configuration & $\begin{array}{c}\text { Password } \\
\text { Space }\end{array}$ \\
\hline BPT & $8 \times 6$ grid, length 5 & $\log _{2}\left(\begin{array}{c}6 \times 8 \\
65\end{array}\right)=21 \mathrm{bits}$ \\
IPT & $8 \times 6$ grid, length 5 & $\log _{2}\left(\begin{array}{c}\times 8 \\
5\end{array}\right)=21 \mathrm{bits}$ \\
OPT & $8 \times 6$ grid, length 5 & $\log _{2}\left(\begin{array}{c}6 \times 8 \\
5\end{array}\right)=21 \mathrm{bits}$ \\
AST & 36 characters, length 4 & $\log _{2} 36^{4}=21 \mathrm{bits}$ \\
CHT & 36 characters, length 4 & $\log _{2} 36^{4}=21 \mathrm{bits}$ \\
\hline
\end{tabular}

The five study conditions were: Blank PassTiles (BPT), Image PassTiles (IPT), Object PassTiles (OPT), Assigned Text (AST), and Chosen Text (CHT) (we use the abbreviations in tables and figures). Three conditions used PassTiles, and the remaining two study conditions used text passwords to provide comparison to a traditional password form. For the graphical password conditions, we used PassTiles to provide a common framework where only the type of memory retrieval varied. The assigned text condition gave a comparison to randomly assigned passwords, and the chosen text condition provided a controlled comparison to examine current practices and the level of security that users choose for themselves. For all of the password systems used in the study, account creation included two steps: password creation, where the user was assigned or, in the case of Chosen Text, selected their account password; and password confirmation, where the user confirmed their account password. Whether the password was assigned to or chosen by the user, this process is referred to as "password creation".

In order to a create a valid comparison, the parameters of the password schemes were set so that all five conditions had approximately equal theoretical security. Florencio, Herley, and van Oorschot [52] suggest that 20 bits of security is sufficient to guard against online attack, and we chose to use this as a guideline for the security settings in the studies. Table 4.1 shows the configuration and theoretical password spaces for the password schemes used in the studies. For example, in the PassTiles conditions, the theoretical password space is calculated as the number of combinations of 5 tiles that may be selected from the grid of 48 (i.e., $\log _{2}\left(\begin{array}{c}6 \times 8 \\ 5\end{array}\right)=21$ bits). In the case of Chosen Text, the passwords were user-chosen, but the table shows the theoretical password space. 
Each study took place in three sessions:

Session 1: Participants were briefly trained on the password system and were introduced to each of the three websites in the study, creating and confirming a password for each site. They completed a demographics questionnaire before completing a short task (such as commenting on an article) on each site. These tasks necessitated logging in to each website.

Session 2: Participants were sent three notification emails. Each email asked them to complete one task on each website. The emails were sent on the first day after Session 1, the third day, and the sixth day. Each email directed the participant to the study websites and asked them to complete a specific task on each website. Although the notification emails did not explicitly instruct participants to log in, participants needed to do so in order to complete the tasks.

Session 3: Session 3 took place one week after Session 1. Participants completed a final task on each website, and filled out the post-test questionnaire, which asked them about their experiences using the password system.

If a participant could not remember their password in Session 1, the experimenter reminded them of their original password. If they could not remember their password at any later point in the study, they were allowed to reset it. Resetting their password assigned them a new random password (using the appropriate scheme) for that account. If they were in the Chosen Text condition, resetting their password allowed the user to select a new text password.

All five studies followed the basic procedure outlined here. In Study 1, participants were recruited from our local community, and Sessions 1 and 3 were conducted inperson. Studies 2 to 5 were conducted online, using Amazon's Mechanical Turk as a participant pool. Study 4 used a modified procedure, where Session 2 was omitted and Session 3 was conducted after only 2 days. We initially used in-lab studies to tightly control the method and observe users, and later used online studies to gain access to larger numbers and more diverse participants. The five studies followed up on each other, but we never made direct statistical comparisons between studies.

The independent variable in our studies was the password system used, and its effect should indicate the usability of the scheme, including memorability and other relevant factors. We measured memorability using two dependent variables: the average length of time that a password was remembered, and the number of password resets. Our 
objective measure of usability was the login time dependent variable.

To evaluate memorability, we measured the memory time, or the average length of time that a participant remembered their password. For each account, memory time was measured as the greatest length of time between a password creation and a successful password login (using the same password). Although participants may have remembered their passwords for longer than the study, we chose this measure to give a conservative estimate of memorability. Memory time can also be influenced by when participants chose to return to tasks we set, but we have no reason to believe this would affect conditions differently. Our second measure of memorability was the number of password resets per account. The MVP system allows users to reset their passwords without experimenter intervention when forgotten. Finally, we measured login time as an indicator of each password system's usability. Login time was measured for successful password attempts from the time that the entry window appeared on screen until the website verified the entry attempt as successful. The number of login attempts was unlimited.

In a pilot study, we found that participants were repeatedly resetting their passwords rather than attempting to remember them. To subtly discourage this behaviour without introducing a monetary penalty, we introduced a 5 minute delay to the reset system and warned participants that it would take a few minutes for their passwords to be reset.

We had three initial hypotheses. Our first two hypotheses were about memorability (one concerning memory time, and one concerning password resets), and the third hypothesis was about usability (concerning login time).

H1(a): Memory time would be significantly shorter for Assigned Text than for the three graphical password conditions.

H1(b): There would be significant differences in memory time among the three graphical password conditions.

H1(c): Memory time would be significantly longer for Chosen Text than for any of the assigned password conditions.

H2(a): There would be significantly more password resets in Assigned Text than in any of the three graphical password conditions. 
H2(b): There would be significant differences in the number of password resets among three graphical password conditions.

H2(c): There would be significantly fewer password resets in Chosen Text than in any of the assigned password conditions.

H3: There would be significant differences in login times among the five study conditions.

For our analyses, we examined histograms of the distributions of each of our dependent variables, to estimate the normality of their distribution. We also examined the degree of kurtosis (flatness) and skewness of each distribution, and used this information together with the histograms to decide whether to use parametric or non-parametric statistical tests. If the distributions were approximately normal, we used parametric tests (ANOVA and $t$-tests) where appropriate, and otherwise applied ordinal tests (Kruskal-Wallis and Wilcoxon (Mann-Whitney) tests). For categorical data, we used chi-squared and Fisher's exact tests.

\subsection{Password Results}

In this section we present the results of five studies about which kind of memory retrieval was best for graphical passwords. The first two studies were conducted as part of my MA, and we summarize their results here to give context to the further studies (more detail is available in Appendix A). We first summarize the early studies, then we highlight the open problems that remained, and present our three further studies.

\subsubsection{Studies 1 and 2}

Studies 1 and 2 had the same research question: what is the best kind of memory retrieval for the design of graphical passwords? Study 1 was a hybrid study with 81 participants. Sessions 1 and 3 were conducted in-person, and participants completed 
the emailed logins in Session 2 at home. We chose this tightly controlled study design so that we could better understand participants' experiences with the websites and password systems. Participants were recruited from the Carleton University and Ottawa communities, and included students, administrative staff, and people not related to the university.

The results of Study 1 were inconclusive for memorability. We found no significant differences in memory time between the assigned text condition and each of the graphical password conditions, and we also found no significant differences among the three graphical password conditions. We also found that the chosen text passwords were not significantly more memorable than most of assigned password conditions. In all cases, it appeared that the lack of differences stemmed from a ceiling effect: almost all participants were able to remember their passwords through the entire study, leading to the lack of differences in memorability. The results for password resets were similar. The majority of participants did not reset their passwords, and we found similarly few significant differences in the hypothesis testing.

When we looked at login time as a measure of usability, we did see significant differences. Almost all pairwise comparisons were significant, and in general, text passwords (both Assigned Text and Chosen Text) were significantly faster for participants to enter and Object PassTiles passwords were significantly slower for users to enter. The Object PassTiles passwords were very slow to enter, with median login times on the order of 30 seconds. Although their memorability had been good, we started to see that their usability might be problematic.

The lack of differences in memorability was surprising to us: we had expected to see differences between conditions. In the post-test questionnaire, we asked users whether they had written down their passwords. We found that many participants had written their passwords down, particularly in the pure recall conditions (Assigned Text and Blank PassTiles), and we thought that this had probably falsely increased the memorability of those passwords.

Most of the significant differences that we did see in Study 1 were comparisons with Chosen Text. We had slight evidence that Chosen Text passwords were more memorable, and that they also had better usability. However, unlike the rest of the study passwords, the Chosen Text passwords did not have a controlled level of security. We analyzed the security of the Chosen Text passwords in our study by comparing them 
with attack dictionaries from a common password cracking tool, and found that $59 \%$ of the created passwords could be guessed with a dictionary of only 13.5 bits. This is far less than the dictionary size of 21 bits that would be required to guess the assigned passwords. Because of the difference in security levels, we feel that the memorability and usability of Chosen Text cannot be fairly compared to the assigned password conditions. In the remainder of the chapter, we report statistics for Chosen Text, but do not consider any comparison of memorability with the other conditions to be fair.

Study 2 was an online replication of Study 1 on Crowdflower [38], an outsourcing service for Amazon's Mechanical Turk [4]. Mechanical Turk is on online micro-work platform that gives access to participants from around the world. Our goal for Study 2 was to replicate Study 1 with a larger and more diverse sample.

Study 2 was conducted entirely online, and we made a few small changes to the study procedure to accommodate this fact. We created simple optional tutorial pages to help participants learn how to use PassTiles. These pages were accessed via a "Help" button on the main page.

The results of Study 2 were generally very similar to those of Study 1. Memory times were high across all conditions, and we found few significant differences in memorability. There were more password resets in Study 2 than Study 1, and we speculate that this had to do with the lack of in-person training. Once again, we found few significant differences in password resets. Login times in Study 2 were somewhat longer, but distributed similarly to Study 1. Again, there were significant differences, and we found that text passwords were significantly faster to enter than other passwords, and that Object PassTiles passwords were significantly slower to enter.

One difference between Study 1 and Study 2 was that we did not instruct participants in Study 2 not to write their passwords down. We were concerned that if we told participants not to record their passwords, they would falsely report their actual recording rates on the post-test survey. When we examined the pattern of password recording in Study 2 (Figure 4.5b), we found exactly the same pattern of frequencies as in Study 1. Although this was post-hoc analysis, the likelihood of this occurring by chance is less than 0.01, and in our subsequent studies, we formally hypothesized that we would see this same pattern of reported password recording. 


\subsubsection{Follow-up Studies}

Although previous literature assumed that using recognition would lead to the most memorable graphical passwords [42], our first studies both somewhat supported and somewhat contradicted this assumption. The results of our tests of memorability were inconclusive, and we were unable to find differences in memorability among the different graphical password conditions, even with a more representative study sample. The results of our study were clearer about usability: the recognition graphical passwords had prohibitively slow login times. Thus, the results of our studies were inconclusive, and further investigation was needed to understand the role of memory retrieval in the usability and memorability of graphical passwords.

In the subsequent studies, which are intended as contributions to the $\mathrm{PhD}$, we explored questions about whether the study design was affecting the results, and modified the design of the study to discourage confounding participant behaviour. After gaining some better understanding about how the type of memory retrieval affects the memorability of randomly assigned graphical passwords, we modified the design of the PassTiles system to better support both memorability and usability.

\subsubsection{Study 3}

The goal of Study 3 was to replicate Study 2 with more secure passwords. One possible reason for the high memorability seen in the earlier studies was that participants were simply able to remember 21 bits of randomness. To test our hypothesis, we conducted a third study where the theoretical password space of the password systems was increased to 28 bits, giving participants more information to remember (as well as more secure passwords). Study 3 followed the same methodology as Study 2, and participants were recruited from Mechanical Turk and completed the entire study online. In all studies, participants from earlier studies were excluded from participation.

For Study 3, the PassTiles passwords were reconfigured to have an $8 \times 10$ tile grid, with passwords consisting of 6 tiles. The Assigned Text passwords consisted of 6 lowercase letters, and the Chosen Text passwords were restricted to exactly 6 characters. 
There were 92 participants (35 female) in Study 3, with a median age of 27. Of these, 28 participants were students, and the majority of participants came from either India or the USA.

\section{Hypothesis 1: Memory Time}

Table 4.2: Memory time statistics (hours) (Study 3).

\begin{tabular}{|l|r|r|r|r|r|}
\hline & Mean & SD & Median & Skewness & Kurtosis \\
\hline BPT & 169.29 & 53.55 & 171.85 & -2.52 & 8.94 \\
IPT & 153.14 & 43.95 & 161.75 & -0.60 & 1.16 \\
OPT & 152.12 & 53.72 & 168.70 & -1.35 & 3.72 \\
AST & 163.99 & 33.45 & 170.95 & -1.68 & 3.67 \\
CHT & 175.46 & 15.69 & 170.78 & -0.02 & 0.24 \\
\hline
\end{tabular}

Memory times for Study 3 were very similar to those in the earlier studies, and similar across the study conditions (Figure 4.2). Although we expected that the increased password strength would lead to participants being able to remember their passwords for less time, it appeared that most participants were still able to remember their passwords for the duration of the study (Table 4.2).

Once again, we found no significant differences in memory time between Assigned Text and any of the graphical password conditions (Table 4.3), or among the three graphical password conditions $((F(2,54)=0.59, p=0.558))$.

Table 4.3: $t$-tests of memory time (Study 3).

\begin{tabular}{|l|r|r|r|}
\hline & $t$ & $\mathrm{df}$ & $p$ \\
\hline AST vs. BPT & -0.32 & 22 & 0.377 \\
AST vs. IPT & 0.87 & 35 & 0.805 \\
AST vs. OPT & 0.79 & 31 & 0.782 \\
\hline
\end{tabular}

\section{Hypothesis 2: Password Resets}

The distribution of password resets was similar to previous studies (Figure 4.3), and the majority of participants in all conditions did not reset their passwords. Surprisingly, having more secure passwords did not appear to cause participants to reset their passwords more often than in earlier studies (Table 4.4). As in Studies 1 and 2, we found no significant differences in the number of password resets between Assigned 


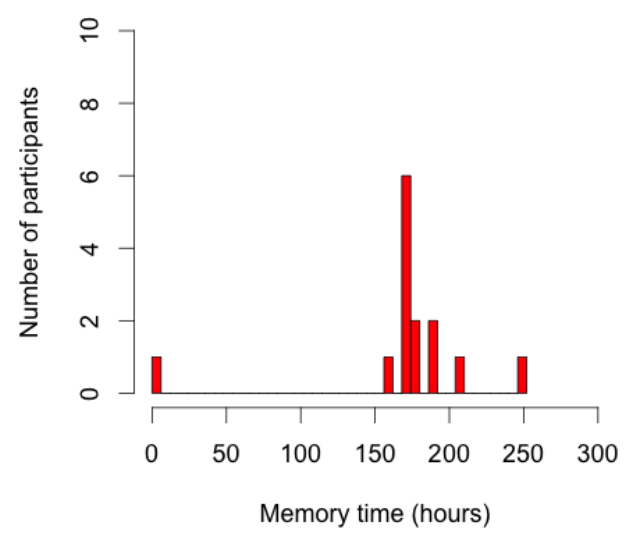

(a) BPT

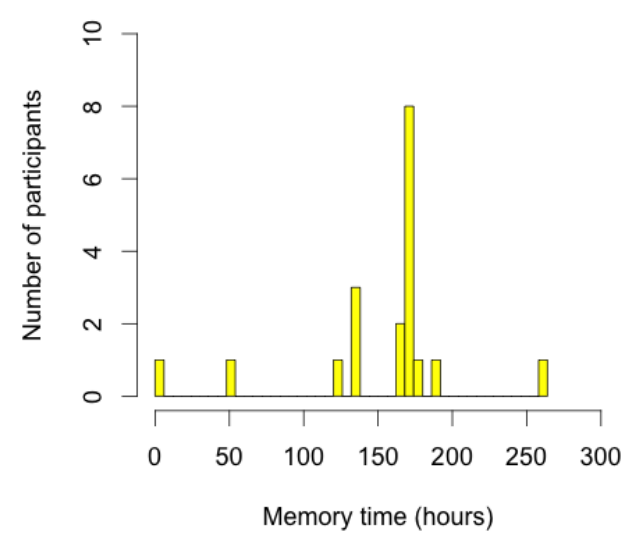

(c) OPT

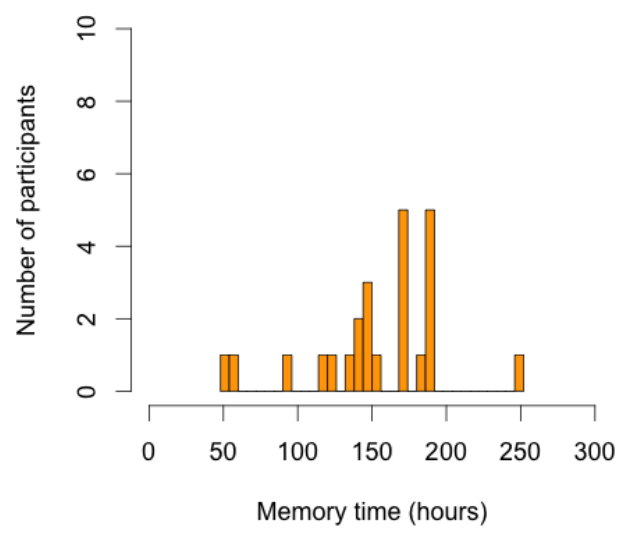

(b) IPT

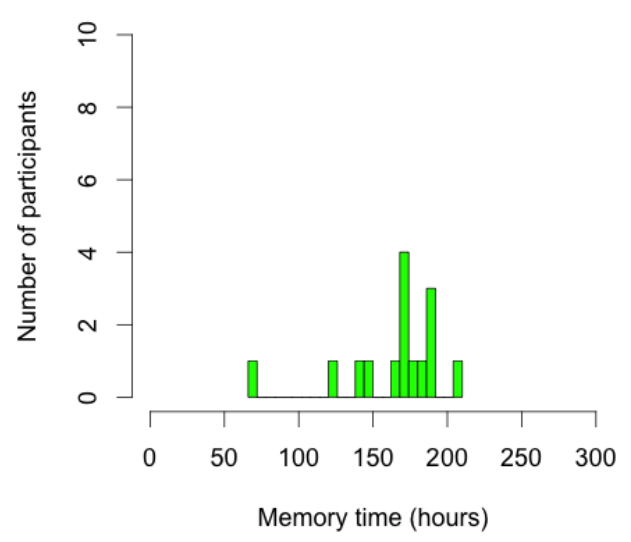

(d) AST

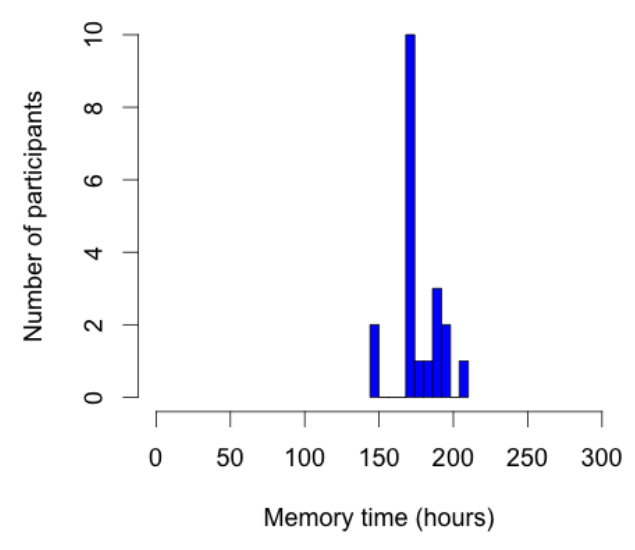

(e) $\mathrm{CHT}$

Figure 4.2: Distributions of memory time (in hours) by number of participants for each study condition (Study 3). 


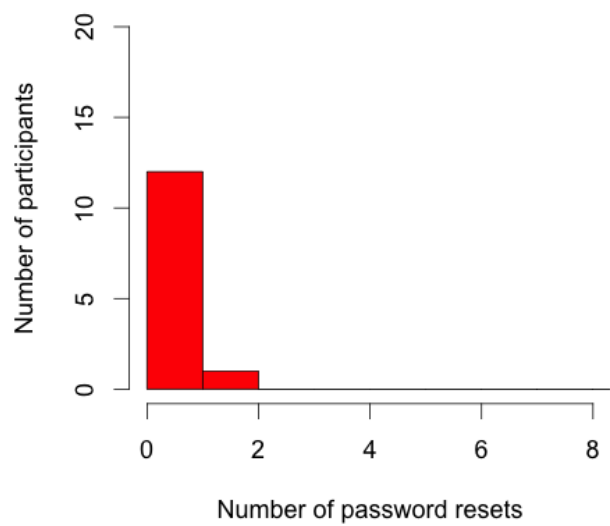

(a) BPT

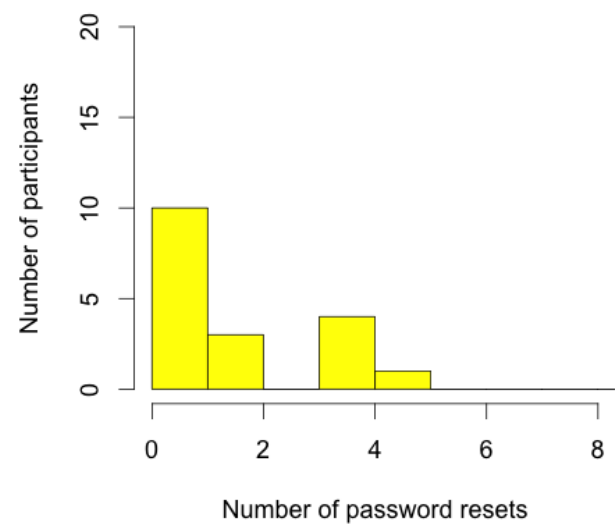

(c) OPT

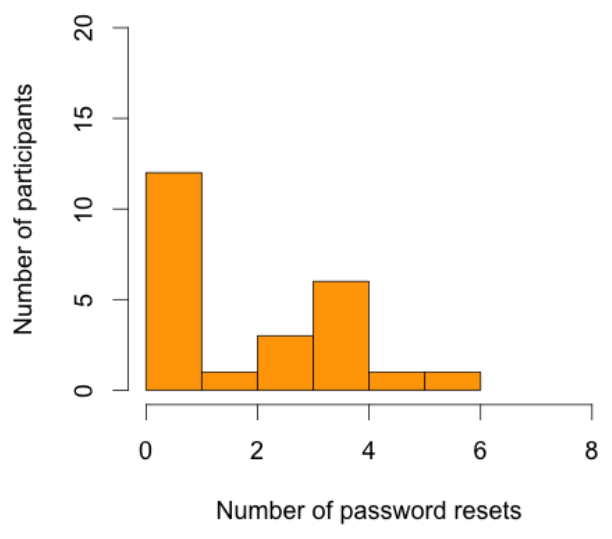

(b) IPT

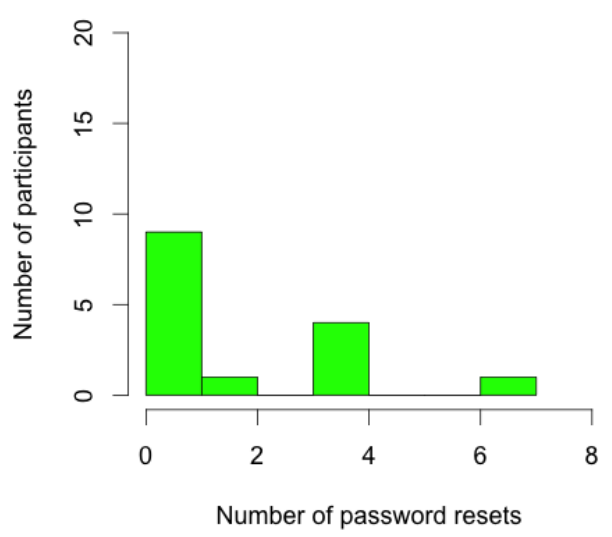

(d) AST

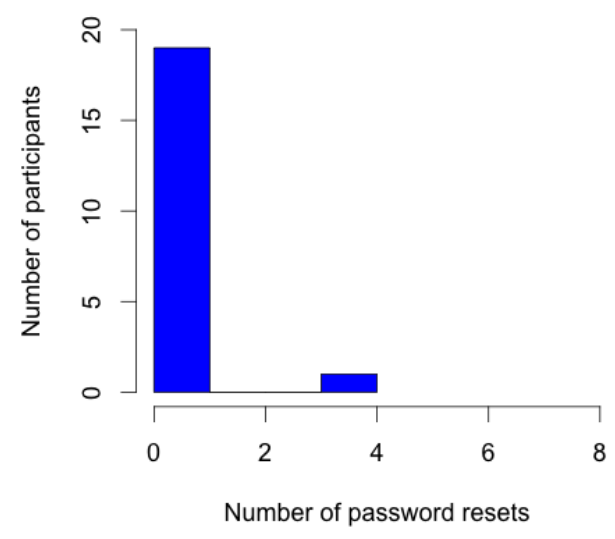

(e) $\mathrm{CHT}$

Figure 4.3: Distributions of password resets by number of participants for each study condition (Study 3). 
Table 4.4: Password reset statistics (Study 3).

\begin{tabular}{|l|r|r|r|r|r|}
\hline & Mean & SD & Median & Skewness & Kurtosis \\
\hline BPT & 0.93 & 3.20 & 0 & 3.70 & 13.76 \\
IPT & 1.42 & 1.61 & 0 & 0.60 & -0.97 \\
OPT & 1.63 & 2.87 & 0 & 2.87 & 9.75 \\
AST & 1.27 & 1.87 & 0 & 1.37 & 1.28 \\
CHT & 0.15 & 0.67 & 0 & 4.47 & 20.00 \\
\hline
\end{tabular}

Table 4.5: Wilcoxon tests of password resets comparing the assigned text condition with each of the graphical password conditions (Study 3).

\begin{tabular}{|l|r|r|}
\hline & $U$ & $p$ \\
\hline AST vs. BPT & 131.50 & 0.074 \\
AST vs. IPT & 166.50 & 0.672 \\
AST vs. OPT & 134.50 & 0.628 \\
\hline
\end{tabular}

Text and each of the graphical password conditions (Table 4.5), nor were the differences between the graphical password conditions significant $\left(\chi^{2}(2)=4.67, p=0.097\right)$.

\section{Hypothesis 3: Login times}

Table 4.6: Login time statistics (seconds) (Study 3).

\begin{tabular}{|l|r|r|r|r|r|}
\hline & Mean & SD & Median & Skewness & Kurtosis \\
\hline BPT & 33.48 & 14.44 & 33.64 & 0.11 & -0.45 \\
IPT & 33.00 & 10.21 & 32.40 & 0.65 & 0.80 \\
OPT & 58.36 & 20.92 & 50.92 & 0.97 & 0.91 \\
AST & 11.48 & 9.58 & 6.42 & 1.36 & 0.76 \\
CHT & 7.05 & 3.90 & 6.25 & 3.26 & 12.51 \\
\hline
\end{tabular}

Login times for the graphical password conditions were higher in Study 3 than in Studies 1 and 2 (Table 4.6), though similarly distributed (Figure 4.4). This increase is presumably due to having to locate and click on 6 tiles (rather than 5) and the enlarged grid size, which might affect the time taken to locate tiles. Interestingly, login times for the text password conditions remained comparable to the times seen in Studies 1 and 2 (although the passwords were longer). A Kruskal-Wallis test showed significant differences in login time $\left(\chi^{2}(3)=42.78, p<0.001\right)$, and post-hoc pairwise Wilcoxon tests (Table 4.7) showed that almost all differences were significant. 


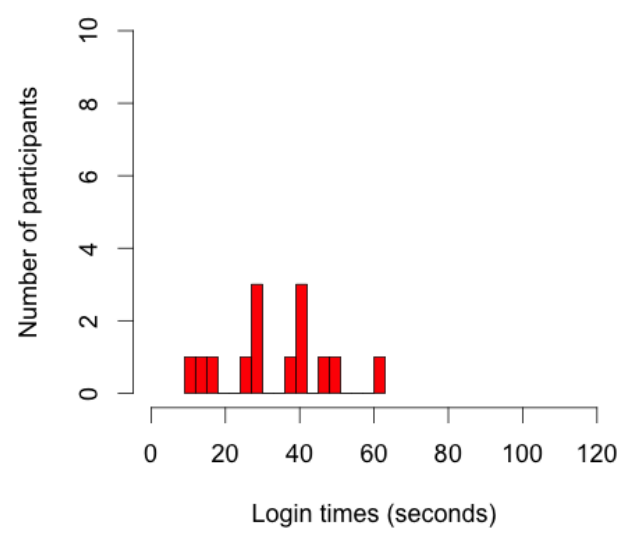

(a) $\mathrm{BPT}$

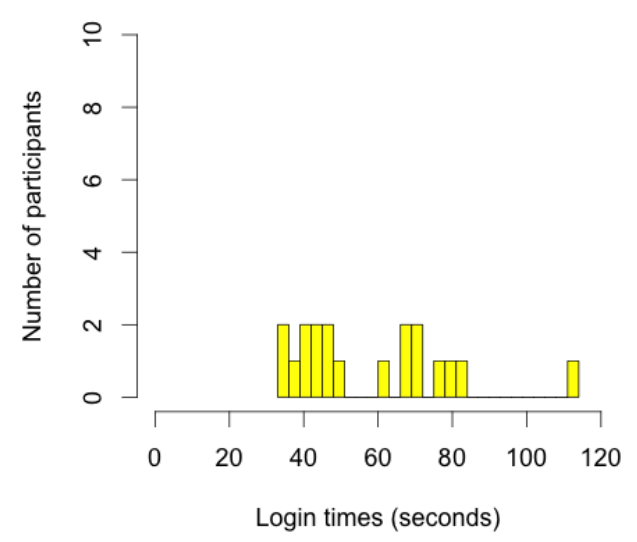

(c) OPT

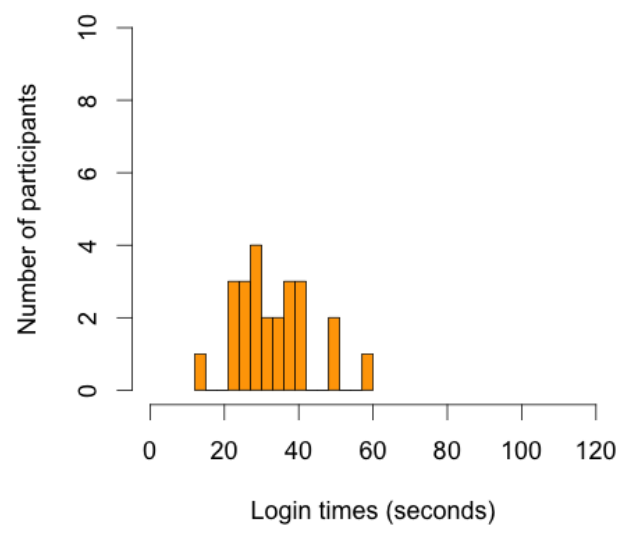

(b) IPT

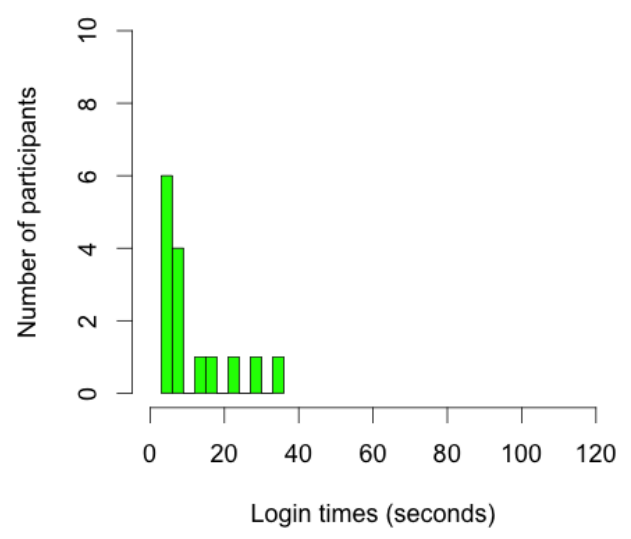

(d) AST

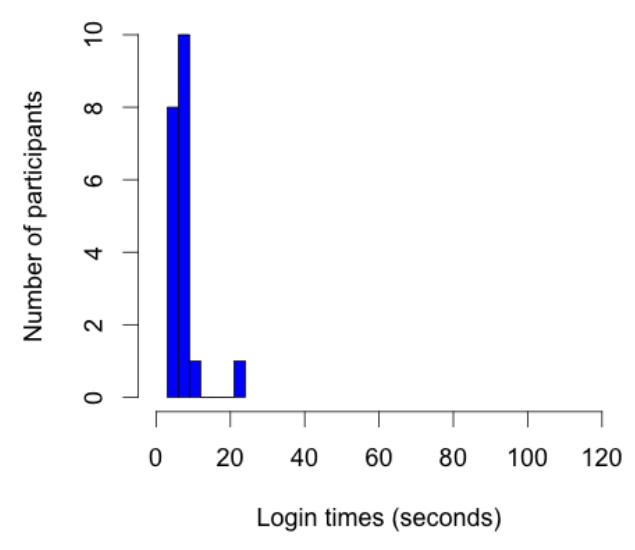

(e) $\mathrm{CHT}$

Figure 4.4: Distributions of login times by number of participants for each study condition (Study 3). 
Table 4.7: Pairwise post-hoc Wilcoxon tests of login times for assigned password conditions using Bonferroni adjustment.

\begin{tabular}{|l|r|r|}
\hline & $\mathrm{U}$ & $p$ \\
\hline BPT vs. IPT & 179.00 & 4.523 \\
BPT vs. OPT & 42.00 & 0.003 \\
BPT vs. AST & 189.50 & 0.001 \\
IPT vs. OPT & 47.00 & 0.000 \\
IPT vs. AST & 335.00 & 0.000 \\
OPT vs. AST & 284.00 & 0.000 \\
\hline
\end{tabular}

The only insignificant differences were between Blank PassTiles and Image PassTiles, and between Assigned Text and Chosen Text. The lack of difference between Blank PassTiles and Image PassTiles appears consistent across the three studies, and is likely due to the very similar physical nature of the task.

\section{Password Recording}

Figure $4.5 \mathrm{c}$ shows the reported frequency of password recording by condition. Although the numbers varied, we saw the same pattern of highest to lowest frequency that was seen in Studies 1 and 2, supporting our added hypothesis. A very high proportion of participants in Assigned Text reported writing their passwords down, and this probably accounts for the unexpectedly high memorability and low login times seen in the results.

\subsubsection{Study 4}

The goal of Study 4 was to test our hypotheses while reducing the likelihood of password recording. After conducting Studies 1 to 3, it appeared that differences in memorability were being obscured by participants writing their passwords down, and using the recorded passwords to aid their memories. While this is a legitimate technique for coping with difficult memory tasks, it makes it difficult to gain a deeper understanding of how memory for passwords works. In addition, writing passwords down can be a security risk, and from a design perspective, we wanted participants to be able to remember their passwords without writing them down. 


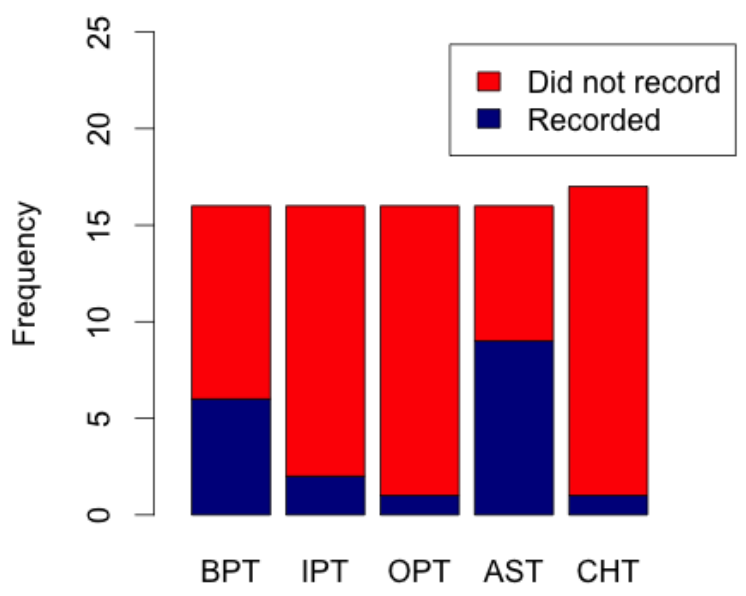

(a) Study 1

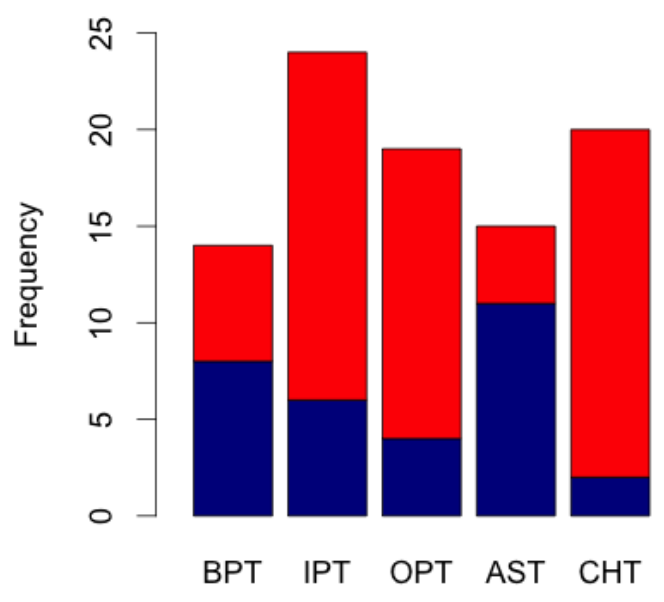

(c) Study 3

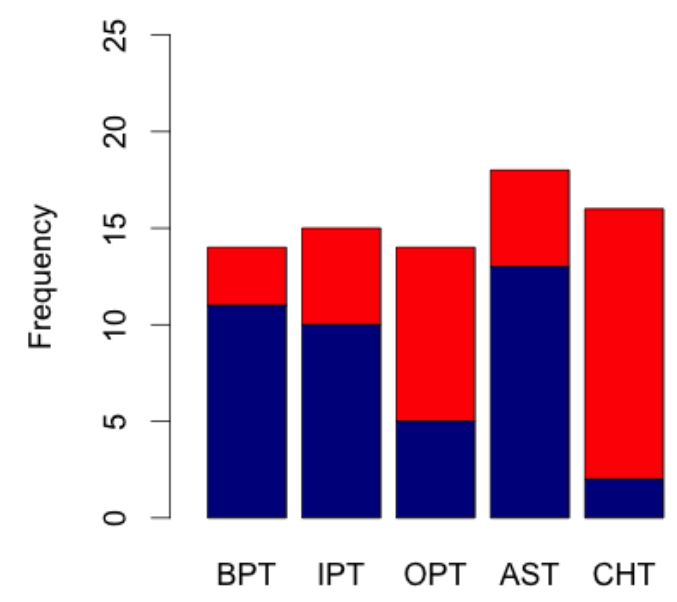

(b) Study 2

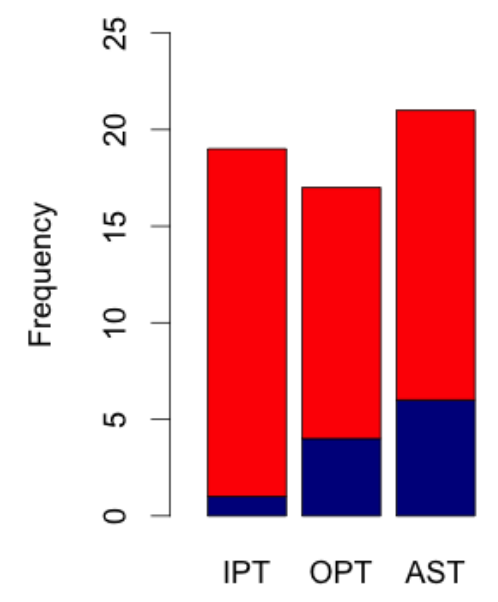

(d) Study 4

Figure 4.5: Stacked bar plots showing frequency of reported password recording. 
Table 4.8: Memory time statistics (hours) (Study 4).

\begin{tabular}{|l|r|r|r|r|r|}
\hline & Mean & SD & Median & Skewness & Kurtosis \\
\hline IPT & 13.22 & 15.85 & 16.10 & 1.27 & 1.65 \\
OPT & 22.14 & 26.42 & 0.24 & 0.59 & -1.38 \\
AST & 6.68 & 16.69 & 0.04 & 2.22 & 3.27 \\
\hline
\end{tabular}

Table 4.9: Password reset statistics (Study 4).

\begin{tabular}{|l|r|r|r|r|r|}
\hline & Mean & SD & Median & Skewness & Kurtosis \\
\hline IPT & 0.89 & 1.05 & 0 & 0.55 & -1.33 \\
OPT & 0.65 & 1.22 & 0 & 1.94 & 2.99 \\
AST & 0.90 & 1.37 & 0 & 0.95 & -1.15 \\
\hline
\end{tabular}

Study 4 was designed to investigate the same questions about how retrieval types affect password memorability, but we made a few modifications to the procedure that we hoped would discourage participants from writing their passwords down. We removed Session 2 (where participants received email requests to complete tasks on the websites), and when participants completed Session 1, we did not immediately tell them that they would be asked to complete Session 3, and were vague about the possibility of future tasks. We reasoned that if participants did not think they would need to know their passwords in future, they would not be as likely to write them down. Since intent and motivation also affect memorability [116], we decreased the total duration of the study to two days to make the memory task easier.

Study 4 used the original 21 bit passwords, and was completed entirely online using participants from Mechanical Turk. Apart from the description of the study duration, the instructions were exactly the same as those in Studies 2 and 3. Since part of our goal was to improve the design of a feasible graphical password scheme, we decided to only include the schemes showing the most promising usability and security: Image PassTiles, Object PassTiles and assigned text. There were 57 participants (23 female) in Study 4.

\section{Password Recording}

Figure 4.5d shows the frequency of reported password recording in Study 4. The modifications to the procedure appear to have had the desired effect, since a lower proportion of participants reported writing their passwords down than in Studies 1, 2, or 3. A chi-squared test of differences in password recording between conditions 


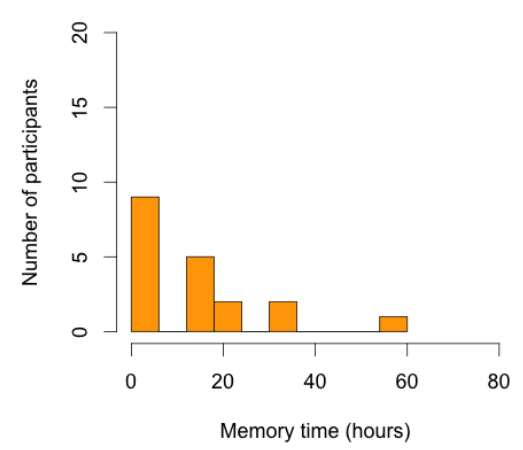

(a) IPT

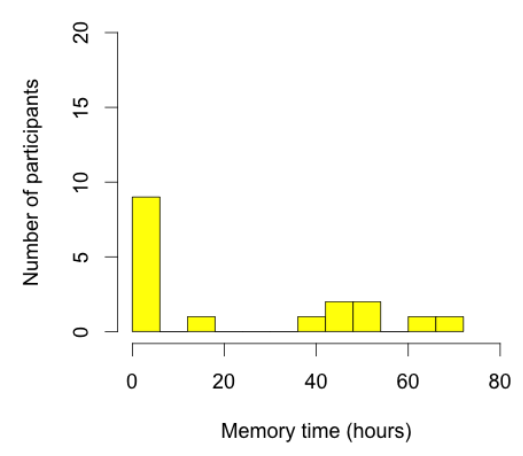

(b) OPT

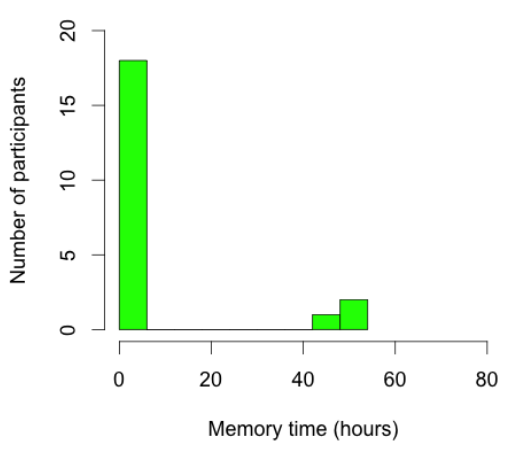

(c) AST

Figure 4.6: Distributions of memory time (in hours) by number of participants for each study condition (Study 4).

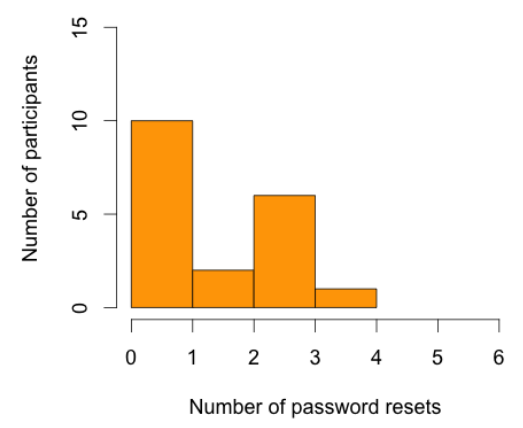

(a) IPT

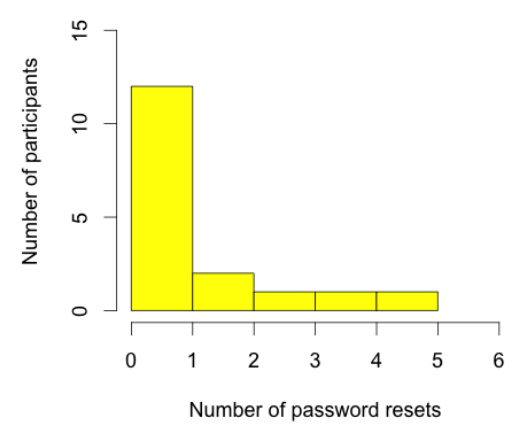

(b) $\mathrm{OPT}$

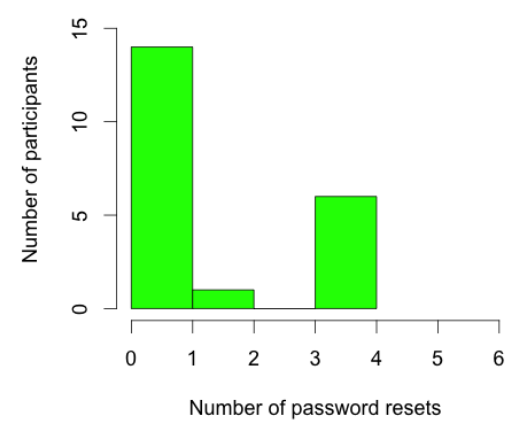

(c) AST

Figure 4.7: Distributions of password resets by number of participants for each study condition (Study 4).

in Study 4 showed no significant differences in password recording between the three study conditions. The distribution of write downs was also different than in the previous studies.

\section{Memorability}

Figures 4.6 and 4.7 show histograms of memory time and resets for Study 4. As in the earlier studies, we examined memory time (Table 4.8) and resets (Table 4.9) as measures of the memorability of the passwords. We hypothesized that there would be significant differences in memorability between the Image PassTiles, Object PassTiles and Assigned Text conditions for both memory time and number of resets. 
Table 4.10: Pairwise Wilcoxon tests of memory time using Bonferroni adjustment (Study 4).

\begin{tabular}{|l|r|r|}
\hline & $\mathrm{U}$ & $p$ \\
\hline IPT vs. OPT & 124.00 & 1.000 \\
IPT vs. AST & 270.00 & 0.574 \\
OPT vs. AST & 272.00 & 0.053 \\
\hline
\end{tabular}

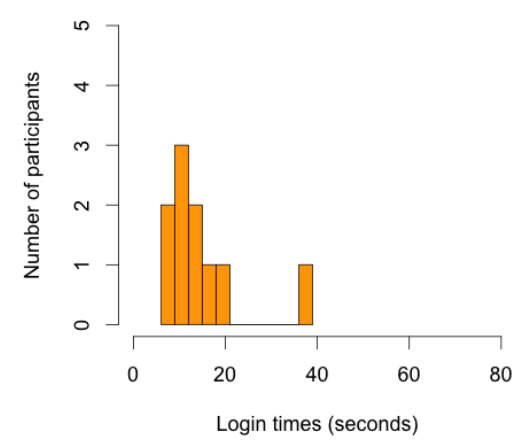

(a) IPT

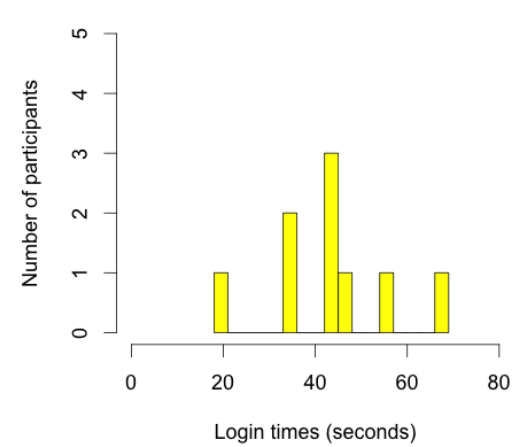

(b) OPT

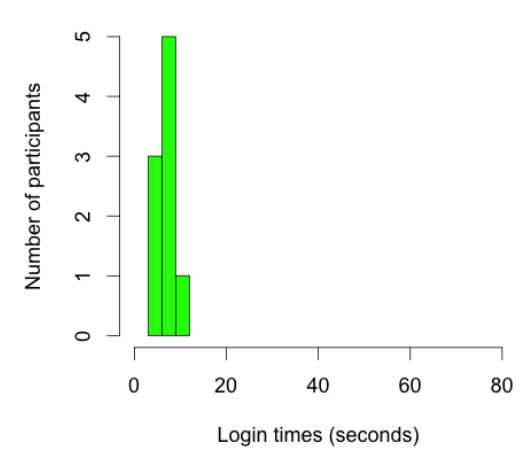

(c) AST

Figure 4.8: Distributions of login time by number of participants for each study condition (Study 4).

A Kruskal-Wallis test of memory time showed significant differences between conditions $\left(\chi^{2}(2)=8.52, p=0.014\right)$, and post-hoc Wilcoxon tests using a Bonferroni adjustment (Table 4.10) showed that participants were able to remember Object PassTiles passwords significantly longer than Assigned Text. A Kruskal-Wallis test of resets showed no significant differences between conditions $\left(\chi^{2}(2)=0.74, p=0.692\right)$.

\section{Usability}

Table 4.11: Login time statistics (seconds) (Study 4).

\begin{tabular}{|l|r|r|r|r|r|}
\hline & Mean & SD & Median & Skewness & Kurtosis \\
\hline IPT & 14.30 & 9.07 & 11.50 & 2.18 & 5.55 \\
OPT & 43.65 & 13.35 & 44.22 & 0.02 & 0.88 \\
AST & 6.59 & 1.82 & 7.00 & 0.17 & -0.53 \\
\hline
\end{tabular}

Similar to Studies 1, 2, and 3, login times were not normally distributed in Study 4 (Figure 4.8), and were longer for Object PassTiles (Table 4.11). We hypothesized that there would be significant differences in login time between the three conditions, 
Table 4.12: Pairwise Wilcoxon tests of login times using Bonferroni adjustment (Study 4).

\begin{tabular}{|l|r|r|}
\hline & $\mathrm{U}$ & $p$ \\
\hline IPT vs. OPT & 3.00 & 0.002 \\
IPT vs. AST & 81.00 & 0.037 \\
OPT vs. AST & 81.00 & 0.004 \\
\hline
\end{tabular}

and a Kruskal-Wallis test of login time showed significance $\left(\chi^{2}(2)=20.86, p<0.001\right)$. Post-hoc pairwise comparisons using a Bonferroni adjustment showed that all pairwise differences were significant (Table 4.12). These results suggest that the memorability of recognition-based passwords is superior to that of recall-based passwords.

\subsubsection{Study 5}

The goal of Study 5 was to test our hypotheses with a new condition, based on a modification of Object PassTiles. In Studies 1 to 4, it was apparent that the Object PassTiles condition had good memorability and high learnability, but its usability was severely limited by long login times. We hypothesized that the long login times were due to the shuffling feature, which put the password object images in a different position at every login, causing the user to have to spend time searching for the correct tiles. Since password tile entry order did not affect login success, we were able to gather data about the order in which participants clicked on their password tiles. We analyzed the spatial position of these tile clicks, and graphed them as heatmaps, which can be seen in Figure 4.9.

Figures 4.9a and 4.9b show the heatmaps for Blank PassTiles and Image PassTiles. In these, a clear pattern can be seen from the top left to the bottom right, indicating that participants took advantage of the spatial position of their password tiles to find and click them more efficiently. However, in Object PassTiles (Figure 4.9c), the pattern is less clear, and we speculate that this is due to participants having to search the whole screen for each object image. Since the object images are shuffled at every entry, participants were not able to rely on recall memory for the locations of their object images. Such a process would be time-consuming and would explain the long entry times seen in the Object PassTiles data. 

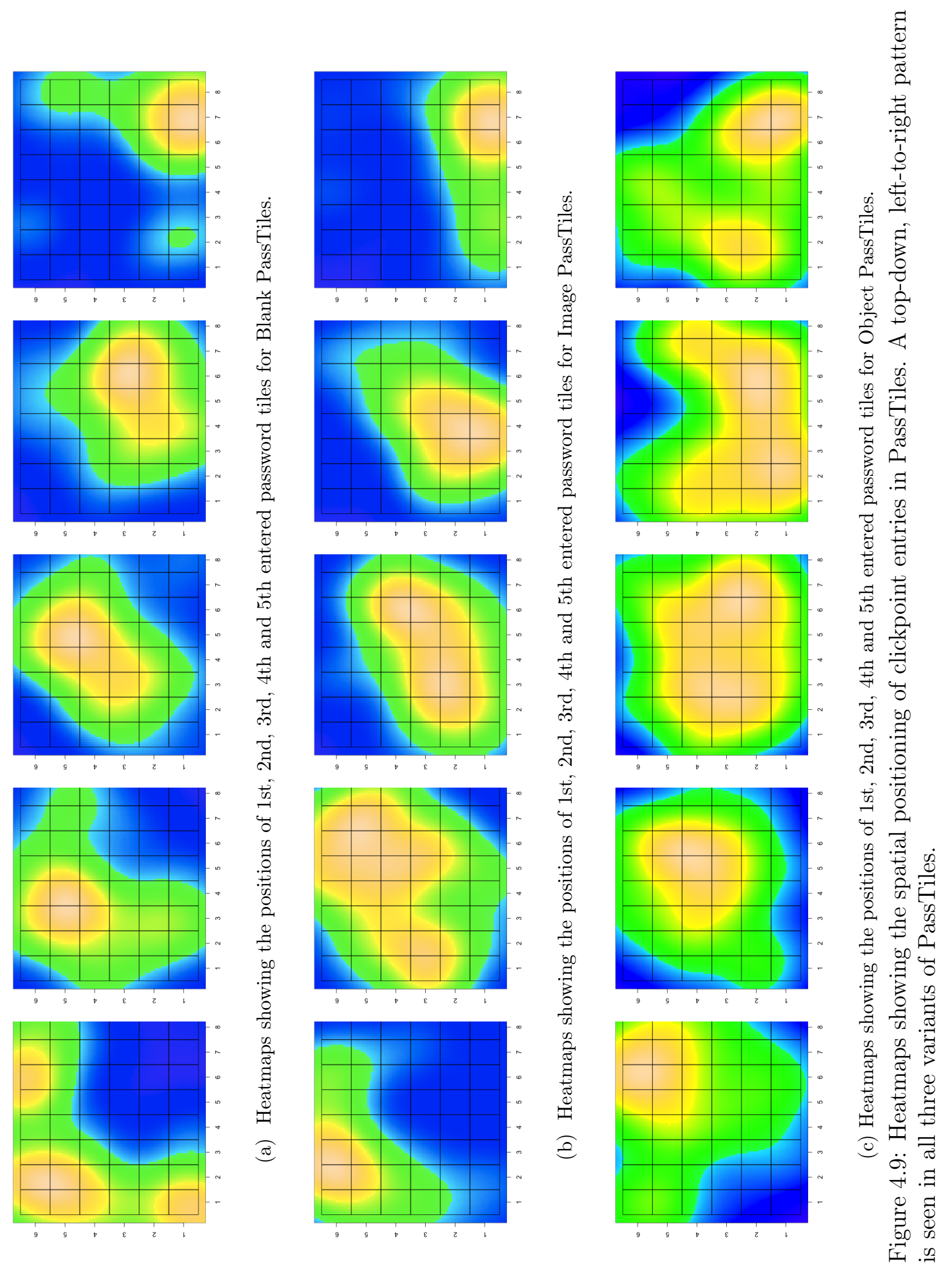
In our earlier studies, we included the shuffling feature because it forced users to rely on recognition memory, rather than recall of where the tiles were located. In deployed recognition-based graphical password systems (i.e., Passfaces), the shuffling feature has been included as a defence against shoulder-surfing attacks, but it cannot defend against capture attacks that record the password entry attempt. In an effort to decrease the login time for Object PassTiles passwords, we created a new variant of PassTiles: No-Shuffle PassTiles (NPT). No-shuffle PassTiles works exactly like Object PassTiles, but the object images remain in the same password tiles at every login, allowing users to take advantage of both recognition and recall memory when locating their password tiles.

Table 4.13: Login time statistics (seconds) (Study 5).

\begin{tabular}{|r|r|r|r|r|r|}
\hline & Mean & SD & Median & Skewness & Kurtosis \\
\hline OPT & 42.23 & 27.69 & 31.67 & 2.00 & 3.27 \\
NPT & 21.30 & 8.77 & 20.00 & 1.51 & 3.30 \\
\hline
\end{tabular}

In Study 5, we used the same basic study procedure to compare Object PassTiles with No-shuffle PassTiles. The study was conducted online, using Mechanical Turk as a source of participants. There were 29 participants (10 female). We were primarily interested in the usability of the system and the associated login times. We hypothesized that login times would be significantly longer for Object PassTiles than No-shuffle PassTiles.

Figure 4.10 shows the distribution of login times in Study 5. The distributions of login time were shown to be approximately normal (Table 4.13), and we conducted a $t$-test comparing the login times for No-shuffle PassTiles and Object PassTiles. We found that the login time for No-shuffle PassTiles was significantly shorter than for Object PassTiles $(t(19)=2.85, p=0.010)$. This result indicates that by combining recall and recognition memory, we may be able to gain good memorability with shorter login times.

\subsection{Discussion}

The goal of our studies was to explore the impact of different types of memory retrieval (recall, cued recall, and recognition) on the usability and memorability of randomly 


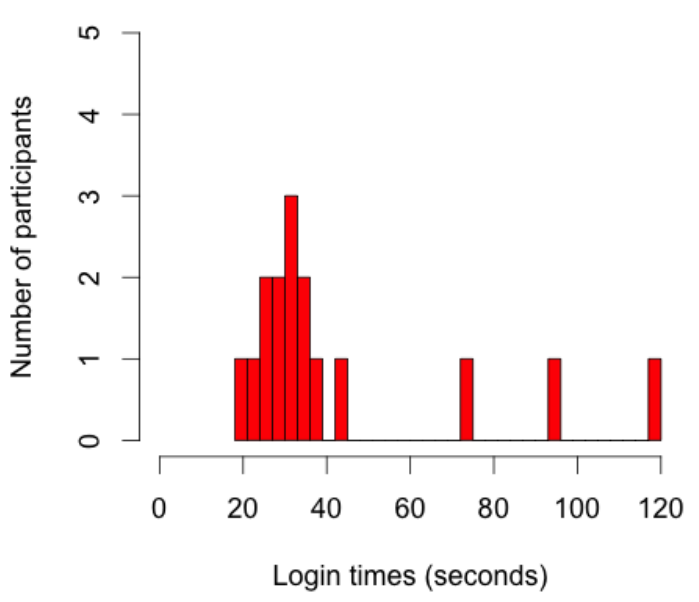

(a) OPT

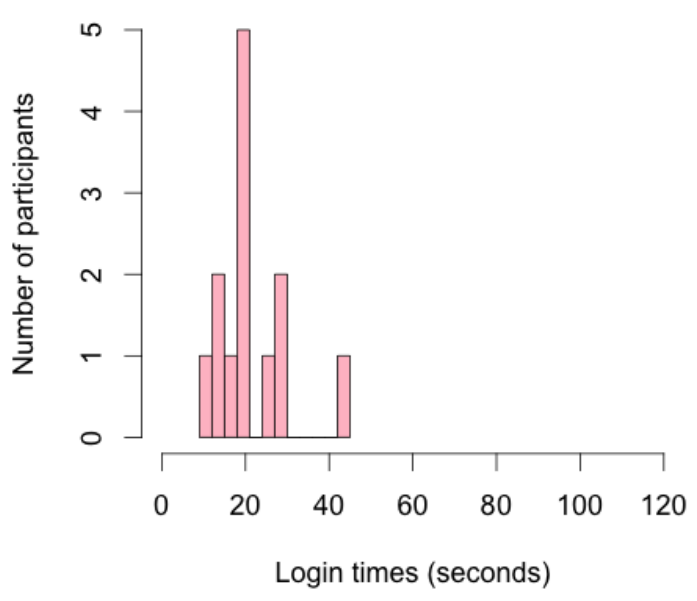

(b) NPT

Figure 4.10: Distributions of login time (in seconds) by number of participants for each study condition (Study 5).

assigned graphical passwords. Our studies showed that users were able to remember recognition-based graphical passwords better than recall-based graphical passwords. Studies 1 and 2 did not find significant differences in memorability, and when we increased the password space in Study 3, we were surprised to find that there were still few differences in memorability. It appeared that many participants were writing their passwords down, which was obscuring differences in memorability between the study conditions. When we looked at the frequency of reported password recording in Studies 1, 2 and 3, we found that fewer people reported writing their passwords down in the recognition condition (Object PassTiles), and the most people reported writing them down in the free recall conditions (Blank PassTiles and Assigned Text). We suggest that this constitutes evidence to support the hypothesis that recognitionbased graphical passwords are more memorable. In Study 4, we modified the study procedure to avoid having participants write their passwords down, and we found that participants remembered Object PassTiles passwords significantly longer than assigned text passwords. Table 4.14 shows the summarized results of our five studies, and Figure 4.5 shows the differences in password recording among the first four studies.

Although our studies showed that designing graphical password systems to use recognition memory did help users to remember their passwords, the associated login times 
Table 4.14: Summarized results of our five studies of memory retrieval. A dash (-) indicates that no differences were significant, an empty circle (o) indicates that some pairwise differences were significant, and a full circle $(\bullet)$ indicates that all pairwise differences were significant.

\begin{tabular}{|l|c|c|c|c|}
\hline \multirow{2}{*}{ Study } & \multirow{2}{*}{ Description } & \multicolumn{3}{|c|}{ Dependent Variable } \\
\cline { 3 - 5 } & & Memory Time & Password Resets & Login Time \\
\hline Study 1 & Recognition vs. recall & - & - & $\circ$ \\
\hline Study 2 & Online replication & - & - & $\circ$ \\
\hline Study 3 & Higher password strength & - & - & $\circ$ \\
\hline Study 4 & Prevent write-downs & $\circ$ & - & $\bullet$ \\
\hline Study 5 & No shuffling & & & $\bullet$ \\
\hline
\end{tabular}

were very slow. The average login time for Object PassTiles was around 30 seconds, which is too slow for widespread use. In the free recall and cued recall conditions, participants had a harder time remembering their passwords, but when they did remember them, they were able to log in faster. Although the literature on memory retrieval predicted success for recognition-based graphical passwords, it did not predict the increased login times (largely because the topic of speed is not usually addressed in the memory literature).

In Study 5, we modified Object PassTiles to remove the shuffle mechanism, and we hoped that this would allow users to leverage recognition or recall memory. We found that login times for No-Shuffle Object PassTiles were significantly shorter than those in Object PassTiles. However, the average login time was approximately 20 seconds, which was still longer than the average login time for Image PassTiles. We interpreted that participants were using a combination of recall and recognition memory when recalling their passwords.

Although different retrieval types exist on a spectrum [134], they translate to practical differences in how passwords are remembered. The higher login times in the recognition condition stem from differences in how the information is retrieved. Recognition memory involves making a binary decision for each image while traversing the entire image set. Using this decision-making process to recognize an entire password can be very slow. In contrast, recall memory involves fewer but more complex tasks. The user is less likely to successfully complete these tasks, but when successful, the process is faster. Figure 4.9 shows heatmap diagrams of where participants clicked on their $n^{t h}$ password tiles for PassTiles passwords. The heatmaps show a top-down, 
left-to-right pattern of where participants clicked on their tiles. This seems to point to the inefficiency of the search process in recognition-based graphical passwords. Since users are not able to anticipate which area of the grid to search, they resort to a time-consuming tile-by-tile approach to search for their password images.

Recognition-based graphical passwords have good memorability, but they do require some care in deployment. If password systems are designed to take advantage of recognition memory, then multiple password interference becomes a large issue. Care must be taken to ensure that image sets do not overlap, since it could be problematic if users recognized password images on the wrong website. (Our study used non-overlapping image sets.) Interference might also become an issue when resetting passwords, and it would be ideal if reset passwords had no overlapping images. However, gathering and storing large image sets adds extra work to creating and maintaining an authentication system.

Our studies showed that leveraging recognition in graphical passwords leads to good memorability, but other considerations such as login times and pragmatic concerns need to be taken into account when choosing recognition-based passwords. Different methods of memory retrieval bring different advantages and disadvantages to the memorability and usability of graphical passwords, and by designing with these affordances in mind, we can best take advantage of users' ability to remember their passwords. We conclude that cued recall memory may offer a good way of managing the conflicting demands of human memory, usability, and deployment issues. Systems such as Image PassTiles allow the use of both recall and recognition memory, while maintaining easy management of the background image sets.

\subsection{Conclusion}

Graphical passwords have been proposed as an alternative to text passwords that may have superior memorability. Three categories of graphical passwords have been distinguished on the basis of memory retrieval, but which is best has been an open question. The studies presented here investigated how the different forms of memory retrieval affect the memorability of assigned passwords. Assigned text passwords were compared to the three different kinds of assigned graphical passwords, each leveraging a different kind of retrieval: recall, cued recall, or recognition. 
The results of our studies constitute an answer to the open question of memory retrieval and graphical password design. Our studies showed that cued recall was better than free recall, and that recognition-based graphical passwords were more memorable than recall-based passwords. However, login times were slower with recognition memory. When we modified PassTiles to allow users to take advantage of both recall and recognition memory, we found that memorability was good and login times were faster. However, pragmatic issues such as password interference need to be considered, and cued recall graphical passwords such as Image PassTiles offer a good combination of memorability, usability, and manageability. 


\section{Chapter 5}

\section{The Password Life Cycle: User Behaviour in Managing Passwords}

\subsection{Introduction}

After exploring issues of memorability for assigned graphical passwords, we considered other aspects of the password management task. Passwords present several difficult tasks for users. Users are told not to create weak passwords, not to reuse passwords on multiple accounts, and not to write their passwords down. Yet users have many passwords and are expected to create a password for every new service. Often, users are required to change their passwords at regular intervals. Taken as a whole, these requirements are difficult, if not impossible, for users to meet. In response, users develop strategies for coping as best they can. We decided to try to explore and understand these strategies, in the hope of identifying new ways to alleviate the difficulties.

We conducted interviews with users to find out about their coping strategies. We asked how many accounts and passwords they have, how they create and reuse passwords, and how they handle password changes. We encouraged participants to discuss their experiences in detail, and share their motivations, fears, and password tricks.

Some findings were unsurprising. Users do write passwords down, and do reuse passwords. However, these are simplifications of their actual behaviour that do not tell 
the whole story. For example, users often write down passwords as a fallback strategy, and when they reuse passwords, they often adapt them for different accounts. We analyzed our interviews using the Grounded Theory methodology and identified some important patterns in user behaviour. We identified a "life cycle" of password use, where the user's central concern is rationing effort to best protect important accounts. Many of the specific practices are already known, and our contribution is the identification of a coherent model that highlights a consistent series of gaps between user behaviour and current tool support. We describe the emergence of this model, and suggest that it can inform better ways to support users in their behaviour, rather than providing unrealistic guidance.

In the following sections, we describe our methodology and the details of our interviews. Section 5.3 presents an overview of the results, and Section 5.4 documents the step-by-step process of our qualitative analysis. We then suggest some implications of our findings, and our conclusions.

\section{$5.2 \quad$ Study}

To investigate how users manage and keep track of their passwords, we conducted a series of interviews about password habits. We conducted the interviews ourselves, which included asking the questions, recording answers, and encouraging participants to discuss or give fuller answers. The interviews were audio-recorded to allow further note-taking and analysis. We also conducted a brief self-administered demographics questionnaire that collected basic information including age, gender and occupation, and was mostly intended to give a better understanding of the interview sample. Our questionnaires can be found in Appendix B, and this study was approved by our ethics board.

We developed our interview questionnaire around the idea of exploring users' password management techniques. We asked a set of general questions about password habits and usage, including questions about how many passwords and accounts participants had, whether they reused passwords, whether they used password managers, and how they kept track of their passwords. The next series of questions asked about how they would behave when creating new accounts, and when changing or resetting 


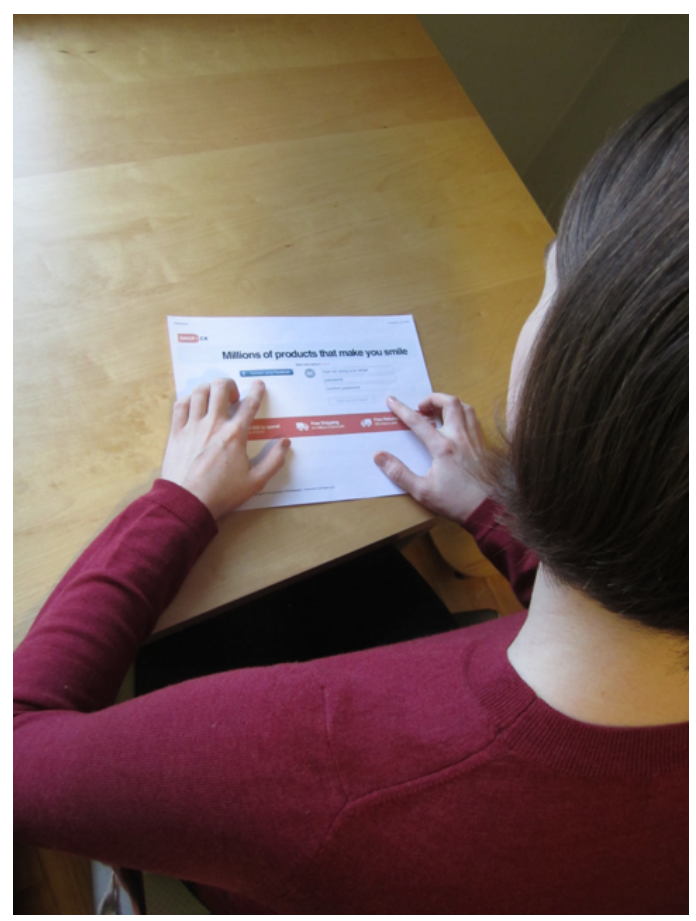

Figure 5.1: Participants were provided with cards showing website screenshots. The purpose of these cards was to let participants better immerse themselves in the interview questions by imagining that they were creating an account on the pictured websites.

the password on an existing account. We did not ask participants what their passwords were, and we specifically told participants that they should never reveal their passwords to us. We used the constant comparative approach [128] when conducting the interviews, where we refined the focus of the interview discussion throughout the study. Each interview took approximately 30 minutes, and the interviews were conducted at our university.

We chose our methodology to encourage participants to discuss thoughtfully the ways in which they approach the task of password management. We used a guided interview to focus the discussion around topics of interest to us, but we asked additional questions to probe responses and follow up on emerging topics of interest. We broke questions into a number of parts to give participants an opportunity to fully explain how and why they make their decisions, and to avoid having participants rush through their answers. We provided users with props in the form of cards with website screenshots (Figure 5.1) to situate themselves in the password creation and reset tasks, and to encourage them to consider their real life behaviour. 
We used Grounded Theory [128] to analyze the interview data and conduct qualitative analysis of participants' responses and discussion. Grounded Theory is an analytical framework that seeks to develop an explanatory theory from a set of data. It builds a theory grounded in evidence, rather than validating an outside theory or testing generalizability. Grounded Theory defines a theory as:

"... a set of well-developed categories (e.g., themes, concepts) that are systematically interrelated through statements of relationship to form a theoretical framework that explains some relevant social, psychological, educational, nursing, or other phenomenon." (p.22 [128])

\subsection{Results Overview}

We interviewed 27 participants for the study, and all were recruited from the university community via posters, mailing lists, and word-of-mouth. At 27 participants, we reached saturation, where we were hearing little new from additional participants.

Two-thirds of participants were female. Participants' age ranged between 17 and 67, with a median age of 22. Most participants were either full- or part-time students, and came from a range of programs including the humanities, sciences, and social sciences. None of the participants were studying computer science or computer security. The other participants worked in the university community, in roles such as administrative assistants, librarians, and security guards.

In addition to the deeper responses and discussion, the interview questions also yielded a set of quantitative data about how many passwords and accounts users have, how many passwords they reuse, and the extent to which they use password managers and other tools. We present below descriptive statistics of the responses to these questions. We present these data before the Grounded Theory analysis to give context to participants' responses.

The first part of the interview investigated how many accounts and passwords users have. We wanted participants to closely reflect on their answers to these questions, so we divided questions into multiple parts. For example, in a question about number of accounts, we identified 14 account categories where participants might have accounts, and asked about each category individually. We hoped this technique would help users remember infrequently used accounts. 
Participants reported their total number of accounts as being between 9 and 51 accounts, with a median of 27 accounts. The bulk of most participants' accounts consisted of email addresses, school or work accounts, and social networking accounts. They reported using between 3 and 14 accounts in an average week, with a median of 11 accounts. Our account category elicitation technique yielded numbers somewhat higher than most other studies [23,57,98,63], but in line with the results found in Florencio and Herley's 2007 study [50]. Although our approach did seem to help participants remember more accounts, we still suspect that participants were not able to list all of their active accounts.

Participants reported having between 2 and 20 unique passwords, with a median of 5 passwords. All but one of the participants in the study (26 participants, $96 \%$ ) reported reusing passwords between accounts. Of the participants who reported reusing passwords, most (23 participants, 88\%) reported reusing more than one password, and 19 (73\%) reported reusing passwords either "always" or "frequently". Participants described different strategies for reusing passwords. Some described using the same password for all accounts, and others described linking passwords with usernames. Participants also reported using different passwords for different online contexts, such as at work or school. Several participants mentioned that they were careful not to reuse passwords on "financial" or "important" accounts (though many did not clarify what was important). Conversely, many participants also mentioned having a specific password that they reused widely on accounts of low interest, low importance, or infrequent use.

We were interested in whether participants considered context of use in their password management strategies. Participants reported entering their passwords on a range of devices including desktop computers, laptop computers, tablets, e-readers, and smartphones, but the most commonly reported context was a computer (laptop or desktop) and a smartphone. Most participants $(18,66 \%)$ said that they did not consider device constraints when choosing passwords. Participants who considered device constraints mentioned that they checked the availability of apps (to reduce the difficulty of password entry), the different security requirements of different devices, and awareness of the usability of different keyboards. All participants reported that they enter their passwords on computers that do not belong to them. Several participants mentioned that they were more careful about logging out on these computers, and about not saving passwords in the browser. One participant mentioned that they 
sometimes changed their passwords after entering them on computers belonging to other people.

The next set of questions addressed the coping strategies that users develop to keep track of passwords and accounts. We asked participants if they used any kind of password manager (including the browser-based managers), and 22 respondents (81\%) said that they saved their passwords in some kind of password manager. All of these went on to clarify that they saved passwords in their browser or in the Apple Keychain. No one reported currently using dedicated password management software, although one participant said that they had previously used one. We also asked if participants ever clicked the "remember me" button to stay logged in to websites using cookies, and 22 participants (81\%) reported clicking these boxes. Although the same percentage of participants said they used cookies as password managers, these sets did not completely overlap.

Twenty-one participants $(78 \%)$ reported writing down at least some of their passwords. Of these participants, most referred to the recorded passwords as a backup for memory, and not a resource used at every login. Participants reported different recording strategies - some recorded only part of the password, or a hint to the password, while others were more methodical about recording all of every password. Participants reported using both physical and digital media to store passwords, but specified that the recorded passwords were easily accessible from their regular computing context.

The final part of the interview asked participants about password changes and resets of forgotten passwords. Forty percent of participants reported having ever changed passwords of their own volition, and these participants remarked that they changed passwords rarely, and only under special circumstances. Most participants evidently did not consider situations where they changed forgotten passwords, because all participants reported having done this. Most participants reported resetting forgotten passwords once per month or less, and most people said that their strategy in those cases was to change the password to something similar to existing passwords (often reusing or adapting an existing password). 


\subsection{Qualitative Analysis}

We chose not to fully transcribe our recordings. Instead, we made detailed notes about responses to the interview questions. These notes included quantitative question responses, but also included additional details from participants' discussion of the topic. In places where our notes were not sufficiently detailed, we returned to the audio-recorded data for additional information. We referred to the audio-recordings to transcribe exact quotes for use in this chapter.

For the qualitative analysis, we followed the Grounded Theory methodology of Strauss \& Corbin [128]. This method involves several steps in the analysis process. First, recorded data is analyzed point-by-point and assigned descriptive codes, in the process of open coding. Next, these codes are compiled, and the process of axial coding looks for relationships among the codes. In the process of axial coding, the researcher asks questions such as why, where, how, and when in an effort to uncover structure in the data. Finally, selective coding integrates the results of the open and axial coding, and refines them into a theory.

\subsubsection{Open Coding}

We began the process of Grounded Theory by developing a set of descriptive open codes. We generated the open codes by examining the noted responses from the interview data. We traversed the answers to each question, looking for recurring patterns and themes in the data. Each of these themes was denoted by a code. We had a total of 67 codes (Table 5.1).

Some of the codes emerged in relation to the question being asked in the interview. For example, we asked participants about whether they wrote their passwords down, and how they stored and referred to recorded passwords. Several codes about password recording emerged from responses to that question. However, other codes emerged over the sequence of the discussion. Participants gradually revealed more about their password creation, organization and categorization techniques as we explored how they would handle password creation, how they would choose passwords for new accounts, and how they would keep track of new accounts. 
One of our password recording codes was records passwords as backup strategy, and we used this code when a participant indicated that although they wrote at least some of their passwords down, they did not refer to these recorded passwords on a regular basis, and instead appeared to use the recorded passwords as a backup.

In the following quote, the participant describes how she used to write her passwords down as a fallback for memory when going on vacation.

"Not any more. I used to. [Why did you stop?] Because the only reason to write them down was if I was going on vacation for two weeks and I'd come back to work and I wouldn't remember my password [laughs]. So that was [garbled] but now I very rarely take vacation more than one week at a time and I can remember one week [laughs]."

$-\mathrm{P} 15$

This participant describes writing her work passwords down so that she would be able to remember them after a long delay. However, she does not need this technique in everyday use. She also explains how a change in her circumstances (shorter vacations), has affected her password coping strategies (coded as change of habit).

Another code was single sign-on which we used when a participant brought up the subject of single sign-on services, such as through Facebook or Google.

"Or you could just connect through Facebook. [It's true. Would you/do you do that?] I actually don't do that very often, no. [Why not?] Uhh, I just find there's so much junk on Facebook sometimes that I really just don't want to add to it. On Facebook, I try to only add, umm, things that are more important to me I guess." - P27

This participant shows a misunderstanding of single sign-on when she explains that she avoids signing in through Facebook because she thinks her activities will be posted to her Facebook feed. Interestingly, she does not express concerns about privacy, but rather about the relevance of information that she posts to her personal page. 


\subsubsection{Axial Coding}

Following open coding, we began the process of axial coding. In axial coding, we took the codes assigned in open coding and looked for patterns, connections, and relationships between those codes. Our eventual goal was to form a model or theory that described our data. To examine the codes, we tagged each code with a post-it note, and arranged them on a table to look for connections (Figure 5.2).

Table 5.1: The 67 codes used in the open coding process. The codes are organized into related groups based on the initial step of the axial coding process.

\begin{tabular}{|c|c|}
\hline Code Name & Description \\
\hline \multicolumn{2}{|l|}{ Contextual Behaviour } \\
\hline Important accounts & $\begin{array}{l}\text { Has accounts that are more important that } \\
\text { treats more carefully. }\end{array}$ \\
\hline Banking as important account & $\begin{array}{l}\text { Banking was a frequently distinguished impor- } \\
\text { tant account. }\end{array}$ \\
\hline $\begin{array}{l}\text { Contextual behaviour for dif- } \\
\text { ferent websites }\end{array}$ & $\begin{array}{l}\text { Different password behaviour on different web- } \\
\text { sites. }\end{array}$ \\
\hline $\begin{array}{l}\text { Contextual behaviour for dif- } \\
\text { ferent devices }\end{array}$ & $\begin{array}{l}\text { Different password behaviour on different de- } \\
\text { vices. }\end{array}$ \\
\hline $\begin{array}{l}\text { Contextual behaviour for dif- } \\
\text { ferent environments }\end{array}$ & $\begin{array}{l}\text { Different password behaviour in different places } \\
\text { (e.g.,, work vs. home). }\end{array}$ \\
\hline \multicolumn{2}{|l|}{ Password Categories } \\
\hline Categorizes by security & $\begin{array}{l}\text { Categorizes password reuse by the level of secu- } \\
\text { rity. }\end{array}$ \\
\hline Categorizes by password rules & $\begin{array}{l}\text { Categorizes password reuse by the password } \\
\text { rules on the website. }\end{array}$ \\
\hline $\begin{array}{l}\text { Categorizes by affective impor- } \\
\text { tance }\end{array}$ & $\begin{array}{l}\text { Categorizes passwords by the personal impor- } \\
\text { tance of the account }\end{array}$ \\
\hline Categorizes by frequency & $\begin{array}{l}\text { Categorizes password reuse by the frequency of } \\
\text { website use. }\end{array}$ \\
\hline Categorize & Categorizes accounts by content similarity. \\
\hline $\begin{array}{l}\text { Passwords linked to password } \\
\text { rules }\end{array}$ & $\begin{array}{l}\text { Creates passwords to use with different pass- } \\
\text { word policies. }\end{array}$ \\
\hline
\end{tabular}

Continued on next page 
Table 5.1 - Continued from previous page

\begin{tabular}{|c|c|}
\hline Code Name & Description \\
\hline Affective passwords & $\begin{array}{l}\text { Picks passwords that have emotional signifi- } \\
\text { cance. }\end{array}$ \\
\hline $\begin{array}{l}\text { Personal information in pass- } \\
\text { words }\end{array}$ & $\begin{array}{l}\text { Incorporates personal information into pass- } \\
\text { words. }\end{array}$ \\
\hline $\begin{array}{l}\text { Infrequently used passwords } \\
\text { are hard to remember }\end{array}$ & $\begin{array}{l}\text { Finds it difficult to remember passwords for in- } \\
\text { frequently used accounts. }\end{array}$ \\
\hline \multicolumn{2}{|l|}{ Creating Passwords } \\
\hline Algorithmic passwords & $\begin{array}{l}\text { Uses some kind of algorithm to generate pass- } \\
\text { words. }\end{array}$ \\
\hline Reused password chunks & $\begin{array}{l}\text { Reuses parts of passwords across different ac- } \\
\text { counts (perhaps in combination). }\end{array}$ \\
\hline Variations on a theme & $\begin{array}{l}\text { Unique passwords consist of variations on a sin- } \\
\text { gle password. }\end{array}$ \\
\hline Passwords linked to usernames & Associates passwords with unique usernames \\
\hline Passwords linked to email & $\begin{array}{l}\text { Associates passwords with email addresses (of- } \\
\text { ten when using email addresses as usernames). }\end{array}$ \\
\hline Passwords linked to devices & $\begin{array}{l}\text { Associates passwords with the device that they } \\
\text { access the account from (or created the account } \\
\text { on). }\end{array}$ \\
\hline Passwords linked to times & $\begin{array}{l}\text { Associates passwords to the time period in } \\
\text { which they were created (e.g.,, during under- } \\
\text { grad). }\end{array}$ \\
\hline $\begin{array}{l}\text { Passwords linked to website } \\
\text { content }\end{array}$ & $\begin{array}{l}\text { Links passwords to content found on the web- } \\
\text { site, or reason for visiting the website. }\end{array}$ \\
\hline Affective passwords & Creates passwords with emotional significance. \\
\hline $\begin{array}{l}\text { Personal information in pass- } \\
\text { words }\end{array}$ & $\begin{array}{l}\text { Creates passwords with personal information } \\
\text { (i.e., phone numbers, birthdays). }\end{array}$ \\
\hline Cycles through passwords & $\begin{array}{l}\text { When creating new accounts, uses each of their } \\
\text { passwords in turn. }\end{array}$ \\
\hline Named Passwords & $\begin{array}{l}\text { Has a specific nickname for their most fre- } \\
\text { quently used password. }\end{array}$ \\
\hline
\end{tabular}

Continued on next page 
Table 5.1 - Continued from previous page

\begin{tabular}{|c|c|}
\hline Code Name & Description \\
\hline "Familiar Password" & $\begin{array}{l}\text { Referred to one of their passwords as being most } \\
\text { "familiar". } \\
\text { When fitting password to password policies, has } \\
\text { a set of habitually used numbers and symbols } \\
\text { that they add. }\end{array}$ \\
\hline \multicolumn{2}{|l|}{ Password Recording } \\
\hline Digital Recording & $\begin{array}{l}\text { Records passwords in digital media (e.g.,, in } \\
\text { email, or in an excel file). }\end{array}$ \\
\hline Physical Recording & $\begin{array}{l}\text { Records passwords in physical media (e.g.,, on } \\
\text { post-it notes, in a journal). }\end{array}$ \\
\hline $\begin{array}{l}\text { Recording as occasional strat- } \\
\text { egy }\end{array}$ & Records passwords only occasionally. \\
\hline Records as backup strategy & $\begin{array}{l}\text { Records passwords, but does not refer to them } \\
\text { consistently. }\end{array}$ \\
\hline Records temporarily & $\begin{array}{l}\text { Records passwords as a temporary measure } \\
\text { (e.g.,, when going on vacation). }\end{array}$ \\
\hline Always records & Systematically records all of their passwords. \\
\hline Records when special policies & $\begin{array}{l}\text { Records passwords when the website policy pro- } \\
\text { hibits resets or disables cookies. }\end{array}$ \\
\hline Records assigned passwords & Records system-assigned passwords. \\
\hline $\begin{array}{l}\text { Records passwords for infre- } \\
\text { quently used accounts }\end{array}$ & $\begin{array}{l}\text { Records the passwords on accounts that does } \\
\text { not use often. }\end{array}$ \\
\hline Records for memorability & Records passwords to help remember them. \\
\hline Records clues to password & Records hints or clues about the password. \\
\hline Records usernames & $\begin{array}{l}\text { Records usernames (either with or without as- } \\
\text { sociated passwords). }\end{array}$ \\
\hline Tools & \\
\hline $\begin{array}{l}\text { Treats cookies as password } \\
\text { manager }\end{array}$ & $\begin{array}{l}\text { Uses the "remember me" (cookie) mechanism } \\
\text { interchangeably with the browser password } \\
\text { manager. }\end{array}$ \\
\hline
\end{tabular}


Table 5.1 - Continued from previous page

\begin{tabular}{|c|c|}
\hline Code Name & Description \\
\hline Treats cookies differently than & Distinguishes the "remember me" from the pass- \\
\hline password manager & word manager and uses differently. \\
\hline $\begin{array}{l}\text { Uses tools only in some con- } \\
\text { texts }\end{array}$ & $\begin{array}{l}\text { Uses cookies or browser password managers } \\
\text { only on some devices. }\end{array}$ \\
\hline $\begin{array}{l}\text { Combination of coping strate- } \\
\text { gies }\end{array}$ & $\begin{array}{l}\text { Uses a combination of coping strategies to re- } \\
\text { member passwords (e.g.,, password manager, } \\
\text { writes passwords down, and password resets). }\end{array}$ \\
\hline $\begin{array}{l}\text { Unable to take advantage of } \\
\text { coping strategies }\end{array}$ & $\begin{array}{l}\text { For some reason, cannot use a certain tool or } \\
\text { technique to remember their passwords. }\end{array}$ \\
\hline Strength meter & $\begin{array}{l}\text { Evaluates password strength on a strength me- } \\
\text { ter if present at password creation. }\end{array}$ \\
\hline $\begin{array}{l}\text { Rehearses passwords by log- } \\
\text { ging in }\end{array}$ & $\begin{array}{l}\text { Logs in repeatedly to practice remembering and } \\
\text { entering a password. }\end{array}$ \\
\hline Personal Validation Questions & $\begin{array}{l}\text { Relies on the personal validation questions to } \\
\text { reset forgotten passwords. }\end{array}$ \\
\hline Single Sign-On & $\begin{array}{l}\text { Sometimes uses single sign-on to log into differ- } \\
\text { ent websites. }\end{array}$ \\
\hline Password Rules & $\begin{array}{l}\text { Creates passwords by consulting the available } \\
\text { password policy. }\end{array}$ \\
\hline $\begin{array}{l}\text { Relies on password resets for } \\
\text { infrequently used accounts }\end{array}$ & $\begin{array}{l}\text { Resets account passwords when accessing infre- } \\
\text { quently used websites. }\end{array}$ \\
\hline \\
\hline Guessing attack or & At login, attempts to guess own passwords. \\
\hline Dictionary attack on self & esses all of own reused passwords. \\
\hline \multicolumn{2}{|l|}{ Password Difficulties } \\
\hline $\begin{array}{l}\text { Password reuse not working } \\
\text { well }\end{array}$ & $\begin{array}{l}\text { Reuses passwords, but still has problems man- } \\
\text { aging or remembering. }\end{array}$ \\
\hline $\begin{array}{l}\text { Password reuse for memorabil- } \\
\text { ity }\end{array}$ & $\begin{array}{l}\text { Reuses passwords because unable to remember } \\
\text { more passwords. }\end{array}$ \\
\hline Unwanted accounts & $\begin{array}{l}\text { Finds self compelled to create more accounts } \\
\text { than wants or needs. }\end{array}$ \\
\hline
\end{tabular}


Table 5.1 - Continued from previous page

\begin{tabular}{|c|c|}
\hline Code Name & Description \\
\hline \multicolumn{2}{|l|}{ Security Concerns } \\
\hline Risk perception & $\begin{array}{l}\text { Assesses the risk of losing personal data when } \\
\text { creating passwords. }\end{array}$ \\
\hline Privacy & $\begin{array}{l}\text { Explicitly considers privacy when creating ac- } \\
\text { counts. }\end{array}$ \\
\hline Trust & $\begin{array}{l}\text { Considers trust in the website when creating ac- } \\
\text { counts. }\end{array}$ \\
\hline Fear/uncertainty & $\begin{array}{l}\text { Expresses fear about the consequences of pass- } \\
\text { word choices and decisions. }\end{array}$ \\
\hline Difficulty & $\begin{array}{l}\text { Expresses the difficulty of managing and re- } \\
\text { membering passwords. }\end{array}$ \\
\hline Device security & $\begin{array}{l}\text { Considers the security of the device being used } \\
\text { when creating and entering passwords. }\end{array}$ \\
\hline \multicolumn{2}{|l|}{ Behaviour Change } \\
\hline Change of habit & $\begin{array}{l}\text { Has had a major change of behaviour in how } \\
\text { they create, manage, or remember passwords. }\end{array}$ \\
\hline Hacked & $\begin{array}{l}\text { Described an incident where they found the se- } \\
\text { curity of an account had been breached. }\end{array}$ \\
\hline How to tell if hacked? & $\begin{array}{l}\text { Described uncertainty about whether or not an } \\
\text { account was secure. }\end{array}$ \\
\hline Evidence of hack & $\begin{array}{l}\text { Described concrete evidence of a security } \\
\text { breach. }\end{array}$ \\
\hline
\end{tabular}

A list of codes used in open coding is presented in Table 5.1, along with a brief explanation of each code. The groupings in the table are the result of the first round of axial coding where we collected codes with a similar focus. Following this, we identified a temporal ordering, and then assembled our groupings into larger categories following this order. These categories are described in the subsequent sections. 


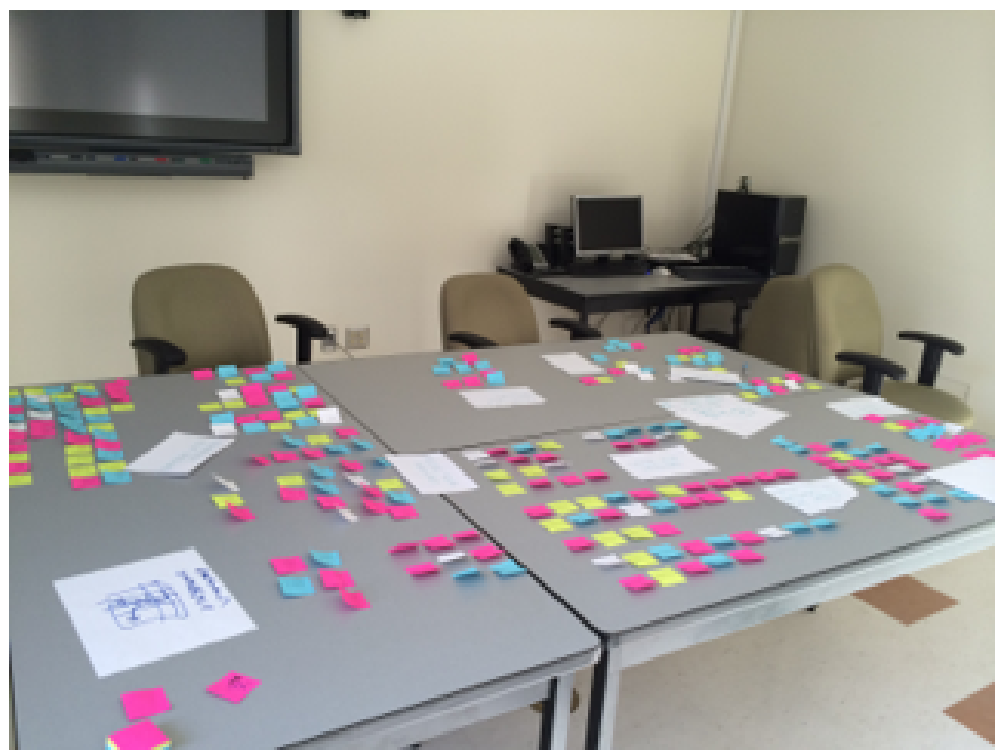

Figure 5.2: Rearranging the codes to look for patterns in the axial coding process.

\section{Choose Your Password}

At some point, every user must create their passwords and how they do this is up to them. In our interviews, participants discussed a number of strategies that they employed when choosing passwords. Some participants included personal information in their passwords. Participants mentioned including the birthdays of loved ones, phone numbers, and personal information such as hobbies in their passwords.

"I'll try to usually think of some kind of hobby of mine, uhh, whether it would be something like hockey or a video game and a video game character, and I'll try to link it to that. Something that I usually think about quite a bit." - P24

These strategies were often combined with affective strategies that included personally meaningful information in passwords. One participant told us that she had changed her passwords to a personal goal, so that the password would be easy to recall, but also so that she would be continually reminded of the goal.

"I read an article this, this, uhh, month, that said "whatever your goal is, make that your password' [okay] and you can still follow their rules ... but because you're going to be entering in your password so many times a day, make it your goal, and it can be anything, you know." - P15 
Another participant said that she included religious phrases such as "God is good" in her passwords, as a reminder of her beliefs and priorities.

Another strategy described was temporal. One participant told us that she changed email addresses depending on the point in her life (it appeared that she habitually switched all of her email into the address associated with her current educational institution). She had a password associated with each of her email addresses (when used as usernames). At login, she considered the time period in which she created the account, and entered the password linked with that email address and time of life.

A number of participants told us that they linked passwords closely to website content. As an example, one participant told us that if he was creating an account on an online store, he might incorporate the item he was purchasing into his password.

A few participants mentioned an algorithmic strategy for creating passwords. They systematically combined pieces of information to create passwords with a consistent format. Participants described different pieces of information that were included in their passwords. One participant said she included a piece of information associated with the website, as well as a piece of personal information in each password. Participants also had a few standard symbols, numbers, or words that they recombined for variation in their passwords.

External factors are also taken into account when choosing passwords. Several participants told us that they liked having the password policy rules displayed at create time, because they can factor them into their passwords, rather than having to create a password and modify it when it does not satisfy the policy.

\section{Reuse Your Password}

Password creation always happens with a new account, but users almost always have other accounts as well. Most of our participants reused passwords across accounts. We were interested in how they chose to reuse passwords across accounts, and how they matched passwords with accounts.

The participants in our study who reused passwords all built a personal model of reuse. Often, participants described categorizing their accounts and assigning passwords to categories. Participants described a number of different categorization strategies. 
Security was a common consideration that many people mentioned.

"Like I said, it depends on what the website actually is. If it requires a weak password according to me or a strong one, I'll choose it on that basis and probably alter a letter or two." - P25

Participants assessed the security needs of the websites, and they referenced matters such as privacy, and confidentiality without clarifying those terms. Many participants explained that they treat accounts differently if they store credit card information. We were unsure of how they assessed the security needs for non-financial personal information (such as on social networking websites).

Several participants described reuse strategies that hinged on password policies. One participant told us that he maintained a set of five passwords that fit increasingly complex password rules. If the password rules were easily viewed, he chose the appropriate password. If the password policy was not displayed, he began by trying the simplest password and only trying more complex passwords if the site rejected the simpler password.

Other participants described a semantic or thematic approach to their password reuse. They attempted to reuse passwords on accounts with similar purposes or contents. Examples included using the same password for social media websites, or across online shopping accounts. Participants also described strategies that organized passwords into less obvious semantic categories. These included using the same password on all professional accounts, or on all accounts that had low personal value.

Unexpectedly, many participants discussed frequency of use when describing password reuse. Participants mentioned having trouble remembering passwords for infrequentlyused accounts, but surprisingly, they often seemed to feel that infrequent use indicated a lack of need for security. Correspondingly, these same participants saw frequentlyused accounts as needing more protection. One way of bolstering the frequency of infrequently-used passwords was to have a single password for infrequently-used accounts, but it was unclear whether the purpose of this was to group accounts.

It was clear in the interviews that although many people had several reused passwords, there was a primary password that was reused on most accounts. Participants referenced this password in a variety of ways, but the language used indicated the importance of this password. One participant called it her "go-to password" and 
told us that she relied on it because she trusted the person who had chosen it for her. Several participants referred to a certain password as being "familiar" or "easy". Many participants remarked that they had many passwords that were variations on a single password, and it appeared that these were often variations on this most used password.

"[How many unique passwords do you have?] Eight? But it's always, like, you know, adding a one at the end when I forget. [So some of them are slight variations?] Yeah, yeah." - P9

A few participants were unable to describe any particular strategy to their reuse, although they definitely did reuse passwords. One participant told us that they "randomly" choose one of their reused passwords, and another participant said that they cycle through their reused passwords in order when creating accounts. None of these participants mentioned any reasoning for their habits.

\section{Commit Your Password}

After assigning a password to an account, the user must be able to keep track of this password. In our study, participants described a variety of coping strategies that they used to remember (in the active sense - store) their passwords.

Writing Passwords Down: The majority of participants told us that they wrote at least some of their passwords down. Some participants described strategies where they recorded all of their passwords, and others told us that it was a strategy that they used only in special cases. Most participants said that they wrote their passwords down to prevent forgetting them, but others wrote their passwords down as part of a larger strategy. One participant told us that she records her passwords in a spreadsheet for her husband to access in case of emergency. She later implied that she sometimes consults the spreadsheet for herself, but this is not the primary reason for keeping it.

"[Do you ever write your passwords down?] Only maybe a couple of banking ones, they're the only ones, my banking, so if I die my husband can find them." - P5

Most participants appeared to view their password recording as a backup strategy 
rather than a constant resource. Interestingly too, writing passwords down can support not needing to rely on such techniques forever. One participant told us that she wrote passwords down only until she had memorized them. She also used a rehearsal strategy to help her memorize her passwords.

"If it's new, I'll write it down for the first couple of times, but if it's new, I'll try to remember it, try to memorize it. I'll log in a bunch of times until I've memorized it." - P11

A number of participants described special cases where they would write passwords down. These special cases included assigned passwords, websites without any backup mechanisms (such as online password resets), and websites where the use of cookies is disabled. Other participants told us that they recorded hints or clues to their passwords. A few participants told us that they recorded usernames, either with or without the corresponding password. Another strategy was to write down only part of the password or some other form of password hint.

"I email them to myself, and I have a folder with all my passwords in it [This is an email folder?], (nods) and instead of putting the actual password in there, I put something to remind me what the password was." $-\mathrm{P} 13$

An important consideration in the safety of recording passwords is how they are stored. Participants in our study described a variety of storage strategies for recorded passwords. Some participants wrote down their passwords on physical media, such as post-it notes, or journals. Often, they chose places near their computers to keep these passwords. One participant told us she pins a list of passwords to her bulletin board, while another described writing her passwords on a box kept on her desk. Some participants went to lengths to hide these passwords, by keeping the post-its underneath keyboards or by carrying the list of passwords with them, but others seemed unconcerned. Participants also shared a variety of strategies for storing their passwords digitally. These strategies included dedicated password documents (Excel spreadsheets, or Word documents), passwords emailed to themselves, passwords kept in online notebooks (such as Evernote), and passwords stored in desktop widgets.

The accessibility of recorded passwords was a key issue. For participants who chose to store their passwords digitally, they often mentioned concerns about having the 
password list when it was needed. A few participants mentioned using services like Dropbox to keep their password lists in the cloud, and participants who emailed passwords to themselves appeared to have chosen this technique for accessibility. One participant emphasized the need for accessibility when he described using services that synced across devices to store passwords.

"[Do you ever write your passwords down?] On my phone, sometimes. ... Usually you would either keep it in, like, Google Keep, or iCloud, or messenger, or Evernote. In my case, Evernote. I use a lot of Evernote, so.... Anything that is really sync-able to multiple devices that way it is easier for me to store info." - P16

Password Managers: Almost all of our participants reported using the password managers built into web browsers. A few participants told us that they only used these tools in specific contexts: two participants told us that they saved their passwords in the browser at work, but not at home, and many participants clarified that they only saved passwords on their own computers. A number of participants also clarified that they only saved passwords for certain accounts in the password manager. Most commonly, participants said that they did not save the passwords for banking websites, but a few people specified that they did not save passwords on any websites that required credit card details.

One participant in our study told us that he used to use a dedicated password manager, but had stopped using it. He was vague on the details of the password manager, and could not remember its name, but was able to tell us that he had stopped using it because it was inconvenient to have to copy passwords out of the password manager.

"I've used, umm, I can't recall the name, but I've used one before in the past. [What made you start or stop using it?] It was just inconvenient because it encrypted it every time and I'd have to decrypt it. [Oh, okay] It was the same issue, it's like encrypting and decrypting constantly. It's just more convenient to have it. [And was that kind of like the time that it took to...] Yeah, but you had to basically sorta copy it, paste it into a search box. It wasn't the time issue, it was just the hassle if I wanted to do it with multiple passwords." - P25

This participant refers to the time and hassle of encryption and decryption, but we 
Millions of products that make you smile

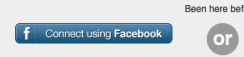

Figure 5.3: A detail of one of the websites used as a prop in our interviews.

speculate that he is referring to the process of hashing a master password.

Many websites offer the user the opportunity to remain logged into a page via a cookie saved in the browser. This choice is often presented at login, via a checkbox that says "Remember me", "Stay signed in", or something similar. Although this mechanism is not one that saves passwords, it accomplishes the same result by removing the need for the user to enter a password. In our interviews, we asked about cookies directly after asking about browser-based password managers, and it was clear that a number of participants did not understand the difference between the mechanisms.

"YYou know how sometimes you'll go to log in, and there will be a box for your username, a box for your password, and then there'll be a little box to tick that says "Remember me"? Do you ever tick that box?] Yeah. Because that's the same as saying "save your password', right?"

- P16

Some participants appeared to treat the mechanisms identically (typically, relying on both), but others told us that they used one or the other without giving much justification for their habits.

Other Tools: Over the course of the interviews, participants mentioned a few other tools and techniques that they used to keep track of passwords. These tools included a Smartwallet app, and single sign-on. Although a single sign-on option ("Connect via Facebook" - see Figure 5.3) was prominently displayed on the password creation prop card, only two participants commented on it. One participant told us she would use the option when it was available because she had a hard time remembering even her reused passwords. However, another participant (quoted in Section 5.4.1) said she would not use it, because she did not want any extra information cluttering her Facebook page. It is difficult to know why other participants did not mention any kind of single sign-on, since the cue was equally visible to all participants. Our interview script did not prompt them to specifically look at the Facebook button, but they were 
told to imagine they were on that page. Possibly, this indicates that most users do not understand how single sign-on services can be used as an alternative to reusing passwords.

In summary, it was clear that a variety of tools and strategies were helping participants cope with their passwords. Participants saved passwords in browser-based password managers to handle the case when they were using their own computers, but needed backup mechanisms for times when using other computers.

\section{Forget Your Password}

After a user has memorized their password, there is always the chance they may forget it. Users have many passwords, and it is clear that handling forgotten passwords is a large part of the password management task.

Several participants described situations where they could not remember their passwords at login, and told us their first action would be to try and guess their password. Some participants described a kind of targeted dictionary attack on themselves, where they would guess all of their reused passwords.

"Sometimes, I do forget, but I try everything else [all of the passwords]" - P17

Other participants described guessing strategies where they attempted to recreate their motivation for being on the site (for example, the item they were buying when they created a shopping account), or the password they would have been likely to pick in the time period they created the account. Still others said they would try to recreate algorithms for password creation, or look at the password policy to make a better guess at their own password.

The other fallback strategy that participants described was the password reset mechanism. In this, we include both personal verification questions and email resets. Almost all participants told us that they have reset forgotten passwords, and it appears that many users do this on a regular basis. Some users seem fine with this as a strategy, but others raised objections. One participant told us that she had begun writing her passwords down when she realized she was resetting her passwords too often. Another participant remarked that she had not considered how often she reset 
her passwords until the interview, but that it was a major part of her password coping strategy:

"It's funny, I never really thought about it, but I guess I do that a fair amount." - P19

\section{Live with Your Passwords}

Passwords and accounts can last a long time: once passwords have been created and linked to accounts, all users must begin the long process of living with and coping with their passwords.

A number of participants in our study commented on the difficulty of managing and remembering passwords. Participants displayed different attitudes toward this difficulty. One participant referred to passwords as agonizing.

"Then what do I do? Ohhh, my gawd. Then I agonize for a few minutes." $\mathrm{P} 13$

Another participant seemed resigned to reusing passwords.

"[Do you ever reuse passwords?] Oh yeah! (laughs)" - P9

Participants also referred to fears of doing the wrong thing, and uncertainty about the outcomes of password decisions. Some participants explicitly referred to privacy, but only a few referred to private information that was not financial.

As time progresses, a user may change their behaviour in some way. In our study, a number of participants described changes in behaviour that had occurred for a variety of reasons. Some users described stopping or starting using tools. One participant told us that she no longer saved passwords in the browser because she had heard that this behaviour could be dangerous.

"I used to and then I took one of these studies and they helped me with understanding why that was not a good idea. [Did you at that point go and erase the ones that were already in there?] Yes." - P13

Another participant told us that he used to use a password manager, but had stopped using it because of the time it took to copy and paste passwords when using the 
password manager. He describes the hassle of using the password manager in a quote in Section 5.4.2.

One reason to change an existing password is in the case of a security breach. A number of participants in our study described situations where they had changed account passwords after suspecting that an account was not secure. Examples of breached accounts included email and PayPal accounts. As participants described their security breaches, it emerged that they were often unsure about whether they had been attacked, and sometimes had to made decisions without really knowing what had happened.

"At least, I think that I've been hacked. [What kind of clues, what would be a kind of signal to you? Has it ever happened to you?] It has, because my friends told me they got these really strange emails, from my email, supposedly sent from me, that were obviously ads for something or other and they were like 'hey, this doesn't sound like you'." - P19

A few participants brought up changing their passwords in the case of more minor suspected security breaches. One participant told us that they changed their passwords when they thought a friend might have seen their list of passwords, and another participant said they had changed their Facebook password when they thought they might have left the account logged in on a friend's computer.

\subsubsection{Selective Coding}

The last coding step in Grounded Theory is selective coding, where the researchers attempt to identify a unifying core code that describes the underlying phenomenon in the observed and interpreted behaviour.

As we analyzed the data, a central theme about rationing and budgeting began to emerge. In all phases, our participants described ways in which they stretched thin resources: memorization, attention, creativity, and security knowledge. Similar to the way in which we ration and conserve time, energy, food, and money, participants were handling password management by devoting appropriate resources to accounts of great importance, and then devoting less energy to other accounts, and generalizing their approach to similar accounts to save effort. We do not suggest that this budget 
is necessarily fixed, but rather that users' efforts, attention and memory are limited and users must be thoughtful in their commitments. In their work on organizational security, Beautement et al. [9] suggest that organizations need to budget for the costs (both time and money) of organizational compliance. Our suggestion about rationing differs in that we identify that individual users are budgeting their own time and effort. We are not suggesting this arises because of a lack of willingness to comply, but rather from a paucity of cognitive resources.

In the following sections, we systematically examine how rationing plays a role in each of the themes of axial coding.

\section{Choose Your Password}

When choosing their passwords, participants rationed their efforts in a variety of ways. For participants with formulaic or algorithmic strategies, part of their investment was in memorizing their personalized strategy. By remembering that their strategy is to include a word related to the website, they reduced the amount of effort that it takes to choose a password on a new website. Participants who closely associated their passwords with a username were engaging in a similar strategy. Remembering usernames can be difficult, but if you have a consistent strategy to associate a password with a username, effort can be more effectively rationed to each account.

The interviews asked participants what they would do when creating an account if their password was rejected on the grounds of insufficient complexity (for example, lacking a symbol). Most participants reported that their strategy in this situation was to append a symbol to the password. Most participants referenced "their" symbol, and told us that they had a habitual symbol that they used in this situation. This coping strategy implies a way of rationing effort across situations that cannot be predicted. If participants knew their password would need a symbol, they would have begun with a symbol. But since they are unable to see the password policy, they have developed sensible coping strategies that conserve memory and effort in these situations.

Many participants told us that they reused pieces of passwords in a variety of ways. Many participants referenced appending different endings onto the same widely reused passwords. Participants also discussed using this strategy as part of their password 
changes; one participant told us that after he realized a friend might have got access to his list of recorded passwords, he had changed his passwords, but had simply added characters to the existing passwords.

"Like I said, I have them all stored on a text file, right. And once, a friend of mine or an acquaintance borrowed that USB drive, and I felt that he would have access to everything so I went and changed everything. [Okay] But all I did was add like a letter or two." - P25

\section{Reuse Your Password}

One main way in which users ration their efforts is in not choosing a new password for every account. Reusing passwords allows users to conserve energy across their large number of accounts.

As participants discussed how they would create a password for a new account on an online shopping website, many digressed into a discussion about accounts that do not matter to them. One participant told us that she had a password that she used on accounts where she would not care if she was hacked. Another participant referenced having a password that she would not mind sharing with others. Whether or not these participants actually would not care if others had access to their account, their behaviour shows that they are rationing the effort they put into these accounts by the amount of effort they merit.

An important aspect of rationing is that those who need more should get more, and we found evidence that users were applying this principle in their password management strategies. Many participants referenced special habits for their online banking: participants told us that they did not reuse their banking passwords, that they would not log into their bank on a shared computer, and that they would not enable cookies or save their banking login in the password manager. All of these behaviours indicate that users are willing to ration more effort into accounts that they perceive as needing it.

Participants referenced using different behaviour in different contexts. Two participants referenced a different set of habits for their work passwords, and both implied that they were less careful about security for these passwords. It was difficult to know if this was because the information was seen as less personal, or because they 
felt that the physical environment was more secure, or if it was simply because they used those accounts more frequently, but they were assessing information about the context of use and rationing effort differently to it.

\section{Commit Your Password}

Memorizing passwords is one of the most difficult parts of the password management task for users. Passwords must be maintained over long periods of time, with sporadic and unforeseeable usage patterns. Using tools such as password managers and techniques to write passwords down is how users allot effort into the unknown needs of the future.

Availability and accessibility are key issues for password managers. Managers are often only available on personal computers. Dedicated password manager software sometimes have associated smartphone apps that allow users to take their passwords away, but the browser-based managers are largely only tied to one computer. This means that users must consider the effort they put into making sure their passwords are available to them when they need them.

Participants in our study described using a combination of techniques to keep track of their passwords. Some participants were heavily invested in one strategy, but most participants appeared to know a few of their passwords, to have some of them written down, and to have some of them stored in a password manager. This strategy seems to ration effort across time and place - when at home, the password manager saves the passwords for almost all of their accounts, or they might have easy access to the recorded passwords. When elsewhere, they cope by remembering their passwords, or by carrying some of the passwords with them. It appeared that some participants were sacrificing convenience for security in these situations, which indicated another aspect of rationing in their coping strategies.

\section{Forget Your Password}

All of the participants in our study told us that they have reset passwords when they are forgotten. Participants clearly regarded this as being separate from a change of password, which seems to indicate that forgotten passwords are seen as part of the 
landscape of password management. Users expect to have to handle a loss of memory, or a failure of a coping strategy. It appears that the password reset mechanism allows users some flexibility in their rationing strategy. In particular, many participants appeared to rely on email resets. In situations where a password reset was not available, participants described allocating extra effort to the situation, often to ensure that the password was recorded.

In the situation where they were resetting a password, many participants told us that they would reuse an existing password, or change the password to what they thought it had been or what it should have been. It appeared that their coping strategies were equipped to handle these situations without any particular sense of frustration or personal loss.

\section{Live with Your Password}

Throughout the interviews, we heard a number of remarks on the difficulty of password management. Although participants are resigned to the realities of passwords, they still present difficulties. Finding and implementing coping strategies for the difficulties of passwords involved effort and unpleasantness. Similarly, rationing itself is a difficult task! The decisions about how to allot time, energy, and effort are not always obvious to users. Users referred to missing information that would have made it easier to cope: unseen password policies, misunderstood security requirements, and invisible security breaches.

\subsubsection{The Password Life Cycle}

In the final step of Grounded Theory, we look back at the identified codes, patterns, and relationships in order to form them into a theory.

Our theory is that there is a password life cycle - a progression of stages through which every password passes. Passwords are created, assigned to an account, then recorded or memorized, lived with, and then potentially forgotten. Old passwords are then reused or adapted in the creation of new passwords, and the cycle continues.

Figure 5.4 illustrates the stages of the password cycle. The cycle begins when the user 


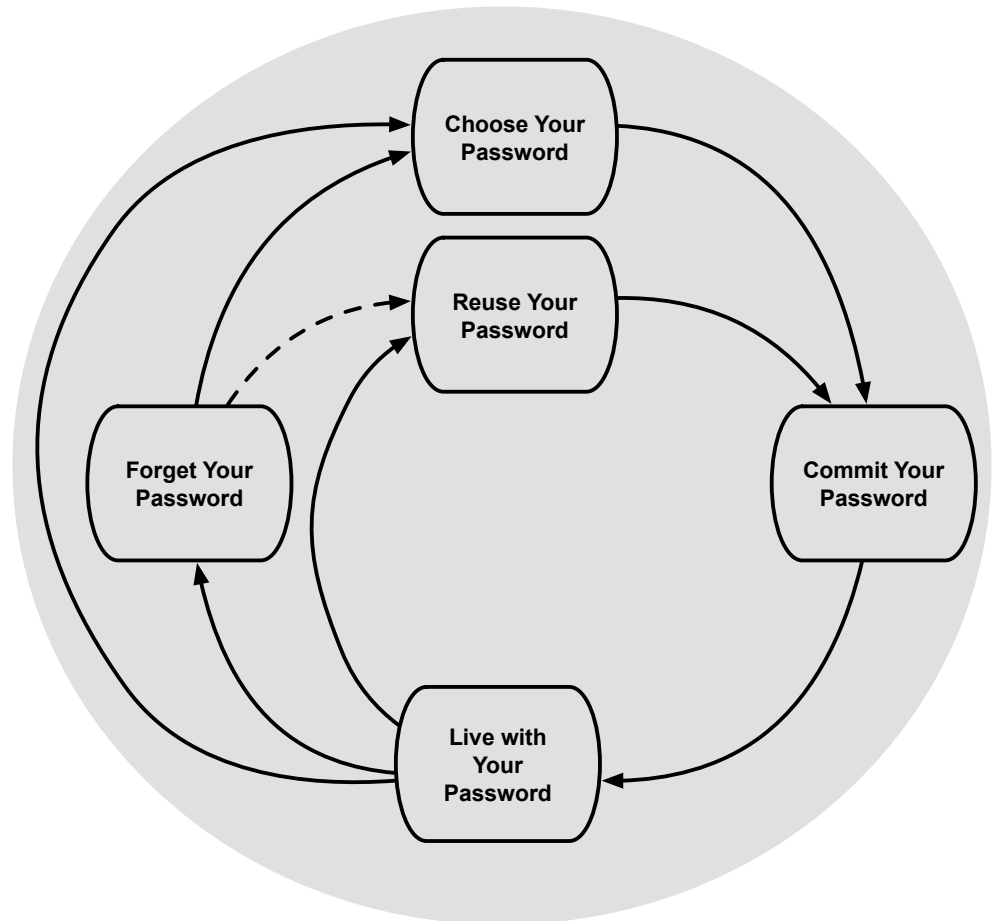

Figure 5.4: The password life cycle.

needs to create a password for a new account. Theoretically, a user might begin with no passwords at all, and have to fabricate one from scratch, but they may also have existing strategies and password phrases that they will integrate into a new password. This password must next be committed, either memorized or recorded, so that it can be later used for login. Assuming the commitment process is successful, the user then lives with their password. They login and access their accounts successfully. If they successfully remember their password, and it is appropriate for reuse, they can then reuse that password. If the password must be changed (because it is forgotten, because someone else has learned it, or because of enforced password change policies), they must return to password creation.

Rationing is present at every step of the password life cycle. Users ration effort at creating new passwords, they reuse passwords to put more protection on the most valued accounts, they reduce the effort of memorization by saving passwords in managers or by writing them down, and they strategically budget the attention they pay to passwords on existing accounts. Users save resources from inconsequential accounts so that they can devote them to more important accounts. Allotting time, attention, and energy to different accounts forms the backbone of users' coping strategies. As 
with other forms of rationing, users may scrimp on effort for some accounts to save it for others.

Rationing contributes to the cycle of password reuse. As effort is reduced from some accounts, it is saved for new ones. Reused passwords are handed down from existing accounts, saving the user the time and energy of creating and memorizing a new password.

\subsection{Life Cycle Example}

In this section, we provide an example of how a user's passwords might look in various stages of their life cycles. The example (shown in Table 5.2) shows accounts across the top and events down the side. The passwords for each event are shown in the cells.

This example is not meant to be representative, and does not express the true complexity of a real user's passwords. It is intended to illustrate how the life cycle might play out for an example user, and to demonstrate different aspects of the life cycle. Although the example is fictitious, the behaviours reflect what was described to us in the interviews.

In the example, we see the user begin with one password, fluffy, that becomes her go-to password. She creates new accounts, often reusing her go-to password, and varying it to accommodate various password policies. She has a consistent strategy for varying passwords - she tries to use a question mark when a symbol is required and a 2 when a digit is needed. For her bank, she has made an effort to use a different password than on her other accounts. However, to make it memorable, she has used the same symbol and digit as usual. When the bank requires a password change, she increments her usual digit by one, but later forgets the changed password. She reverts back to her usual digit, but places it at the beginning of the password instead of at the end. After some time, the user realizes that her email account is sending spam. She decides to change the password, but not knowing what to change the password to, decides to reuse the root of her bank password (Apple). Although the old email password was reused extensively, she decides that it would be too much trouble to change it everywhere, and decides only to change her email password. 


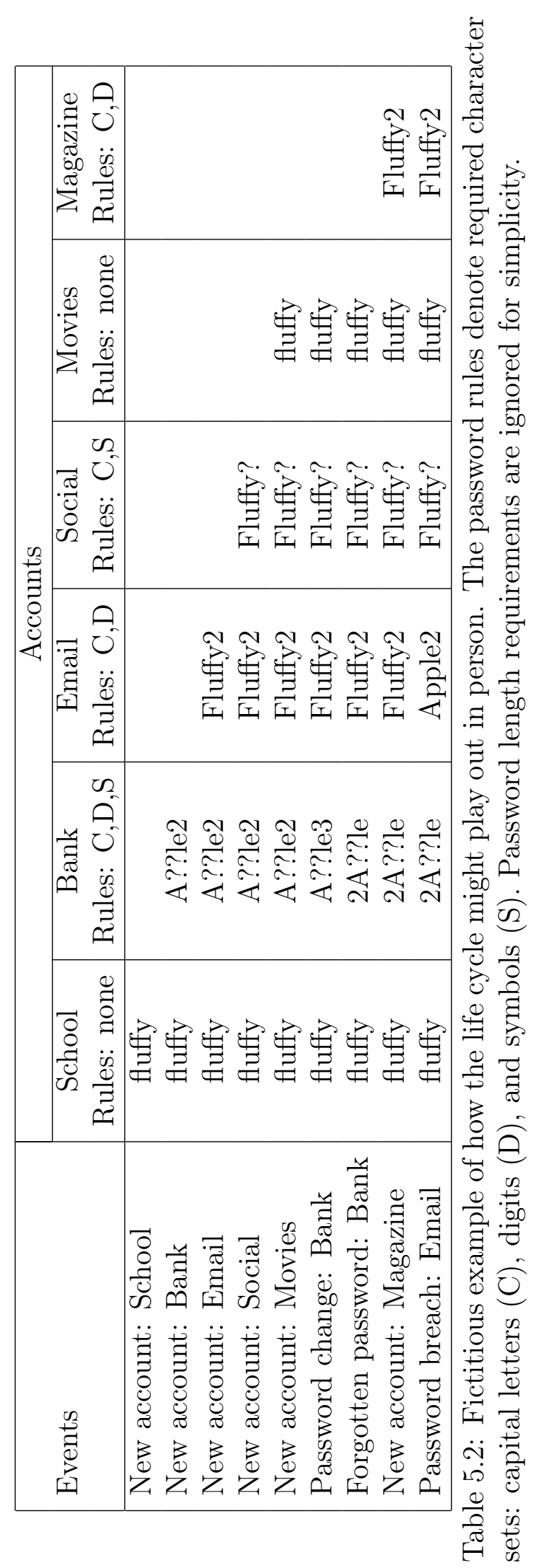


The table demonstrates the life cycle in a few ways. The example user has trouble creating passwords, and often chooses to reuse a password rather than creating a new one. She has chosen to ration her effort differently for different accounts, and when she has to create a bank password she opts for a new password. However, when obliged to change her bank password, she is unsuccessful at committing it to memory, and chooses to revert to a more memorable variation of the original password. Another time, she finds that her email has been compromised, and must change the password to secure the account. But for her other accounts, she is living with her passwords and generally does not have extensive problems.

The fluffy password gives a concrete example of the cycle. It is created for the school account, reused directly on the movies account, and adapted for reuse on the email, social, and magazine accounts, which have different password rules. The user later forgets the adaptation made for the email account and changes the password for that account, abandoning the fluffy password.

\subsection{Discussion}

Our theory suggests a number of ways in which the design of security products could better support users.

\subsubsection{Writing Passwords Down}

While writing passwords down is an intuitive and reasonable way of handling security, users need helpful guidance on the right way to store these passwords. Writing passwords down is conventionally understood to be insecure, but many security experts actually advocate writing passwords down $[25,115]$ if passwords can be kept in a physically secure location. Many users do write their passwords down, but the caveat about storage is poorly understood. In our study, participants reported keeping their password lists in their email, in Dropbox, on their cell phones, or saved on their computer desktops. Recording passwords is a sensible way of conserving cognitive load, and users should be encouraged to make the small changes that could make this habit safer. 
To address the storage problem and guide users to safer password storage, a plausible solution might be the development of a service specifically to securely store passwords. Password storage notebooks do exist (e.g., The Personal Internet Address \& Password Log Book [104]), but there is no equivalent online service. In the absence of a trustworthy electronic service, it seems possible to better emphasize the notion of physical security of stored passwords to users, and suggest secure and sensible places to keep lists of passwords, such as in a locked desk drawer or a safety deposit box.

\subsubsection{Password Cues}

Another finding of our study is that participants often had trouble matching passwords to usernames or to websites. If users were able to better match their passwords to websites, they would not have to resort to strategies where they reveal multiple passwords to potential attackers by trying all of them at login. Although some users currently try to create a cued match from password to website by including websiterelated information in their passwords, these strategies can place an additional burden on the user because they must then remember the cueing strategy in addition to the cued password. Reducing this burden might allow users to transfer effort into other aspects of the password task.

We suggest that explicit image cues could help users better associate passwords with accounts. Websites could be designed to associate these cues with usernames and present them at password creation and at every login. Since the cues would have no relation to the password, it would not be necessary for them to be secret. This could be similar to the image-based anti-phishing mechanisms found on some financial websites (for example, Bank of America's SiteKey [8]), but rather than serving to authenticate the website to the user, the cues would create an explicit link to help users associate passwords with websites.

\subsubsection{Password Managers}

We were surprised to find that none of our participants used a dedicated password manager, and also surprised that not everyone was using the browser-based password 
managers. As long as they are well-designed, and do not store passwords in the clear, password managers seem to offer one of the best solutions for password management: comprehensive, convenient, and safe. However, most of our participants appeared unaware of prominent password managers, and some participants expressed distrust in this software. We suggest that the better integration of password managers into operating systems and browsers would help with both visibility and trust. A few participants in our study did mention having passwords saved in Apple's iCloud Keychain, although it wasn't clear that any of the users were taking advantage of the password creation mechanism, or the cross-device capabilities.

We speculate that password manager software could be improved to integrate password cues and to facilitate reuse. We know that not all websites will want to integrate these kinds of features into their existing mechanisms, and the advantage of a password manager is that it is controlled by the user, and does not require changes to existing websites. A well-integrated and usable password manager would let users ration effort into a mechanism that genuinely kept them safer.

\subsubsection{Single Sign-On}

Although the use of single sign-on would address many of users' password problems, the participants in our study appeared either unaware of or ill-informed about single sign-on. Although a single sign-on "button" was equally visible to all participants in our interviews (on the website screenshot), only two commented on it at all. One said she used it sometimes because she had trouble remembering even her reused passwords, but another participant explained that she would not use it because she did not want information cluttering up her Facebook page.

Her explanation showed a strong misconception about how single sign-on works. Instead of understanding that Facebook's role in single sign-on is to verify your identity, she thought that she was signing into Facebook and using the online store as a part of Facebook. This indicates a need for independent single sign-on providers, who do not have a stake in personal information. The biggest current providers of single sign-on are Google and Facebook, both of whom have other reasons to be interested in browsing and usage patterns. Although users do not completely understand how 
single sign-on works, they do not want their activities to be visible to uninvolved parties. A better option for single sign-on providers would be independent entities who only verified authentication attempts, similar to the certificate authorities who currently issue SSL certificates.

Addressing the issues with existing single sign-on services would allow users to take advantage of these services, and allow them to better ration and conserve their password efforts. Single sign-on gives the user the advantages of reusing passwords (i.e., having to remember fewer passwords) without the risk, and allows them to harness existing strategies while remaining more secure.

\subsubsection{Extra Information}

When discussing information that participants look for when creating passwords, a few participants mentioned password strength meters and a number of participants brought up the subject of password policies. Participants wanted to know password rules before picking a password so that they didn't have to waste effort creating the "wrong" password, and wanted the additional guidance of a password strength meter. Providing strength meters and making password rules available before they are broken are minimal efforts that could simplify users' password experiences.

Throughout the discussion, but particularly when they were discussing security breaches, participants referenced a lack of information about their passwords and accounts. When discussing suspected hacks, they expressed uncertainty about whether they had been hacked, and an absence of information to turn to. This lack of feedback is an inherent characteristic of security because helpful information cannot be made available to attackers [146], but we still think that more information could be made available to users. Most websites log information about sign-ins and actions, and this information could be made available to the user. Obviously, this information could not assist in attacks where the attacker gains complete control of the account and the user is completely locked out, but in many cases, participants still had access to their accounts. Participants described being able to change their passwords and could have used a resource to help them find additional information about account usage. 
Having to search for clues about malicious usage is yet another security task that consumes users' time and resources. Making usage information readily available would allow users to sensibly ration and conserve their efforts when handling compromised accounts or passwords.

\subsubsection{Threat models}

One of the emergent themes during the interviews was confusion about threat models and the nature of the threat. Although worried about security, participants seemed unclear about the type of threats that concerned them. They did not differentiate between targeted personal attacks, anonymous large-scale password hacks, and the loss of private data, although they referenced all three during the discussions. Correspondingly, participants did not seem to appreciate that the defences for different attacks might vary based on the nature of the account in question. For instance, the password protecting an email account from an online attack addresses a different threat model than the unlock code stopping strangers from reading the email a mobile device. This lack of understanding has an impact on how users ration their password efforts. If they are confused about the type of threat, they may misdirect their efforts, leaving valued accounts unprotected and over-protecting less vulnerable accounts.

Understanding of threat models informs how users categorize their accounts for reuse. In order to reuse passwords safely, users must be able to better assess their security needs on websites. If users are going to reuse passwords, either by themselves or with support from a password manager, it is important that they understand the consequences. For example, it is probably unwise for users to reuse passwords from high-importance accounts on low-importance accounts.

Assessing the threat models is important, but users are also trading off their time. Herley [64] points out that if users were to follow all given advice, the security benefits would be swamped by the time spent following the advice. He proposes instead an economic model where both threats and benefits are assessed. In order to consider

both threats and benefits, users need to be able to reason about the severity and likelihood of threats, and to consider carefully benefits such as uninterrupted routines and time that can be devoted to primary tasks. 


\subsection{Conclusion}

In this chapter, we investigated how users cope with the difficulties of living with passwords. We conducted interviews, and performed analysis using the Grounded Theory methodology. We found that users have complex coping strategies that combine a variety of tactics. They ration effort to devote resources to accounts they feel of greater importance and minimize their effort for accounts of lesser importance. Over time, this leads to a life cycle of password usage whereby passwords are developed, reused, adapted, discarded, or forgotten.

We suggest that our findings indicate new opportunities for better supporting users. For example, password managers might be designed to facilitate safe reuse. A proactive alternative to strength meters could help users pick appropriate passwords for each account. Accounts could help users by providing cues and password rules to help users link passwords with accounts. We suggest that the design of password systems and security technologies should incorporate the implications of the password life cycle to better support users.

Of course, we acknowledge that there can be dangerous security implications to password reuse and other coping strategies described here. Reusing whole passwords or pieces of passwords can make it easier for an attacker to gain access to multiple accounts once the password for one is guessed. Recorded passwords can be lost or stolen. However, these kinds of threats are personal, and many attacks are impersonal. Often, attackers want access to an email account, not a particular email account.

The contribution of this work is the identification of important patterns underlying user coping strategies. Users are not stubbornly refusing to follow password advice, they are instead carefully managing their resources to cope with impossible demands. Their solutions are often flawed, but they deserve consideration and may indicate better strategies for security. In choosing where to build roads, it may be best to pave the paths that users already walk. 


\section{Chapter 6}

\section{Expert Password Management}

\subsection{Introduction}

Following on our investigation of end users' password management techniques and coping strategies (Chapter 5), we became interested in the behaviour of expert and power users. How are the practices of those who are knowledgeable about computer security different from or similar to those of non-experts? We conducted a series of interviews with graduate students and researchers in computer security, asking them about their password management behaviour. We found that these knowledgeable users described a dichotomy of behaviour where they employed more secure behaviour on important accounts that they deemed more worthy, but employed similar practices to non-expert users on their remaining accounts.

Little work exists on the security habits of experts, who must be affected by the same problems that affect all users: difficulties choosing random passwords, difficulties remembering passwords, and multitudinous accounts. If remembering large numbers of random passwords is difficult or near-impossible for non-expert users, it should be similarly difficult for experts.

The goal of our interviews was to better understand the practices of expert users, and to see how they address the demands of creating and managing large numbers of passwords. Do experts rely on similar coping strategies as non-experts? What kind of tools and techniques do they use? We hoped to find insight from the practices and 
coping strategies of experts that will help us form recommendations for non-experts.

In the following sections, we describe our study methodology and present our results. As in Chapter 5, the interviews yielded a set of descriptive quantitative data as well as a richer qualitative data set. We first analyze the data independently before comparing it to the data from the earlier interviews. We identify similarities and differences, and use these to recommend ways in which the behaviour of experts can be used to help form recommendations for non-expert users. We also identify areas of difficulty: password management problems that even expert knowledge cannot solve.

\subsection{Study}

The study followed the same procedure as the study in Chapter 5. Participants were interviewed about a variety of subjects relating to password management, including creating, reusing, remembering, changing, and forgetting passwords. The interviews were conducted by the researcher, who asked questions and recorded responses. The interviews were also audio-recorded to facilitate further note-taking.

The interview had two parts: a short self-administered demographics questionnaire, and the password interview. Since we were interested in the same kind of coping strategies as we explored in the life cycle work, we used the same interview script (Appendix B) and demographics questionnaire. Once again, the study was approved by the Carleton University Research Ethics Board. We emphasized to participants that they should not share their passwords with us, and that all interview questions were optional. The interviews took approximately 30 minutes, and participants were not paid.

Once again, we chose our methodology so that participants could reflect on and discuss not only what they do, but why they do it. We asked participants to elaborate on incomplete answers and to pursue alternative discussion paths that revealed their password management strategies.

We interviewed 11 expert users, primarily recruited from among the information security research groups in the Computer Science Department at ETH Zürich, but also from the community of security practitioners in Zürich. Reflecting the gender 
distribution of the security community, the majority of our participants were male (9 participants). Participants ranged in age from 24 to 35, with a median age of 28. All participants except one had a graduate degree in computer security and all were employed either as graduate students or researchers in some aspect of information security.

The purpose of this study was to add a new perspective to the interview data and analysis conducted in Chapter 5. In qualitative analysis, triangulation is the process of adding additional information and perspective to the analysis. The purpose is not to confirm the findings, but to add additional viewpoints. We analyzed our data by going through an informal open coding strategy, reusing many of the codes from the life cycle study, and adding new ones as needed. We then synthesized those findings into the five themes of axial coding from Chapter 5. Since the coding process was very similar to that in Chapter 5 , we simply present the categorized findings here.

\subsection{Results}

As in the non-expert study, the interviews yielded two datasets: a set of quantitative data describing participants' specific responses to the questions, and a qualitative dataset from their detailed responses and discussion. We first present an overview of the results using the quantitative data, and then present more detailed analysis in the qualitative results section.

\subsubsection{Results Overview}

Participants in this study had a median of 52 accounts, and reported that in an average week, they use a median of eight accounts. They reported a wide range in the number of unique passwords, from 4 to 77, with a median of 20. All but one participant reported that they sometimes or frequently reuse passwords, but most participants described a principled reuse strategy that we explore in more detail in the next section. Of those participants who reported reusing passwords, all said they reuse multiple passwords. The median number of reused passwords was three. 
Participants reported that they enter their passwords on a variety of device types, including smartphones, tablets, laptops and desktops, but most said that they did not consider the entry device when they were creating passwords. Two participants mentioned shortening their passwords, or avoiding special characters when they knew they would be using passwords on a smartphone. One participant said that when using a regular keyboard, they tried to create their passwords so that all characters requiring the use of the "shift" key were next to each other.

Slightly more than half of our participants (6 out of 11) reported that they will enter their passwords on computers belonging to friends or family members, but most qualified the statement by mentioning that they would only log into certain accounts on other people's computers. Those who said that they would not enter their passwords on computers not belonging to them said that this was a deliberate and strict policy for them.

We asked participants about how they stored passwords, and nine participants reported storing their passwords in a computer program. Of these, four participants reported using a dedicated password manager, and the rest reported storing their passwords in a web browser. Five participants told us that they wrote their passwords down, and all of these specified that writing their passwords down was something they did rarely, and only when unavoidable (e.g., in the case of an assigned password that they could not remember).

\subsubsection{Qualitative Observations}

The interview process was easier to conduct with the expert users, who were able to speak knowledgeably and fluently about their password management and security strategies. Unlike the non-expert users in the first round of interviewing, these participants were familiar with what they do to address security and often anticipated subsequent questions. While this familiarity is no doubt due to the fact that these participants spend large amounts of their lives considering security, it also seemed to highlight the a priori nature of the expert approach. These participants referenced specific policies, and as in the following quote, were emphatic about avoiding certain situations. 
[Do you ever enter your passwords on computers that don't belong to you?] No. This is something I really try to avoid. - E08

\section{Choose Your Password}

Expert participants were less forthcoming about their exact password creation strategies, but they did mention a variety of techniques. One participant had an elaborate password-generation algorithm to create their passwords. Their technique included a component related to the website, a random seed, and a personal evaluation of the required security level of the website. Several participants said that they relied on their password manager to generate passwords for accounts, but others said that they did not use this functionality for all accounts.

Although participants did not discuss the exact components of their passwords, most participants said that their passwords were rarely rejected for failing to comply with password policies, indicating that these experts were including special characters, digits, and capital letters in their passwords.

\section{Reuse Your Password}

Although password reuse is a technique often criticized by security experts, all but one of our participants said that they reused passwords on at least some of their accounts. However, most people described a careful strategy for reuse. Participants often mentioned they did not reuse all of their passwords, but that they had one or two passwords that they consistently reused for "throwaway" accounts. Participants mentioned reusing specific passwords for specific purposes, such as single-use websites, or seldom-visited websites.

[Do you reuse multiple passwords?] Yep. [How many?] Four. Four different ones that have different behaviour in terms of complying to bullshit regulations like numbers, or punctuation, or ... - E03

Conversely, participants also described restricting password reuse for other accounts.

What I perceive as important, which is typically the four or five accounts that I use on a very regular basis, I use unique passwords for all of them. 
And I believe that these passwords are strong. But on the other hand, I use a common password for ... a lot of services that badger you to create an account at times. - E10

When discussing the kind of password that they reused, participants were clear that they had "their" password, often naming it (e.g., "my easy-to-steal password" - E09). Multiple participants referenced having had their password since they began using computers. One mentioned having had their password since high school.

I would use the password that I've used since high school, that is not at all secure in any meaningful way, and I've used it for hundreds of times, for different whatever, anytime anyone asked for a password. - E03

\section{Commit Your Password}

About half of the participants said that they wrote some passwords down, and all of these described it as a kind of backup strategy. One participant said he wrote down passwords that were difficult or impossible to change. Another said that when he was issued assigned passwords, he often kept the piece of paper that came with the password. One participant said that he wrote down most of his passwords, but was explicit about how his strategy was intended as a backup strategy for infrequentlyused accounts.

I just keep them written down just in case, and there are those more throwaway accounts that I use once every ... a few times a year, but I need then to check. - E04

Some participants described writing down other pieces of information as a backup strategy. One participant who had an algorithmic password generation strategy said that he sometimes wrote down the year that he had created the password for a specific account. Together with his memorized algorithm, this small piece of information was sufficient for him to regenerate the passwords.

All but one participant described using some kind of password manager. Four participants told us they used dedicated password managers, and ten participants reported using browser-based managers. Most participants mentioned using more than one tool, and even users of dedicated password managers reported using them alongside the browser-based managers. 
Several participants described using a combination of strategies. In particular, multiple participants mentioned using password reuse in combination with password managers. One participant said that he used a password manager to randomly generate and remember passwords for important accounts, but that he opted to reuse passwords instead of storing them in the password manager for insignificant accounts.

I don't store everything in a password manager. [Why not?] Because I, I dunno, because that's kind of incon-[breaks off] -it's just another layer of inconvenience to use a password manager, and I, for me personally, it's not worth the investment to store it there. And it also kind of clogs my database, I guess, if I would store it in there, the password manager. - E01

In this quote, the participant describes the inconvenience of the password manager. Although he uses the manager, he weighs the inconvenience of the password manager against the significance of the account before deciding if he will use the manager for that account. Another participant described the same technique, but said that he made his decision on whether the website collected financial information. Yet another participant described a kind of thresholding process for determining which accounts got added to his password manager:

If this [password resets] happens more often than, I don't know, a bunch of times, then I will just use 1password to remember that password. - E09

A few participants said that after awhile, they memorized their frequently-used passwords. These participants had other systematic strategies for remembering passwords, but they explicitly remarked on the significance of frequently-used passwords.

For those accounts I use frequently, or not even that frequently, I know all by heart. - E04

If I use a password more often, then at some point I automatically remember the password. - E05

\section{Forget Your Password}

The expert users demonstrated awareness of specific threats in the interviews. When we asked about password changes, several experts referenced having changed their 
passwords in response to Heartbleed, a security bug in the OpenSSL library that necessitated widespread password changes ${ }^{1}$.

Well, there's, there's been a couple of incidents like, uhh, my laptop got stolen at one point, or... Or maybe you hear, like, a serious vulnerability like Heartbleed, and that's when you think that, that this might be a time to change passwords.- E07

In this quote, the expert participant describes two situations where he changed his passwords. One was a situation specific to him: his laptop was stolen and he was concerned that the thief might have gained access to his accounts. The second was a worldwide security bug that affected millions of users. Other participants mentioned similar scenarios:

Yeah, because, I just know the news about all these hacks and all these breaches. .. - E05

\section{Live with Your Password}

Even though passwords are presumably a subject of interest for people employed as security experts, our participants still described difficulty and frustration with password management. One participant described assigned random passwords as "ridiculous string[s] of horror" (E03). Another participant described how he avoided creating and managing passwords by keeping a minimal online presence. He accomplished this by relying on his spouse:

I try to shove off all my passwords to let my [partner] manage it. - E10

\subsection{Discussion}

Experts' password management strategies seem to fit into the same life cycle pattern that we found in non-experts' habits. While experts' passwords progress through the same stages, they incorporate some new strategies and techniques to proactively help

\footnotetext{
${ }^{1}$ The Heartbleed vulnerability was discovered in May 2014, after the initial interviews were completed, so it was not a particular vulnerability that could yet have affected the non-expert interviewees.
} 
them keep their accounts secure. Likewise, experts were applying similar rationing strategies to non-experts, devoting additional effort to important accounts and compromising for less crucial accounts. In this section, we compare the experts' behaviour to the non-experts from the earlier interviews, and provide some recommendations for non-experts based on the experts' practices. Finally, we identify areas where problems remain for all users.

\subsubsection{Comparing to Non-Experts}

In many ways, the behaviour described by the expert participants was very similar to that described by the non-experts in the earlier interviews. Experts do face the same problems as non-experts, and their coping strategies are remarkably similar. Many of the behaviours identified in the earlier interviews were reiterated by the expert participants. Experts have problems remembering assigned passwords, reuse their passwords, and forget their passwords. They write their passwords down, reuse the same password for long periods of time, modify those long-used passwords for further reuse, and guess at their own passwords. However, expert users were able to better protect themselves by pre-considering their security strategies, and by using their additional knowledge to better focus their efforts on areas of need.

One of the problems of password management is handling the unusual circumstances that arise in everyday life. Experts and non-experts face these same pressures - to show pictures to family, to share music at a party, to retrieve an address from an email, and others. Experts and non-experts both described these kinds of situations, and how they sometimes necessitated unusual password behaviour. Experts were less likely to change their behaviour in these scenarios, but even some of them acknowledged that social and cultural pressure could lead them to behave insecurely by entering their passwords on unknown computers.

One hallmark of the expert participants was their consistency, even in unusual situations. The non-experts made many ad hoc decisions. They described situations where they did not know what to do, and conflicting behaviour in different situations. They seemed to make decisions in the moment, rather than adhering to pre-set rules. Experts, however, seemed to have clear consistent rules for their behaviour. In the interview, we asked about the same scenario (creating a password) in different contexts. 
The expert participants seemed confused about the apparent question duplication, and always answered something like "same as before". Non-expert participants tended to describe different behaviour for creating passwords in different situations, and this inconsistency can lead to future confusion about what kind of password was created.

The expert participants in our interviews were well-informed about computer security threats and risks. This is unsurprising, but seemed to be a key part of their success. With this knowledge, they were able to focus their attention on the real risks and invest their efforts in effective strategies to protect the accounts they valued most. Their rationing technique was similar to what the non-experts described, but the experts were using distinct strategies for valuable accounts that allowed them to better protect these accounts. The experts were also careful not to waste effort on unimportant accounts.

Experts used the same coping strategies as non-experts for many of their accounts. They reused passwords, wrote passwords down, and created new passwords by making slight variations of older passwords. However, they combined these possibly insecure strategies with more careful habits for accounts where they were strongly concerned about security. One way they accomplished this was by using a password manager to generate and store passwords for high-value accounts, while reusing old passwords across other, lower-value accounts.

\subsubsection{What Do Experts Do Right?}

The purpose of the expert interviews was to better understand how experts are managing passwords, but also to see what can be learned from the practices of experts and adapted to help non-expert users manage their passwords.

Our interviews suggest that both experts and non-experts treat accounts with different requirements differently, but that the experts' consistency gives them an advantage in managing passwords. The experts in our study used password managers in combination with password reuse and other less secure coping strategies. They acknowledged the additional effort of using the password manager, but had selected the accounts where this effort was worthwhile. By using the password manager only on those accounts, they were effectively budgeting their time and effort to protect their most valuable accounts. 
We suggest that end users should be able to apply these consistent strategies to strongly protect the accounts they care about most, while not wasting effort on other accounts. The process of setting up a password manager can be daunting to end users, but by selecting a small set of accounts for initial set-up, the task is made significantly smaller. For example, users could select three important accounts, install a password manager, and add those accounts to the manager. Instead of attempting to solve their whole password problem, users should focus on the accounts that matter most to them. This incremental approach is scaleable, and it is possible that once the password manager is set up and in use, the user may want to use it for other accounts.

The experts in our interviews were largely taking advantage of available strategies. They were mostly working with existing tools (open source and commercially available password managers) that are easily available online. We cannot expect that every user will be able to create a robust password generation algorithm, but most of the expert tools are available for use by anyone. This means that the expert approach really is accessible to non-experts.

\subsubsection{Remaining Problems with Password Management}

Though the habits of experts can help address some of the issues with passwords, other problems still remain. Here, we highlight a few issues that were identified as problems during the interviews.

Password changes were a source of tension for most users. Experts were more likely to say that they changed their passwords than non-experts, but most experts said they changed their passwords only rarely and commented on the difficulty of the process. Participants commented on how the password change process can look different for every website, and finding the correct page and going through the password change process can be time-consuming. One participant commented that he wished there was a way of systematically changing passwords for all accounts.

Password changes are also a usability issue of password managers. To take advantage of the random password generation functions in most managers, passwords for existing accounts must be changed. This process can discourage all kinds of users 
from adopting password managers. The ability for password managers to communicate directly with remote sites via an open, auditable password management API could reduce friction in the password change process, and even allow automated bulk password changes. Such an API could also be used to notify the password manager of password expiration or known password compromises.

A lurking theme in our interviews was the usability of password managers. Although none of the experts in our study complained about the usability of their password managers, their unwillingness to make them their default password management strategy seems to point to some kind of issue with their usability or usefulness. Since their usefulness is evident, the issue is likely usability. Since there were no specific complaints about particular managers, the issue might simply be that password managers require an additional effort and a few extra clicks when logging into websites. Once again, a better ability for password managers to communicate with websites could address these issues by streamlining password management tasks.

\subsection{Conclusion}

Password management can be a struggle for everyone, even experts in computer security. Our interviews with experts about their password management habits showed that they use a combination of password management strategies to carefully allot appropriate security to individual accounts. Several experts relied on password reuse and other less secure coping strategies for lower-value accounts, but used a password manager to generate and remember random passwords for high-security accounts.

The expert approach suggests that all users could improve their password management strategies by using a password manager for their most valued accounts. Explicitly identifying a small number of high-priority accounts is a natural extension of end users' existing rationing strategies, and the comparatively small effort to better protect those accounts could significantly improve users' security. Additionally, this incremental approach could scale to protect more than just the most valuable accounts and could foster better password habits for all accounts.

Of course, expert knowledge does not solve all usability issues with passwords. Problem areas for password management include the usability of password managers and 
the ease of password changes. Although the expert approach cannot remedy all password management problems, it can suggest practical advice and strategies to help end users manage passwords in their daily life. 


\section{Chapter 7}

\section{Surveying Users about Password Management}

\subsection{Introduction}

As we emphasized in Chapter 5, the work on the password life cycle was theorybuilding, not theory-validating. Following the development of the life cycle theory, we grew interested in how the model would hold up in a broader population.

Our Grounded Theory analysis showed that individual users use passwords in a cyclical method, first creating passwords, then committing those passwords to memory until they are compromised, forgotten, or out of date, at which point a new password is then selected, reused, or adapted from another password. Users ration their effort into maintaining and managing passwords, devoting additional effort to important passwords and accounts, and saving attention from accounts that need it less.

The theory described in Chapter 5 was developed from a sample of users from the Carleton University community that included both students and members of the administrative staff. We reached saturation and stopped interviewing after 27 participants when we realized we were hearing little new in each interview. Although our model reflects the content of our interviews, an open question remains about whether the theory is applicable to larger populations. 
To investigate how the password life cycle model generalizes in a more diverse population, we conducted a survey-based study. The survey asked a wide variety of questions about exactly what users do to cope with the demands of passwords, and how well users feel they are handling those demands. By better understanding how the behaviours and themes that we identified in the interviews occur in a larger population, we can better design ways to either safely support these behaviours, or to discourage and replace them with safer behaviours.

\section{$7.2 \quad$ Study}

We designed a survey instrument based on the findings of the life cycle. We decided to use a survey for the research because it did not require the presence of an interviewer, and could thus be easily and efficiently distributed to a large group of respondents.

We developed our survey based on the phases of the password life cycle. The survey had seven question groups, each focusing on a different aspect of password management. These groups included sections on choosing passwords, reusing passwords, saving passwords, changing passwords, and perceptions of password security. We also included a group with some basic questions about password and account use, as well as a demographics section. The questionnaire was approved by the Carleton University Research Ethics Board, and the full questionnaire can be seen in Appendix C.

The section on password use echoed the questions asked in the life cycle interviews, and was included to give a comprehensive picture of respondents' online habits. It also provided a means of comparing the online sample to the two in-person samples, non-experts and experts. The demographics questions were also similar to those included in the interviews, and were again included to give a better understanding of the sample.

The main question groups were each based on a theme identified in the axial coding procedure during the Grounded Theory analysis of the original data, though they were not identified as such to the respondents. In developing each group, we carefully combed the results of the qualitative analysis and developed questions based on the behaviour revealed in the interviews. For example, in the section on choosing passwords, we asked respondents to rate how frequently they included different types of personal information, including birthdays, names, and addresses, in their passwords. 
The survey used a variety of question types, including Likert scales, multiple choice questions (i.e., "choose all that apply..."), yes/no questions, numeric fill-ins, and a few text fill-ins. For many questions, we asked respondents to rate how frequently they engaged in a specific behaviour rather than asking a yes/no question. This

was intended to capture the full spectrum of behaviour, and some of the diffidence revealed in the interviews. In the interviews, respondents frequently told us that they did something occasionally, and were unwilling to strongly commit to a statement of the format "I do ...".

In line with best practice for survey design, we designed our survey to minimize the effects of response bias. For questions with multiple parts, we randomized the order of presentation for subquestions. This was done to prevent an effect from respondents who focused more on the early questions. We also reversed the wording on some questions so that respondents would not be tempted to simply fill in the same response all the way down a question. Finally, we included a few duplicate questions that asked the same questions in different parts of the survey, and used these to evaluate whether respondents were reading the survey carefully.

We conducted the survey on Amazon's Mechanical Turk [4], an online micro-working platform that allows access to participants from around the world. We chose Mechanical Turk to give us easy access to a large and more diverse survey sample. Our survey was implemented using LimeSurvey [113], an open source survey platform that is run from our own servers. This meant that all data was collected and stored only on our secure servers.

\subsubsection{Pilot Testing}

We conducted two pilot tests of the survey. The first pilot had 10 respondents, and the second had 100 respondents. The pilot testing gave us a better understanding of how real respondents would answer our survey questions, and whether we were collecting the right kind of data. The testing also allowed us to ensure that our survey software and setup were functioning correctly.

We conducted preliminary analysis on the pilot study data to help us understand the collected data, and the trends in responses. Following this analysis, we made 
minor changes to the survey questionnaire. We removed duplicated questions, and turned a previously long-answer question into a multiple choice question because most respondents ignored it in the pilot test. We improved the answer options on a few questions, and clarified some wordings. We also broke one long question into three shorter questions to help respondents respond more easily.

\subsubsection{Survey Participants}

We collected 348 complete responses to the actual survey (three questionnaires from duplicate respondents were removed).

$60 \%$ of the respondents were male, and $38 \%$ were female (the remaining $2 \%$ did not state their gender). The survey sample was quite young - $90 \%$ of participants reported being 45 or younger. 90\% of participants' IP addresses were in the United States, $7 \%$ were in India, and the remaining participants were from countries scattered across the globe. The majority of participants (95\%) reported English as their mother tongue, and the next most common first language was Tamil (2\%). Respondents' educational achievement was high: $45 \%$ reported having a university degree (undergraduate or graduate) and $23 \%$ had a college or professional qualification. Respondents reported a diverse list of professions, and only $4 \%$ reported that they were students.

\section{$7.3 \quad$ Results}

We present the survey results organized by question group.

\subsubsection{Password Use}

The first question group asked respondents about how many accounts and passwords they had. The questions in this section were very similar to those asked in the interview, and were intended to provide a basis for comparison. Table 7.1 shows descriptive statistics for the number of reported accounts, accounts used every week, 
Table 7.1: Password use statistics for all respondents.

\begin{tabular}{|l|r|r|r|r|r|}
\hline & Mean & SD & Median & Maximum & Minimum \\
\hline Number of accounts & 25.2 & 15.5 & 21.0 & 97.0 & 3.0 \\
Accounts used in a week & 16.2 & 11.0 & 13.0 & 85.0 & 1.0 \\
Unique Passwords & 8.5 & 8.4 & 5.0 & 60.0 & 1.0 \\
Reused Passwords & 3.2 & 2.0 & 3.0 & 16.0 & 1.0 \\
\hline
\end{tabular}

unique passwords, and reused passwords. The median number of reported accounts was 21, and respondents said they use 13 of these accounts in an average week. Respondents reported having a median of 5 unique passwords, and 3 reused passwords. These statistics are similar to what was reported in the earlier interviews (Section 5.3).

\subsubsection{Choosing Passwords}

Almost every account creation involves choosing a password, so users must have reliable coping strategies for this scenario. In this question group, we asked respondents about the kinds of information they include in passwords, other considerations that may affect password choices, and the kinds of habits that users maintain for adjusting passwords to meet password policies.

Figures 7.1 and 7.2 show the distribution of frequencies with which respondents reported using personal information in passwords. The most common response for all items was that respondents "never" included that type of information, but $41 \%$ of respondents indicated that they often or always include at least one of these types of personal information in their passwords.

We asked respondents how they change their passwords to comply with password policy expectations. Almost half of all respondents said that they "often" or "always" reach for a particular digit (47\%), capitalize a particular letter (46\%), and $38 \%$ use a particular symbol in this situation.

We also asked respondents about whether their passwords corresponded to external factors such as time periods, usernames, and password policies. Almost half of respondents said that their passwords corresponded to a particular password policy (49\%), but fewer reported that they matched passwords with time periods $(23 \%)$ or usernames $(16 \%)$. 


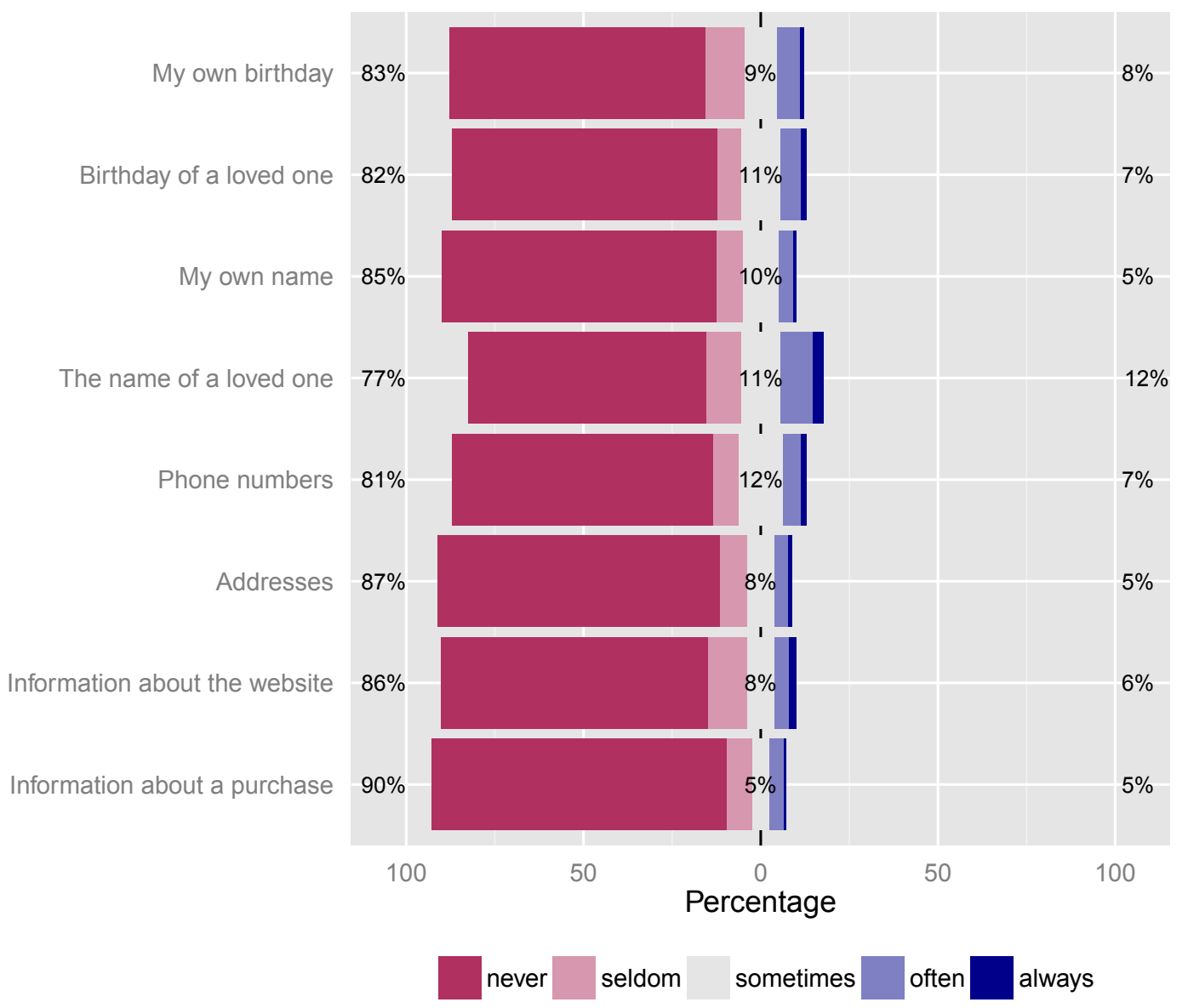

Figure 7.1: Responses to rating scale questions about how frequently respondents include various types of personal information in their passwords. 


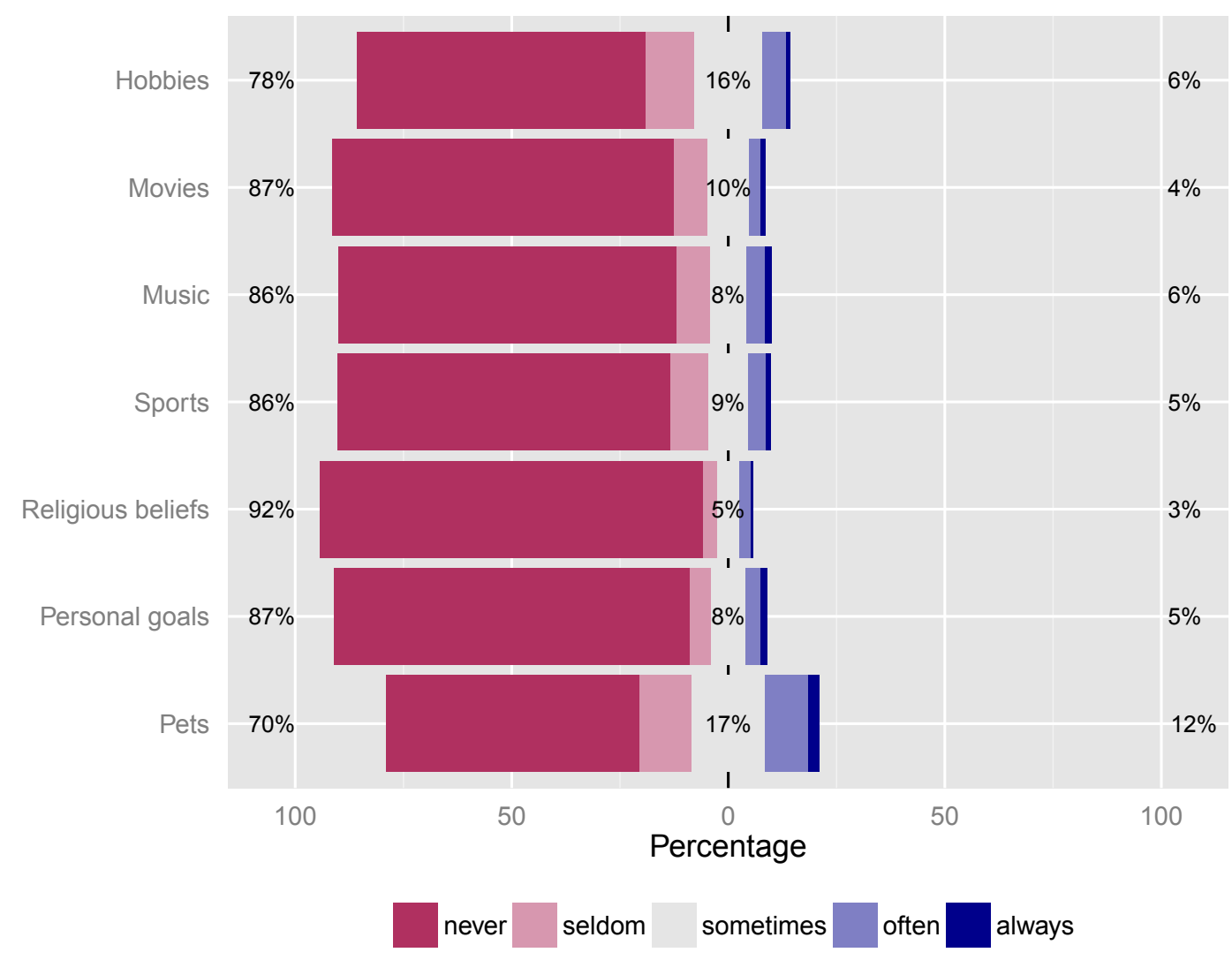

Figure 7.2: Responses to rating scale questions about how frequently respondents include various types of personal information in their passwords. 


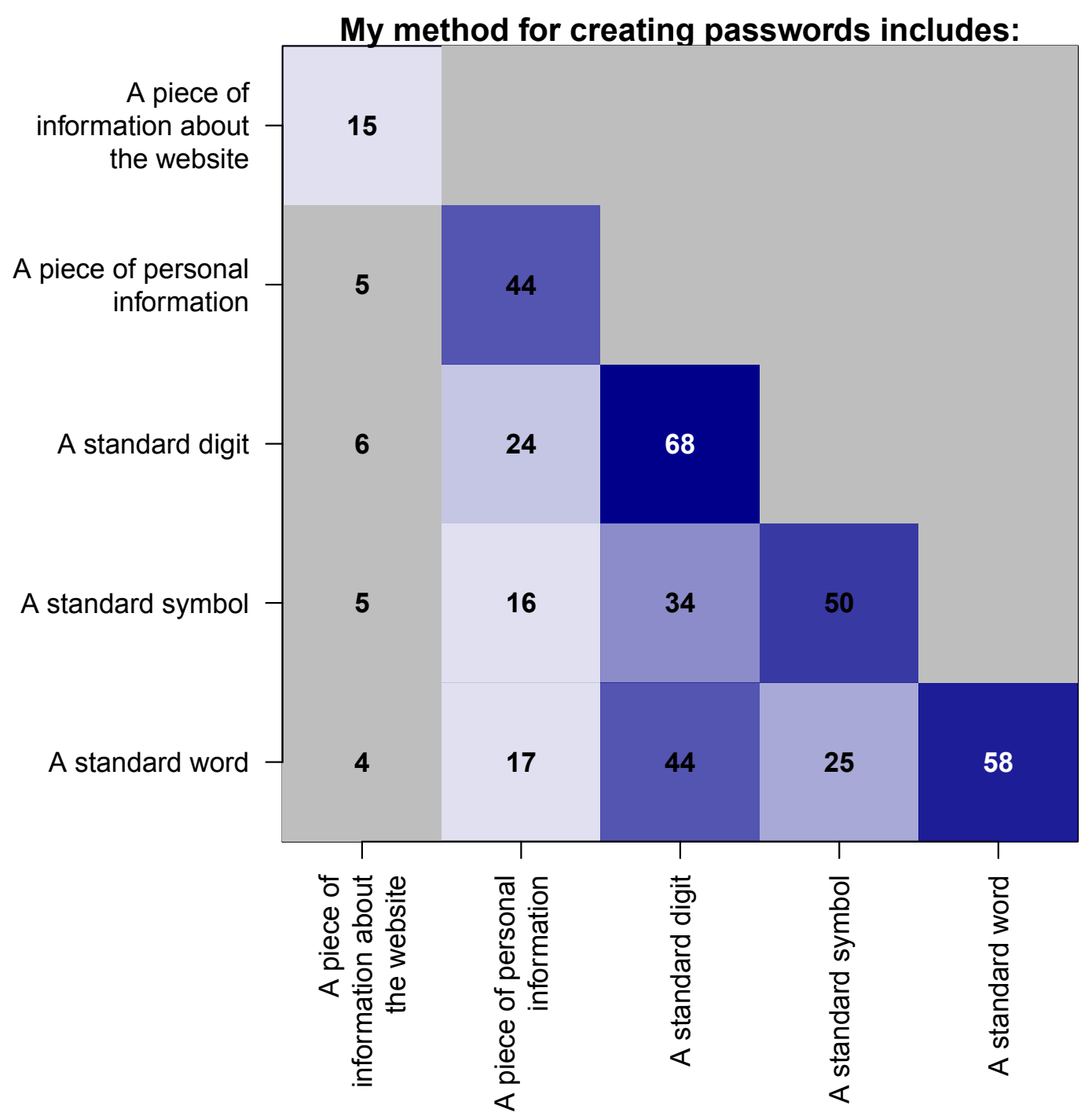

Figure 7.3: Density grid showing the frequency of pairs of co-incident elements that respondents include in their passwords. Darker colours indicate higher frequency, and the diagonal boxes show the total frequency of selections for that element. For example, a standard digit is the most common element that respondents included in passwords, and a standard word and digit was the most common combination of elements. 


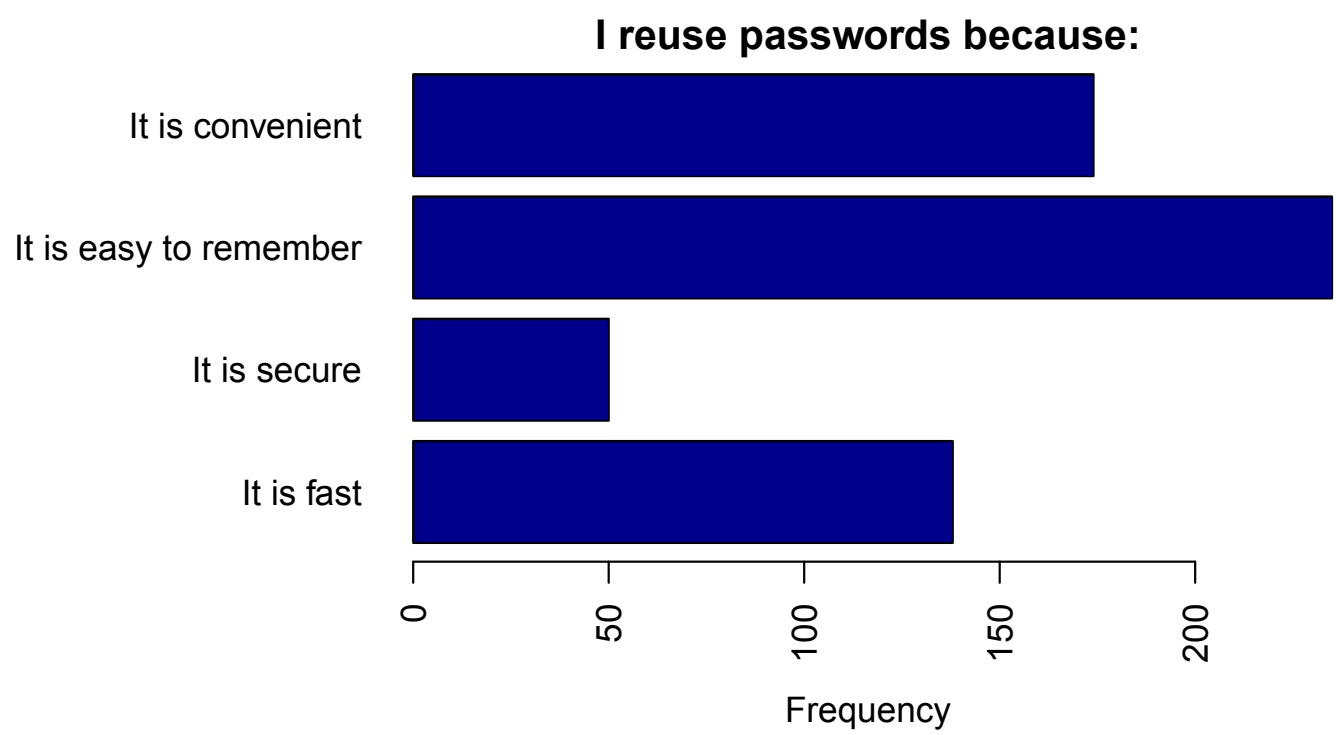

Figure 7.4: The reasons that respondents reuse passwords. Note that respondents were allowed to choose more than one option, so the bars do not sum to the number of respondents.

More than a third of respondents told us that they had a systematic way of combining information to create passwords (39\%). Of these respondents, the most commonly used elements were a standard word and a standard digit (Figure 7.3).

\subsubsection{Password Reuse}

The reuse question group focused on the strategies that users employ to manage password reuse. The majority (75\%) of respondents in our survey reported reusing passwords, and the majority of those (99\%) said they reused passwords because reused passwords are easier to remember (Figure 7.4).

With such a high rate of password reuse, it is unsurprising that respondents reported reusing passwords on many similar kinds of accounts. Figure 7.5 shows the distribution of frequency for many different kinds of reuse. In all situations, most respondents reported reusing passwords "often" or "always". We also asked respondents who reported that they reuse passwords about the accounts on which they sometimes avoid reusing passwords. The most common accounts where participants avoided password reuse were online banking websites (62\%) and websites that store credit card information (43\%). 


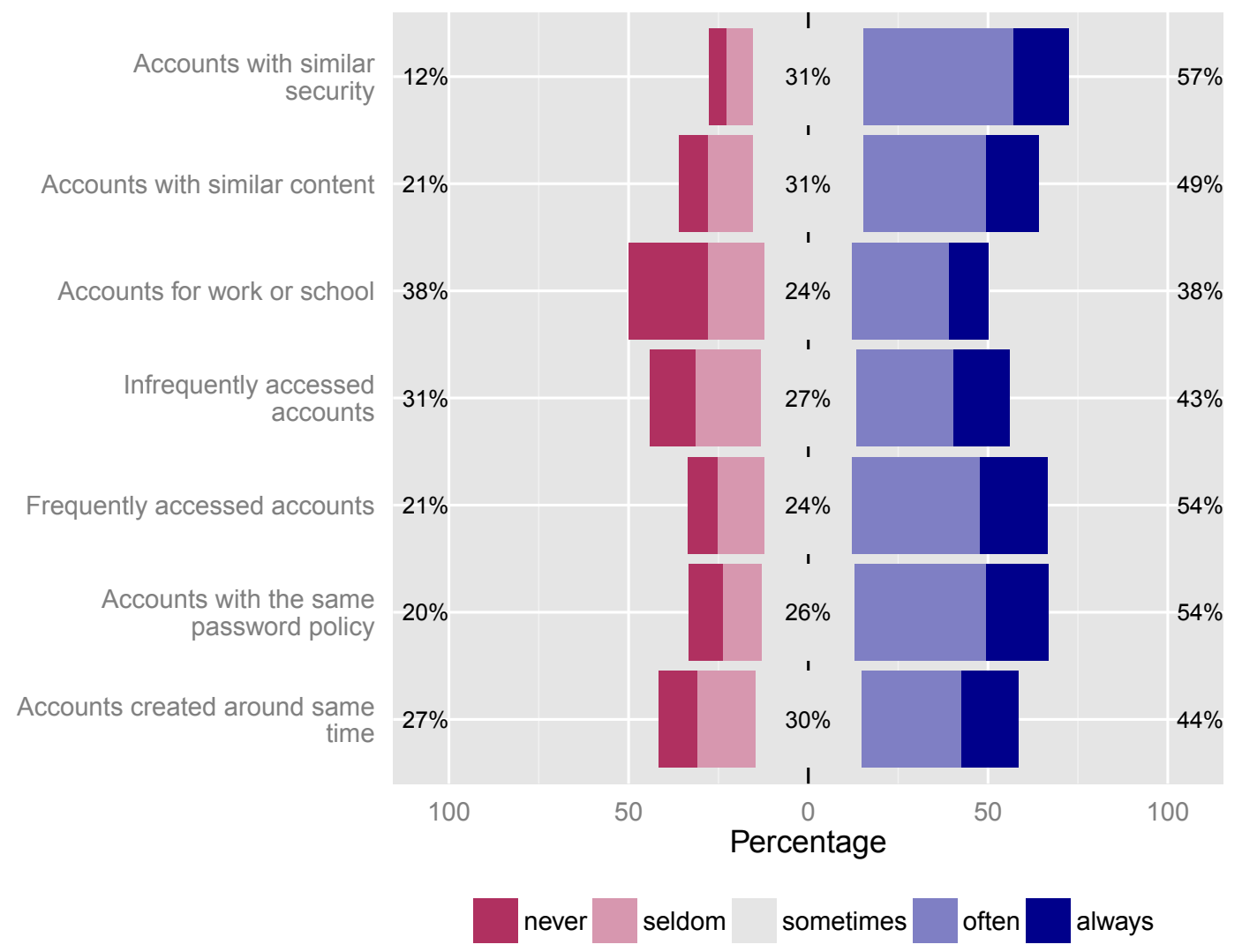

Figure 7.5: Responses to rating scale questions asking about how respondents reuse passwords. 


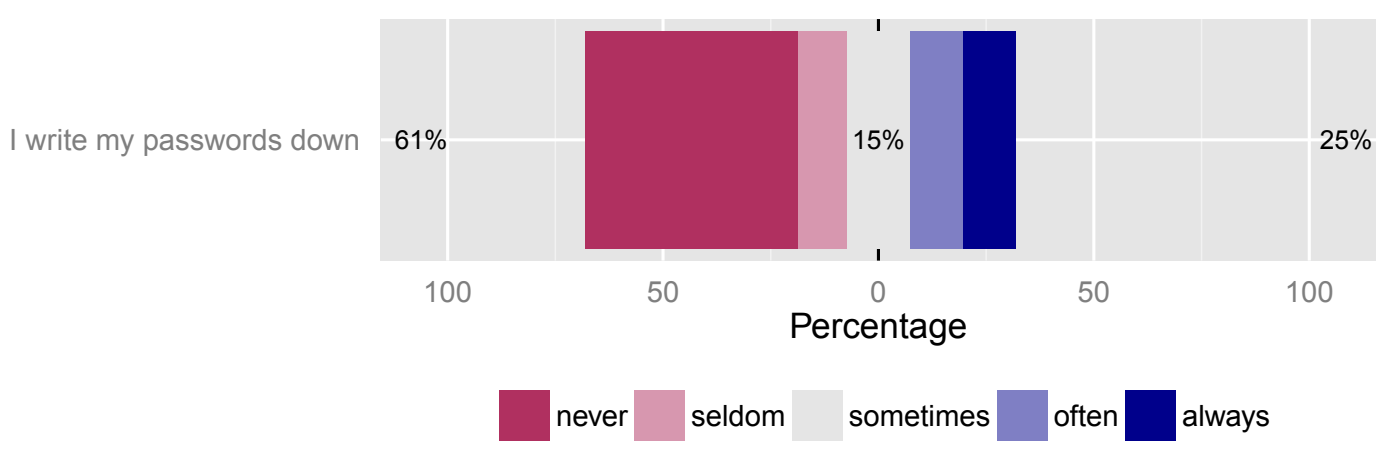

Figure 7.6: Frequency with which respondents reported writing passwords down.

In the interviews, many respondents referred to a particular password when discussing reuse. About two-thirds (60\%) of respondents said they had a "go-to" or primary password. Of those, most said that they created variations of this password to use on different accounts (81\%), and that they had a standard way of creating these variations $(71 \%)$.

\subsubsection{Saving Passwords}

Users who have not memorized all of their passwords must use some means of saving those passwords. In this section of the survey, we asked respondents about their use of password managers (browser-based and dedicated), as well as their password recording strategies.

Figure 7.6 shows the distribution of how frequently respondents reported writing their passwords down. The majority of respondents (61\%) reported that they "never" or "seldom" wrote their passwords down. Respondents were most likely to say that they recorded infrequently-used and high-security passwords (Figure 7.7). Half of the respondents (51\%) reported that they wrote their passwords down in case they needed them, and $43 \%$ said that they did not usually refer to their recorded passwords.

Only $17 \%$ of respondents reported saving their passwords in a dedicated password manager, but 50\% reported saving their passwords in a web browser. Of the respondents who reported saving passwords in the browser, respondents were most likely to say they did not save banking passwords, or passwords for accounts that saved credit card information (Figure 7.8). 


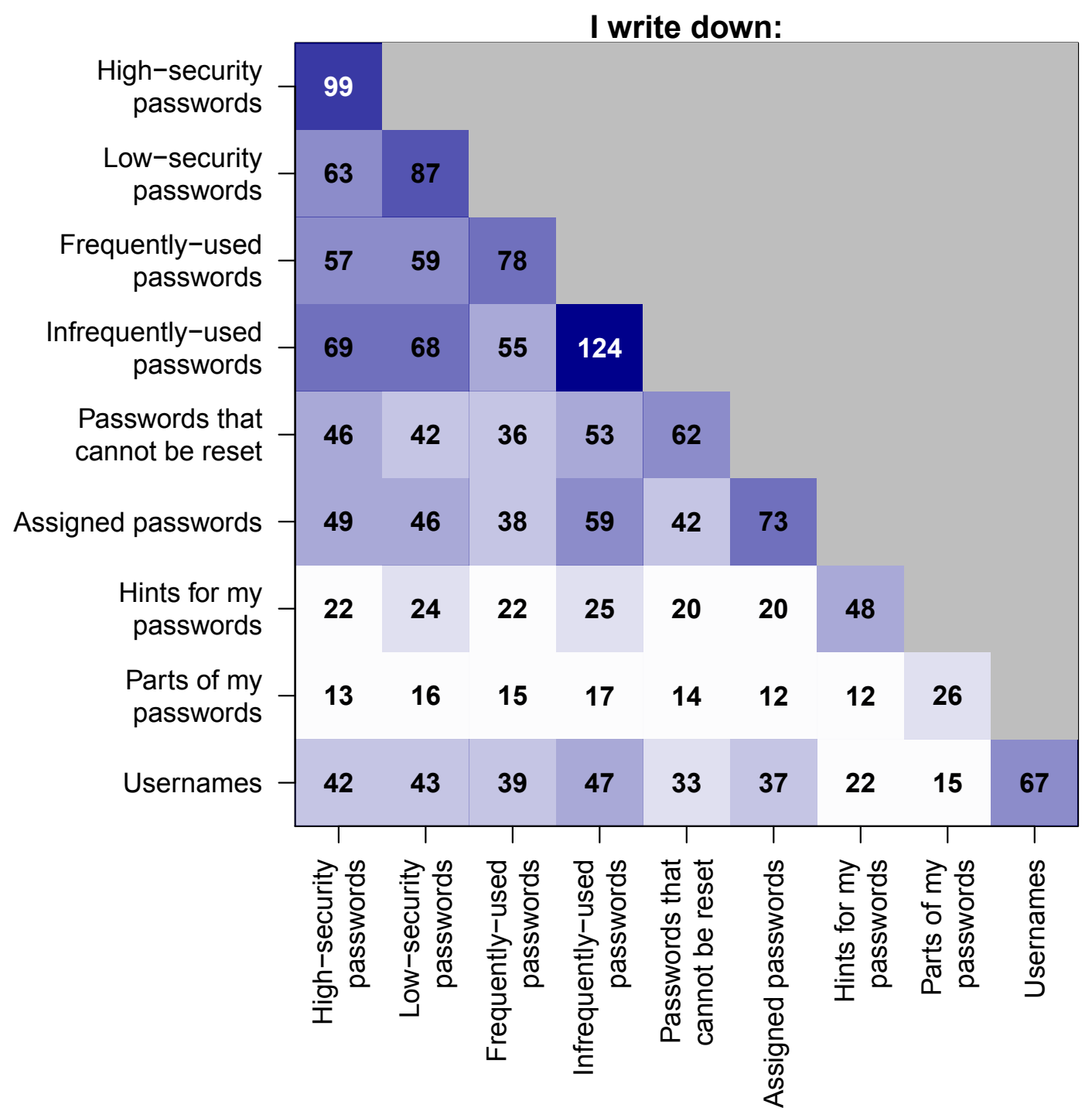

Figure 7.7: Density grid showing the frequency of pairs of co-incident types of passwords that respondents write down. Darker colours indicate higher frequency, and the diagonal boxes show the total frequency of selections for that element. For example, respondents most often write down infrequently-used passwords, and the most frequently written down combination is infrequently-used and high-security passwords. 


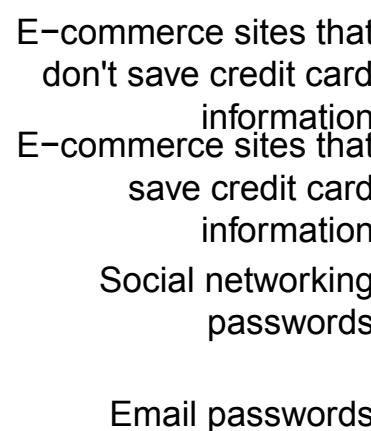

\section{I do not save these passwords in a web browser:}
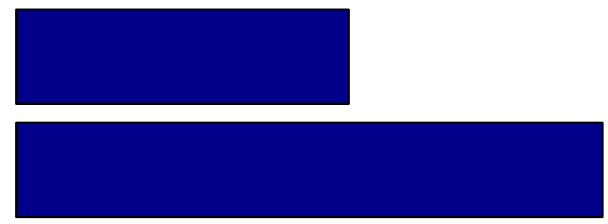

Banking passwords
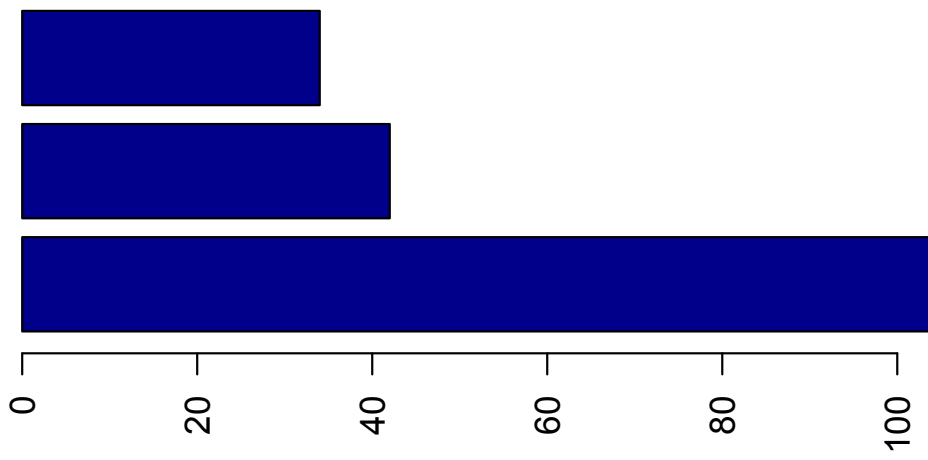

Frequency

Figure 7.8: Types of passwords that users do not save in the web browser. Note that respondents were allowed to choose more than one option, so rows do not sum to the number of respondents.

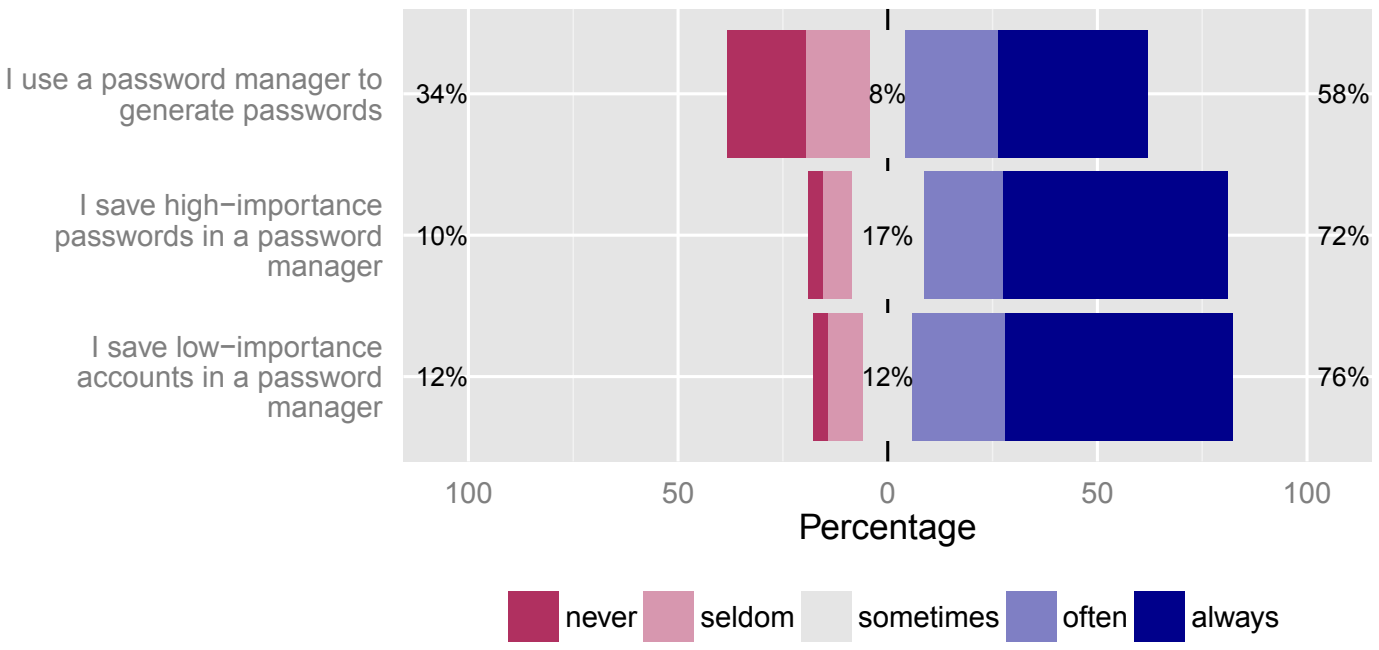

Figure 7.9: Responses to rating scale questions about how frequently users use password managers. 
Of the respondents who reported using a dedicated password manager, most reported using it for some combination of convenience and security. Most users took advantage of all password manager features: users reported saving both high- (72\%) and lowsecurity $(76 \%)$ passwords in the manager, and slightly fewer $(58 \%)$ reported using the manager to generate passwords (Figure 7.9). Of the respondents who said they did not use a password manager, the most common stated reasons were a lack of trust (44\%), no need for one (36\%), and an unwillingness to set one up $(28 \%)^{1}$.

\subsubsection{Changing Passwords}

This question group asked respondents about password changes. It focused on three separate situations: a situation where respondents decided to change their passwords of their own accord, a situation where respondents were obliged by the account to change their password, and a situation where respondents changed the password because they had forgotten the previous password.

Only about one third of respondents reported changing their passwords on a regular basis, but approximately two thirds of respondents reported having changed their passwords in response to a specific event. In this case, the most common events were the compromise of an account and media coverage of a security breach (Figure 7.10).

Figure 7.11 shows the distributions of behaviours that respondents use to cope with forced password changes. The graph shows that respondents are most likely to choose an entirely new password in this situation (48\% agreement).

The majority of respondents said that they forgot passwords at most once per month, and Figure 7.12 shows the distributions of behaviours that respondents use to cope with forgotten passwords. Respondents were most likely to rely on the "forgot password" button ( $67 \%$ agreement), followed by using the verification questions ( $44 \%$ agreement) and guessing at the password (41\%). When respondents had to reset a forgotten password (Figure 7.13), respondents were most likely to agree that they would change the password to what they thought it should have been ( $42 \%$ agreement).

\footnotetext{
${ }^{1}$ Respondents were allowed to pick more than one choice, so percentages do not sum to 100.
} 


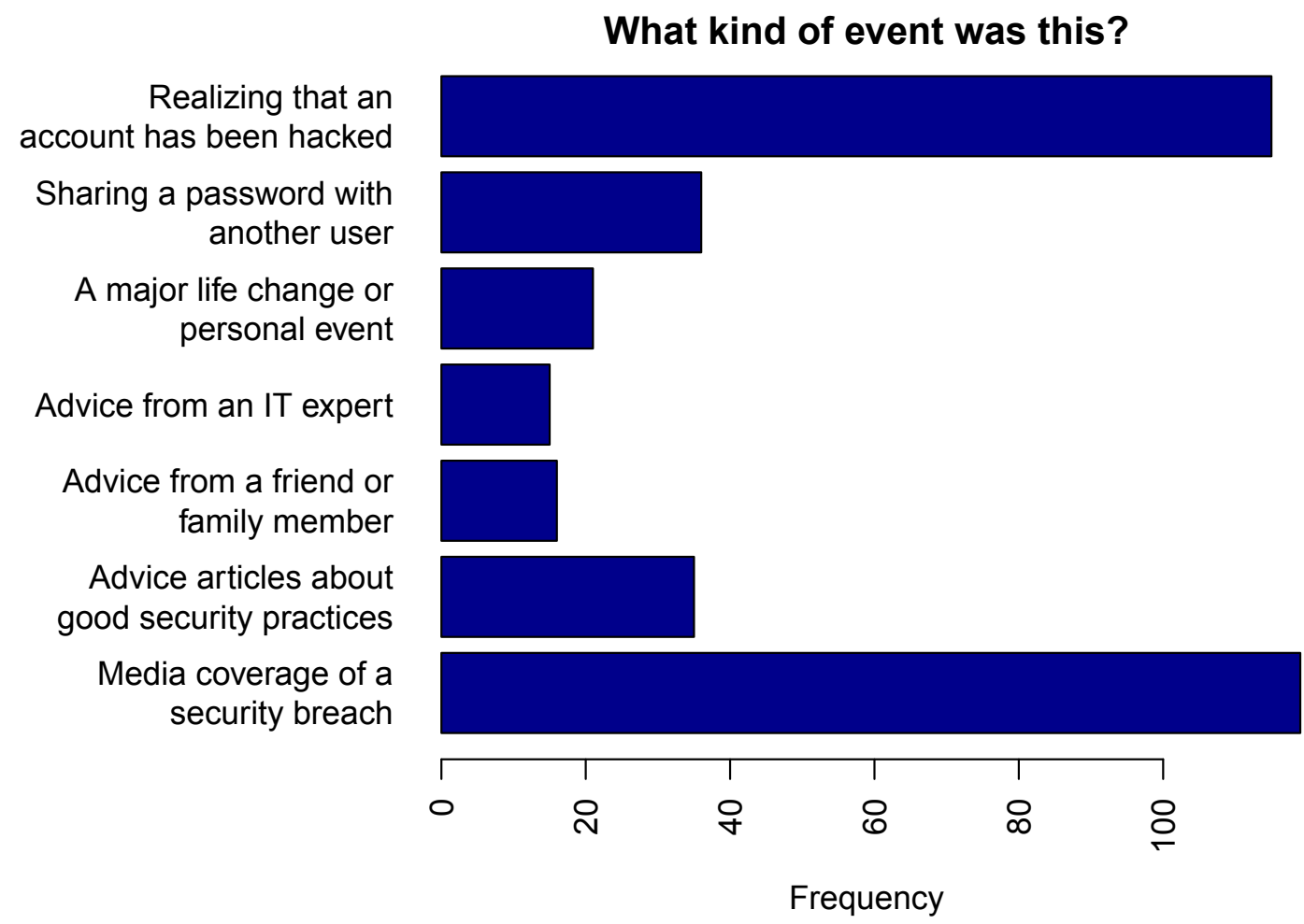

Figure 7.10: Types of events that motivate users to change their passwords. Note that respondents were allowed to choose more than one option, so the bars do not sum to the number of respondents.

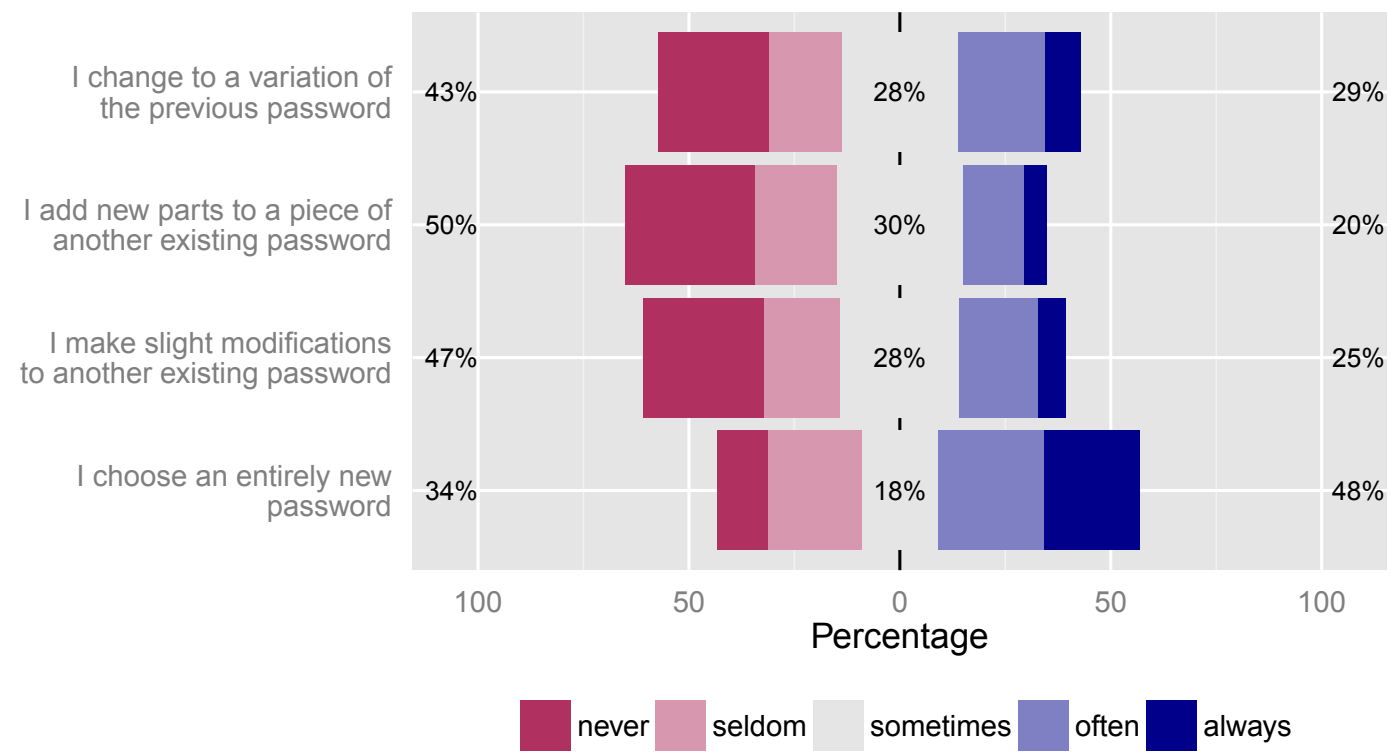

Figure 7.11: Rating scales showing users' strategies for forced password changes. 


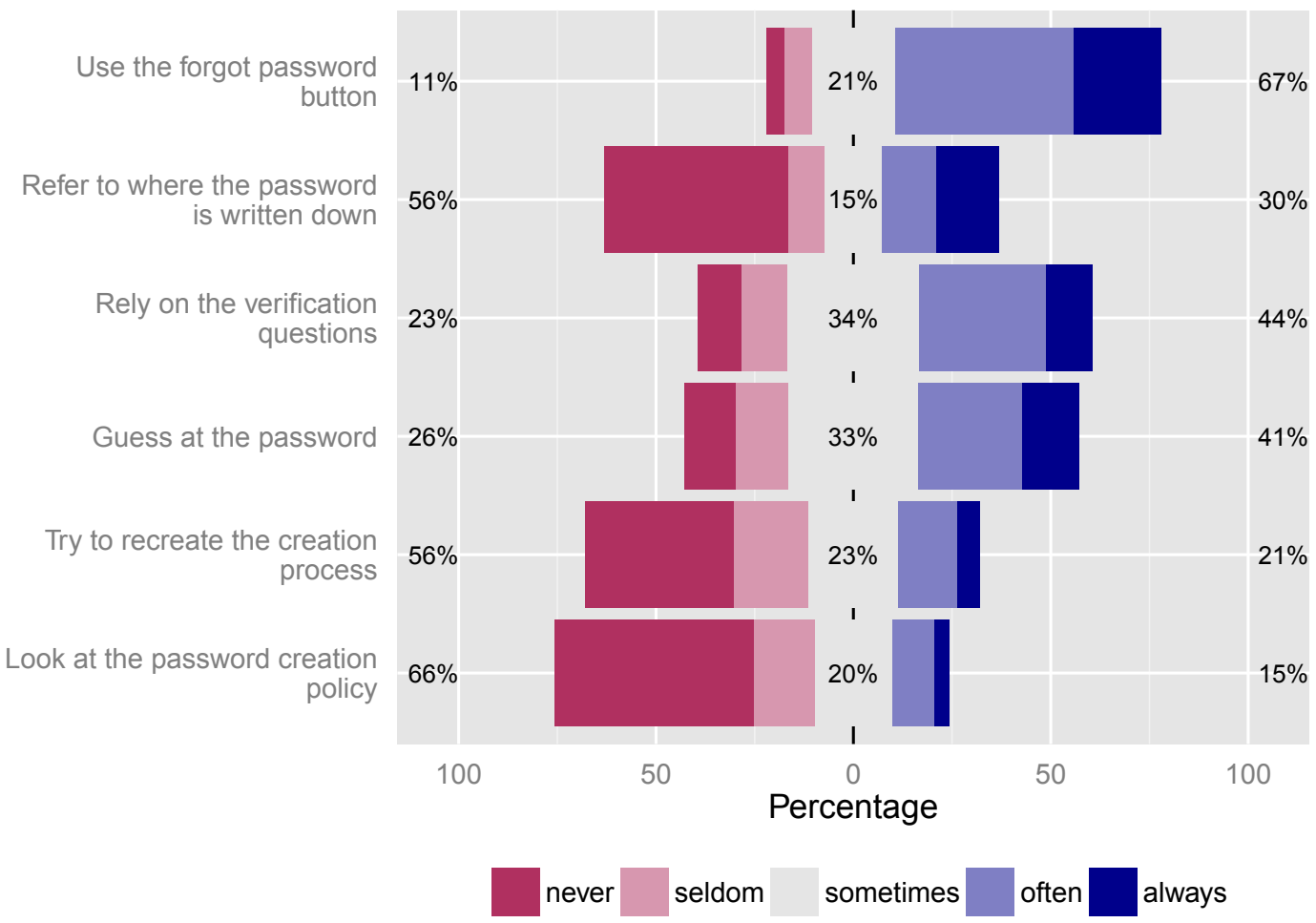

Figure 7.12: Rating scales showing users' coping strategies for handling forgotten passwords. 


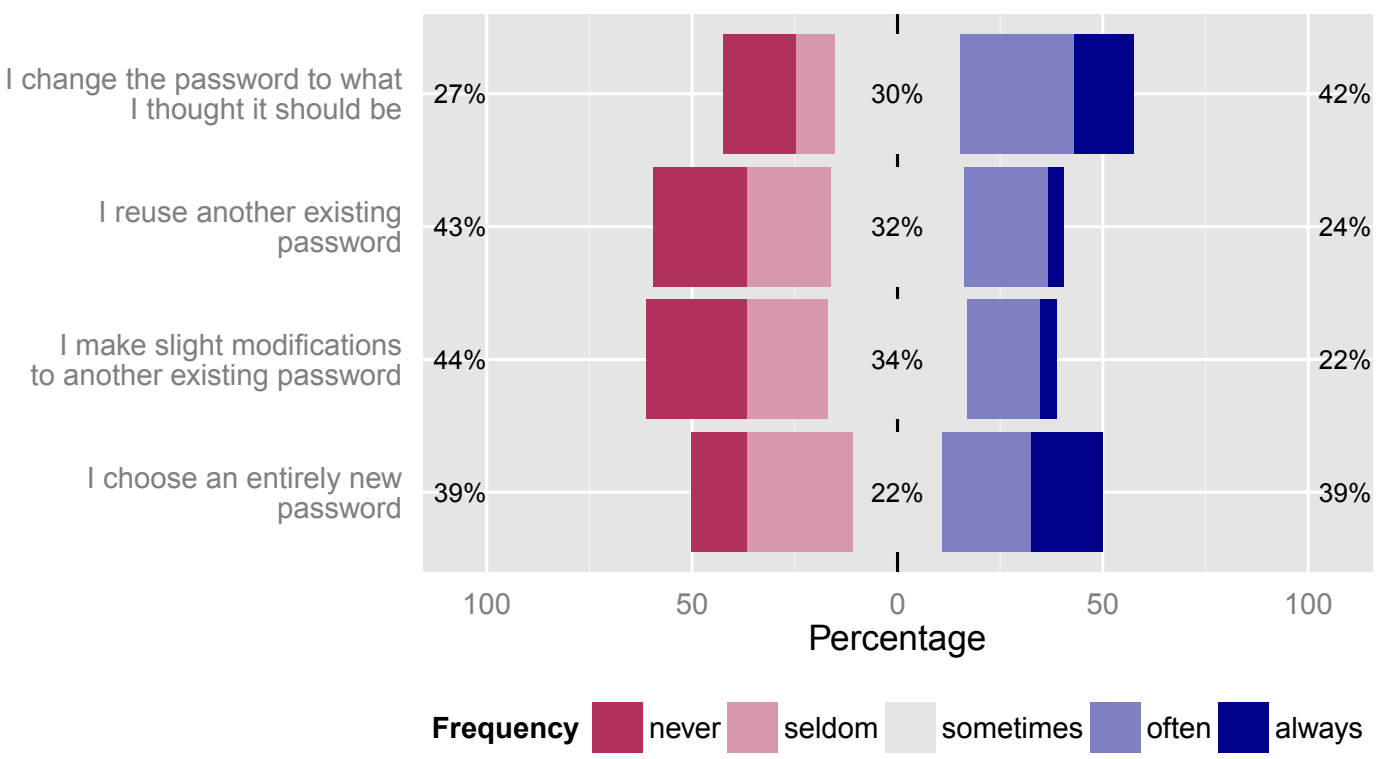

Figure 7.13: Rating scales showing users' behaviour when resetting forgotten password changes.

\subsubsection{Security Perception}

The final question group asked respondents to rate their agreement with a number of statements about security and password management. The purpose of these questions was to investigate how respondents perceive the amount of effort devoted to password management and the success of these efforts.

Figure 7.14 shows responses to a series of Likert scale questions about respondents' perceptions of their security behaviour. Respondents were generally quite positive about password management: $55 \%$ of respondents agreed that picking new passwords was easy for them, and only $35 \%$ of respondents agreed that it is difficult to keep track of passwords.

Most respondents (65\%) indicated that they believed they were adequately protecting their accounts, and felt that their accounts were unlikely to be attacked (reversed question, $61 \%$ agreement). However, the majority of respondents (78\%) agreed that they could do more to protect their accounts. Surprisingly, most respondents $(72 \%)$ disagreed with the statement "I do not have time to pay attention to security". 


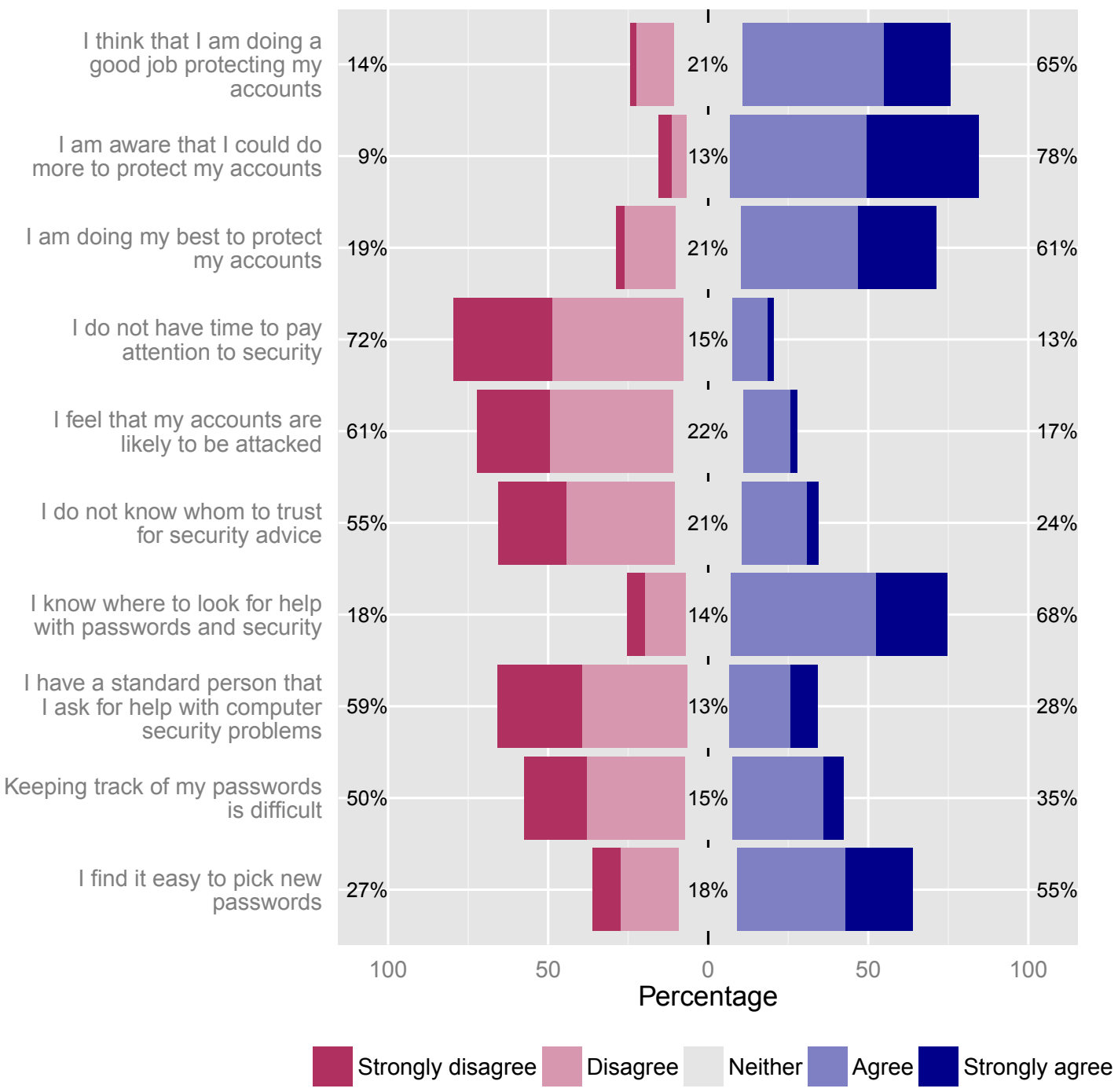

Figure 7.14: Rating scales showing perceptions of difficulty of security tasks. 


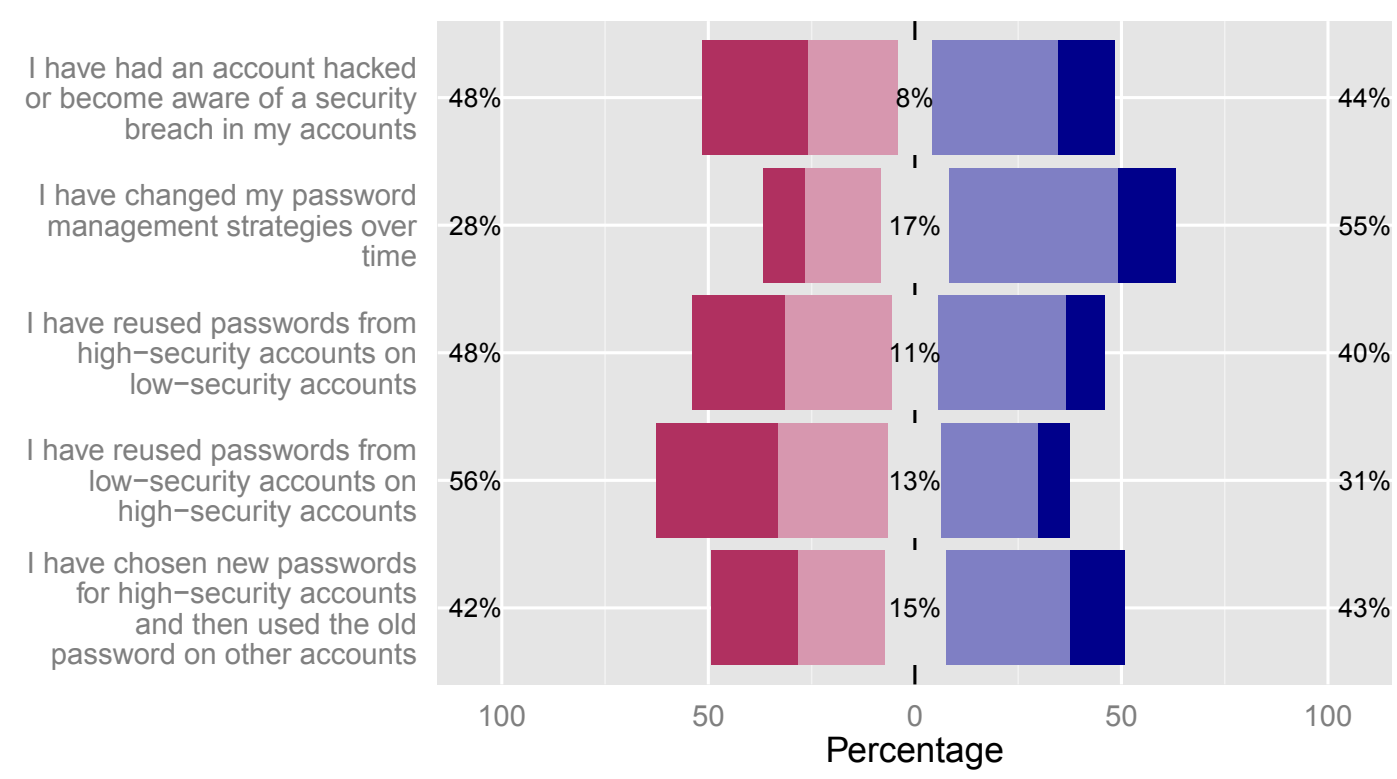

Strongly disagree Disagree Neither Agree Strongly agree

Figure 7.15: Rating scales showing perceptions of behaviour across time.

$68 \%$ of respondents agreed that they knew where to look for security advice, and whom to trust for security advice (reversed question, 55\% agreement). However, this source of trust was not a standard trusted person (59\% disagreement).

Figure 7.15 shows responses to questions about behaviour over time. 55\% of respondents agreed that they had changed their password management strategies over time. Respondents were split on their perceptions of how they had reused passwords: $40 \%$ indicated that they had reused passwords from high-security accounts on low-security accounts, and $31 \%$ indicated that they had done the opposite. Both of these kinds of reuse can be a major threat to the security of high-importance accounts, because users may be less careful about the security of low-importance accounts. $43 \%$ said that they had shifted passwords from one account to another by choosing a new password for a high-security account and then reusing the old password on other accounts.

Figure 7.16 shows responses to Likert scale questions about "security attention". We chose not to define this term in the questions (although we did put it in quotes for special consideration), and left respondents to interpret it as their personal accumulation of time, attention, knowledge, education, etc. 


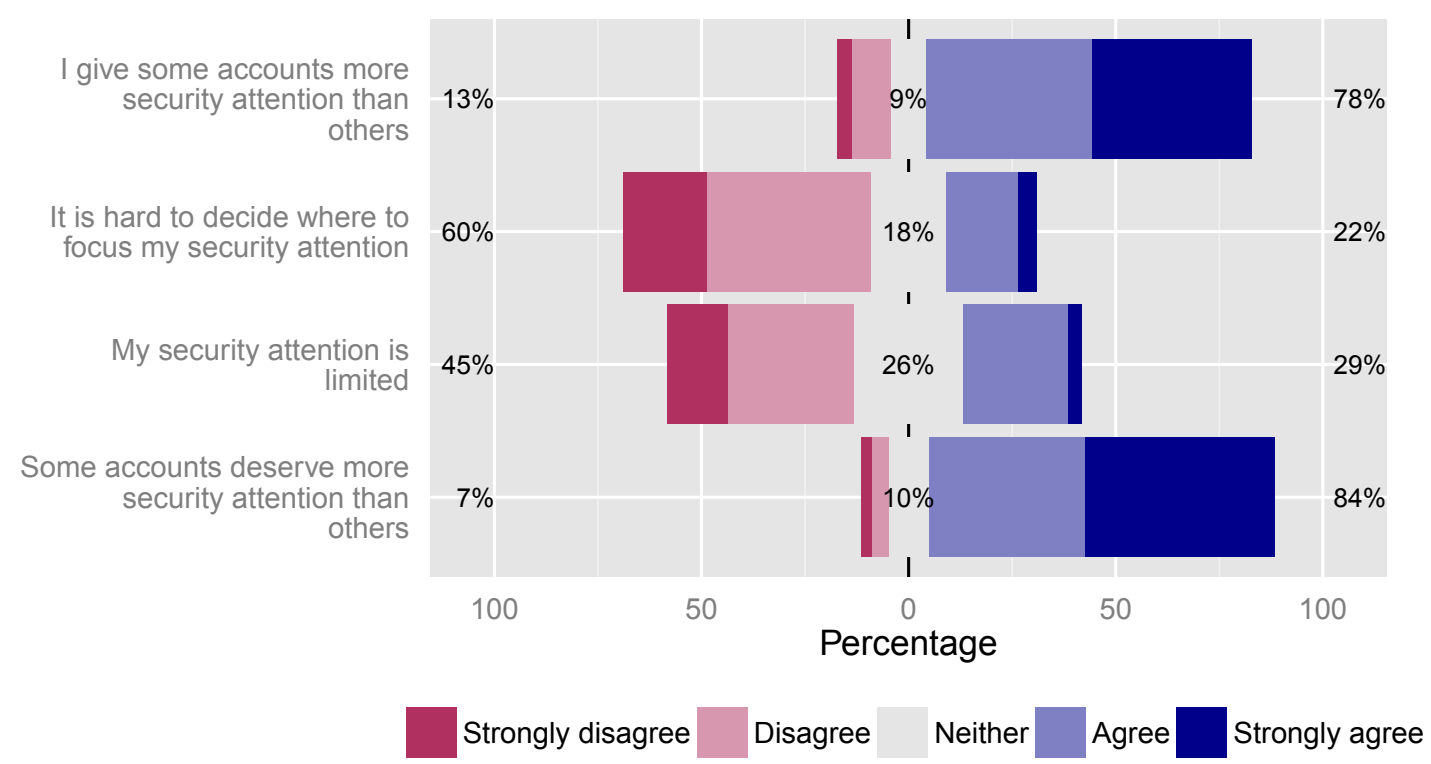

Figure 7.16: Rating scales showing perceptions about attention paid to security.

A large majority of respondents agreed that they give more security attention to certain accounts (78\% agreement), and that some accounts deserve more security attention than others ( $84 \%$ agreement). Respondents did not seem to strongly agree that budgeting security attention was a problem: only $29 \%$ of respondents agreed that their security attention was limited, and only $22 \%$ agreed that they had trouble deciding where to focus their security attention.

\subsubsection{Demographic Trends}

Following our evaluation of the entire data set, we examined differences between demographic groups. We broke our data set into groups by gender, age, computer expertise, and security expertise. We found no major differences between men and women, or differences based on respondents' self-reported computer expertise. In the following section we highlight notable differences between the groups distinguished by age and security expertise.

This analysis was conducted post-hoc, and not based on a priori hypotheses. The purpose of this survey was not to look for statistical differences between groups, but 
rather to see how aspects of the model applied to a broader user group. For this reason, we did not conduct any statistical tests. In future work, it would be interesting to deliberately sample different demographic groups and to investigate the differences.

\subsubsection{Age}

We divided our sample by age into younger users (younger than 35) and older users (35 and older), and found a few noteworthy differences in reported behaviours. Our sample was skewed toward younger users: there were 247 younger users and 98 older users $^{2}$.

Older participants were more likely to write their passwords down: $46 \%$ of older users reported writing their passwords down, as opposed to $17 \%$ of younger users.

Older users appeared to be moderately more careful about reuse, particularly in sensitive situations. Younger users were more likely to say that they reused passwords often or always on all kinds of accounts (Figure 7.17), and especially more likely for accounts concerning work or school. $53 \%$ of older users said that they seldom or never reused these passwords, compared with only $32 \%$ of younger users.

\subsubsection{Security Expertise}

We asked participants to self-report their computer security expertise. About one third of respondents (106) reported themselves as knowledgeable or expert in computer security, and we used this group of respondents as our security experts. We classified all other participants as "non-experts" or security amateurs. We found a few differences in reported behaviours between the expert and non-expert groups, but it appeared that the experts relied on many of the same coping strategies as non-experts.

Security experts were less likely to have "go-to" password (44\% compared with $67 \%$ of amateurs). However, those experts who did use a primary password appeared to use

\footnotetext{
${ }^{2}$ Some respondents did not disclose their age group, so numbers do not add to the total number of respondents.
} 


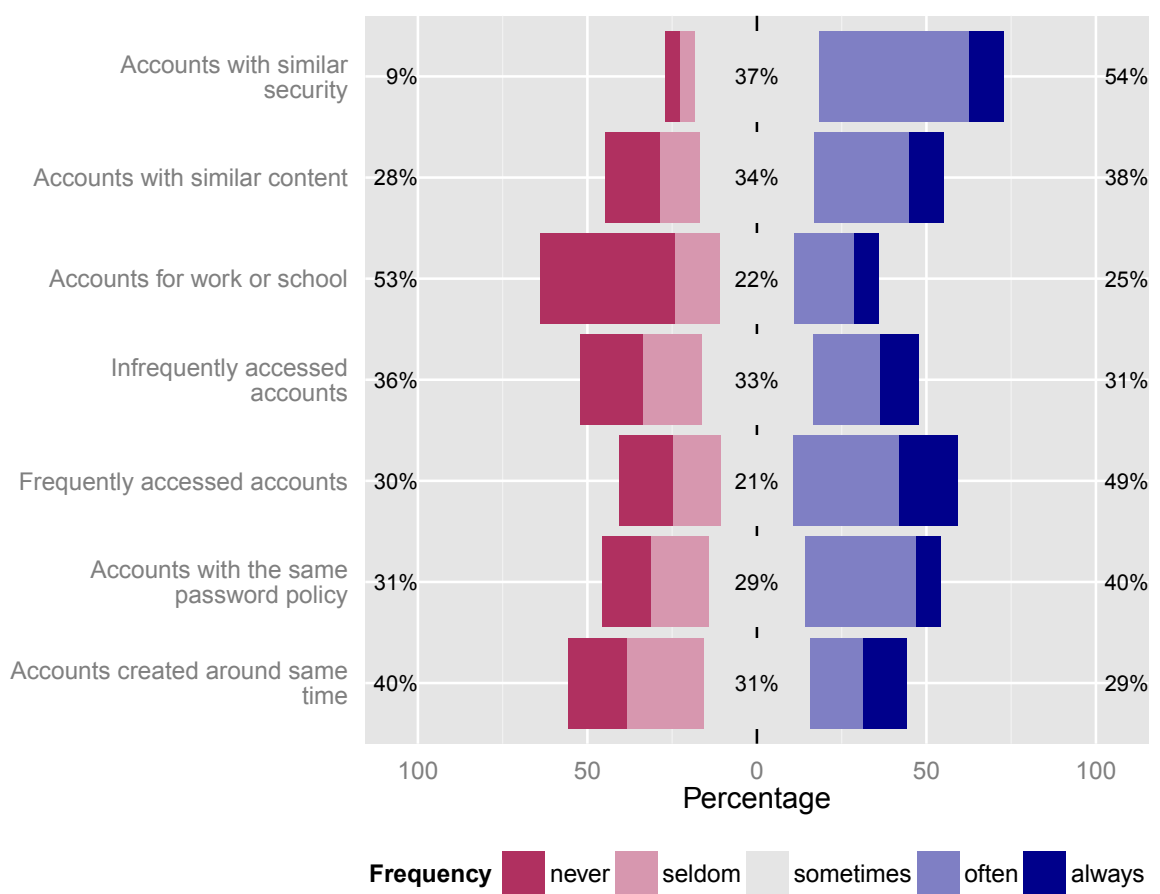

(a) Older users $(35+)$

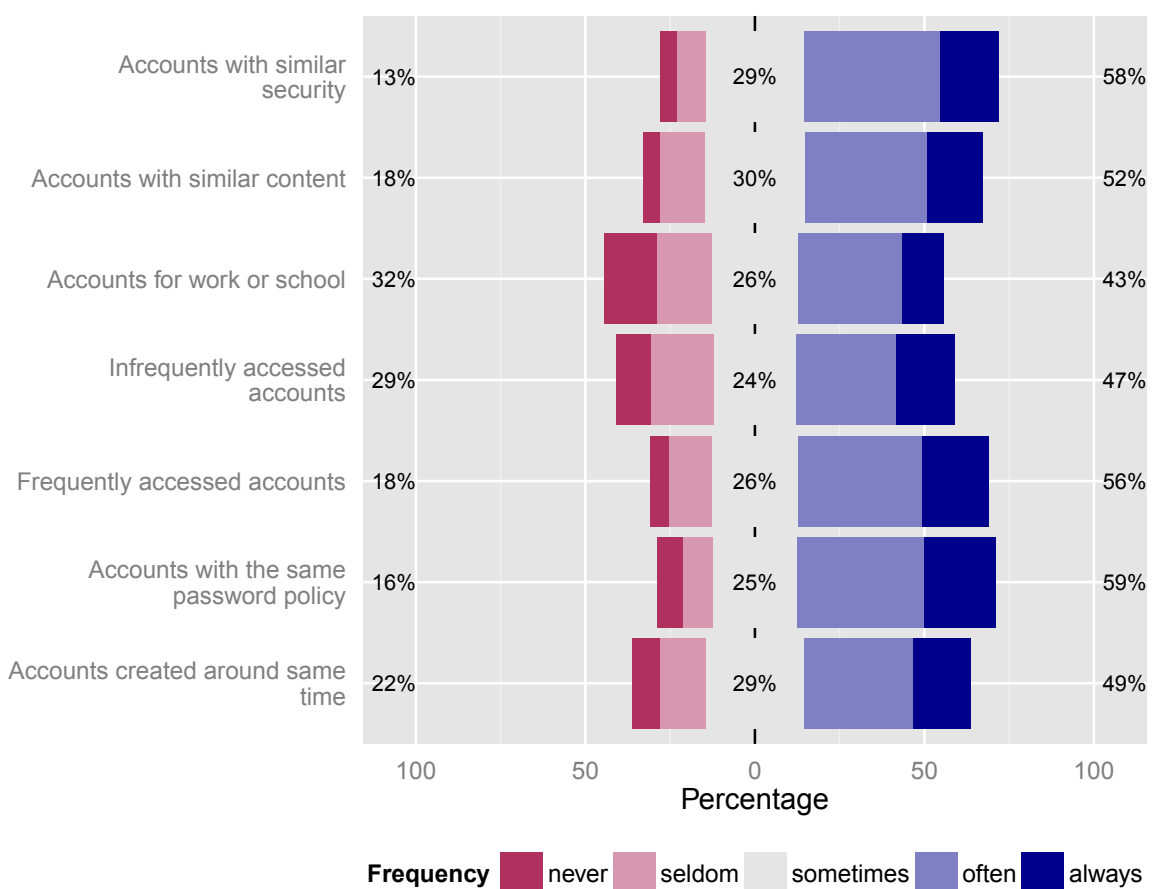

(b) Younger users $(<35)$

Figure 7.17: Rating scales showing how older and younger users reuse passwords. 


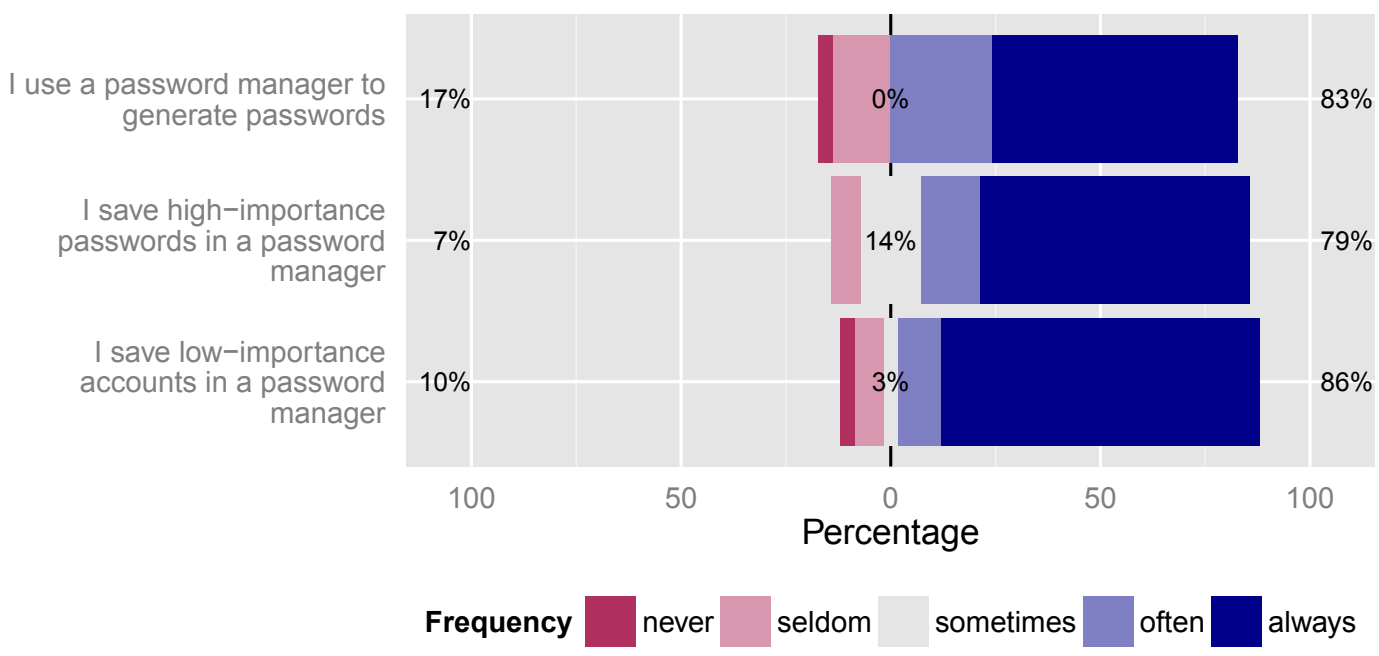

Figure 7.18: Rating scales showing how security experts use password managers.

it similarly to non-experts. Security experts were also less likely to reuse passwords ( $63 \%$ compared with $80 \%$ of amateur users), but when they did reuse passwords, they followed similar categorization strategies to non-experts.

Unsurprisingly, more security experts reported that they used a dedicated password manager ( $28 \%$ compared with $12 \%$ of non-experts). These expert users reported making more frequent use of password manager features, particularly to generate passwords (Figure 7.18). For those respondents who did not use a password manager, experts were most likely to attribute this non-use to trust issues. Non-experts cited more varied reasons, and were more likely to not know what a password manager is, and to not want to set one up (Figure 7.19).

Security experts were more likely to be in the habit of changing their passwords regularly (44\% versus 25\%). Aside from this difference, experts and non-experts seemed to have similar behaviour when changing and resetting passwords.

Interestingly, security perceptions were quite similar between non-experts and experts. Experts were more likely to think that they were doing a good job protecting their accounts ( $77 \%$ agreement compared with $59 \%$ for non-experts), and evidently felt more confident that they were doing all that they could to protect their accounts (72\% agreement that they were "doing their best" versus $56 \%$ ). 


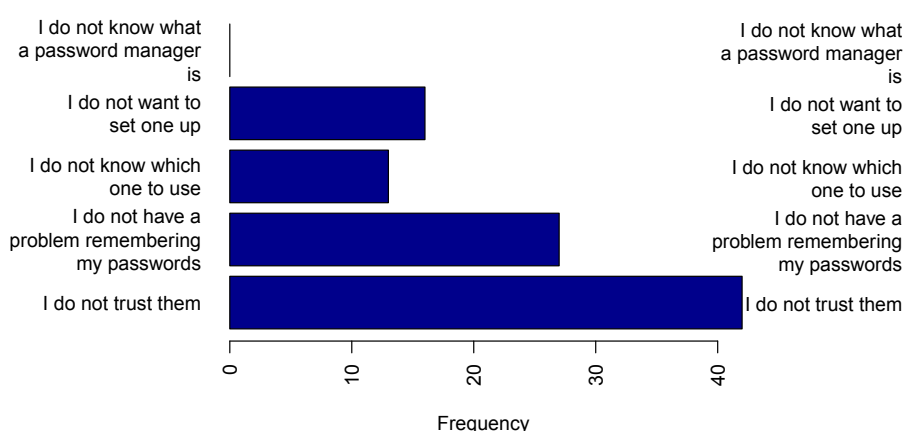

(a) Security experts

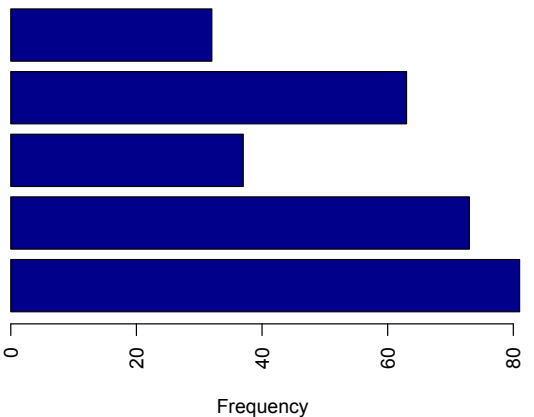

(b) Security non-experts

Figure 7.19: Barplots showing why experts and non-experts do not use password managers.

\subsection{Discussion}

Our work in Chapter 5 suggested that passwords are moved through a kind of life cycle, where they are first created, then saved, perhaps reused, and potentially forgotten. In our survey, we found evidence of consistent strategies for password creation and reuse, as well as evidence that passwords are cycled through different accounts and modified for different accounts.

Our survey showed evidence that respondents use consistent password creation strategies. Many respondents reported always or often including a specific type of personal information in passwords, and half of all participants reported using a consistent symbol, digit, or capitalized letter in their passwords. Although these strategies make passwords easier to remember, they may also make them vulnerable to attack if an attacker figures out the pattern. Many participants also said that they relied on a consistent password composition strategy, and the most popular combination of elements for these passwords were a particular word and a particular digit. Participants also reused their passwords consistently. The majority of survey respondents said they reused passwords, and predictably, gave easier memorability as their reason for reuse.

An important aspect of the life cycle is that even time passes, parts of passwords and habits are maintained. We found strong support for this: the majority of survey respondents reported having a primary password, and using it (or variations of it) on multiple accounts. Respondents said that they often included part of another pass- 
word in new passwords, and when they change passwords, they often maintain part of the previous or another existing password. Respondents also reported "retiring" passwords by creating a new password on a high-security account, and then reusing the old password on other accounts. Again, these strategies reduce cognitive load, but can make accounts more vulnerable to attack.

Our respondents seemed not to be very discouraged by the tasks of password management. The survey results showed that respondents thought that they were doing a good job protecting their accounts, did not have trouble keeping track of their passwords, and had time to pay attention to security. Most reported that they found it easy to pick new passwords. This is surprising to us, both because it differs from the impressions given in the interviews, and because it differs from the popular culture surrounding passwords. It is difficult to know why these survey results are different. One possibility is sampling bias; perhaps our survey description attracted respondents who felt attentive to and confident about security, thus skewing the results. It is also possible that respondents tried to tell us what they thought we wanted to hear (a variation of the Hawthorne effect). Another possibility is that this is a more accurate representation of attitudes towards passwords, and that the stories most often heard anecdotally are those of the proverbial "squeaky wheel".

\subsubsection{Rationing}

We found evidence of rationing in the survey results, but less strongly than we had expected. There was evidence that users supported the division of resources: most respondents agreed that they gave some accounts more attention than others, and that some accounts deserve this extra attention. However, participants did not agree that this attention was limited, and a large majority disagreed that they had problems deciding where to focus their security attention. This was somewhat counter to the impressions given of password management in the interviews.

Apart from respondents' perception of their own practice, it was clear that they did have different strategies for different accounts. Among respondents who reused passwords, many participants said that they did not reuse passwords on banking websites and online stores that saved credit card information. People clearly had different strategies for accounts with different financial significance. 
These results show that users are thoughtfully distributing their own efforts, but do not perceive it as such. It is positive that users do not seem to view this rationing as a hardship, but also seems to show a lack of awareness that could possibly have a negative impact of password management behaviours.

\subsubsection{Password Management Tools}

The results of our survey support our interview finding that users are not making good use of password tools. Fewer than one in five respondents used a dedicated password manager, and experts were far more likely to use a password than nonexperts. However, even users of password managers indicated that they were not taking full advantage of the password manager features (34\% of password manager users said that they did not use the manager to generate passwords). This seems to point to a usability problem with password managers.

Among non-experts, the most common reasons for not using a password manager were not knowing what a manager is, and an unwillingness to set one up. Among experts, lack of trust was the primary reason for not using a password manager. An obvious way to address this lack of trust would be to create an open-source password manager, making all sensitive operations auditable. However, open source password managers do exist (e.g., KeePassX), and have not entirely addressed this problem.

\subsubsection{Limitations}

Survey-based research has a number of possible limitations that may have affected our study. Mechanical Turk is an efficient and convenient method of recruiting participants, but it can be difficult to tell exactly who is completing the survey and whether they are telling the truth. Studies of demographics on Mechanical Turk have shown that samples recruited from Mechanical Turk are often more representative of the general population than in-person convenience samples [10]. However, it is possible that the population of workers on Mechanical Turk are more computer-literate than the general population. Our survey sample rated their computer expertise as 
high: two thirds of our sample reported their computer knowledge as knowledgeable or expert.

Sampling bias was another possible limitation of our study. Passwords are an obviously technical subject, and one concern is that the subject of the survey may have attracted participants with an existing interest in security, or even computers. This may have biased the results of the survey toward users who are more knowledgeable about and more attentive to security.

\subsection{Conclusion}

Our survey found evidence that a broader population relies on the same kinds of coping strategies for password management as our interview participants. Respondents in our survey reported having systematic ways of creating passwords, reusing passwords for convenience and memorability, and creating variations on a "go-to" password to use on different accounts. Respondents divide their attention among multiple accounts, and agree that some accounts deserve more attention than others. While these strategies minimize cognitive load and help users manage all of their accounts, they can also create security vulnerabilities for users.

The results of our survey show support for the life cycle model. Our respondents indicated that they do move their passwords through various steps, varying them for security while keeping common elements of them for memorability. Respondents also described rationing their efforts to different accounts. 


\section{Chapter 8}

\section{A Password Manager that Doesn't Remember Passwords}

\subsection{Introduction}

Although our earlier work addressed memorability issues for graphical passwords (Chapter 4), and developed design requirements for password management strategies (Chapters $5 \& 6$ ), it still remains to tie these ideas together into a practical solution for end users. We have developed Versipass, a password manager that incorporates key elements of password managers and cued graphical passwords to avoid existing problems of password memorability and associating passwords with accounts. Instead of remembering passwords, Versipass remembers image cues for graphical passwords. These cues help users to better remember their passwords and to more easily link passwords with accounts. Versipass also facilitates safe password reuse by allowing users to have the same image cue for multiple accounts. When a user attaches the same password cue to multiple accounts, Versipass produces a different hashed password for each account, leading to safer password reuse.

Alone, neither cued graphical passwords nor password managers completely solve users' problems with passwords. However, these ideas together provide support for the problems that users commonly have with passwords. They provide better memorability, while better organizing users' passwords and the associations between passwords and accounts. 
In this chapter, we introduce Versipass, and describe our cue and category models that support users in coping with password management. We describe the Versipass prototype, and analyze its security. We present the results of an early usability evaluation, and discuss how Versipass's properties might fit into the landscape of future password management.

\subsection{Password Coping Techniques}

Our research on graphical passwords has shown that assigned graphical passwords are indeed more memorable than assigned text passwords (see Chapter 4), and that the image cues associated with some graphical passwords can help users distinguish multiple passwords from each other [30]. In particular, we have found that random assigned Image PassTiles passwords are more memorable than other assigned passwords, as well as being easy to use and less susceptible to multiple password interference. However, though Image PassTiles passwords are more memorable than comparable text passwords, users are still unable to remember as many of them as they have accounts.

Password managers are potentially one of the best existing technologies to help the end user handle knowledge-based authentication. Password managers are programs that save or generate and enter passwords for the user, and have an advantage over technologies like single sign-on (SSO) because the user may choose to use them on any account (instead of waiting for the account administrators to implement SSO). Password managers address the issue of quantity because they are able to remember any number of passwords.

Password managers are not without problems. Both hashing and wallet password managers have a single point of failure in the master password (see Section 2.4.3). If this password is revealed, an attacker can gain access to all of a user's accounts. Password managers may also be incautious about how the passwords are stored: some of the browser-based password managers use a wallet model, but store users' passwords in the clear [61]. Another issue for password managers is roaming: password managers are typically difficult to transport to other computers and browsers, and can complicate life for users who use a variety of computers or devices. 

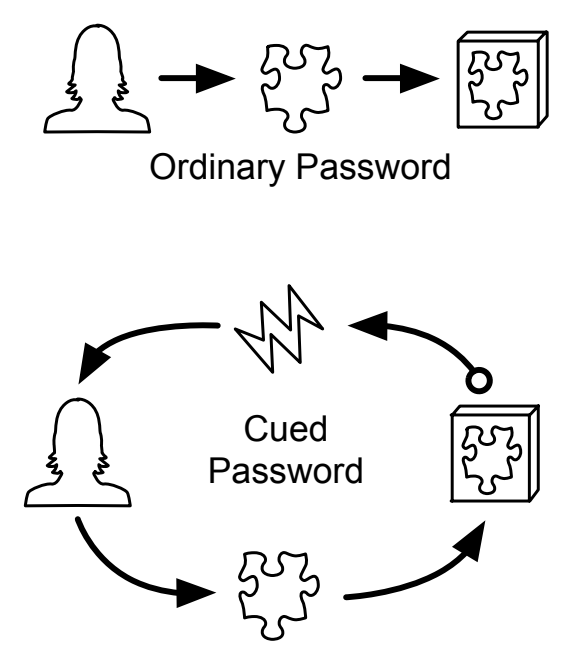

Figure 8.1: The cue model. The account provides a password cue to the user, who uses it to associate and remember their password.

\subsection{A New Model for Passwords}

Versipass incorporates two key ideas to address the issues of cueing passwords and password reuse. Although these ideas are used elsewhere, Versipass is the first system to combine them, and we believe that the combination of these ideas gives more benefit to the user than either idea alone.

\subsubsection{The Cue Model}

Versipass is more rightly a password cue manager, since it does not actually store any passwords. Instead, it stores password cues that allow users to generate passwords and safely pass them to websites.

In the cue model (Figure 8.1), the password system sends a cue to the user, who uses it to recall their password. This model is used in cued graphical passwords and in other cued passwords, as well as by Versipass. More generally, the cue model describes all cued passwords. The cue may be visual, or auditory, but its purpose is to cue memory and provide context for the user. Another example of cued passwords are challenge questions, often used for password resets (challenge questions are discussed in Section 2.4.5). 

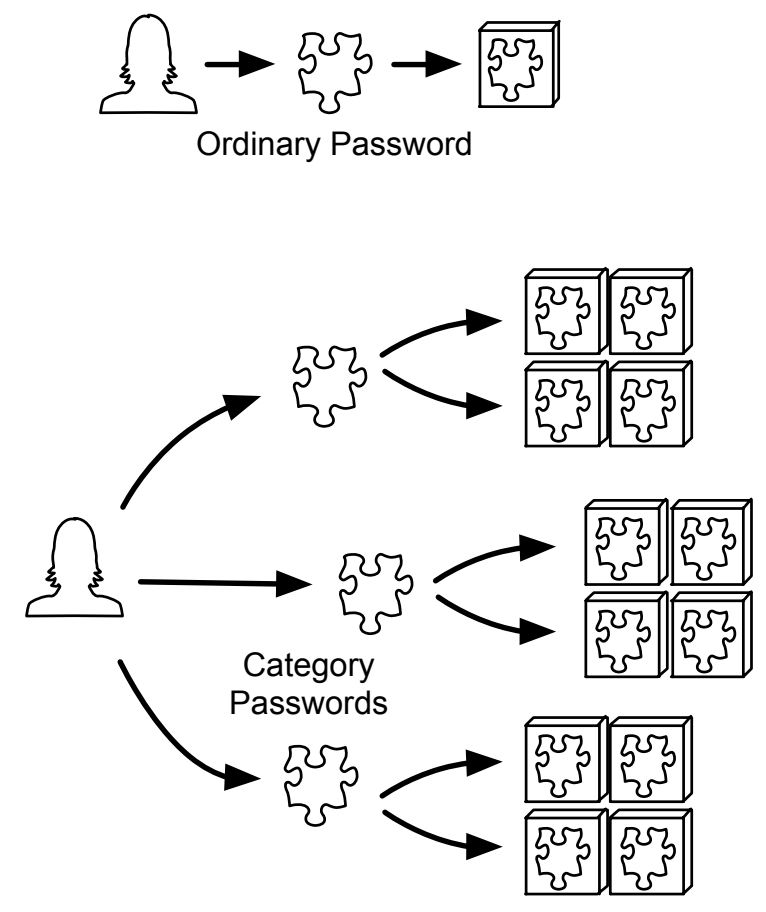

Figure 8.2: The category model. A user has a password for each category of accounts.

\subsubsection{The Category Model}

Figure 8.2 shows how category passwords are used to protect multiple accounts in Versipass. A single password is assigned to a category of accounts, and we expect that a user might categorize accounts with a similar theme, or that are used at a similar time. Although not portrayed in the figure, the password that is reused on multiple websites is salted and hashed differently for each of the websites where it is used. However, this step is invisible to users, much the way salting and hashing within websites is currently invisible to users.

Salted and hashed category passwords allow users to more safely reuse passwords across accounts. Password reuse is a key coping strategy for users, and one they are unlikely to abandon. If users are able to remember a small number of strong (randomly assigned) graphical passwords, then category passwords can allow them to apply those passwords to all of their accounts. While it would be possible for a user to group all of their accounts under one category, using multiple category passwords provides an extra layer of isolation for accounts in other categories if a category password is guessed. 
Through the use of the cue and category models, Versipass supports users' existing habits, while simultaneously protecting users against the risks of those habits. Since Versipass hashes the input responses differently for each website, it provides protection for users who reuse passwords. This allows passwords to be reused more safely while not increasing the burden on the user. Versipass also provides explicit cues, which help users to distinguish passwords and accounts. There is evidence that users already look for cues in the webpage environs, but Versipass provides a strong cue that is present at password creation to help users remember their passwords, and associate them with the correct accounts. Cues also minimize the possibility that users will systematically guess all of their passwords on every website, a coping strategy that can expose all of a user's passwords to an attacker in a single session.

The category model builds on the work of existing hashing password managers. PwdHash is a password manager developed by Ross et al. [110] that hashes a user's master password together with the website domain name and an optional salt to create unique passwords for every website. However, PwdHash uses the same password for all accounts, and does not include the notion of grouped accounts under a single password category. Versipass introduces cues to support random assigned passwords, and allows users to protect accounts in different categories, avoiding a single point of failure.

Another advantage of Versipass is that it allows graphical passwords to be used on any website without requiring any server-side changes. Password schemes are implemented through Versipass, and require no changes to existing website infrastructure. This means that users can choose the type of password with which they are most comfortable.

\subsection{Versipass Prototype}

In this section, we present the current Versipass prototype. Versipass makes use of the Image PassTiles graphical password scheme. We briefly describe Image PassTiles here, but more details can be found in Chapter 3. In this section, we also present walkthroughs of the Versipass prototype and a discussion of the issues facing a future implementation of Versipass. 


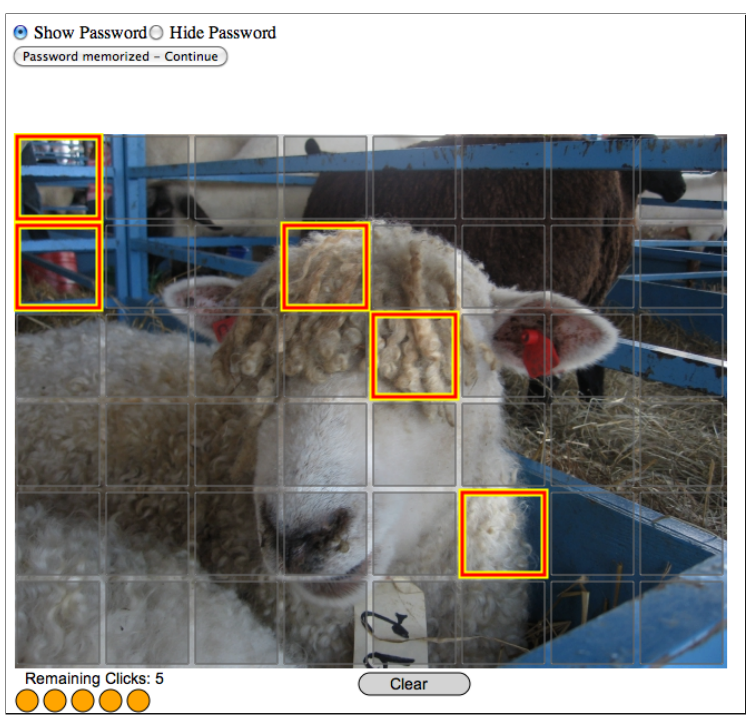

Figure 8.3: The password creation interface for Image PassTiles. The tiles highlighted in orange are the user's assigned password tiles.

\subsubsection{Image PassTiles}

Image PassTiles is a variation of PassTiles that presents the user with an image overlain by a grid (Figure 8.3), and the user's password consists of a set of grid tiles. To log in, the user must simply click on each of their password tiles, in any order. In this work, we used the standard configuration, of a grid with 48 tiles $(6 \times 8)$, and passwords with 5 tiles. The passwords are randomly assigned: they are generated by the system, and the user is given no choice about which tiles are included in their password. Because the passwords are randomly generated, they are not related to the image cues, and no information can be inferred from the images by themselves. The background images can be any images, and could even be the user's personal images. Image PassTiles passwords are encoded as text strings by labelling each password tile with a string, and then concatenating the string of clicked tiles. Since password tiles may be clicked by the user in any order, the text string is then given a standard ordering for comparison. The encoded text strings can be treated as any other text passwords, and can be salted and hashed for secure storage. 


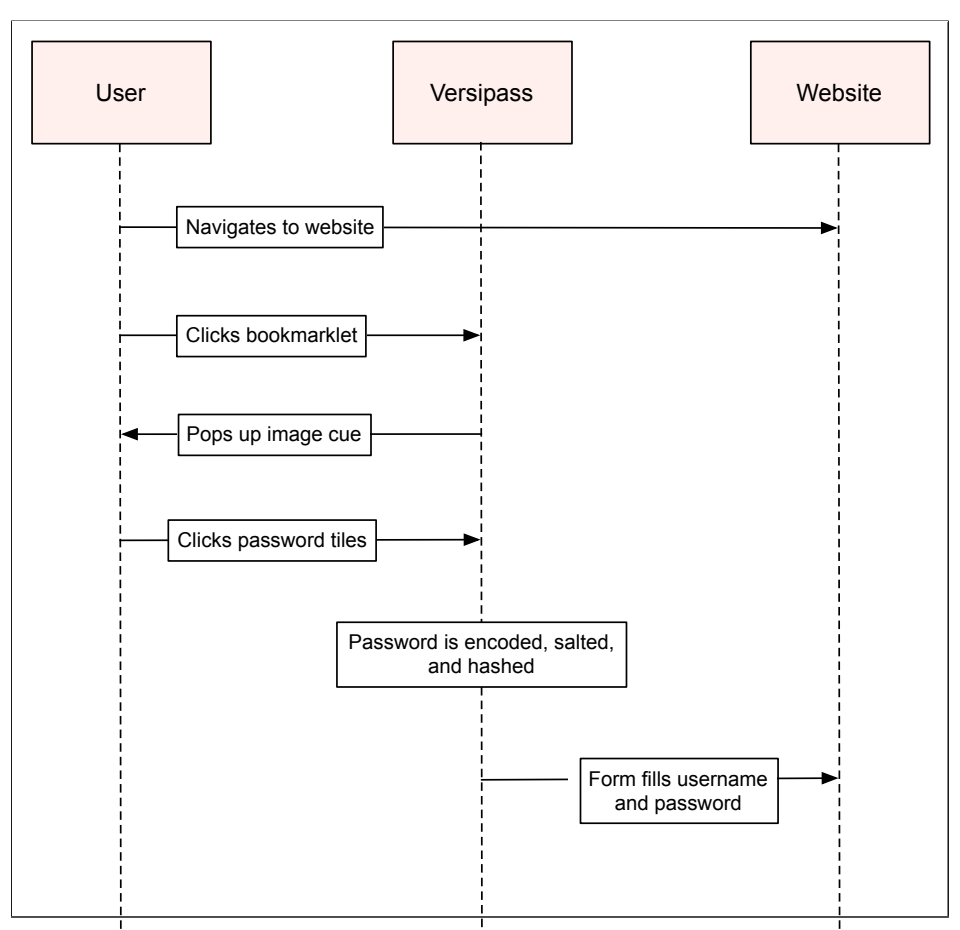

Figure 8.4: A sequence diagram of the login process using Versipass.

\subsubsection{The Versipass Password Manager}

The current prototype of Versipass is built to integrate with MVP [27], a framework we constructed for earlier research to allow us to conduct ecologically valid tests of password systems. The Versipass prototype draws its implementation of Image PassTiles from MVP, and saves user account details in the MVP database. The prototype is implemented as a web application that the user must sign into to set up their passwords and accounts. To use Versipass when logging in to websites, we have provided a bookmarklet for the user to save. The bookmarklet is a short piece of JavaScript code that relays the userID and URL of the site back to MVP to look up the saved information for that account. Figure 8.4 shows a sequence diagram of a login using Versipass.

Figure 8.5 shows screenshots of the step-by-step process to add a new website account and password to Versipass, and Figure 8.6 shows the step-by-step process that a user would take to log into a website using Versipass. In both walkthroughs, the scenario begins with the user already logged into the password manager. The user does not need to have the Versipass webpage open, but they do need to log in to Versipass at the beginning of every session (the login information is saved in a session cookie). 


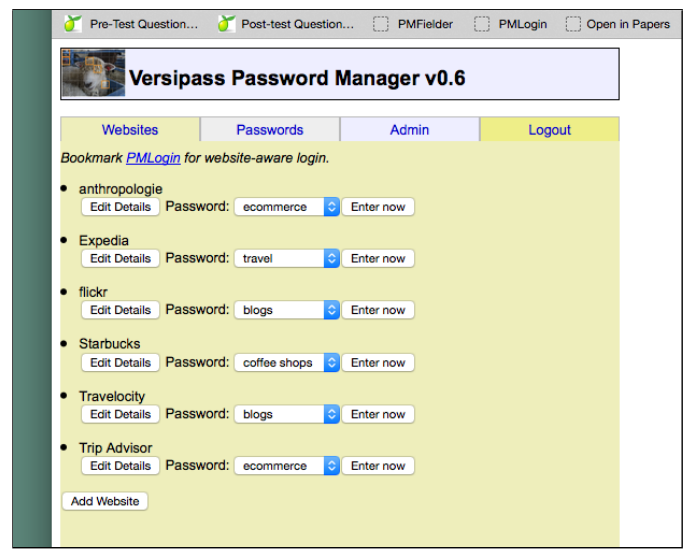

(a) To add a new website account, the user clicks "Add Website" on the Website tab.

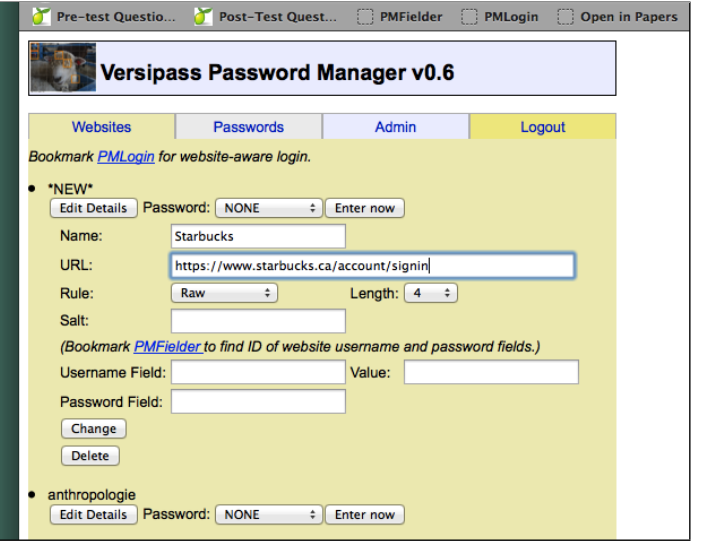

(c) The user fills in the name of the website, and refers to the website itself to find the URL of the sign-in page.

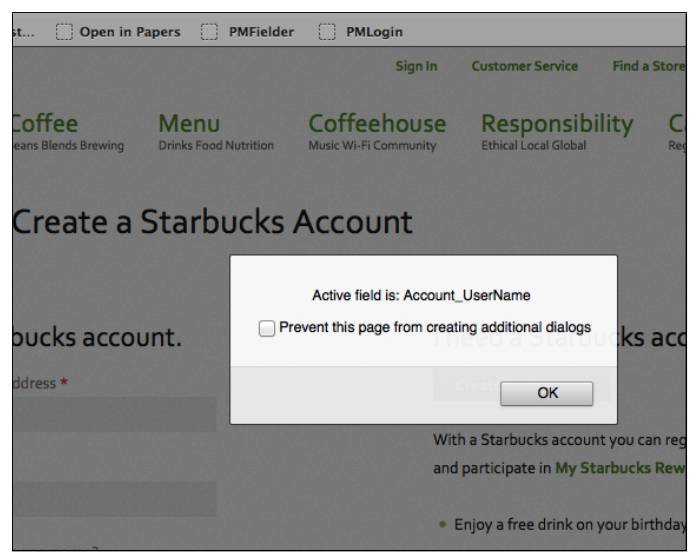

(e) The user saves the PM Fielder bookmarklet. On the website, the user clicks into the relevant field, and then clicks on the bookmarklet to pop up the HTML field ID.

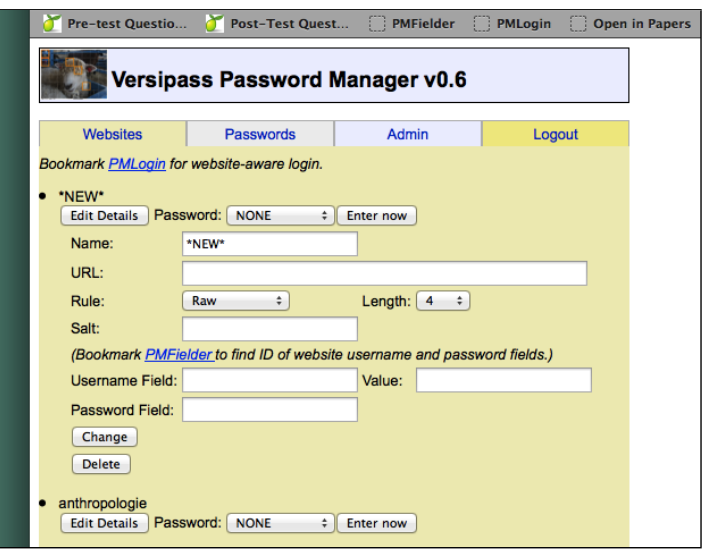

(b) The user clicks "Edit Details" to set up the new account.

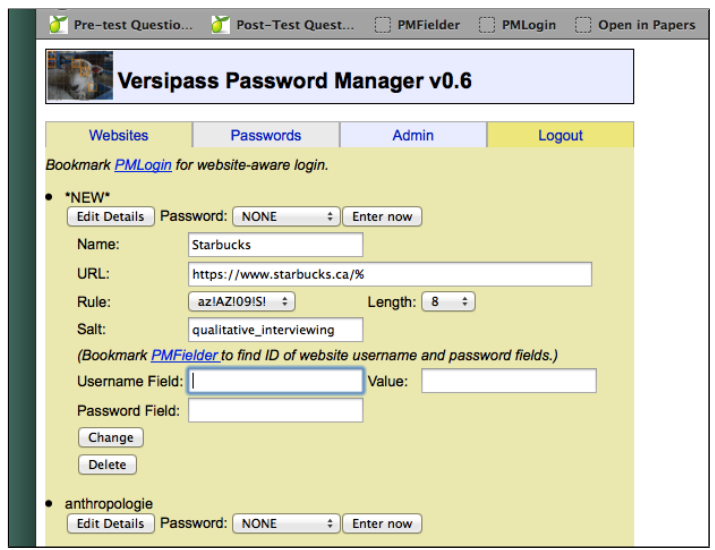

(d) The user chooses a password rule and length from drop-down menus, and inputs a user-chosen salt. The salt can be any string, and does not need to be a secret.

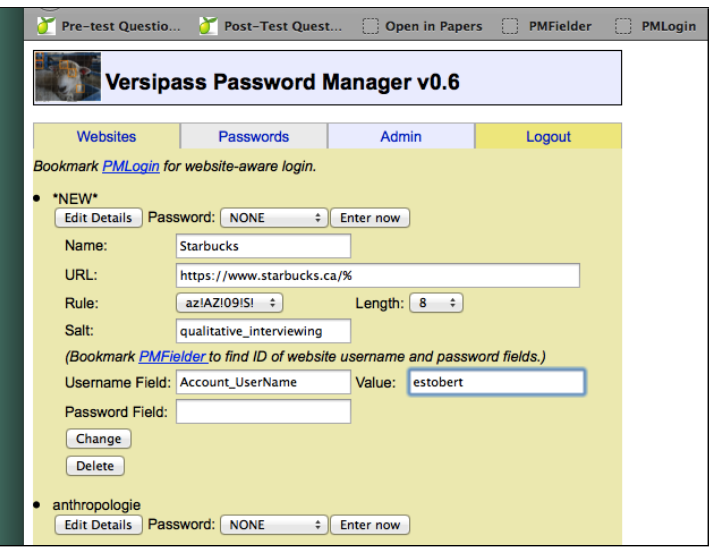

(f) Back in Versipass, the field ID is copied into the username box, and the value is filled in with the actual username.

Figure 8.5: Adding a new account and password category to Versipass (continued on next page). 


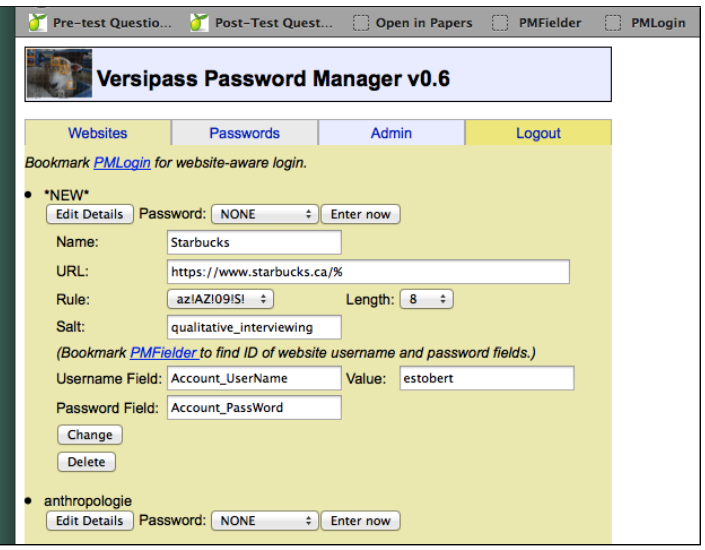

(g) The user follows the same procedure to find the HTML field ID for the password field.

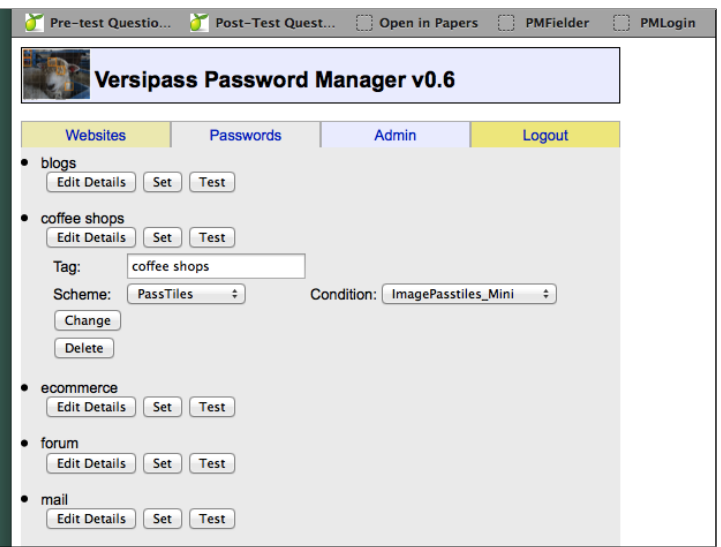

(i) The user gives the category a tag name and chooses a password scheme (and condition, if applicable).

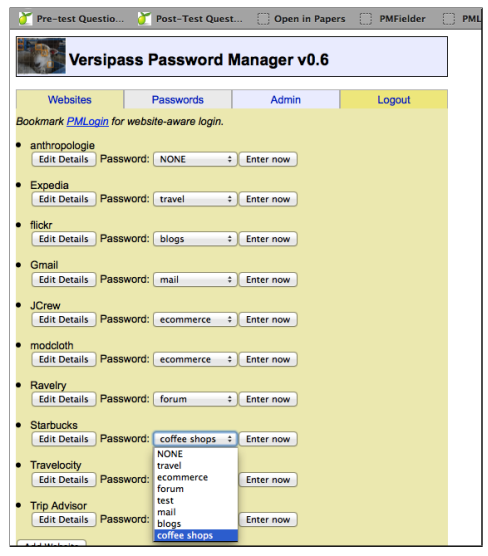

(k) In the Websites tab, the drop-down menu is used to link the "coffee shops" password to the Starbucks website account.

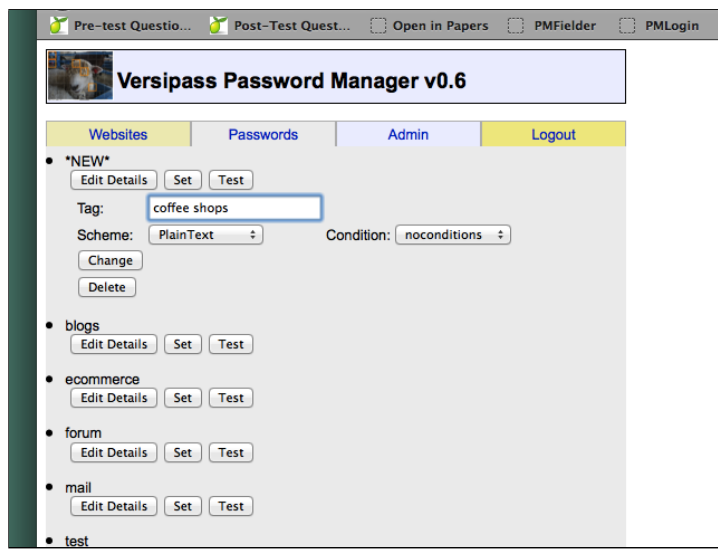

(h) In the Passwords tab, the user clicks the "Add Password" button to add a new category, and clicks "Edit Details" to configure the category.

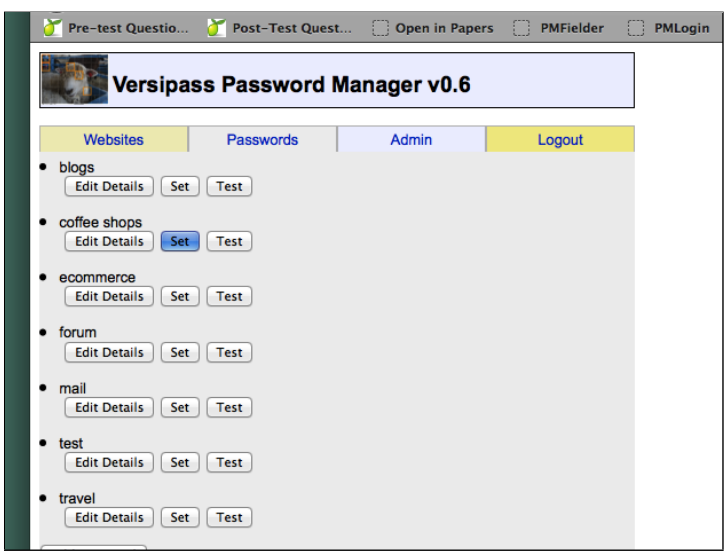

(j) Next, the user attaches a password to the category. The user presses "Set" to set up a password of the type already selected.

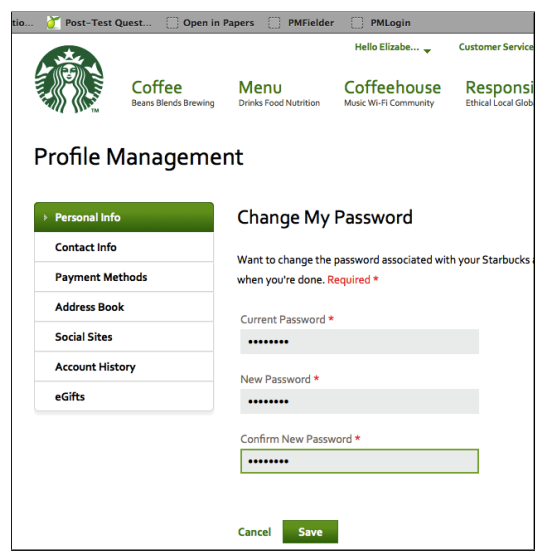

(l) The user must change the password on the Starbucks website to match the password that is generated by Versipass.

Figure 8.5: (cont.) Adding a new account and password category to Versipass. 


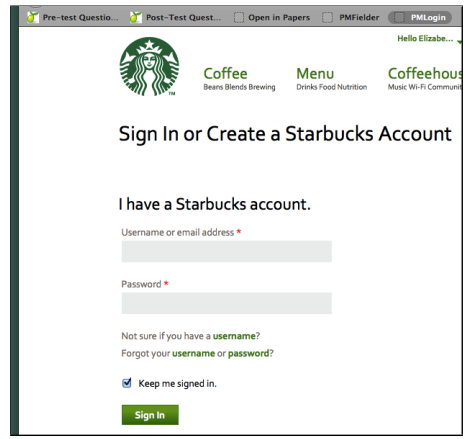

(a) The user clicks the PMLogin bookmarklet from the website login page.

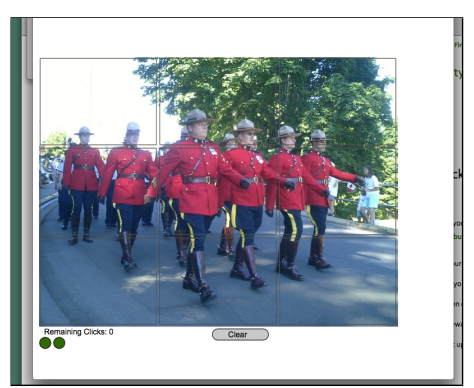

(b) The bookmarklet pops up the image cue, and the user enters their response.

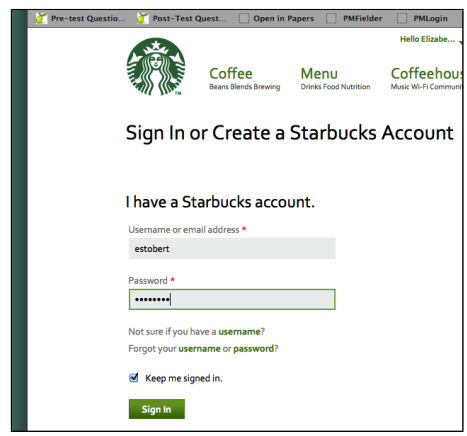

(c) Versipass generates the password string, and pastes the username and password into the appropriate fields.

Figure 8.6: Logging in using Versipass.

\section{Future Implementation Considerations}

Our current implementation of Versipass was constructed as a proof of concept, and many of our design decisions were based on the convenience of using our existing infrastructure. However, a real implementation of Versipass would need to carefully consider security issues relating to the secure transmission of data, and avoid design decisions that limit security.

We initially chose the bookmarklet approach for its flexibility, considering that users would be able to save the bookmarklet on any computer, even where they did not have access to system settings. However, we now think that a better approach would be a browser extension. Although the extension would have to be installed by the user, it would avoid issues with copying and pasting the password from one browser tab (or window) to another that the current bookmarklet approach has had to work around. We plan to implement future prototypes of Versipass as browser extensions.

Ross et al. [110] identify a number of challenges affecting the implementation of a browser extension for their password manager, PwdHash. These issues include JavaScript attacks on the cleartext password, salting, encoding the password to fit website password policies, compliance with browser auto-complete functionality, helping the user with password resets, roaming across different computers, and dictionary attacks. We address the issues relating to implemention in this section, and discuss the issues relating to Versipass' security in Section 8.5. 
Versipass uses salts to protect the cleartext passwords, and lets users select part of their own salts. In our prototype, the user provides a string that is used together with the URL of the website as the salt for that account. We took this approach because situations arise where users need to change their passwords, and a simple way to do this is to change the salt. Simply using the URL would mean that there is no way to change the salt for an account. We have considered creating a mechanism to randomly generate salts, but this could be dangerous if a user changed the salt without understanding the effect on the generated password and effectively locked themselves out of their accounts (even if they knew the password input). One way to mitigate this problem might be to keep a list of previously used salts, allowing the user to revert to earlier salts.

Versipass addresses the issue of encoding passwords to comply with password policies by having the user select the length and policy for the generated password. However, password policies are often not explicitly displayed, or only explained when they are not followed, so this might pose a problem for users. An improvement to Versipass might be the automation of this step, either through crowdsourcing (password policies for a website could be saved for all users of Versipass), or through some means (e.g., a password API) of allowing Versipass to automatically learn the password policy.

In its current implementation as a webpage with bookmarklets, Versipass can be used on any computer without any installation. This has a definite usability advantage to a user who uses multiple computers and does not have installation privileges on those computers, but may not be advantageous to all users. The bookmarklet results in a complicated user interface because of the implementation difficulties of passing information between multiple browser tabs. Implementing Versipass as a browser extension will limit where Versipass can be used, but hopefully improve its usability when it is installed.

\subsection{Versipass Security}

In this security analysis, we address the security of the Versipass password manager. It should also be noted that Versipass's security is affected by the security of Image PassTiles, which is discussed in Section 3.5. 
Since Versipass does not store passwords, there is no risk that an attacker who gains access to Versipass might be able to access the user's accounts. However, such an attacker would be able to access personal information (the list of sites where the user has accounts, and the user's usernames), and cause significant disruption for the user (by changing their category passwords or by deleting information). Users should still be able to access their accounts via emailed password resets or challenge questions, but this kind of attack could be very frustrating for users. One possibility is that Versipass could provide a mechanism for users to create offline backups that could be later restored in the case of an attack.

Versipass protects against phishing attacks in the same way that other password managers do. If the URL of a login page is unknown to Versipass, the bookmarklet will not work, and Versipass will not pop up the image cue, or generate (and form-fill) a password for the site. A useful addition might be a warning message that alerts the user that they might be trying to log into a fraudulent website.

In the current form, Versipass stores all of the user's information on a server. This means that the user can access Versipass from any computer, and have access to their accounts no matter where they are. Even if the user does not have the ability to save bookmarks, they can still generate their passwords in the Versipass web page and copy them over to the websites manually. However, this also means that if the server is attacked (for example, in a denial-of-service (DoS) attack), the user might not be able to $\log$ into any of their accounts. If the Versipass database was stored locally (for example, with a browser extension), the user would be less vulnerable to DoS attacks, but would have to transfer that database in order to be able to use multiple computers.

Another consideration resulting from Versipass's server-side implementation is that of secure communication. To ensure that an attacker cannot eavesdrop on communication between the client and server, all communication should be encrypted using SSL.

Although it is not the way that two-factor authentication is usually designed, Versipass provides a second layer of authentication in addition to what is provided on the website. Because the user must be signed in to Versipass to access their password cues and generation, they will have provided two layers of authentication any time they use Versipass to log into a website. It is also possible that the Versipass 
authentication could incorporate an additional authentication factor. For example, authentication to Versipass might happen via a one-time code that is provided over SMS.

Since Versipass only operates on the password entry portion of website logins, it should not interfere with existing two-factor authentication schemes. As long as the user still has access to the second factor, Versipass should not complicate the twofactor authentication.

\subsection{Usability Evaluation}

Versipass is a new paradigm for the end user, and as such, it is important that the password manager interface should be usable and comprehensible for users. Specific usability testing is needed to evaluate the new concepts within Versipass (password categories, no stored passwords, etc.), as well as the PassTiles passwords. Because Versipass's intended purpose is to help the end user behave more securely, we felt it was important to conduct early evaluations of Versipass's usability. These evaluations were intended to help us understand users' impressions of the system and to inform future iterations of the design.

We conducted two evaluations of Versipass: a preliminary user study, and a cognitive walkthrough. Our user study was conducted with five participants from our lab (while familiar with our work, these participants were not security researchers or experts in security), and was intended to give an initial impression of the usability of the system. No personal data was collected, and the participants were fully informed about the purpose and intent of the study. We subsequently conducted a cognitive walkthrough to give more detail about the problems found in the user study, and to allow us to better understand the problems.

Since the two evaluations yielded similar results, we present the combined results of the two evaluations in Section 8.6.2. We first present the separate methodologies, and then the combined results. 


\subsubsection{Method}

\section{User Study}

In our user study, participants were given a scenario and a few tasks and asked to think aloud as they completed the tasks. We gave participants a brief explanation about the purpose of the study, and told participants that they should be honest about their impressions of the system since we were evaluating the system, not their performance. Participants began by completing a short pre-test questionnaire that collected information about the participant's security habits and knowledge. They were given a written scenario describing a persona, Jo Kelly, who had begun a new job as a travel blogger and wanted to manage her multiple accounts using the password manager. They were also given index cards with Jo's account information. At the end of the scenario description, participants were asked to complete four tasks which included logging in to Versipass, adding a new account to Versipass, logging in to that account using Versipass, and configuring Versipass to use the same password on multiple websites.

Participants were asked to "think aloud", describing their actions and thought processes as they worked through the task list. In the case that the participant became confused by a task unrelated to the password manager, the experimenter provided help in navigation. For instance, participants had to interact minimally with a few external websites, and one participant had difficulty finding a login link on a website.

Four participants reported that they use some kind of password manager in real life. Three of these reported using the password manager built into the Firefox web browser, and the remaining participant reported using LastPass.

\section{Cognitive Walkthrough}

In our second evaluation, we conducted a cognitive walkthrough to follow up on the results of the user study, and to help us better understand where the problems that arose in the user study were rooted. A cognitive walkthrough is an inspection method evaluation that focuses on learnability for new users [143]. A group of evaluators steps through several tasks, and evaluates a persona's problem solving process at each step. At every step, three questions are asked: Will the user know what to do?, Will the 
user see how to do it?, and after they have completed the action, Will the user know that they did the right thing?

We conducted a pluralistic walkthrough, in which multiple evaluators came together to assess the usability of the prototype. The participants in our cognitive walkthrough were the author of this thesis and her supervisor, who designed and constructed the prototype under evaluation. The third participant was another graduate student in our department, who was familiar with usability and security, but who was not specifically knowledgeable about password managers or familiar with our prototype or design. We conducted the evaluation using the prototype described in Section 8.4. We projected it onto a large screen in our lab to be equally visible to all participants, and each participant took on a role in the walkthrough. The roles were operator/navigator, notetaker, and persona.

For our cognitive walkthrough we used the same persona and tasks as in the user study. We assumed that Jo had already done some initial setup of the password manager. She had an existing Versipass account with credentials, and had already added a few websites and passwords.

\subsubsection{Results}

In general, participants in the user study had difficulty with the password manager tasks, and we identified several ways in which our interface might be difficult to learn. Participants had differing levels of success, but none of the participants was able to successfully complete all of the tasks. The results of our evaluations identified three types of problems: mental model problems, security issues, and incorrect interface elements.

\section{Mental Model Problems}

Many of the errors that users made while completing the tasks seemed to be caused by a lack of understanding of how Versipass worked. In the cognitive walkthrough, although we had deep knowledge of the system, we still found ourselves misled based on a lack of awareness of where we were in the sequence of events. 
In the user study, participants were not given any explanation of how Versipass worked, and although most participants eventually figured out how the manager worked, most of them missed the nuances of the functions, and misunderstood the advantages given by Versipass. Participants were initially confused by the separation of website accounts from password accounts. Most participants figured out this functionality, but they did not understand that using the same password cue on different website entries could mean sending different passwords to different accounts. The same result was echoed in the cognitive walkthrough, where we observed that it might be possible for a user to make their way through the set up process without really understanding the given advantages, or how Versipass differs from other password managers.

Since users did not necessarily understand that Versipass is designed to encode input passwords and hash them before passing them to the websites, several participants in our study were confused about why they would choose to use Versipass. In the words of one participant "if I enter the same password in the [password manager] [as I would enter on the website], why bother?". One of the major impediments to usability that we observed in the cognitive walkthrough was that the passwords on actual websites need to be changed to match the passwords generated by the password manager. This process is both tedious and confusing, since the user must login to the website with their existing credentials, use the password manager to generate a new password, change the website password to the generated password, and then configure the password manager for automated login.

A key problem that surfaced in the cognitive walkthrough was error recovery. We managed to make a serious error when we pasted the incorrect password into a password field on an actual website. Once this password was saved, we had no means of accessing the account except via the emailed reset. This incident emphasized the need for an error recovery strategy. Our mistake was caused by a few factors: a misunderstanding of which information was meant to be copied into which field, and the starred-out characters in the password field on the website prevented us from noticing the error until it was too late. Error recovery is a recurring issue for security products: it can be difficult to help users recover from an error without revealing key information, and it can also be difficult to know how much information was breached while the error was being made. The lack of mental model only worsens the problem, since the user may not even understand when they make errors. 


\section{Security Issues}

The second task (adding an email account to Versipass) in the user study was designed to highlight how participants would protect an account with high security value. This goal was not explicitly stated, and in general, we did not emphasize the realism of the study, but it was interesting that only one participant commented at all on the security of Versipass. This person stated that they would have liked to protect their email account, but did not understand how to go about doing so. Other participants made no mention of security at all, which was surprising in a study that primed users to pay particular attention to a tool for managing computer security. Participants clearly did not consider how Versipass might help them to increase the security of their accounts, meaning that the manager failed in one of its primary purposes.

\section{Incorrect Interface Elements}

Several aspects of Versipass's user interface were found to be misleading to users in the study. While these kinds of errors can be relatively easily addressed, they were a major impediment to the usability of the system, especially on the scale that they occurred. In the cognitive walkthrough, we flagged similar issues, and discussed specific fixes for many problems.

There were several places where the names of buttons and text entry fields confused participants, causing them to incorrectly enter information, enter wrong information, or lose information. In the accounts tab, the button that participants expected to be called "Save" was actually labelled "Change". Multiple participants mentioned looking for a save button, and more than once, participants neglected to click the "Change" button, leading to information loss. Also in the account management tab, text fields were provided where the user was meant to input the HTML labels of the username and password on the actual website login page. Participants consistently misunderstood what information was supposed to be put in these boxes, and several participants put their own usernames and passwords in these boxes. This is a dangerous error, since they were typing actual passwords in cleartext. Another example of poor labelling was a set of drop-down menus that changed the parameters of the hashed password. Participants did not understand the meaning of the labels "Rule", "Length", or "Salt", and were unable to divine their meaning from the information 
in the menus. As such, they were not able to use these features of the password manager.

As participants explored Versipass, there were a few key elements that participants consistently missed or ignored. The major example of this was the link to the PM Fielder bookmark. The link to the bookmarklet was contained in an explanatory sentence above the relevant fields. Strangely, although it was close to fields where participants were having trouble, participants consistently failed to read this sentence. One participant commented that they had disregarded the sentence because it began with the word "bookmark", and they associated that instruction with non-critical information. Of participants who eventually noticed the instruction, one participant was able to successfully follow the directions after clicking the link, but another participant appeared bewildered by the short instructions and made no attempt to follow them or even read them closely.

Setting up an account with Versipass necessarily involves a certain amount of interaction with a website because the website password must be changed to be the same as the password generated by Versipass. During our cognitive walkthrough, we realized that the interaction with the website needs to be carefully guided, and that the order in which actions should be completed also needs to be carefully indicated. For instance, the new account tab in Versipass should tell the user where the next step should happen, and what they should be trying to accomplish on the website.

Another place in which Versipass could better guide the user is the order in which the user should fill fields in the password manager. Although creating a website account in Versipass requires the same information as it would without Versipass (for instance, choosing the character sets used in the password and the length of the password), these steps are made more explicit in Versipass, and appear at a point in the process where the user may not have yet considered them. In our walkthrough, we realized that the fields in the new account tab had to be filled in out of order, causing confusion.

Many of the usability problems in the prototype are related to the nature of an early prototype. This prototype was constructed as a proof of concept and as an early exploration into the feasibility of the password manager idea, and we did not pay particular attention to style or user interaction. The usability issues with our prototype are a sobering reminder that these kinds of problems cannot be glossed 
over. We need to devote time and effort to producing an interface that is consistent, attractive, and user-friendly.

\subsection{Discussion}

Versipass presents a new paradigm for password management. By integrating features of graphical passwords and password managers into the cue and category models, Versipass is a system capable of helping users better manage their passwords by taking advantage of their innate capabilities and existing habits. Our evaluation reveals a number of challenges, relevant not only to Versipass, but also to password management.

\subsubsection{Mental Models of Versipass}

One of our observations in our evaluations was that a user might be able to configure and use Versipass without properly understanding the details of how Versipass manages passwords. This raised questions about how much the user needs to understand in order to use the password manager safely and successfully. More generally, how much do users need to understand about passwords and attacks to secure themselves online?

In our previous work on graphical passwords, participants have rarely seemed bothered by the idea of an image cue. Perhaps surprisingly, they seemed not to question the presence of the image on the site. Even in online studies where the experimenter was not present, participants appeared comfortable with the graphical password model. However, in the evaluations of Versipass, it seemed that users were confused by the presence of a cue that did not come from the website itself. We speculate that users misunderstand the cue model in the context of a password manager because of their preconceived idea that all password managers will remember passwords for you.

We intended that the concept of category passwords would allow users to strategically reuse passwords, much in the way that they currently reuse passwords. Our usability 
evaluation did not ask users how they would categorize their own passwords, but in earlier work [125] we had users sort a set of accounts into categories based on a shared password, and we found that all participants were able to easily separate a set of 80 accounts into five or fewer categories. Participants in that study used a variety of sorting criteria, including semantic, temporal, and security-based criteria. This result gave us confidence that the category model used in Versipass could work for users. In our Versipass study, participants seemed to understand that the same password cue could be used for multiple accounts. However, participants in our study did not understand that those reused passwords were encoded differently for each account. Although users do not need to understand the details of how the encoding differs, we do think that they need to be more aware that they are protecting each account.

A result of our cognitive walkthrough was that category passwords might be difficult for new users to set up from scratch. We thought that an example category (with a random password) could be added to new Versipass accounts to give users a starting point for configuring the manager.

Although we call Versipass a password manager, it actually works in a way that is fairly unlike the most existing password managers (of course, users don't understand existing managers very well either). We want users to understand how Versipass can support their existing habits, and keep them more secure online. To do this, users need to understand Versipass well enough to use the features as intended, and to avoid making dangerous mistakes.

To address some of the misconceptions surrounding Versipass, we propose changing its title, and positioning it as a login manager rather than a password manager. Versipass effectively manages the login process: while using Versipass, a user would rely on it to manage usernames and passwords, and as a means of accessing websites. Hopefully, removing the "password manager" title would stop users from making incorrect assumptions about its functionality, and help them better focus on what Versipass can do to aid them. 


\subsubsection{Memory and Passwords}

Although the difficulty of password policies is often acknowledged, the utter impossibility of these policies is too rarely acknowledged (though Florencio, Herley, and van Oorschot discuss the topic in a recent paper [53]). Humans do not have infinite memory, but there does not appear to be any limit to the number of passwords that they are asked to create and remember. No organization seems willing to say "this is too much".

Although SSO could address the problems with password policies, it is unrealistic to expect that any SSO provider will be used widely enough to address the issue for end users. Accounts are inevitably held with a number of service providers, and we cannot expect that all of those providers will be willing and able to use a single SSO provider. Instead, we must see password managers as the way of the future. Password managers can be controlled by individual users, who can make decisions about their own security. Instead of considering password managers as a flawed solution, the community needs to consider improvements to them.

There are a number of opportunities for password managers to better support users and take on a larger role in the password ecosystem. Password managers provide an excellent lens for users to view their accounts and better understand their web presence and overall security. Existing dedicated password managers (for example, LastPass) do provide some of this functionality, but the browser based managers do not. The internet could also better accommodate password managers. Websites could be constructed with better hooks for password managers, such as XML files providing the password policy directly to the password manager.

Another opportunity for improvement is in the nature of passwords themselves. Password cues allow users to better remember and distinguish their passwords, and are an important part of Versipass's password management strategy. We chose to use graphical passwords because we had previous evidence of the efficacy of the image cues, but text passwords may also be cued. Cues are most effective when there is a clear link between the cue and the cued information [133]. This is easy to achieve with cued graphical passwords, where the image clearly relates to the click points, but harder to accomplish with cued text passwords. Existing cued text passwords link text passwords to stories [14] and images [8], but the additional work that the user must do to link the cue to the password may make them less usable. 


\subsubsection{What is a Password?}

The central concept in Versipass that we think is most likely to be misunderstood is the distinction between the user input and the encoded hashed string that is sent to the website. Which of these is the "password"?

Confusion is likely to occur if we call the user's input clicks the password. A series of clicks is not obviously a password, in the way that most users think of it (as a word). However, we are reluctant to refer to the generated hashed string as the password, because users do not really see that string, and may object to the lack of knowledge about this string.

In our cognitive walkthrough, a discussion arose about locus of control. We were all able to offer anecdotal evidence that users want to have control over their passwords. Users want to pick their passwords, and they want to be able to remember them at will. Using a password manager removes a layer of control over passwords. Users must trust the password manager to consistently generate the same passwords, to save required information, but not save other information.

We speculate that users will feel unhappy about the fact that their input (clicks on a graphical password screen) is not obviously translated into the website password. Both the encoding of the click points and the hashing renders the user's password unpredictable to them. Additionally, having different salts for the same input passwords will mean that the same input will produce different strings for different websites. We suspect that users will feel uncomfortable about having not having control over what their passwords will be, or being able to remember them at will. This is a complex issue: users do not want to have to remember their passwords, but they also don't want to trust someone else to remember their passwords!

We think that one way to handle this issue is to make the salting and hashing less obvious to the user. Websites that follow best practices already salt and hash users' passwords instead of saving them in the clear. However, because this happens invisibly, users are not bothered by it. Perhaps Versipass could achieve a similar level of comfort by hiding the salting and hashing from the user. The design decision is complicated though: we want the user to understand that their input is being transformed differently for each website they use (and the benefits of this), but we do not want them to feel upset by it, or controlled by the password manager. Automating 
and hiding security from users can have awkward consequences because processes are rarely fully hidden, and difficult cases are returned to the users who do not have sufficient information to handle the problem (for example, browsers often ask users to make a decisions about invalid SSL certificates).

Versipass needs to walk a fine line in the information it exposes to the user. The user needs to understand what the password manager is doing, but does not need to be bogged down in understanding the details.

\subsection{Conclusion}

We have developed Versipass, a new approach to login management. Versipass involves the novel combination of two models: the cue model and the category model. Together, these models support the user by providing cues for strong passwords and supporting the safe reuse of each of the passwords across multiple websites. Versipass is designed to support users' existing security behaviour, and also uses graphical passwords to take advantage of users' cognitive abilities.

We suggest that Versipass constitutes a new paradigm for password management by allowing users to create stronger passwords, better remember those passwords, and use a password manager without creating a single location where passwords are stored.

Our prototype implementation and our evaluations have numerous limitations. The current prototype reused code from an earlier project, which impacted both usability and security. However, the prototype allowed us to explore the ideas of the cue and category models, and our evaluations showed where improvements are necessary.

Versipass has brought up several issues involving passwords and password managers. One issue is that the concept of a "password" is becoming more fluid. On the one hand, graphical passwords largely hide what the actual passwords are, but on the other hand, even normal passwords are in fact saved in salted hashed form by any responsible system.

Any knowledge-based authentication must take into account human capabilities. In recent years, it has become the norm for people to have dozens of accounts, and 
we need to acknowledge the impossibility of humans remembering strong passwords for each and every one. Some technology is necessary to bridge this divide. Two approaches are single sign-on and traditional password managers. We suggest that our approach offers new advantages and harnesses users' abilities and current coping practices. 


\section{Chapter 9}

\section{Conclusions}

\subsection{Thesis Summary}

The goal of this thesis was to address the current state of the password problem for end users. Traditional passwords demand much of users, asking them to create, memorize, and manage unique passwords for many accounts. One proposed solution has been graphical passwords. In this thesis, we investigated a number of practical questions about graphical passwords and password use. We explored issues related to the design and memorability of graphical passwords, as well as the strategies that users have to cope with passwords. We were primarily interested in the experiences of end users, and the practical issues that affect them in their everyday lives.

In this work, we explored a variety of issues with passwords for end users. We designed a graphical password system to support the memorability of randomly assigned questions. We used that graphical password system to explore what kind of memory retrieval is best leveraged in the design of graphical password systems, and found that although recognition supports the memorability of passwords, it has associated usability and pragmatic concerns. The results suggest a combination of approaches in the design of graphical passwords to take into account the memorability of passwords, the associated login times, and pragmatic issues such as management of the image cue sets. 
Following on these design questions about graphical passwords, we grew interested in how users were coping with existing text password systems. We conducted interviews with users about how they manage and cope with passwords in their everyday lives. We analyzed the interviews using Grounded Theory, and found that passwords progress through a life cycle, over which they are created, reused, remembered, and forgotten. We concluded that users cope with passwords by rationing their time, memory, and effort to accounts which need more attention. This is a sensible coping strategy that allows users to prioritize more important accounts.

To put the non-expert users into context, we looked at how computer security experts cope with the same password management tasks that confront end users. Experts are more knowledgeable regarding threats to their accounts, and about tools to mitigate those attacks. However, this does not necessarily mean that creating, remembering, and keeping track of large numbers of passwords is easier. Our interviews revealed that experts often use a combination of strategies to manage their passwords, carefully prioritizing high-value accounts and protecting them with secure and high-effort strategies, but using lower-security strategies for accounts of less importance. Even though these strategies seemed to be effective, experts still admitted to having difficulties with password tasks, indicating that all user groups, regardless of expertise, struggle with password management.

The life cycle was developed based on interviews with participants primarily recruited from university communities, and a remaining question was whether the life cycle would reflect the kinds of coping strategies employed by the larger population. We conducted a survey to investigate whether the password life cycle is evident in the coping strategies of a broader population. We found evidence that the survey sample relied on the same kinds of techniques, reusing primary passwords across many accounts, varying passwords slightly for different situations and password policies, and rationing effort across multiple accounts.

Finally, we brought what we learned about increasing the memorability of graphical passwords together with design recommendations from the password life cycle to create Versipass, a login manager to help users better remember and manage their passwords. Versipass allows users to take advantage of the increased memorability of graphical passwords and to more safely reuse passwords across multiple accounts. 


\subsection{Contributions}

This thesis contributes to the literature on usable security in the following ways:

- Randomly assigned passwords are one of the most effective ways to protect users against the threat of guessing attacks, but these passwords can be difficult for users to remember. We improved the PassTiles graphical password system (which was created for previous work) to support the memorability and usability of assigned random passwords.

- Previous work had assumed that recognition memory would best support the memorability of graphical passwords. We conducted three new user studies with 178 participants to investigate this question, and showed that while recognitionbased graphical passwords are memorable, they have poor usability resulting from long login times. We find that systems leveraging cued recall have good memorability and usability, and present the best design choice for graphical passwords.

- We conducted a principled investigation into how users cope with the task of password management. We interviewed 27 users and performed a qualitative analysis of the interview data using Grounded Theory methodology. We concluded that there is a life cycle through which all passwords progress, and discuss ways that the life cycle could contribute to the design of password systems. The life cycle suggests that we need to facilitate safe reuse of passwords.

- We extended our life cycle work and interviewed eleven computer security experts about their coping strategies for password management. We identified particular expert strategies that can be adapted to help non-expert users better manage their password security.

- Our work on the password life cycle led to the emergence of the life cycle theory. We conducted a survey with 348 respondents to explore how the theory and the findings of the interviews extended to a larger population. We found that our survey sample exhibited the same kinds of coping strategies and struggled with the same kinds of problems as the users in our interviews.

- We used the results of our previous studies on memorability and the design implications of the password life cycle to design Versipass, a login manager that 
allows users to take advantage of the benefits of graphical passwords, and also to safely reuse passwords across multiple accounts.

\subsection{Limitations}

Despite presenting practical solutions for end users, there are limitations to the work presented here. Although we attempt to emphasize ecological validity in our work, it can be very difficult to run studies that are both controlled and ecologically valid. In the case of the work on memory retrieval in Chapter 4, we used the MVP framework to run PassTiles on real websites, but it is always difficult to disguise the fact that we are studying password systems when the novel password systems are so obvious to users. As in other studies of this type, positive effects might be due to novelty, or to the participants wishing to please the experimenters. Conversely, there are inherent difficulties in comparing novel graphical password schemes to existing text passwords. Participants have extensive experience with text passwords, including established methods for remembering them. Negative differences seen could be due to participants' lack of experience with graphical passwords, and the fact that they are still establishing mental models for how these passwords work. However, we have taken care to reduce these biases as much as possible in the design of our studies.

In our work on Versipass, we have encountered difficulties when considering how to create an ecologically valid assessment of a password manager. In an ideal situation, we would deploy a working prototype and ask participants to use it on all their accounts. However, there are security, privacy, and ethical concerns with this kind of evaluation. We would like to have instrumented data collection for how people use password managers, but it could be a violation of users' privacy to collect that type of data. As a result, it is difficult to assess Versipass's (or any password manager's) true utility and usability. 


\subsection{Future Research Directions}

The research presented in this thesis suggests a number of future research directions. We believe that PassTiles has the potential to be a usable graphical password system, easy-to-learn and appropriate for different contexts. Although its usability has been explored in the context of the memory retrieval studies, it could be useful to conduct a study whose sole focus is the usability of PassTiles. It would be interesting to compare the usability of PassTiles with that of traditional text passwords, and to explore solutions to some of the obstacles that exist to the real adoption of graphical passwords. We have suggested that PassTiles could be well-suited to mobile devices, and it would be interesting to explore usability in this context.

Another avenue for future work stemming from this thesis would be to improve and further study the Versipass prototype. Improvement of the prototype might involve the development of a browser extension, which would integrate the password manager more strongly with the browser, and allow easier copy/paste access to websites. The design of a new prototype would also create an opportunity to improve the design of the user interface.

More generally, this thesis suggests that better methods of studying and understanding users' real world behaviour are needed. We have stressed the importance of ecological and external validity in our studies, and we believe that in order to create practical solutions to password problems, we cannot make assumptions about how users will behave. For example, the Versipass work raises questions about what kinds of roles password (or login) managers can play in users' password management techniques and coping strategies. Investigating these issues will require great care because of the sensitivity and privacy of users' passwords and account data, but these questions cannot be ignored without limiting the scope of understanding. Two issues arise in these kinds of studies: the need to securely protect users' accounts, and the need for thoughtful and cautious data collection. Participants will need to be assured that their data is secure, and that their privacy will not be compromised. However, we believe that with the development of infrastructure and potential collaboration, these goals are achievable.

We believe that users are handling the difficulties of passwords as well as they are able: users are aware that some accounts have higher priority than others, and users 
make an effort to ration their efforts to create and remember passwords accordingly. Although they reuse passwords, they do so to juggle the demands of many websites. These coping strategies are sensible, and need to be supported in the design of future security products, both for authentication and for other security tasks. 


\section{References}

[1] A. Adams and M. A. Sasse. Users Are Not The Enemy. Communications of the ACM, 42(12):40-46, Dec. 1999.

[2] AgileBits. 1password: Put Passwords In Their Place. https://agilebits. com/onepassword, Accessed 2015.

[3] E. Albrechtsen and J. Hovden. The Information Security Digital Divide Between Information Security Managers and Users. Computers 83 Security, 28(6):476490, Sept. 2009.

[4] Amazon.com, Inc. Amazon Mechanical Turk: Artificial Artificial Intelligence. https://www.mturk. com/mturk/welcome, Accessed 2015.

[5] D. Amitay. Most Common iPhone Passcodes. http://danielamitay.com/ blog/2011/6/13/most-common-iphone-passcodes, June 2011.

[6] J. R. Anderson and G. H. Bower. Recognition and Retrieval Processes in Free Recall. Psychological Review, 79(2):97-123, Mar. 1972.

[7] C. Arthur. Yahoo Voice hack leaks 450,000 passwords http://www. theguardian. com/technology/2012/jul/12/ yahoo-voice-hack-attack-passwords-stolen, July 2012.

[8] Bank of America. SiteKey Security from Bank of America. https: //www. bankof america.com/privacy/online-mobile-banking-privacy/ sitekey.go, Accessed 2015. 
[9] A. Beautement, M. A. Sasse, and M. Wonham. The Compliance Budget: Managing Security Behaviour in Organisations. In Proceedings of the 2009 Workshop on New Security Paradigms, pages 47-58. ACM, 2009.

[10] A. J. Berinsky, G. A. Huber, and G. S. Lenz. Evaluating Online Labor Markets for Experimental Research: Amazon.com's Mechanical Turk. Political Analysis, 20:361-368, Mar. 2012.

[11] R. Biddle, S. Chiasson, and P. C. van Oorschot. Graphical Passwords: Learning from the First Twelve Years. ACM Computing Surveys, 44:19:1-41, 2012.

[12] J.-C. Birget, D. Hong, and N. Memon. Graphical Passwords Based on Robust Discretization. IEEE Transactions on Information Forensics and Security, 1(3):395-399, 2006.

[13] M. Bishop. Proactive Password Checking. In 4th Workshop on Computer Security Incident Handling, pages 1-9, 1992.

[14] J. Blocki, M. Blum, and A. Datta. Naturally Rehearsing Passwords. In ASIACRYPT 2013, pages 361-380. Springer, 2013.

[15] J. Blocki, M. Blum, A. Datta, and S. Vempala. Human Computable Passwords. arXiv:1404.0024v3 [cs.CR], Mar. 2014.

[16] J. Bonneau. The Science of Guessing: Analyzing an Anonymized Corpus of 70 Million Passwords. In Proceedings of the 33rd IEEE Symposium on Security and Privacy, pages 538-552. IEEE, 2012.

[17] J. Bonneau, M. Just, and G. Matthews. What's in a Name? In Financial Cryptography and Data Security, pages 98-113. Springer, 2010.

[18] J. Bonneau, S. Preibusch, and R. Anderson. A Birthday Present Every Eleven Wallets? The Security of Customer-Chosen Banking PINs. In Financial Cryptography and Data Security, pages 25-40. Springer, Aug. 2012.

[19] J. Bonneau and S. Schechter. Towards Reliable Storage of 56-bit Secrets in Human Memory. In Proceedings of the 23rd USENIX Security Symposium, pages 607-623, 2014.

[20] J. Bonneau and E. Shutova. Linguistic Properties of Multi-word Passphrases. In Financial Cryptography and Data Security, pages 1-12. Springer, Oct. 2012. 
[21] D. Boyd. How Parents Normalized Teen Password Sharing. http://www.zephoria.org/thoughts/archives/2012/01/23/ how-parents-normalized-teen-password-sharing.html, Jan. 2012.

[22] R. Breuer. Grammar generation for password recognition. US Patent Office, Patent no. WO2008036879 A2, Mar. 2008.

[23] A. S. Brown, E. Bracken, S. Zoccoli, and K. Douglas. Generating and remembering passwords. Applied Cognitive Psychology, 18(6):641-651, Aug. 2004.

[24] W. E. Burr, D. F. Dodson, and W. T. Polk. NIST Special Publication 800-63: Electronic Authentication Guideline. Technical Report NIST Special Publication 800-63, NIST: National Institute of Standards and Technology, Gaithersburg, USA, Nov. 2006.

[25] W. Cheswick. Rethinking Passwords. Communications of the ACM, 56(2):4044, Feb. 2013.

[26] S. Chiasson, R. Biddle, and P. C. van Oorschot. A Second Look at the Usability of Click-Based Graphical Passwords. In Proceedings of the 3rd Symposium on Usable Privacy and Security. ACM, 2007.

[27] S. Chiasson, C. Deschamps, E. Stobert, M. Hlywa, B. Machado Freitas, A. Forget, N. Wright, G. Chan, and R. Biddle. The MVP Web-based Authentication Framework [Short Paper]. In Financial Cryptography and Data Security, pages 16-24, 2012.

[28] S. Chiasson, A. Forget, R. Biddle, and P. C. van Oorschot. Influencing Users Towards Better Passwords: Persuasive Cued Click-Points. In HCI 2008, pages 121-130. British Computer Society, 2008.

[29] S. Chiasson, A. Forget, R. Biddle, and P. C. van Oorschot. User Interface Design Affects Security: Patterns in Click-Based Graphical Passwords. International Journal of Information Security, 8:387-398, 2009.

[30] S. Chiasson, A. Forget, E. Stobert, P. C. van Oorschot, and R. Biddle. Multiple Password Interference in Text Passwords and Click-Based Graphical Passwords. In Proceedings of the 16th ACM Conference on Computer and Communications Security, pages 500-511. ACM, Nov. 2009. 
[31] S. Chiasson, J. Srinivasan, R. Biddle, and P. C. van Oorschot. Centered Discretization with Application to Graphical Passwords. In Usability, Psychology, and Security. USENIX Association, Apr. 2008.

[32] S. Chiasson, E. Stobert, A. Forget, R. Biddle, and P. C. van Oorschot. Persuasive Cued Click-Points: Design, Implementation, and Evaluation of a Knowledge-Based Authentication Mechanism. IEEE Transactions on Dependable and Secure Computing, 9(2):222-235, Oct. 2011.

[33] S. Chiasson, P. C. van Oorschot, and R. Biddle. A Usability Study and Critique of Two Password Managers. In Proceedings of the 15th USENIX Security Symposium. USENIX Association, 2006.

[34] S. Chiasson, P. C. van Oorschot, and R. Biddle. Graphical Password Authentication Using Cued Click Points. In European Symposium On Research In Computer Security, pages 359-374. Springer, Sept. 2007.

[35] L. Coventry. Usable Biometrics. In L. F. Cranor and S. L. Garfinkel, editors, Security and Usability: Designing Secure Systems That People Can Use, pages 175-197. O'Reilly Media, 2005.

[36] F. I. M. Craik and R. S. Lockhart. Levels of Processing: A Framework for Memory Research. Journal of Verbal Learning and Verbal Behavior, 11:671684, 1972.

[37] H. Crawford and J. Aycock. Kwyjibo: automatic domain name generation. Software: Practice and Experience, 38(14):1561-1567, Nov. 2008.

[38] CrowdFlower, Inc. CrowdFlower|People-powered Data Enrichment Platform. http://www. crowdflower. com, Accessed 2015.

[39] N. Cubrilovic. RockYou Hack: From Bad to Worse. http://techcrunch.com/ 2009/12/14/rockyou-hack-security-myspace-facebook-passwords/, Dec. 2009 .

[40] A. Das, J. Bonneau, M. Caesar, N. Borisov, and X. Wang. The Tangled Web of Password Reuse. In Network and Distributed System Security Symposium, San Diego, USA, Feb. 2014. The Internet Society. 
[41] D. Davis, F. Monrose, and M. K. Reiter. On User Choice in Graphical Password Schemes. In Proceedings of the 13th USENIX Security Symposium, pages 151164. USENIX Association, 2004.

[42] A. De Angeli, L. Coventry, G. Johnson, and K. Renaud. Is a picture really worth a thousand words? Exploring the feasibility of graphical authentication systems. International Journal of Human-Computer Studies, 63(1-2):128-152, July 2005.

[43] S. Designer. John the Ripper Password Cracker. http://www .openwall.com/ john/, Accessed 2011.

[44] R. Dhamija and A. Perrig. Déjà Vu: A User Study Using Images for Authentication. In Proceedings of the 13th USENIX Security Symposium. USENIX Association, 2000.

[45] P. Dunphy and J. Yan. Do Background Images Improve "Draw a Secret" Graphical Passwords? In Proceedings of the 14th Conference on Computer and Communications Security, pages 36-47. ACM, Oct. 2007.

[46] eBay. eBay Inc. To Ask eBay Users To Change Passwords. http://www.ebayinc.com/in_the_news/story/ ebay-inc-ask-ebay-users-change-passwords, May 2014.

[47] S. Egelman, J. Bonneau, S. Chiasson, D. Dittrich, and S. Schechter. It's Not Stealing If You Need It: A Panel on the Ethics of Performing Research Using Public Data of Illicit Origin. In Financial Cryptography and Data Security, pages 124-132. Springer, 2012.

[48] S. Egelman, A. J. B. Brush, and K. M. Inkpen. Family accounts. In Proceedings of the Conference on Computer Supported Cooperative Work, pages 669-678. ACM, 2008.

[49] S. Egelman, A. Sotirakopoulos, I. Muslukhov, K. Beznosov, and C. Herley. Does My Password Go Up to Eleven?: The Impact of Password Meters on Password Selection. In Proceedings of the SIGCHI Conference on Human Factors in Computing Systems, pages 2379-2388. ACM, 2013. 
[50] D. Florencio and C. Herley. A Large-Scale Study of Web Password Habits. In Proceedings of the International World Wide Web Conference, pages 657-666. IW3C2, 2007.

[51] D. Florencio and C. Herley. Where Do Security Policies Come From? In Proceedings of the 6th Symposium on Usable Privacy and Security. ACM, 2010.

[52] D. Florencio, C. Herley, and P. C. van Oorschot. An Administrator's Guide to Internet Password Research. In Proceedings of the 28th USENIX Large Installation System Administration Conference, pages 35-52. USENIX Association, 2014.

[53] D. Florencio, C. Herley, and P. C. van Oorschot. Password Portfolios and the Finite-Effort User: Sustainably Managing Large Numbers of Accounts. In Proceedings of the 23rd USENIX Security Symposium, pages 575-590. USENIX Association, Aug. 2014.

[54] A. Forget, S. Chiasson, P. C. van Oorschot, and R. Biddle. Improving Text Passwords Through Persuasion. In Proceedings of the 4th Symposium on Usable Privacy and Security, pages 1-12. ACM, 2008.

[55] R. Ganesan, C. Davies, and B. Atlantic. A New Attack on Random Pronounceable Password Generators. In Proceedings of the 17th National Computer Security Conference. NIST, 1994.

[56] M. Gasser. A Random Word Generator for Pronounceable Passwords. Technical Report ESD-TR-75-97, The MITRE Corporation, Nov. 1975.

[57] S. Gaw and E. W. Felten. Password Management Strategies for Online Accounts. In Proceedings of the 2nd Symposium on Usable Privacy and Security, pages 44-55. ACM, 2006.

[58] V. V. Gouveia and M. Ros. Hofstede and Schwartz's Models for Classifying Individualism at the Cultural Level: Their Relation to Macro-Social and MacroEconomic Variables. Psicothema, 12(Suplemento):25-33, Dec. 2000.

[59] B. Grawemeyer and H. Johnson. Using and Managing Multiple Passwords: a Week to a View. Interacting with Computers, 23(3):256-267, May 2011. 
[60] GrIDsure. Pattern Based Authentication. http://gridsure-security.co.uk, Accessed 2015.

[61] Hacker News. I'm the Chrome browser security tech lead, so it might help if I explain our reasoning here. https://news.ycombinator.com/item?id= 6166731, 2013.

[62] F. Haist, A. P. Shimamura, and L. R. Squire. On the Relationship Between Recall and Recognition Memory. Journal of Experimental Psychology: Learning, Memory, and Cognition, 18:691-702, 1992.

[63] E. Hayashi and J. Hong. A diary study of password usage in daily life. In Proceedings of the International Conference on Human Factors in Computing Systems, pages 2627-2630. ACM, May 2011.

[64] C. Herley. So Long, and No Thanks for the Externalities: The Rational Rejection of Security Advice by Users. In Proceedings of the 2009 Workshop on New Security Paradigms, pages 133-144. ACM, 2009.

[65] C. Herley and P. C. van Oorschot. A Research Agenda Acknowledging the Persistence of Passwords. IEEE Security $\mathscr{G}$ Privacy, 10(1):28-36, 2012.

[66] G. Hofstede. National Cultures in Four Dimensions: A Research-Based Theory of Cultural Differences Among Nations. International Studies of Management E Organization, 13(1/2):46-74, Oct. 2005.

[67] H. L. Hollingworth. Characteristic Differences between Recall and Recognition. American Journal of Psychology, 24(4):532-544, Oct. 1913.

[68] B. Ives, K. R. Walsh, and H. Schneider. The Domino Effect of Password Reuse. Communications of the ACM, 47(4):75-78, Apr. 2004.

[69] L. L. Jacoby and W. H. Bartz. Rehearsal and Transfer to LTM. Journal of Verbal Learning and Verbal Behavior, 11(5):561-565, 1972.

[70] I. Jermyn, A. Mayer, F. Monrose, M. K. Reiter, and A. D. Rubin. The Design and Analysis of Graphical Passwords. In Proceedings of the 8th USENIX Security Symposium. USENIX Association, 1999.

[71] M. Just. Designing and Evaluating Challenge-Question Systems. IEEE Security E) Privacy, 2(5):32-39, 2004. 
[72] A. Karole, N. Saxena, and N. Christin. A Comparative Usability Evaluation of Traditional Password Managers. In Information Security and Cryptology, pages 233-251. Springer, 2011.

[73] J. Kaye. Self-Reported Password Sharing Strategies. In Proceedings of the SIGCHI Conference on Human Factors in Computing Systems, pages 26192622. ACM, 2011.

[74] M. Keith, B. Shao, and P. Steinbart. A Behavioral Analysis of Passphrase Design and Effectiveness. Journal of the Association for Information Systems, 10(2):63-89, Feb. 2009.

[75] M. Keith, B. Shao, and P. J. Steinbart. The Usability of Passphrases for Authentication: an Empirical Field Study. International Journal of Human-Computer Studies, 65(1):17-28, 2007.

[76] P. G. Kelley. Conducting Usable Privacy \& Security Studies with Amazon's Mechanical Turk. In Usable Security Experiment Reports Workshop, 2010.

[77] P. G. Kelley, S. Komanduri, M. M. Mazurek, R. Shay, T. Vidas, L. Bauer, N. Christin, and L. F. Cranor. The impact of length and mathematical operators on the usability and security of one-time PINs. In Financial Cryptography and Data Security, pages 34-51. Springer, 2013.

[78] A. Kittur, E. H. Chi, and B. Suh. Crowdsourcing user studies with Mechanical Turk. In Proceedings of the International Conference on Human Factors in Computing Systems, pages 453-456. ACM, 2008.

[79] S. Komanduri, R. Shay, L. F. Cranor, C. Herley, and S. Schechter. Telepathwords: Preventing weak passwords by reading users' minds. In Proceedings of the 23rd USENIX Security Symposium, pages 591-606. USENIX Association, 2014.

[80] S. Komanduri, R. Shay, P. G. Kelley, M. M. Mazurek, L. Bauer, N. Christin, L. F. Cranor, and S. Egelman. Of Passwords and People: Measuring the Effect of Password-Composition Policies. In Proceedings of the International Conference on Human Factors in Computing Systems, pages 2595-2604. ACM, 2011.

[81] M. Kotadia. Gates predicts death of the password. http://news.cnet.com/ 2100-1029-5164733.html, Feb. 2004. 
[82] C. Kuo, S. Romanosky, and L. F. Cranor. Human Selection of Mnemonic Phrase-based Passwords. In Proceedings of the 2nd Symposium on Usable Privacy and Security, pages 67-78. ACM, July 2006.

[83] LastPass. LastPass: Simplify Your Life. http://lastpass.com, Accessed 2015.

[84] S. Lau, S. Siena, A. Pandey, S. Sosothikul, L. F. Cranor, B. Ur, and R. Shay. Exploring the Usability of Pronounceable Passwords (Poster). In Proceedings of the 10th Symposium on Usable Privacy and Security, 2014.

[85] M. D. Leonhard and V. N. Venkatakrishnan. A Comparative Study of Three Random Password Generators. In International Conference on Electro/Information Technology. IEEE, 2007.

[86] Z. Li, W. He, D. Akhawe, and D. Song. The Emperor's New Password Manager: Security Analysis of Web-Based Password Managers. In Proceedings of the 23rd USENIX Security Symposium, pages 465-479. USENIX Association, Aug. 2014.

[87] M. E. Locasto, M. Massimi, and P. J. DePasquale. Security and Privacy Considerations in Digital Death. In Proceedings of the 2011 Workshop on New Security Paradigms, pages 1-10. ACM, 2011.

[88] M. Mannan and P. C. van Oorschot. Security and Usability: The Gap in RealWorld Online Banking. In Proceedings of the 2007 Workshop on New Security Paradigms, pages 1-14. ACM, July 2008.

[89] M. M. Mazurek, S. Komanduri, T. Vidas, L. Bauer, N. Christin, L. F. Cranor, P. G. Kelley, R. Shay, and B. Ur. Measuring password guessability for an entire university. In Proceedings of the ACM Conference on Computer and Communications Security, pages 173-186. ACM, Nov. 2013.

[90] A. J. Menezes, S. Vanstone, and P. C. van Oorschot. Handbook of Applied Cryptography. CRC Press, Inc., Boca Raton, USA, 1996.

[91] M. Z. Mintzer and J. G. Snodgrass. The Picture Superiority Effect: Support for the Distinctiveness Model. The American Journal of Psychology, 112(1):113146, 1999.

[92] R. Morris and K. Thompson. Password Security: A Case History. Communications of the ACM, 22(11):594-597, Nov. 1979. 
[93] my1login. myllogin: Take Control of Your Business Passwords. https://www . my1login. com/content/index.php, Accessed 2015.

[94] D. L. Nelson and V. S. Reed. On the Nature of Pictorial Encoding: A Levelsof-Processing Analysis. Journal of Experimental Psychology: Human Learning and Memory, 2(1):49-57, 1976.

[95] D. L. Nelson, V. S. Reed, and C. L. McEvoy. Learning to Order Pictures and Words: A Model of Sensory and Semantic Encoding. Journal of Experimental Psychology: Human Learning and Memory, 3(5):485-497, Sept. 1977.

[96] D. L. Nelson, V. S. Reed, and J. R. Walling. Pictorial Superiority Effect. Journal of Experimental Psychology: Human Learning and Memory, 2(5):523-528, 1976.

[97] D. A. Norman. When Security Gets in the Way. ACM SIGCSE Bulletin, 16(6):60-63, Nov. 2009.

[98] G. Notoatmodjo. Exploring the 'Weakest Link': A Study of Personal Password Security. Master's thesis, The University of Auckland, New Zealand, Nov. 2007.

[99] W. Odom, J. Zimmerman, and J. Forlizzi. Designing for Dynamic Family Structures. In Proceedings of the 8th Conference on Designing Interactive Systems, pages 368-371. ACM, 2010.

[100] A. Paivio. Imagery and Verbal Processes. Holt, Rinehart, and Winston, 1971.

[101] A. Paivio, T. B. Rogers, and P. C. Smythe. Why Are Pictures Easier to Recall Than Words? Psychonomic Science, 11(4):137-138, 1968.

[102] A. Pashalidis and C. J. Mitchell. A Taxonomy of Single Sign-On Systems. In Information Security and Privacy, pages 249-264. Springer, June 2003.

[103] Password Box. Password Box: The Most Trusted Identity Manager with over 14 Million Downloads. https://www. passwordbox.com/, Accessed 2015.

[104] Peter Pauper Press. The Personal Internet Address 83 Password Log Book (Organizer). Peter Pauper Press, Accessed 2014.

[105] Real User Corporation. The Science Behind Passfaces. http://www. passfaces.com/published/The\%20Science\%20Behind\%20Passfaces.pdf, June 2004 . 
[106] D. Reichl. KeePass Password Safe. http://keepass .info/, Accessed 2015.

[107] K. Renaud and M. Just. Pictures or Questions?: Examining User Responses to Association-Based Authentication. In Proceedings of the 24th BCS Conference on Human-Computer Interaction, pages 98-107. British Computer Society, 2010.

[108] M. Richtel. Teenagers Sharing Passwords as Show of Affection. http://www.nytimes.com/2012/01/18/us/ teenagers-sharing-passwords-as-show-of-affection.html, Jan. 2012.

[109] B. L. Riddle, M. S. Miron, and J. A. Semo. Passwords in Use in a University Timesharing Environment. Computers \&3 Security, 8(7):569-579, 1989.

[110] B. Ross, C. Jackson, N. Miyake, D. Boneh, and J. C. Mitchell. Stronger Password Authentication Using Browser Extensions. In Proceedings of the 14th USENIX Security Symposium. USENIX Association, Aug. 2005.

[111] S. Schechter, A. J. B. Brush, and S. Egelman. It's No Secret. Measuring the Security and Reliability of Authentication via "Secret" Questions. Proceedings of the 30th IEEE Symposium on Security and Privacy, pages 375-390, 2009.

[112] S. Schechter, C. Herley, and M. Mitzenmacher. Popularity Is Everything: A New Approach to Protecting Passwords From Statistical-Guessing Attacks. In Proceedings of the 5th USENIX Workshop on Hot Topics in Security. USENIX Association, 2010.

[113] Schmitz, Carsten. Limesurvey - The free and open source survey software tool! https://www.limesurvey.org/, Accessed 2015.

[114] B. Schneier. Write Down Your Password. http://www.schneier.com/blog/ archives/2005/06/write_down_your.html, June 2005.

[115] B. Schneier. Choosing a Secure Password. http://boingboing. net/2014/02/ 25/choosing-a-secure-password.html, Feb. 2014.

[116] B. Schwartz, A. Benjamin, and R. A. Bjork. The Inferential and Experiential Bases of Metamemory. Current Directions in Psychological Science, 6(5):132137, 1997. 
[117] R. Shay, P. G. Kelley, S. Komanduri, M. M. Mazurek, B. Ur, T. Vidas, L. Bauer, N. Christin, and L. F. Cranor. Correct Horse Battery Staple: Exploring the Usability of System-Assigned Passphrases. In Proceedings of the 8th Symposium on Usable Privacy and Security. ACM, July 2012.

[118] R. Shay, S. Komanduri, P. G. Kelley, P. G. Leon, M. M. Mazurek, L. Bauer, N. Christin, and L. F. Cranor. Encountering Stronger Password Requirements: User Attitudes and Behaviors. In Proceedings of the 6th Symposium on Usable Privacy and Security. ACM, June 2010.

[119] Siber Systems Inc. Roboform: Make Your Life Easier with the Top Rated Password Manager. http://www.roboform.com, Accessed 2015.

[120] D. Silver, S. Jana, E. Chen, C. Jackson, and D. Boneh. Password Managers: Attacks and Defenses. In Proceedings of the 23rd USENIX Security Symposium, pages 449-464. USENIX Association, Aug. 2014.

[121] S. Singh, A. Cabraal, C. Demosthenous, G. Astbrink, and M. Furlong. Password Sharing: Implications for Security Design Based on Social Practice. In Proceedings of the International Conference on Human Factors in Computing Systems, pages 895-904. ACM, 2007.

[122] E. H. Spafford. Observing Reusable Password Choices. In Proceedings of the 3rd UNIX Security Symposium. USENIX Association, Sept. 1992.

[123] M. Steves, D. Chisnell, M. A. Sasse, K. Krol, M. Theofanos, and H. Wald. Report: Authentication Diary Study. Technical report, National Institute of Standards and Technology, Information Technology Laboratory, Gaithersburg, MD, Feb. 2014.

[124] E. Stobert. Memorability of Assigned Random Graphical Passwords. Master's thesis, Carleton University, Department of Psychology, Aug. 2011.

[125] E. Stobert and R. Biddle. Authentication in the Home. In Workshop on Home Usable Privacy and Security, 2013.

[126] E. Stobert and R. Biddle. Memory retrieval and graphical passwords. In Proceedings of the 9th Symposium on Usable Privacy and Security, number 15. ACM, 2013. 
[127] E. Stobert, S. Chiasson, and R. Biddle. User-Choice Patterns in PassTiles Graphical Passwords (Poster). In Annual Computer Security Application Conference, Dec. 2011.

[128] A. Strauss and J. Corbin. Basics of Qualitative Research: Techniques and Procedures for Developing Grounded Theory. SAGE Publications, Thousand Oaks, California, 2nd edition, 1998.

[129] S.-T. Sun, Y. Boshmaf, K. Hawkey, and K. Beznosov. A Billion Keys, but Few Locks. In Proceedings of the 2010 Workshop on New Security Paradigms, pages 61-72. ACM, 2010.

[130] S.-T. Sun, E. Pospisil, I. Muslukhov, N. Dindar, K. Hawkey, and K. Beznosov. What Makes Users Refuse Web Single Sign-On?: An Empirical Investigation of OpenID. In Proceedings of the rth Symposium on Usable Privacy and Security, number 4. ACM, 2011.

[131] Tecca. Over 55,000 Twitter passwords exposed, posted online. http://news. yahoo.com/blogs/technology-blog/ over-55-000-twitter-passwords-exposed-posted-online-165625320. html, May 2012.

[132] E. Tulving. When Is Recall Higher Than Recognition? Psychonomic Science, 10(2):53-54, 1968.

[133] E. Tulving and D. Thomson. Encoding Specificity and Retrieval Processes in Episodic Memory. Psychological Review, 80(5):352-373, 1973.

[134] E. Tulving and M. J. Watkins. Continuity between Recall and Recognition. American Journal of Psychology, 86(4):739-748, 1973.

[135] B. Ur, P. G. Kelley, S. Komanduri, J. Lee, M. Maass, M. M. Mazurek, T. Passaro, R. Shay, T. Vidas, L. Bauer, N. Christin, and L. F. Cranor. How Does Your Password Measure Up? The Effect of Strength Meters on Password Creation. In Proceedings of the 21st USENIX Security Symposium, pages 65-80. USENIX Association, Aug. 2012.

[136] P. C. van Oorschot and J. Thorpe. On Predictive Models and User-Drawn Graphical Passwords. ACM Transactions on Information and System Security, 10(4), Jan. 2008. 
[137] P. C. van Oorschot and J. Thorpe. Exploiting Predictability in Click-based Graphical Passwords. Journal of Computer Security, 19(4):669-702, 2011.

[138] E. von Zezschwitz, A. De Luca, and H. Hussmann. Survival of the Shortest: A Retrospective Analysis of Influencing Factors on Password Composition. In Proceedings of the 14th International Conference on Human-Computer Interaction, pages 460-467. Springer, July 2013.

[139] M. J. Watkins. When Is Recall Spectacularly Higher Than Recognition? Journal of Experimental Psychology, 102(1):161-163, 1974.

[140] M. J. Watkins and J. M. Gardiner. An Appreciation of Generate-Recognize Theory of Recall. Journal of Verbal Learning and Verbal Behavior, 18(6):687704, Dec. 1979.

[141] M. Weir, S. Aggarwal, M. Collins, and H. Stern. Testing Metrics for Password Creation Policies by Attacking Large Sets of Revealed Passwords. In Proceedings of the 17th ACM Conference on Computer and Communications Security, pages 162-175. ACM, Oct. 2010.

[142] M. S. Weldon and H. L. Roediger, III. Altering Retrieval Demands Reverses the Picture Superiority Effect. Memory 83 Cognition, 15(4):269-280, Jan. 1987.

[143] C. Wharton, J. Rieman, C. Lewis, and P. Polson. The Cognitive Walkthrough Method: A Practitioner's Guide. In J. Nielsen and R. L. Mack, editors, Usability Inspection Methods, pages 105-140. John Wiley \& Sons, Inc., New York, USA, 1994.

[144] A. White, K. Shaw, F. Monrose, and E. Moreton. Isn't that Fantabulous: Security, Linguistic and Usability Challenges of Pronounceable Tokens. In Proceedings of the 2014 Workshop on New Security Paradigms, pages 25-38. ACM, 2014.

[145] L. Whitney. Millions of LinkedIn passwords reportedly leaked online. http://www.cnet.com/news/ millions-of-linkedin-passwords-reportedly-leaked-online/, June 2012 . 
[146] A. Whitten and J. D. Tygar. Why Johnny Can't Encrypt: A Usability Evaluation of PGP 5.0. In Proceedings of the 8th USENIX Security Symposium, pages 169-183. USENIX Association, 1999.

[147] S. Wiedenbeck, J. Waters, J.-C. Birget, A. Brodskiy, and N. Memon. PassPoints: Design and Longitudinal Evaluation of a Graphical Password System. International Journal of Human-Computer Studies, 63(1-2):102-127, July 2005.

[148] J. Yan, A. F. Blackwell, R. Anderson, and A. Grant. Password Memorability and Security: Empirical Results. IEEE Security $\mathscr{6}$ Privacy, 2(5):25-31, Sept. 2004.

[149] B. B. Zhu, D. Wei, M. Yang, and J. Yan. Security Implications of Password Discretization for Click-Based Graphical Passwords. In Proceedings of the International World Wide Web Conference. IW3C2, May 2013.

[150] B. B. Zhu, J. Yan, D. Wei, and M. Yang. Security Analyses of Click-based Graphical Passwords via Image Point Memorability. In Proceedings of the 21st Conference on Computer and Communications Security, pages 1217-1231. ACM, 2014.

[151] M. Zviran and W. J. Haga. User Authentication by Cognitive Passwords: an Empirical Assessment. In Proceedings of the 5th Jerusalem Conference on Information Technology, 1990.

[152] M. Zviran and W. J. Haga. Password Security: An Empirical Study. Journal of Management Information Systems, 15(4):161-185, 1999. 


\section{Appendix A \\ Results of MA Memory Studies}

\section{A.1 Introduction}

In this appendix, we include the results of two studies investigating what type of memory retrieval is best leveraged in the design of graphical passwords. These studies were conducted for my MA (Psychology, 2011), and were published along with the studies in Chapter 4. The background to these studies is presented in Chapter 4, and we include the details of the results here.

\section{A.2 Results}

\section{A.2.1 Study 1}

Study 1 was conducted in a hybrid format, where Session 1 was conducted in person, Session 2 took place online, and Session 3 was in person. The goal of the study was to investigate how the different types of memory retrieval affected the usability and memorability of assigned graphical passwords.

Study 1 had 81 participants ( 45 female), recruited from both the university and the local community. Participants ranged in age from 18 to 62 , with a median age of 
24. 53 participants were students from a broad range of degree programs and levels, and the remaining participants were occupied in a broad variety of fields. None of the students were studying or working in the field of computer security. Fifteen participants reported having previously used a graphical password system, and all were referring to systems where the website provided an image for labelling as part of the authentication process. 8 participants reported having previously participated in a website study, and 5 participants reported previous participation in a password study.

We had three hypotheses, each applying to one of the dependent variables studied:

H1(a): Memory time would be significantly shorter for Assigned Text than for the three graphical password conditions.

H1(b): There would be significant differences in memory time among the three graphical password conditions.

H1(c): Memory time would be significantly longer for Chosen Text than for any of the assigned password conditions.

H2(a): There would be significantly more password resets in Assigned Text than in any of the three graphical password conditions.

H2(b): There would be significant differences in the number of password resets among three graphical password conditions.

H2(c): There would be significantly fewer password resets in Chosen Text than in any of the assigned password conditions.

H3: There would be significant differences in login times among the five study conditions.

\section{Hypothesis 1: Memory Time}

Table A.1 shows descriptive statistics for the memory time variable. To aggregate across the three websites used in the study, we took the mean of each participant's memory time for each website. Median memory time ranged between 164.14 hours in 


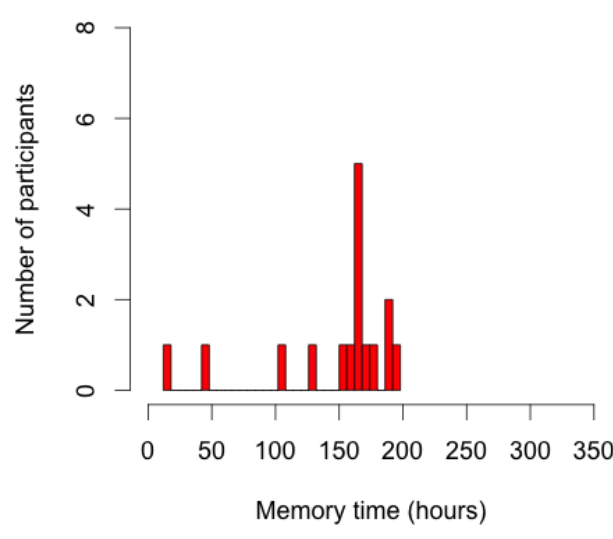

(a) BPT

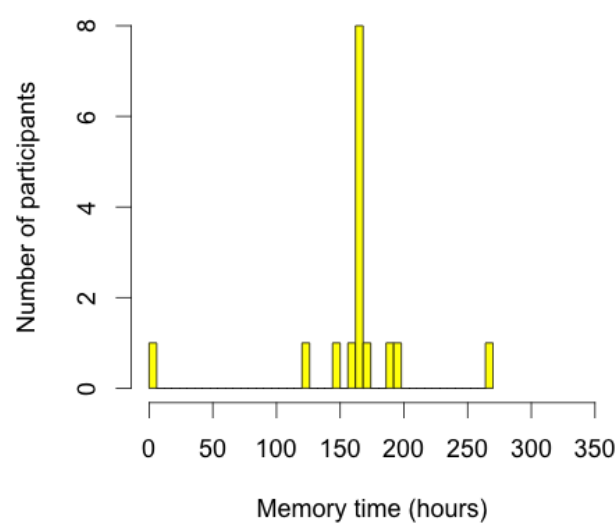

(c) OPT

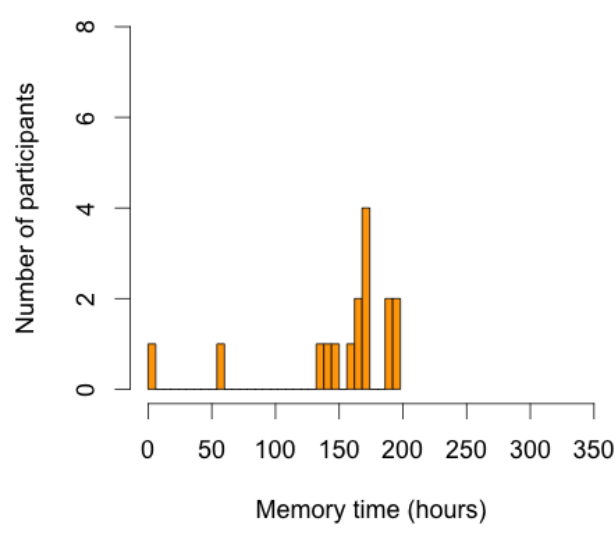

(b) IPT

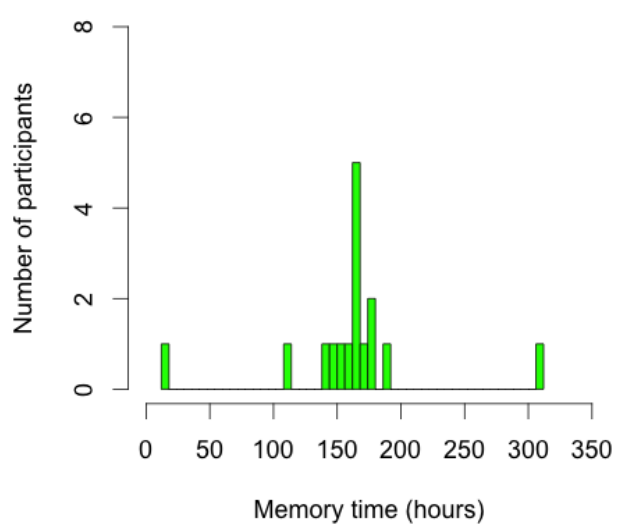

(d) AST

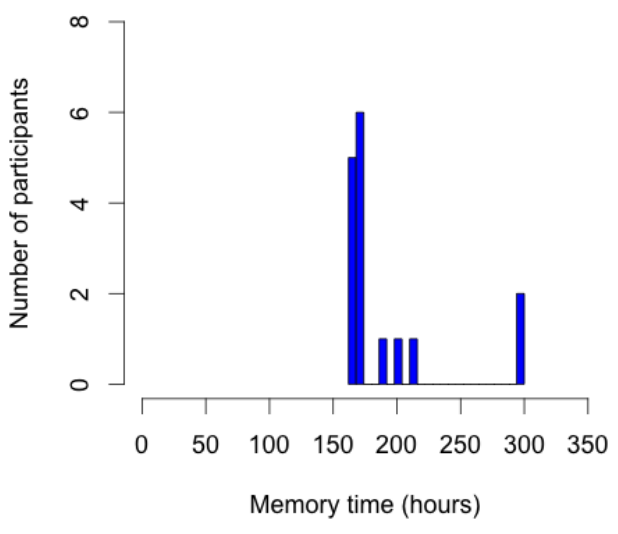

(e) $\mathrm{CHT}$

Figure A.1: Distributions of memory time (in hours) by number of participants for each study condition (Study 1). 
Table A.1: Memory time statistics (hours) (Study 1).

\begin{tabular}{|l|r|r|r|r|r|}
\hline & Mean & SD & Median & Skewness & Kurtosis \\
\hline BPT & 147.00 & 50.57 & 164.14 & -1.77 & 2.57 \\
IPT & 150.76 & 51.90 & 167.63 & -2.16 & 4.61 \\
OPT & 160.71 & 51.96 & 166.90 & -1.63 & 7.05 \\
AST & 160.67 & 56.80 & 166.57 & -0.02 & 5.60 \\
CHT & 190.62 & 44.08 & 168.73 & 2.09 & 3.35 \\
\hline
\end{tabular}

Blank PassTiles and 168.73 hours in Chosen Text. The total duration of the study was 7 days (approximately 168 hours), so this result shows that most participants were able to remember their passwords for the entire study. We excluded one participant from the analysis because they had to leave town during the study. Since most participants returned for the second session after exactly 7 days (a few participants returned after 8 or 9 days due to scheduling constraints), the memory time was limited by this aspect of the study design. We were surprised to find that all participants had been able to remember their passwords so long.

Histograms of the distributions of memory time (in hours) (Figure A.1) for each of the five conditions suggested that the distributions are approximately normal. Some conditions were leptokurtotic, but the measures of skewness and kurtosis (Table A.1) indicated that this was unlikely to affect the results and we conducted further analysis using ANOVA and $t$-tests. We follow this procedure in all tests we report here, using parametric tests (ANOVA and $t$-tests) where appropriate, and otherwise apply ordinal tests (Kruskal-Wallis and Wilcoxon (Mann-Whitney) tests).

Table A.2: $t$-tests of memory time (Study 1$)$.

\begin{tabular}{|l|r|r|r|}
\hline & $t$ & df & $p$ \\
\hline AST vs. BPT & 0.72 & 30 & 0.761 \\
AST vs. IPT & 0.52 & 30 & 0.695 \\
AST vs. OPT & -0.00 & 30 & 0.499 \\
\hline
\end{tabular}

H1(a): We conducted three one-sided $t$-tests (Table A.2), each comparing a graphical password condition to Assigned Text, and found no significant differences in memory time between Assigned Text and any of the graphical password conditions. This provided no evidence that participants were able to remember graphical passwords longer than assigned text passwords. Since these comparisons were hypothesized $a$ priori, we did not correct for multiple tests. 
H1(b): A one-way ANOVA of memory time showed no significant differences in memory time among the three graphical password conditions $(F(2,45)=0.30, p=$ $0.740)$.

H1(c): We conducted a set of four a priori $t$-tests comparing memory time for Chosen Text with memory time for each of the other study conditions. A significant difference in memory time was seen between Chosen Text and Blank PassTiles $(t(29)=2.60, p=$ $0.007)$, between Chosen Text and Image PassTiles $(t(29)=2.34, p=0.013)$, and between Chosen Text and Object PassTiles $(t(29)=1.76, p=0.045)$. We suspect that this result reflects a chance occurrence where more Chosen Text participants returned after 7 days, artificially creating a difference in memory times.

\section{Hypothesis 2: Resets}

Table A.3: Password reset statistics (Study 1).

\begin{tabular}{|l|r|r|r|r|r|}
\hline & Mean & SD & Median & Skewness & Kurtosis \\
\hline BPT & 0.88 & 1.67 & 0 & 1.70 & 1.61 \\
IPT & 0.75 & 1.73 & 0 & 2.55 & 5.98 \\
OPT & 0.12 & 0.50 & 0 & 4.00 & 16.00 \\
AST & 0.62 & 1.15 & 0 & 2.07 & 4.26 \\
CHT & 0.00 & 0.00 & 0 & 0.00 & -3.66 \\
\hline
\end{tabular}

As a measure of the memorability of the password systems, we recorded the number of password resets. For each participant, we took the total number of resets on all websites. Participants were free to reset their passwords at any time during the at-home sessions of the study.

The median number of resets was 0 in all conditions, indicating that most participants never reset any of their passwords. Table A.3 shows descriptive statistics for password resets. Figure A.2 shows the distributions of password resets for each condition. As seen in the histograms, the distributions of resets were skewed and kurtotic, making parametric tests unsuitable. Wilcoxon tests were conducted in place of $t$-tests, and Kruskal-Wallis tests were used in place of one-way ANOVAs.

H2(a): Wilcoxon tests showed no significant difference in the number of password resets between Assigned Text and either Image PassTiles or Blank PassTiles. However, participants reset their passwords significantly more often in Assigned Text than 


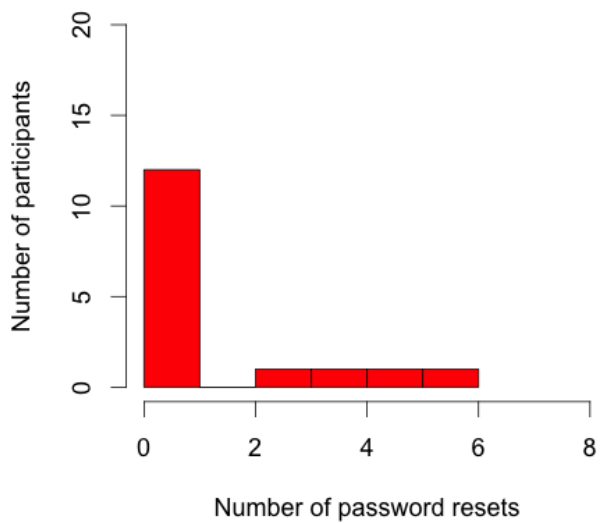

(a) $\mathrm{BPT}$

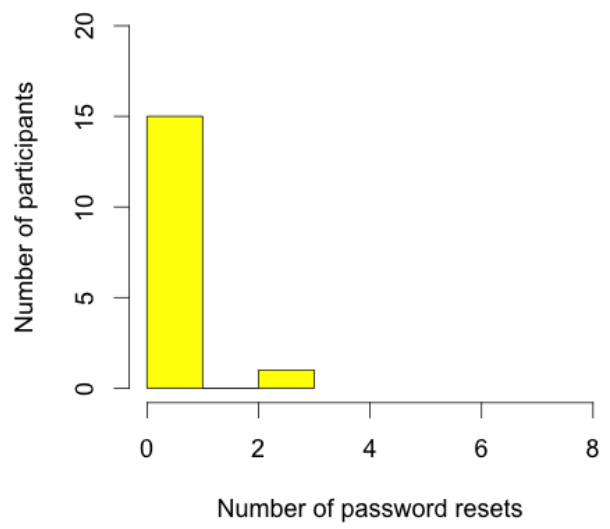

(c) OPT

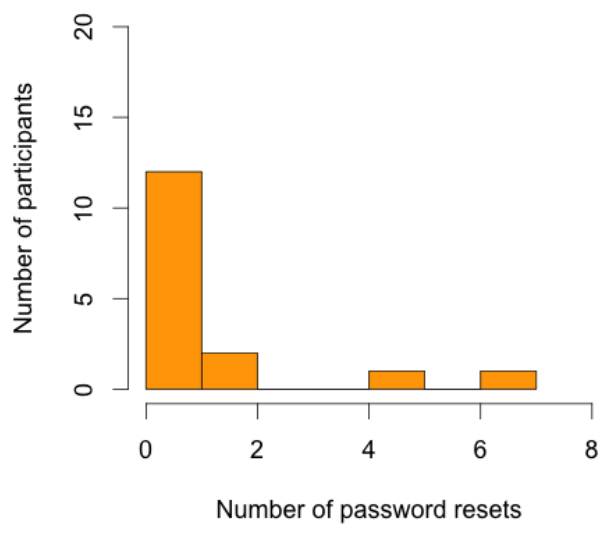

(b) IPT

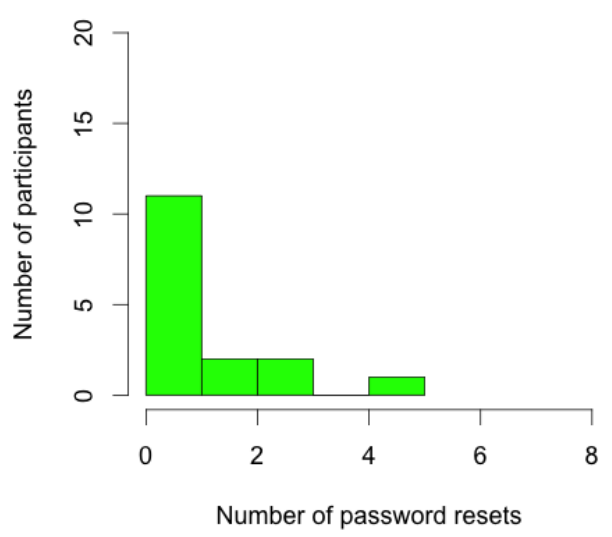

(d) AST

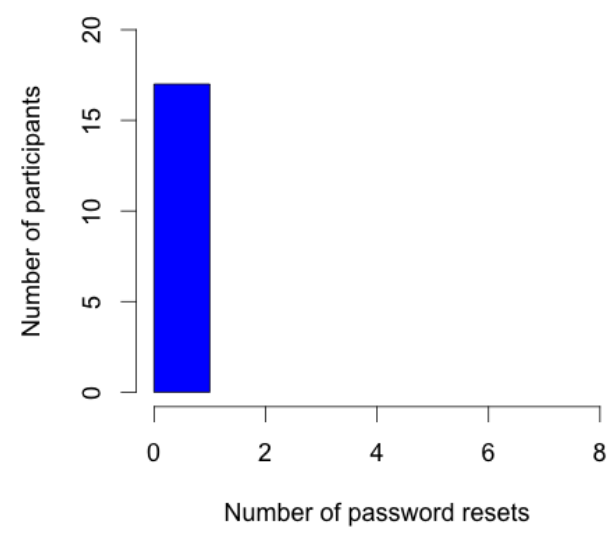

(e) $\mathrm{CHT}$

Figure A.2: Distributions of resets by number of participants for each study condition (Study 1). 
in Object PassTiles $(U=159.50, p=0.043)$. Again, since these comparisons were hypothesized a priori, we did not correct for multiple tests.

H2(b): A Kruskal-Wallis test showed no significant difference in the number of password resets among the three graphical password conditions $\left(\chi^{2}(2)=2.54, p=0.282\right)$, providing no evidence that participants reset their passwords differently in any of the PassTiles conditions.

H2(c): We conducted four a priori one-way Wilcoxon tests, and found a significant difference in the number of password resets between Chosen Text and each of Blank PassTiles $(U=102.00, p=0.017)$, Image PassTiles $(U=102.00, p=0.017)$, and Assigned Text $(U=93.50, p=0.008)$. This indicated that in all conditions except Object PassTiles, participants reset their passwords more often in the assigned text condition.

\section{Hypothesis 3: Login Times}

Table A.4: Login time statistics (seconds) (Study 1).

\begin{tabular}{|l|r|r|r|r|r|}
\hline & Mean & SD & Median & Skewness & Kurtosis \\
\hline BPT & 15.74 & 10.48 & 13.38 & 3.00 & 10.19 \\
IPT & 12.65 & 6.14 & 9.33 & 1.38 & 1.52 \\
OPT & 34.61 & 21.79 & 24.25 & 1.74 & 2.03 \\
AST & 9.06 & 3.92 & 8.28 & 2.12 & 5.76 \\
CHT & 6.35 & 2.41 & 6.00 & 1.71 & 3.87 \\
\hline
\end{tabular}

Table A.4 shows descriptive statistics for login times. Mean login times varied widely across conditions, ranging from 6.35 seconds in Chosen Text to 34.61 seconds in Object PassTiles. Login times were longest in the recognition condition, and longer in the cued-recall condition than in any of the recall conditions, seeming to indicate that login times increased with more recognition tasks.

As seen in Figure A.3, the distributions of login times were right skewed and leptokurtotic, making the use of parametric tests inappropriate.

A Kruskal-Wallis test showed significant differences in login times $\left(\chi^{2}(4)=51.31, p<\right.$ 0.001 ) between the different study conditions. Post-hoc pairwise Wilcoxon tests using a Bonferroni adjustment (Table A.5) showed that it took participants significantly less 


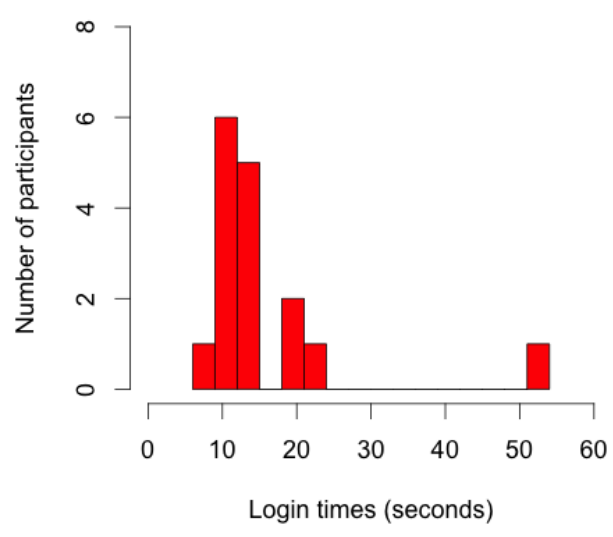

(a) BPT

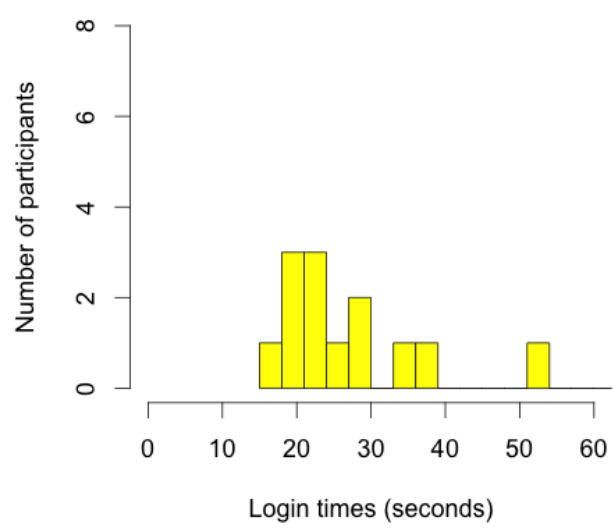

(c) OPT

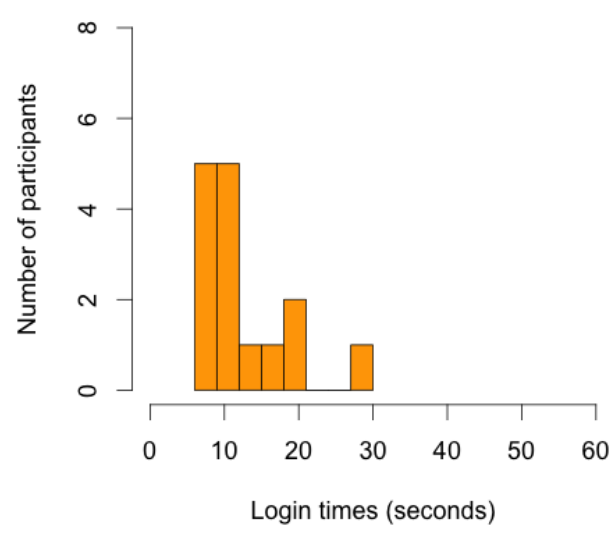

(b) IPT

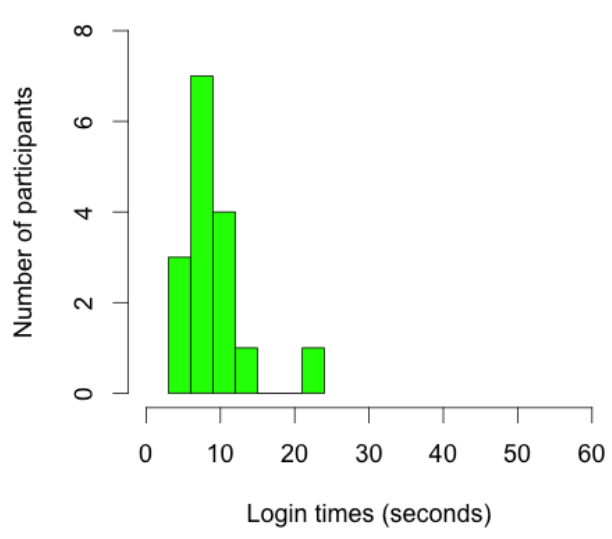

(d) AST

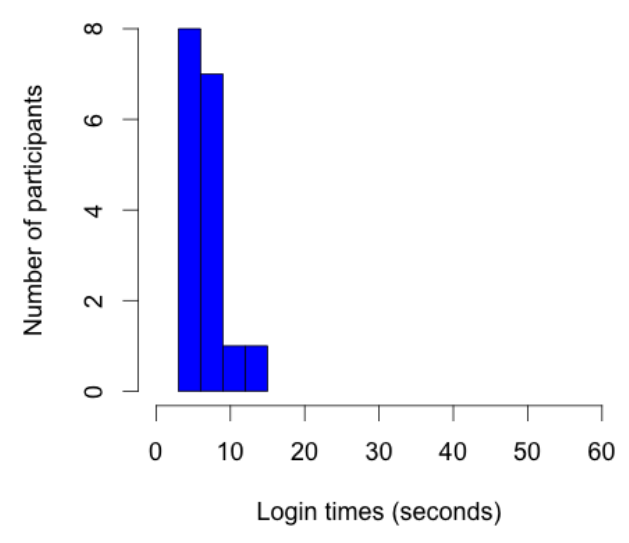

(e) $\mathrm{CHT}$

Figure A.3: Distributions of login time (in seconds) by number of participants for each study condition (Study 1). 
Table A.5: Pairwise Wilcoxon tests of login times using Bonferroni adjustment (Study $1)$.

\begin{tabular}{|l|r|r|}
\hline & $\mathrm{U}$ & $p$ \\
\hline BPT vs. IPT & 153.00 & 1.000 \\
BPT vs. OPT & 22.00 & 0.001 \\
BPT vs. AST & 213.00 & 0.014 \\
BPT vs. CHT & 260.00 & $<0.001$ \\
IPT vs. OPT & 13.00 & $<0.001$ \\
IPT vs. AST & 169.50 & 0.527 \\
IPT vs. CHT & 230.50 & 0.001 \\
OPT vs. AST & 236.00 & $<0.001$ \\
OPT vs. CHT & 255.00 & $<0.001$ \\
AST vs. CHT & 209.50 & 0.085 \\
\hline
\end{tabular}

time to log in using text passwords, and significantly longer to log in using Object PassTiles.

\section{Hypothesis Testing Summary}

The results of Study 1 showed that login times were significantly longer in the recognition condition (Object PassTiles). However, we found no significant differences in memorability between the different password systems. We were surprised by the lack of differences, and address this issue below.

\section{Security Analysis}

In all conditions but Chosen Text, passwords were randomly assigned, making the effective password space equal to the theoretical password space and pre-determining the security of the password system against guessing attacks. However, the password security in Chosen Text was largely determined by participants' choice of passwords.

When creating their passwords, participants were limited to 4 characters but were allowed to use any character set. In order to examine the security of the passwords chosen by participants in this condition, we looked at the incidence of password reuse, and at the use of patterns (e.g., dictionary words) in password selection.

If a participant asked whether they could reuse passwords, they were told that it 
would be more secure if they did not repeat their passwords, but the system did not enforce this policy. Of the 17 participants in Chosen Text, $59 \%$ had only one unique password, meaning that they reused the same password across all three of their accounts. Reusing passwords across different accounts is a security risk because it creates a single point of failure across multiple accounts and can potentially expose users' passwords to attackers.

The presence of dictionary words and common substitutions further narrows the space. Using the free dictionaries from the John the Ripper password cracking program [43], we were able to guess 8 passwords using a 9 bit dictionary. A further 4 passwords were able to be guessed using an 11 bit dictionary (that included the smaller 9 bit dictionary). A dictionary of digit combinations was able to guess 18 passwords that included only digits. In total, the John the Ripper dictionaries were able to guess $59 \%$ of the created passwords using a dictionary with a size of 13.5 bits.

The effective password space for the chosen text passwords was therefore considerably smaller than the password space for the assigned passwords used in the study. Because of this, it is not reasonable to directly compare the memorability or usability of the systems. In Chosen Text, the quantity of information that users are asked to use and remember is far less than in the assigned password conditions. In addition, users are given the opportunity to choose less secure, but more memorable passwords, which is not a choice given to users in the assigned password conditions. Because of the differing security levels, we feel that it is inappropriate to compare the memorability and usability of Chosen Text to the assigned password conditions.

\section{Password Recording}

We were surprised by the results we found for memorability. We had expected to see differences between conditions, and had not expected that most participants would be able to remember their passwords for the duration of the study. Although participants were instructed not to write their passwords down, and were restricted from doing so at the time of password creation in the lab, we had no means of controlling their behaviour once they left the lab. The post-test questionnaire asked participants whether they had written down any of their passwords. Since participants were aware that they were not supposed to write down their passwords, it seems likely 


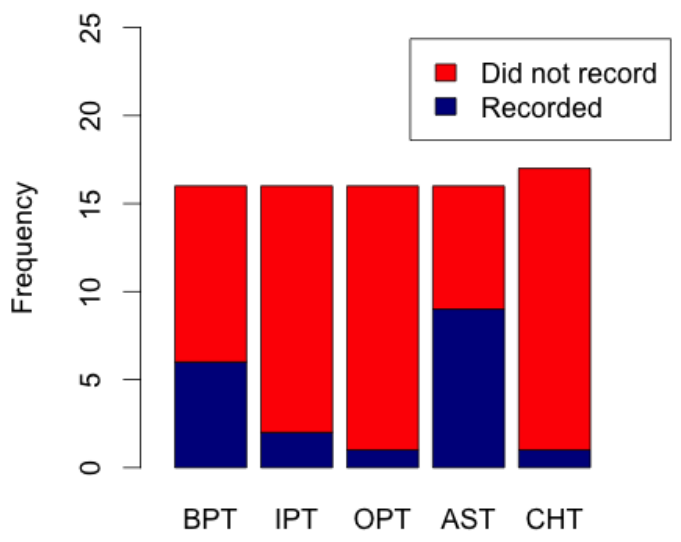

(a) Study 1

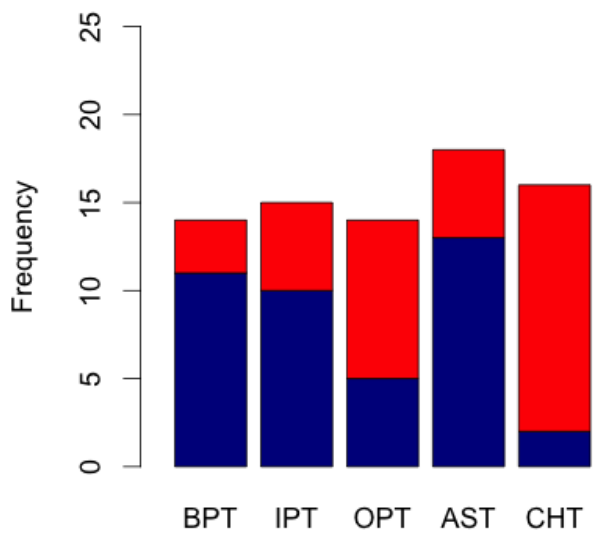

(b) Study 2

Figure A.4: Stacked bar plots showing frequency of reported password recording.

that our statistics on how many participants recorded their passwords represents a lower bound.

The number of participants who reported recording their passwords in each condition ranged from nine people in Assigned Text to just one person in Chosen Text and Object PassTiles. Figure A.4a shows a stacked barplot of the counts of password recording (and non-recording) in each condition. The corresponding percentages are: Blank PassTiles $=38 \%$, Image PassTiles $=13 \%$, Object PassTiles $=6 \%$, Assigned Text $=56 \%$, and Chosen Text $=6 \%$.

We used a chi-squared test to look for differences between the four assigned password conditions. A significant difference $\left(\chi^{2}(4)=17.97, p=0.001\right)$ in the number of password recordings was seen, and post-hoc pairwise chi-squared tests using a Bonferroni adjustment showed that the only significant difference in instances of password recording was between Assigned Text and Object PassTiles $\left(\chi^{2}(1)=7.13, p=0.046\right)$.

The proportion of participants who reported that they had written down their passwords in the free recall conditions was dramatic and likely had a strong effect on the memory time variable, since writing passwords down could allow participants to artificially appear to remember their passwords longer. This result sheds doubt on the hypothesis testing presented above. However, the differences in reported password 
recording across conditions may suggest that remembering recall-based passwords was more difficult for participants.

Our exploration of password recording in Study 1 was done post-hoc, without any hypothesis. However, the results of the analysis convinced us that this issue was worth exploring more explicitly. In later studies, we considered the issue a priori, and hypothesized that stronger password cues would lead to fewer reported password recordings.

\section{A.2.2 Study 2}

The goal of Study 2 was to replicate Study 1 with a larger sample size and more diverse participants. We conducted Study 2 using Crowdflower [38] as an outsourcing service for Amazon's Mechanical Turk [4] (MTurk). MTurk has been posited as an easily available source of participants for usable security experiments [78, 76], but little work has investigated the comparability of results obtained on MTurk to those obtained through more traditional means. We replicated almost all the details of our in-person study in our MTurk study. Only a few changes to the procedure were necessary. MTurk participants received training on the websites through an instructional webpage, instead of an in-person explanation. Also, instead of paying participants for the entire study at completion, we paid participants for each session as it was completed.

Study 2 had 77 participants and the demographics were similar to the main study in terms of age and expertise. However, the gender balance was approximately reversed (more men in the MTurk study) and fewer students participated in the MTurk study. Participants came from around the world, but the largest densities of participants were in the United States (27 participants) and India (26 participants).

\section{Hypothesis 1: Memory Time}

The distributions of memory time in Study 2 (Figure A.5) were similar to those in Study 1. However, memory times were slightly longer overall in Study 2 (Table A.6), probably because participants were free to complete the final task at any time after 


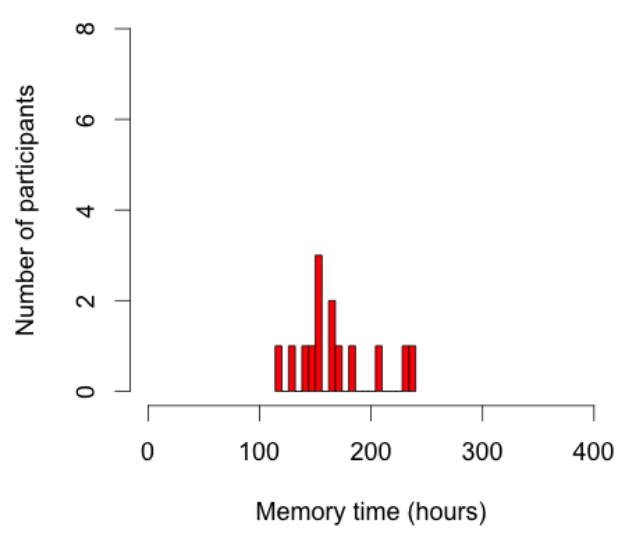

(a) $\mathrm{BPT}$

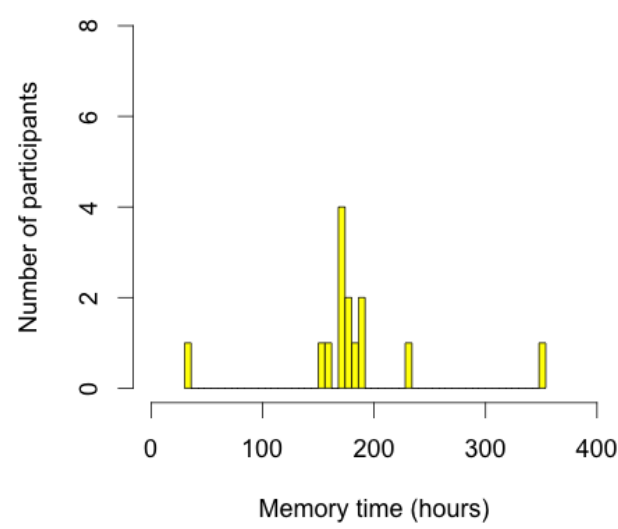

(c) OPT

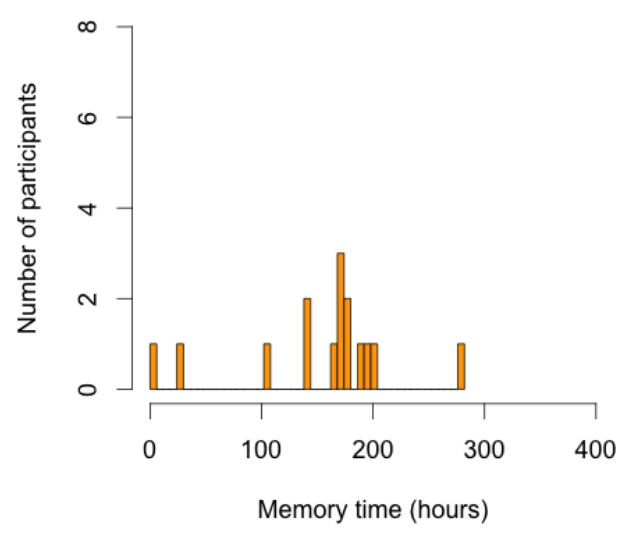

(b) IPT

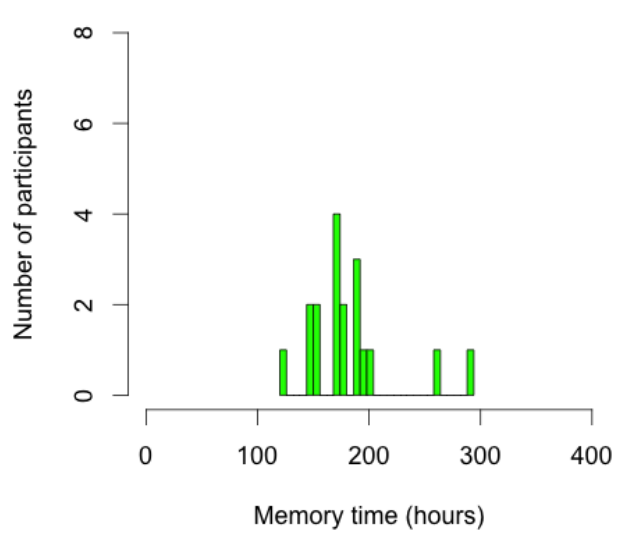

(d) AST

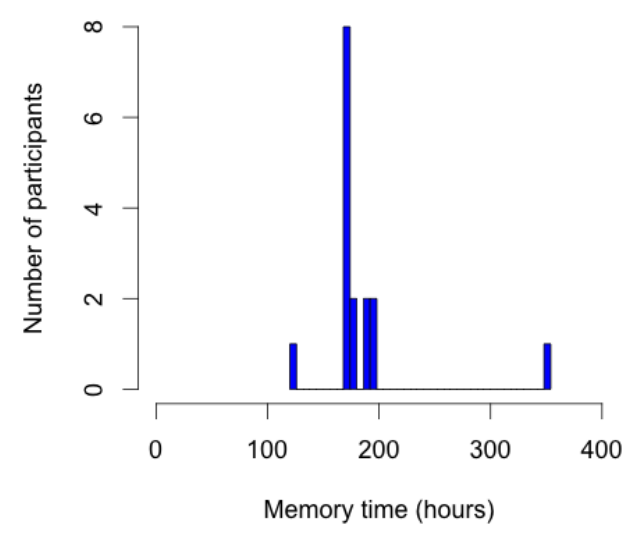

(e) $\mathrm{CHT}$

Figure A.5: Distributions of memory time (in hours) by number of participants for each study condition (Study 2). 
Table A.6: Memory time statistics (hours) (Study 2).

\begin{tabular}{|l|r|r|r|r|r|}
\hline & Mean & SD & Median & Skewness & Kurtosis \\
\hline BPT & 167.38 & 36.19 & 157.84 & 0.88 & 0.10 \\
IPT & 153.97 & 67.95 & 171.28 & -0.90 & 1.80 \\
OPT & 180.63 & 65.39 & 175.46 & 0.72 & 5.19 \\
AST & 181.76 & 39.48 & 172.70 & 1.50 & 2.87 \\
CHT & 184.37 & 47.83 & 171.21 & 3.11 & 11.80 \\
\hline
\end{tabular}

Table A.7: $t$-tests of memory time (Study 2).

\begin{tabular}{|l|r|r|r|}
\hline & $t$ & df & $p$ \\
\hline AST vs. BPT & 1.07 & 29 & 0.854 \\
AST vs. IPT & 1.40 & 22 & 0.912 \\
AST vs. OPT & 0.06 & 20 & 0.522 \\
\hline
\end{tabular}

the final email arrived. As in Study 1, memory times were similar throughout the conditions, and there were no significant differences in memory time between Assigned Text and each of the graphical password conditions (Table A.7), or among the three graphical password conditions $(F(2,40)=0.75, p=0.479)$.

\section{Hypothesis 2: Resets}

Table A.8: Password reset statistics (Study 2).

\begin{tabular}{|l|r|r|r|r|r|}
\hline & Mean & SD & Median & Skewness & Kurtosis \\
\hline BPT & 1.36 & 1.45 & 1 & 0.15 & -2.15 \\
IPT & 2.00 & 3.42 & 0 & 2.10 & 4.63 \\
OPT & 0.86 & 1.96 & 0 & 2.82 & 8.30 \\
AST & 0.56 & 1.04 & 0 & 1.77 & 1.88 \\
CHT & 0.19 & 0.75 & 0 & 4.00 & 16.00 \\
\hline
\end{tabular}

The distributions of password resets in Study 2 (Figure A.6) were again similar to those in Study 1. Participants in Study 2 reset their passwords more often than those in Study 1, particularly in the three graphical password conditions (Table A.8). This was possibly because they received less mandatory training in the use of the password systems, and may have experienced more trouble in remembering their passwords, or needed more attempts to understand the functioning of the password system.

One difference was that Study 2 had more password resets in Object PassTiles, and this affected the differences between conditions. In Study 2, there were no significant 


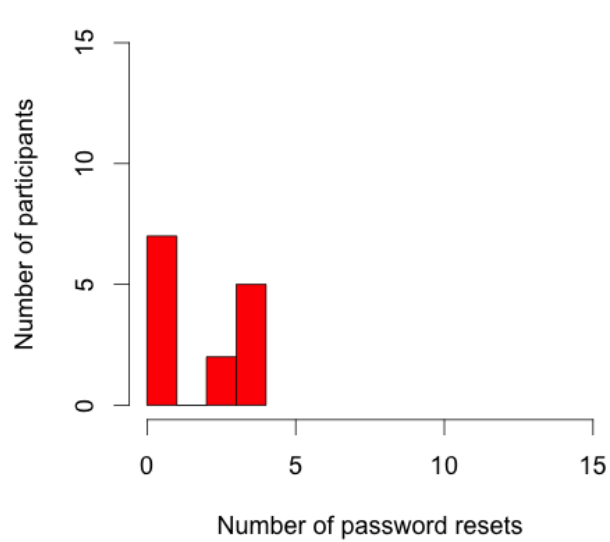

(a) BPT

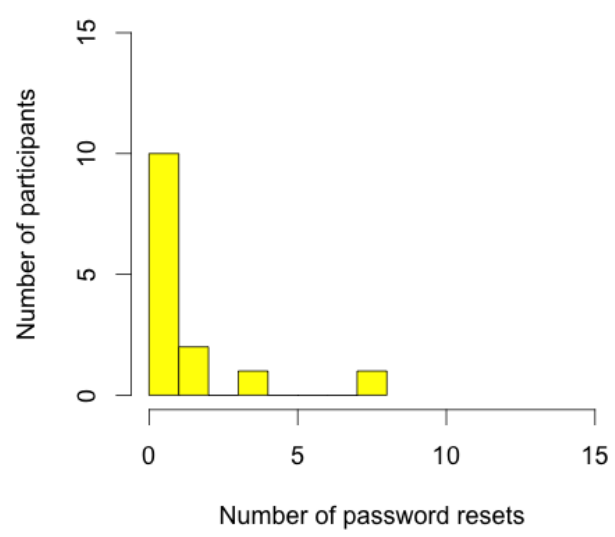

(c) OPT

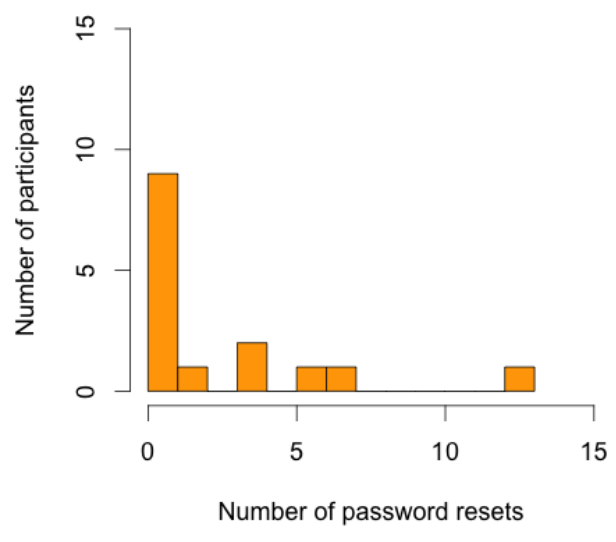

(b) IPT

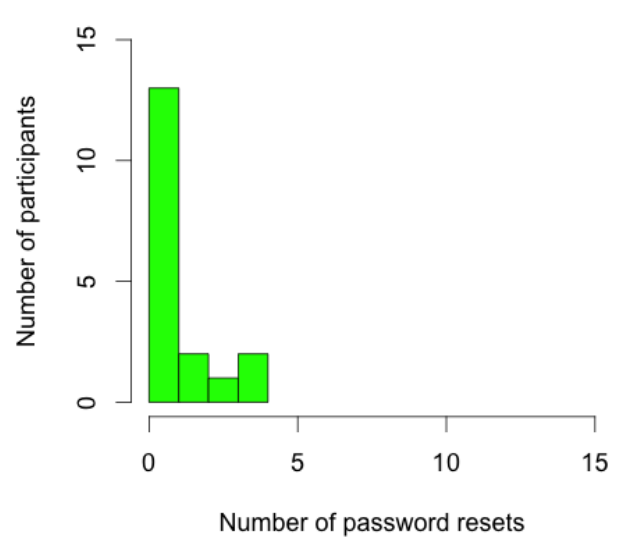

(d) AST

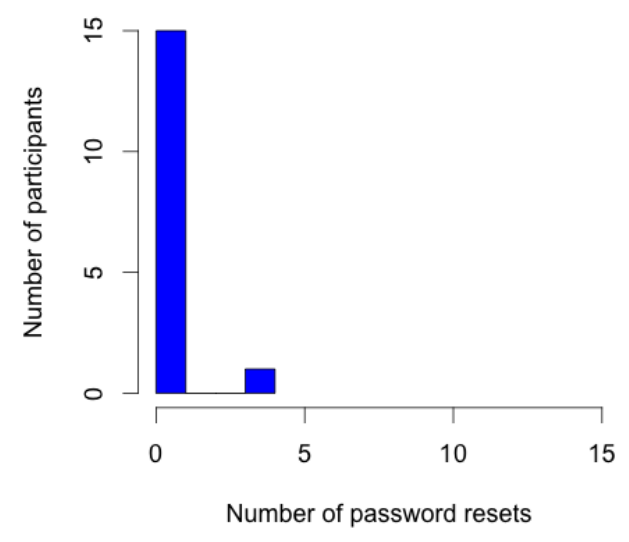

(e) $\mathrm{CHT}$

Figure A.6: Distributions of password resets by number of participants for each study condition (Study 2). 
Table A.9: Wilcoxon tests of password resets comparing the assigned text condition with each of the graphical password conditions (Study 2).

\begin{tabular}{|l|r|r|}
\hline & $U$ & $p$ \\
\hline AST vs. BPT & 90.50 & 0.943 \\
AST vs. IPT & 109.50 & 0.869 \\
AST vs. OPT & 124.00 & 0.548 \\
\hline
\end{tabular}

differences in password resets between Assigned Text and any of the graphical password conditions (Table A.9). As in Study 1, we saw no significant differences in password resets among the three graphical password conditions $\left(\chi^{2}(2)=1.40, p=0.497\right)$, meaning that the increased resets in Object PassTiles were not statistically significant. When we compared the number of resets in Chosen Text with each of the assigned password conditions, we found that there were significantly fewer resets in Chosen Text than in Blank PassTiles $(U=64.00, p=0.005)$ or Image PassTiles ( $U=78.50, p=0.013$ ), but no significant difference between Chosen Text and Object PassTiles or Assigned Text.

\section{Hypothesis 3: Login Times}

Table A.10: Login time statistics (seconds) (Study 2).

\begin{tabular}{|l|r|r|r|r|r|}
\hline & Mean & SD & Median & Skewness & Kurtosis \\
\hline BPT & 21.87 & 11.41 & 19.17 & 1.27 & 0.93 \\
IPT & 19.95 & 13.87 & 14.85 & 2.80 & 9.14 \\
OPT & 30.23 & 9.45 & 31.08 & 0.82 & 1.30 \\
AST & 10.72 & 5.33 & 9.54 & 1.36 & 2.86 \\
CHT & 5.16 & 1.79 & 4.71 & 1.97 & 5.58 \\
\hline
\end{tabular}

Median login times followed the same pattern (Figure A.7), but were slightly longer overall in Study 2 than in Study 1 (Table A.10). This is probably due to participants with slower computers, and the network delays associated with participants in diverse geographic regions. As in Study 1, a Kruskal-Wallis test showed significant differences in login time among the study conditions $\left(\chi^{2}(4)=52.99, p<0.001\right)$. Post-hoc pairwise Wilcoxon tests showed significant differences in all pairwise comparisons except between Image PassTiles and Blank PassTiles, and between Object PassTiles and Blank PassTiles (Table A.11). This was slightly different from the first study, and the difference comes from the longer and more similar login times for the graphical password conditions were longer and more similar in Study 2 than in Study 1. 


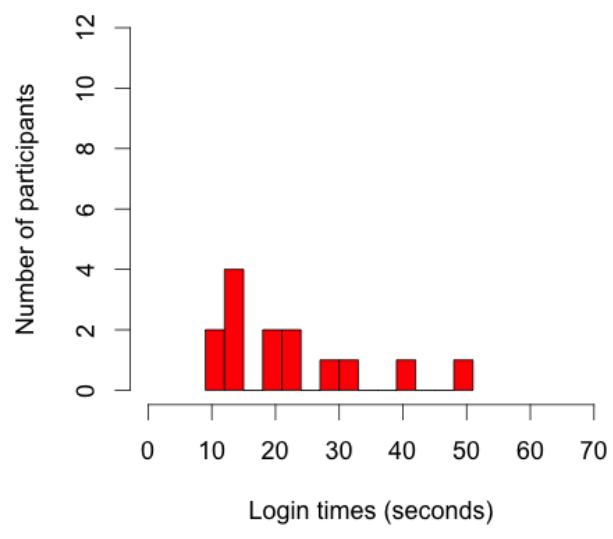

(a) BPT

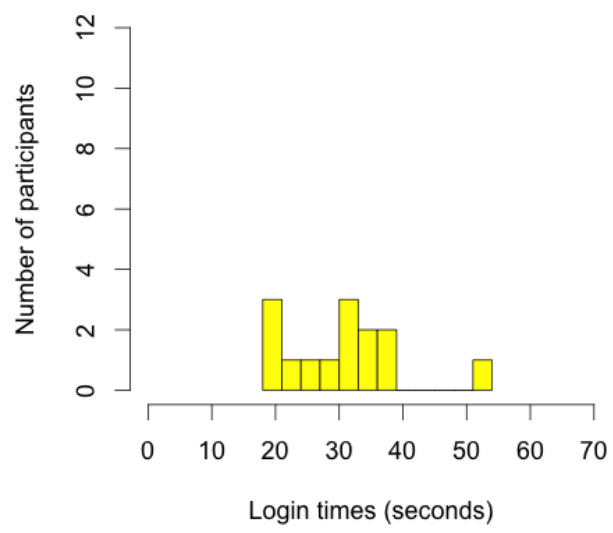

(c) OPT

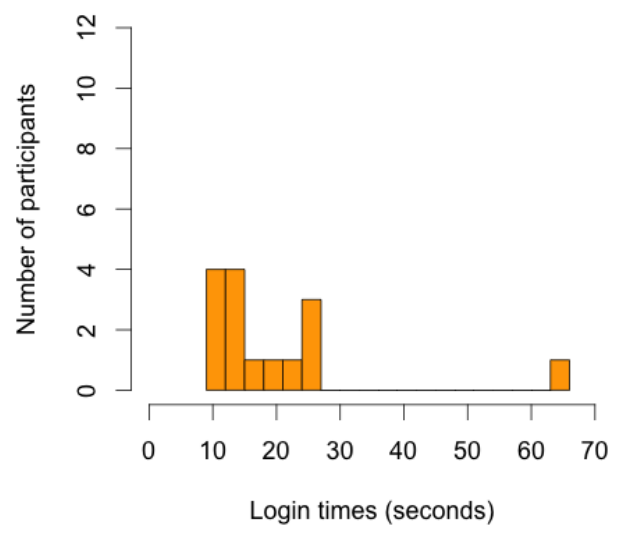

(b) IPT

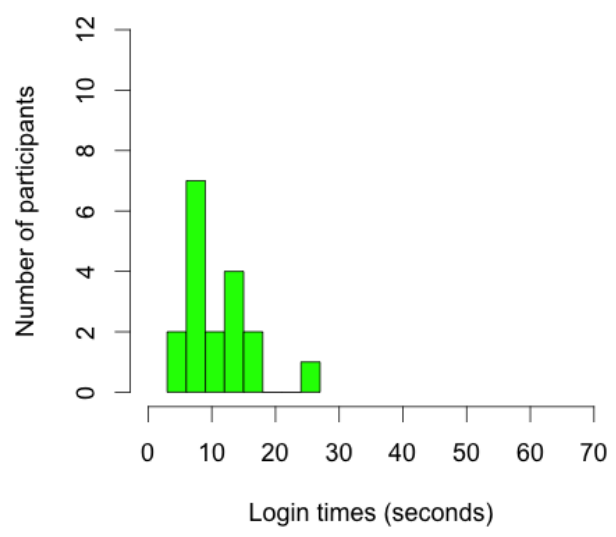

(d) AST

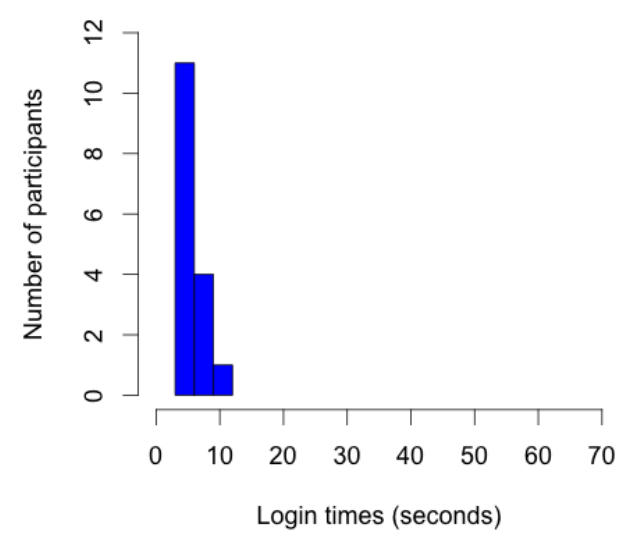

(e) $\mathrm{CHT}$

Figure A.7: Distributions of login times by number of participants for each study condition (Study 2). 
Table A.11: Pairwise Wilcoxon tests of login times using Bonferroni adjustment (Study 2).

\begin{tabular}{lrr}
\hline & $\mathrm{U}$ & $p$ \\
\hline BPT vs. IPT & 122.50 & 1.000 \\
BPT vs. OPT & 50.00 & 0.274 \\
BPT vs. AST & 217.50 & 0.005 \\
BPT vs. CHT & 224.00 & $<0.001$ \\
IPT vs. OPT & 34.00 & 0.013 \\
IPT vs. AST & 214.50 & 0.043 \\
IPT vs. CHT & 238.00 & $<0.001$ \\
OPT vs. AST & 247.00 & $<0.001$ \\
OPT vs. CHT & 224.00 & $<0.001$ \\
AST vs. CHT & 257.50 & 0.001 \\
\hline
\end{tabular}

\section{Password Recording}

As in Study 1, participants were asked in the post-test questionnaire whether they had written down any of their passwords. Figure 4.5b shows the participants in each condition in Study 2 who reported writing down their passwords. More participants reported recording their passwords in Study 2 than in Study 1. In Study 2, participants were not instructed not to write their passwords down, partly for ecological validity, and partly to avoid false responses on the post-test questionnaire. Again, a chi-squared test showed a significant difference in the number of password recordings $\left(\chi^{2}(4)=19.69, p<0.001\right)$ between the four assigned password conditions. In our added hypothesis, we speculated that we might again see the relationship between the strength of cues and password recording. In Study 2, the ordering of password write-down frequency was the same as in Study 1, which would be an extremely rare event to occur by chance. There are 5 conditions and thus $5 !=120$ possible orderings, giving a probability of $<0.01$ of seeing the Study 1 pattern repeated in Study 2. This strengthens our suggestion that the incidence of password recording reflects the difficulty of memorability across the conditions. 


\section{$\underline{\text { Appendix B }}$ \\ Password Life Cycle Questionnaire Script}

This questionnaire asks questions about your demographics and your password habits. The survey is anonymous and confidential, and all questions are optional.

The second part of the survey asks about your password habits. Please keep your own passwords and habits in mind as you complete the questions, but NEVER share your own passwords.

\section{B.1 Demographics}

1. What is your gender?

2. How old are you?

3. What is your occupation?

4. Are you a student?

5. (If a student) What degree program are you registered in?

6. What is your first language? 


\section{B.2 Passwords}

\section{B.2.1 Existing Passwords}

In this section we're going to get you to think about the passwords and accounts you have in your real life. Some of these questions will be difficult to answer accurately please give it your best estimate, and explain to us your reasoning.

1. How many accounts do you have in total (estimate)?
(a) Emails?
(b) Banks?
(c) Forums?
(d) Travel (airlines, hotels, trains, reviews)?
(e) Social networks?
(f) School?
(g) Newspapers/online media?
(h) Movies/music/entertainment?
(i) Utilities/services?
(j) Online shopping?
(k) Gaming?
(l) Pictures?

(m) Cloud storage?

(n) Other?

2. How many accounts do you use in an average week (estimate)?
(a) In the daytime (at school/work)?
(b) In the evening (at home)?
(c) On the weekend? 
3. How many unique passwords do you have?

4. Do you ever reuse passwords?

5. How often do you reuse passwords? (never, rarely, sometimes, frequently, always)

6. Do you reuse multiple passwords, or only one?

7. How many passwords do you reuse?

8. What kind of devices do you ever enter passwords on? (smartphone, tablet, laptop, desktop)

9. Do you ever consider the devices you will be using to enter passwords when you create your passwords? What kind of considerations do you make?

10. Do you ever enter passwords on computers that do not belong to you (for example, at the library, at work, at a friend's house, at an internet cafe)

11. Do you ever save your passwords in a computer program?

12. What programs do you currently use to save passwords? (List all)

(a) Firefox

(b) Internet Explorer

(c) Safari

(d) Chrome

(e) iCloud/Apple Keychain

(f) LastPass

(g) 1password

(h) KeePass

(i) Roboform

(j) Other?

13. Do you ever click the "remember me" box on websites?

14. Do you ever write your passwords down? 
15. How do you store or refer to written down passwords?

\section{B.2.2 Setting up a New Account}

Often, we end up having to create a new account so that we can read an article, or check out in an online store. Imagine you're creating a new account that you don't expect to use very often when you answer the next set of questions.

1. How would you go about choosing a password for the new account?

2. Would you consider reusing a password?

3. If reusing a password, how would you decide which password to reuse?

4. If you decide to reuse a password, what do you do if the site tells you it isn't allowed?

5. How will you keep track of which password goes with the new account?

\section{B.2.3 Changing a Password}

Sometimes, we have to change the password for an existing account. The next set of questions is about password changes and resets.

1. Do you ever change your password when the site doesn't insist on it?

2. How often do you change your passwords? (never, rarely, sometimes, frequently, often)

3. What is the most common reason you change your password?

4. Do you ever reset your password because you don't remember the password?

5. How often do you do this?

6. If you change your password, how do you pick the new password? 


\section{$\underline{\text { Appendix C }}$}

\section{Life Cycle Survey Questionnaire}

All questions on the survey are optional. This survey is divided into 6 sections, each focusing on a different aspect of password management:

\section{C.1 Password Use}

1. For each of the following categories, how many accounts do you have? Please include all accounts that you have, even if you don't use them often.
(a) Email
(b) Banking
(c) Forums/message boards/chatrooms
(d) Travel (airlines, hotels, trains, reviews)
(e) Social networks
(f) School/work
(g) Newspapers/online media
(h) Movies/music/entertainment
(i) Utilities/services 
(j) Online shopping

(k) Gaming

(l) Pictures

(m) Cloud storage

(n) Other

2. In an average week, how many accounts do you use:

(a) In the daytime (at school, or at work)?

(b) In the evening?

(c) On the weekend?

3. How many unique passwords do you have?

4. Do you reuse passwords? (Y/N)

5. If so, how many passwords do you reuse?

6. Do you save your passwords in a web browser (for example, Internet Explorer, Firefox, Safari, or Chrome)? (Y/N)

7. If so, which browser(s) do you use to save passwords?
(a) Internet Explorer
(b) Mozilla Firefox
(c) Chrome
(d) Safari
(e) Opera
(f) Other

8. Do you use a password manager program? $(\mathrm{Y} / \mathrm{N})$

9. If so, which program?
(a) LastPass
(b) KeePassX 
(c) Dashlane

(d) Roboform

(e) 1Password

(f) Password Genie

(g) Other

10. Do you ever click the checkbox to set a cookie to keep you logged into websites (often reads "remember me" or "keep me logged in")? ( $\mathrm{Y} / \mathrm{N}$ )

11. Do you write any of your passwords down (either on the computer or on paper)? $(\mathrm{Y} / \mathrm{N})$

12. How do you store your written down passwords (for example, on a post-it note, or in a spreadsheet)?

13. How do you make sure your passwords are available when you need them?

14. Do you use single sign-on or federated identity services (for example, logging in using your Facebook or Google credentials) to log into other accounts? (Y/N)

15. If so, which service do you use?
(a) Google
(b) Facebook
(c) OpenID
(d) Twitter
(e) Other

\section{C.2 Choosing Passwords}

On a scale from 1 to 5 , where 1 is never and 5 is always, please rate how frequently you do the following:

1. When creating passwords, I include: 
(a) My own birthday

(b) The birthday of a loved one

(c) My own name

(d) The name of a loved one

(e) Phone numbers (or parts of phone numbers)

(f) Addresses (or parts of addresses)

(g) Information about the website where the password will be used

(h) Information about the specific item I am purchasing

(i) Part of another password

2. When creating passwords, I include information relating to:
(a) Hobbies
(b) Movies
(c) Music/song lyrics
(d) Sports
(e) Religious beliefs
(f) Personal goals
(g) Pets

3. I use the same symbol(s) in my passwords.

4. I capitalize the same letter(s) in my passwords.

5. I use the same digit(s) in my passwords.

6. I have a systematic way of combining information to create new passwords. $(\mathrm{Y} / \mathrm{N})$

7. My system includes (check all that apply):

(a) A piece of information about the website

(b) A piece of personal information 
(c) A standard digit

(d) A standard symbol

(e) A standard word

(f) Other

8. My passwords correspond to a particular time period in my life. $(\mathrm{Y} / \mathrm{N})$

9. My passwords correspond to a particular username. $(\mathrm{Y} / \mathrm{N})$

10. My passwords correspond to a particular password policy. (Y/N)

11. When the password policy requires me to add a digit, symbol, or capital letter, I:

(a) I add a particular symbol in my passwords.

(b) I add a particular digit in my passwords.

(c) I capitalize a particular letter in my passwords.

\section{C.3 Password Reuse}

On a scale from 1 to 5 , where 1 is never and 5 is always, please rate how frequently you do the following:

1. I reuse passwords. $(\mathrm{Y} / \mathrm{N})$

2. I reuse passwords because:
(a) It is fast
(b) It is secure
(c) It is easy-to-remember
(d) It is convenient

3. I reuse the same password on:

(a) Accounts that I consider to have similar security needs. 
(b) Accounts that have similar content (for example, email accounts).

(c) Accounts that I access for work or school.

(d) Accounts that I do not access very often.

(e) Accounts that I access a lot.

(f) Accounts that have the same password policy rules.

(g) Accounts that I created around the same time.

4. I do not reuse passwords on:

(a) Online banking websites

(b) Websites that store credit card information

(c) Websites that collect credit card information (without storing it)

(d) Accounts with my name

(e) Accounts with my home address

(f) Accounts with my workplace

(g) Accounts with my phone number

(h) Accounts with my birthday

(i) Accounts that I share with my partner or spouse

(j) Accounts that I share with my children

(k) Accounts that I share with my friends

(l) Accounts that I share with my co-workers

5. I have a "primary" or "go-to" password that I reuse. $(\mathrm{Y} / \mathrm{N})$

6. I create variations of my "go-to" password to use on different accounts. (Y/N)

7. I have a standard way of creating variations of my "go-to" password. (Y/N) 


\section{C.4 Saving Passwords}

On a scale from 1 to 5 , where 1 is never and 5 is always, please rate how frequently you do the following:

1. I write my passwords down.

2. I write my passwords down on paper (for example, on a post-it note, or in a notebook). (Y/N)

3. I write my passwords down electronically (for example, on the computer, or on a mobile device). $(\mathrm{Y} / \mathrm{N})$

4. I store my passwords electronically by:

(a) I email my passwords to myself.

(b) I save my passwords in the cloud (for example, in Dropbox, iCloud, or Google Drive).

(c) I save my passwords in a spreadsheet

(d) I save my passwords in a word document

5. I password protect the file where I save my passwords.

6. I write my passwords down in case I need them.

7. I do not usually refer to my written down passwords.

8. I write my passwords down so that someone else can access them.

9. I write my passwords down temporarily while I am memorizing them.

10. I write down high-security passwords.

11. I write down low-security passwords.

12. I write down frequently-used passwords.

13. I write down infrequently-used passwords.

14. I write down passwords that cannot be reset.

15. I write down assigned passwords. 
16. I write down hints for my passwords.

17. I write down parts of my passwords.

18. I write down usernames.

19. I save my passwords in a web browser. ( $\mathrm{Y} / \mathrm{N})$

20. I save my passwords in a web browser in a specific context:
(a) At work
(b) At home
(c) On my mobile device

21. I do not save these passwords in a web browser:
(a) Banking passwords
(b) Email passwords
(c) Social networking passwords
(d) Online shopping passwords where credit card information is collected
(e) Online shopping passwords where credit card information is not collected
(f) Other

22. I save my passwords in a dedicated password manager. $(\mathrm{Y} / \mathrm{N})$

23. I use a password manager to generate passwords.

24. I save high-importance accounts in a password manager.

25. I save low-importance accounts in a password manager.

26. I use a password manager because:
(a) It is more secure
(b) It is more convenient
(c) It is easy-to-use

27. I do not use a password manager because:

(a) I do not trust them. 
(b) I do not have a problem remembering my passwords.

(c) I do not know which one to use.

(d) I do not want to set one up.

(e) I do not know what a password manager is.

(f) Other

\section{C.5 Password Changes}

On a scale from 1 to 5 , where 1 is never and 5 is always, please rate how frequently you do the following:

1. I change my passwords regularly. $(\mathrm{Y} / \mathrm{N})$

2. I have changed my passwords in response to particular event. (Y/N)

3. What kind of event was this?

(a) Media coverage of a security breach

(b) Advice articles about good security practices

(c) Advice from a friend or family member

(d) Advice from an IT expert

(e) A major life change or personal event (for example, the birth of a child, a move, or the end of a relationship)

(f) Sharing a password with another user

(g) Realizing that an account has been hacked (for example, realizing that an email address is sending spam)

(h) Other

4. When I am forced to change my passwords:

(a) I change to a variation of the previous password.

(b) I add new parts to a piece of another existing password. 
(c) I make slight modifications to another existing password.

(d) I choose an entirely new password.

5. When I forget my password, I:

(a) Use the "forgot password" button to reset the password.

(b) Refer to where I have written the password down.

(c) Rely on the verification questions.

(d) Guess at the password.

(e) Try to recreate the process I followed when creating the password.

(f) Look at the password creation policy.

6. On average, I forget my passwords:
(a) Once a day
(b) Once a week
(c) Once a month
(d) 3-4 times a year
(e) Once a year or less

7. When I forget my passwords and must reset them:
(a) I change the password to what I thought it should be.
(b) I reuse another existing password.
(c) I make slight modifications to another existing password.
(d) I choose an entirely new password.

\section{C.6 Password Security}

On a scale from 1 to 5 , where 1 is strongly disagree and 5 is strongly agree, please rate your agreement with the following: 
1. I think that I am doing a good job protecting my accounts.

2. I am aware that I could do more to protect my accounts.

3. I am doing my best to protect my accounts.

4. I do not have time to pay attention to security.

5. I do not feel that my accounts are likely to be attacked.

6. I do not know whom to trust for security advice.

7. I do not know where to look for help with passwords and security.

8. I have a standard person that I ask for help with computer security problems.

9. Keeping track of my passwords is difficult.

10. I find it easy to pick new passwords.

11. I have had an account hacked, or become aware of a security breach in my accounts.

12. I have changed my password management strategies over time.

13. I have reused passwords from high-security accounts on low-security accounts.

14. I have chosen new passwords for high-security accounts, and then reused the old password on other accounts.

15. I give some accounts more "security attention" than others.

16. It is hard to decide where to focus my "security attention".

17. My "security attention" is limited.

18. Some accounts deserve more "security attention" than others.

\section{C.7 Demographics}

1. My gender is: $(\mathrm{M} / \mathrm{F})$

2. My age is: 

(a) $18-25$
(b) 26-35
(c) $36-45$
(d) $46-55$
(e) $56-65$
(f) $66+$

3. What is your mother tongue/first language?

4. What is your highest level of completed education:
(a) High school
(b) Trade school or apprenticeship
(c) College or professional school
(d) Undergraduate (bachelors) degree
(e) Masters or PhD

5. What was/is your field of study?

6. What is your current occupation?

7. On a scale from 1 to 5 , where 1 is novice and 5 is expert, how knowledgeable are you about computers?

8. On a scale from 1 to 5 , where 1 is novice and 5 is expert, how knowledgeable are you about computer security? 